\title{
Concurrent Hydroclimatic Hazards from Catchment to Global Scales
}

\author{
by \\ Paolo De Luca
}

Doctoral Thesis

Submitted in partial fulfilment of the requirements for the award of Doctor of Philosophy (PhD) of Loughborough University

(August 2019)

C by Paolo De Luca (2019) 


\section{Abstract}

Interactions between multiple hazards can cause socio-economic damages that exceed those expected by the individual hazard components. Over the past decade, the multi-hazards paradigm has emerged to the extent that the Sendai Framework for Disaster Risk Reduction 2015-2030 advocated a multihazard approach. This thesis examines three types of concurrent hydroclimatic hazards that can occur at catchment to global scales.

The first multi-hazard is the link between multi-basin flooding (MBF) and extra-tropical cyclones (ETCs) over Great Britain during the period 1975-2014. Results show that during the most geographically widespread MBF episode, up to 108 river catchments (or $46 \%$ of the study area) recorded a peak flow annual maximum within a 16-day window. Most extreme MBF episodes were linked to cyclonic Lamb Weather Types (LWTs), atmospheric rivers and very severe gales. These episodes were associated with significant socio-economic impacts due to widespread flooding.

The second hazard was observed (1971-2000) and projected (2011-2100) LWTs, whose seasonal frequency and persistence are associated with multi-hazards over the British Isles (BI). Daily sea-levelpressure data from two reanalyses products, one subjective weather pattern catalogue and an ensemble of 10 Atmosphere-Ocean General Circulation Models (AOGCMs) were used to compute LWTs. Results showed that the AOGCMs are overall able to reproduce historical weather pattern persistence, which, along with annual frequency ( $\mathrm{p}$-value $<0.01$ ), is projected to significantly increase anticyclonic and decrease cyclonic LWTs, in summer and autumn respectively. This implies a higher risk of drought, heatwaves and air pollution events in summer but reduced likelihood of flooding and severe gales in autumn by the end of the 21 st century. By 2100 , the AOGCMs suggest a significant increased risk of concurrent flood-wind hazards during winter. In summer, the strength of the nocturnal Urban Heat Island (UHI) of London is expected to intensify by about $0.15{ }^{\circ} \mathrm{C}$ by the end of the century, contributing to higher chances of combined heatwave-air pollution events.

The third type of multi-hazard investigated was the spatio-temporal concurrence of global wet and dry hydrological extremes, during the 1950-2014 period. The analysis was conducted using the monthly self-calibrated Palmer Drought Severity Index based on the Penman-Monteith model (sc_PDSI_pm) - a global gridded dataset that has been applied in similar, but single-hazard, investigations. Results showed that the land area impacted by extreme dry and wet-dry events significantly increased over the 
observational period. The most geographically widespread wet-dry event covered a total area of 21 million $\mathrm{km}_{2}$ (or $14 \%$ of the global land area) with documented flood and drought impacts over diverse regions. Two new metrics were developed to provide more insight into the combined wet and dry hazards: the wet-dry (WD) ratio and the extreme transition (ET) time interval. The former quantifies the predominance of wet or dry extremes over a given area, whereas the ET measures the average separation time between the opposite extremes (i.e. between wet to dry or dry to wet transitions). The WD-ratio reveals a predominance of wet over dry extremes in the USA, northern and southern south America, northern Europe, north Africa, western China and most of Australia. The ET median for wet to dry is $\sim 27$ months, and 21 months for dry to wet. Global correlations between wet-dry hydrological extremes and El Niño Southern Oscillation (ENSO), Pacific Decadal Oscillation (PDO) and American Multidecadal Oscillation (AMO) were also investigated. ENSO and PDO showed similar correlation patterns, with the former significantly impacting a larger area. On the other hand, the AMO showed an almost inverse spatial correlation pattern, with an overall larger area impacted.

The findings presented in this thesis could be informative for emergency responders and relief agencies, disaster risk reduction practitioners, and (re)insurance companies. For instance, multi-basin flooding co-occurring with ETCs could overwhelm emergency response that depends on support from neighbouring regions that are similarly affected. Economic damages could exceed those insured by households and businesses. Projected rises in nocturnal UHI intensity in London could exacerbate heat-stress and, when combined with episodes of poor air quality, increase the likelihood of health problems amongst vulnerable groups. Furthermore, concurrent wet and dry hydrological extremes could be significant for organizations with global assets or sensitive supply chains, and the hydropower, agricultural and transport sectors more generally. Global maps generated of major wetdry events and the WD-ratio could also be integrated into a seasonal forecasting product, to help stakeholders in hedging the risk. Key opportunities for further research on multi-hazards are future hydroclimatic projections in the light of anthropogenic climate change and the application of new statistical techniques that could help in discerning the driving physical processes. 


\section{Table of Contents}

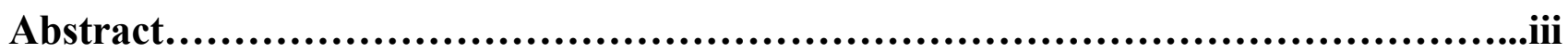

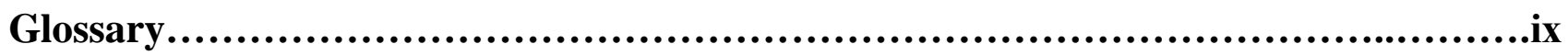

List of Figures..............................................................................ii

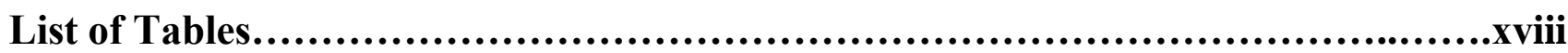

Chapter 1: Introduction.................................................................21

Chapter 2: Literature review.........................................................29

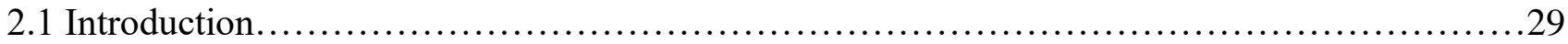

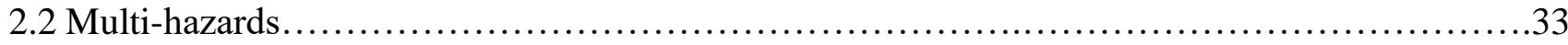

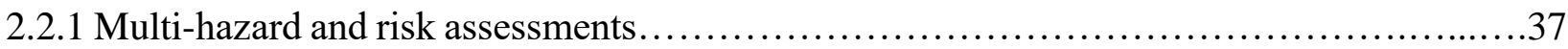

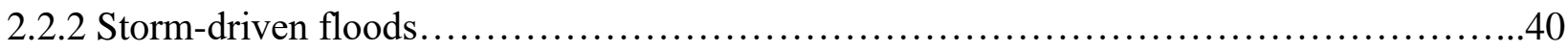

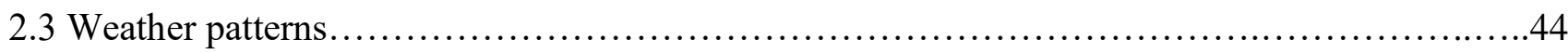

2.4 Hydrological extremes and modes of climate variability .....................................50

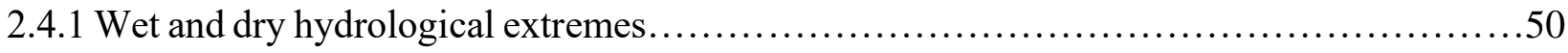

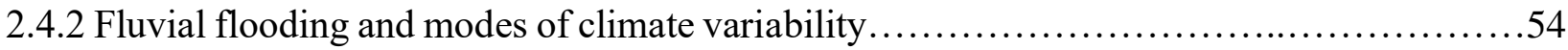

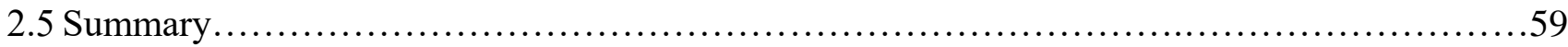

Chapter 3: Extreme multi-basin flooding linked with extra-tropical cyclones.............61

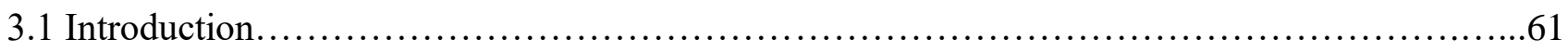

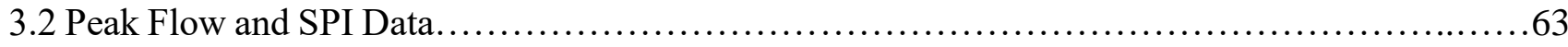

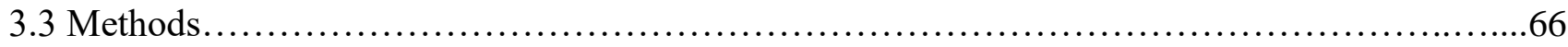

3.3.1 Quantifying multi-basin flooding episodes............................................66

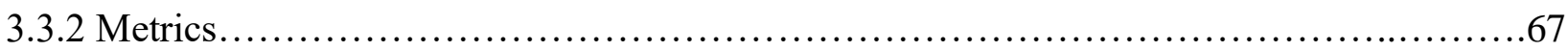

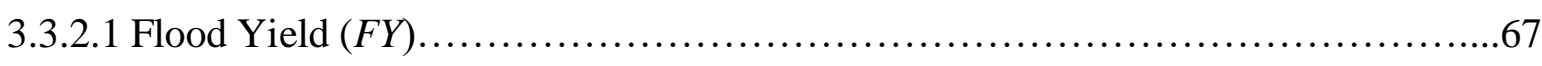

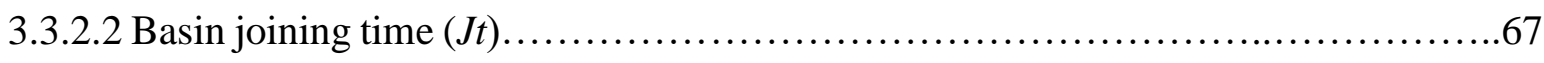

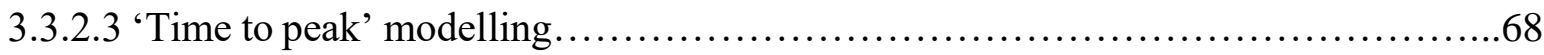

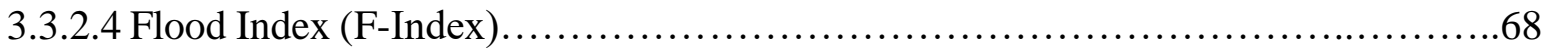

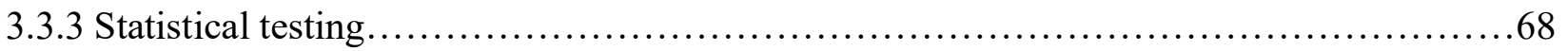


3.3.3.1 Multi-basin episodes.....................................................68

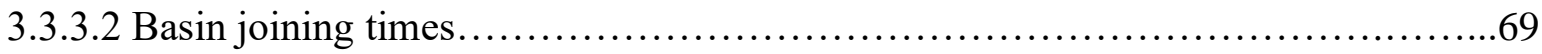

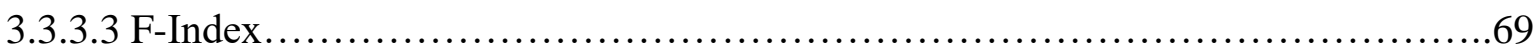

3.3.3.4 Multi-basin flooding and Atmospheric Rivers..............................69

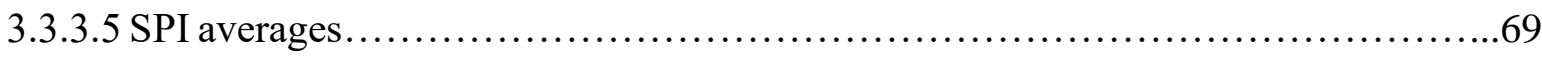

3.3.3.6 Peak flows and very severe gales......................................... 70

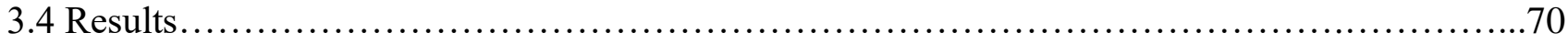

3.4.1 Characterizing severe MBF episodes.............................................. 70

3.4.2 Relationship to inundation episodes........................................... 77

3.4.3 Relationship to atmospheric patterns........................................ 78

3.4.4 Relationship with antecedent soil moisture conditions..............................80

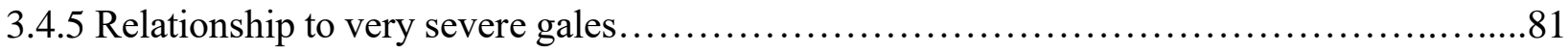

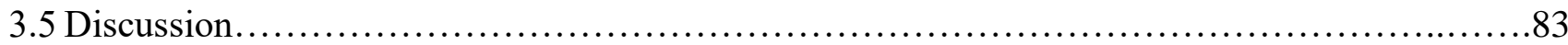

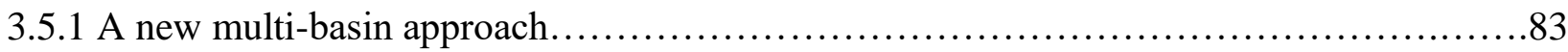

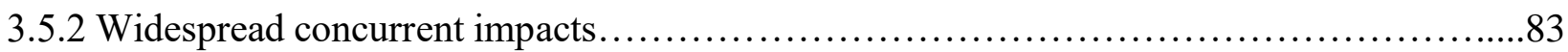

3.5.3 Compound flood and wind impacts ................................................ 85

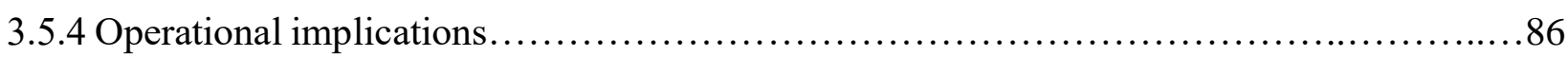

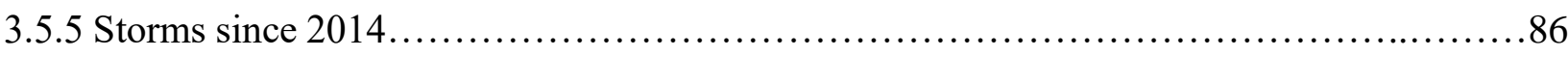

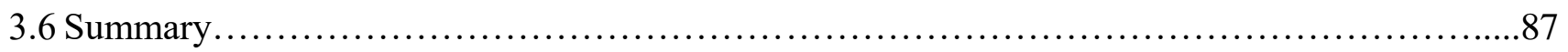

Chapter 4: Past and projected weather pattern persistence associated with multi-hazards in the British Isles........................................................................89

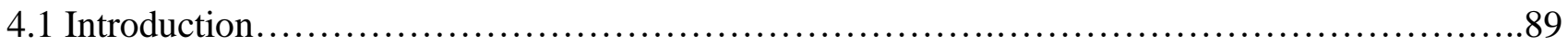

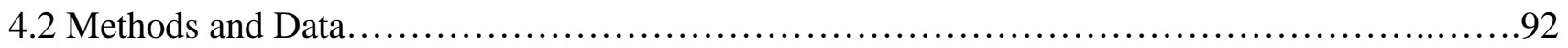

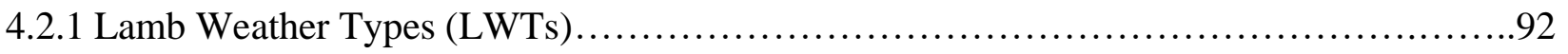

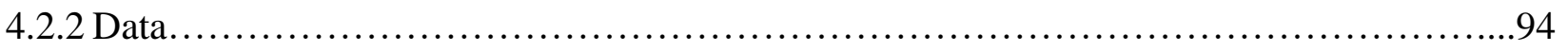

4.2.3 Persistence and trend analyses..............................................96

4.2.4 Indices of winter flood-wind hazards and summer UHI intensity .....................97

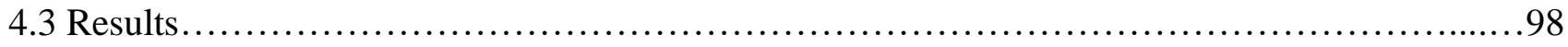

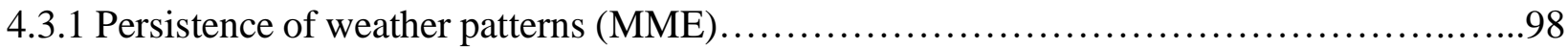

4.3.2 Persistence of weather patterns (by model) ...................................... 102

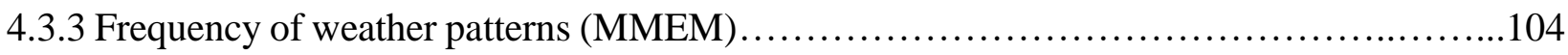

4.3.4 Application to future multi-hazards........................................ 107 
4.4 Discussion and Conclusions.

5.1 Introduction

5.2 Data and Methods.

5.2.1 Data.

5.2.2 Methods for identifying extreme wet, dry, neutral and wet-dry events.

5.2.3 Wet-dry metrics.

5.2.4 Correlation tests. 122

5.3 Results.... 122

5.3.1 Land area impacted by extreme wet, dry, neutral and wet-dry events .122

5.3.2 Concurrent global flood and drought events .125

5.3.3 Wet-dry (WD) ratio. 128

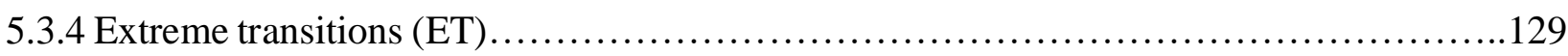

5.3.5 Correlations with Climate Indices.............................................. 132

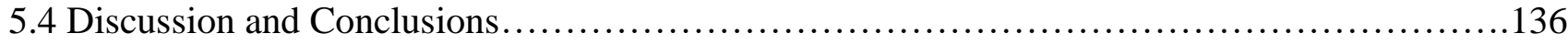

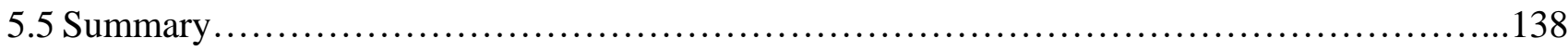

Chapter 6: Discussion..................................................................140

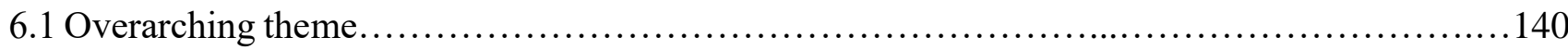

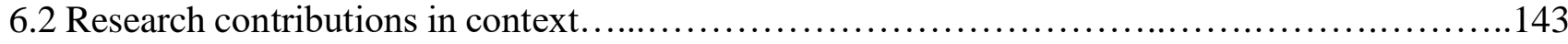

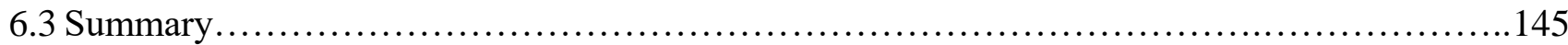

Chapter 7: Conclusions................................................................147

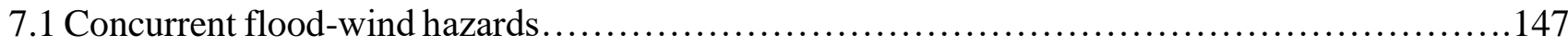

7.2 Weather pattern persistence and multi-hazards........................................ 149

7.3 Concurrent wet and dry hydrological extremes......................................... 151

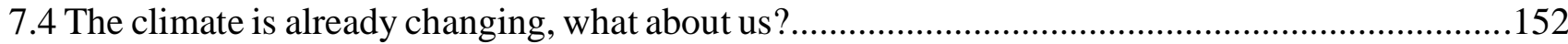

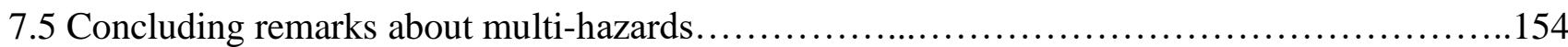

Annex 1..................................................................................156

A.1 Supplementary Information Chapter 3........................................156 


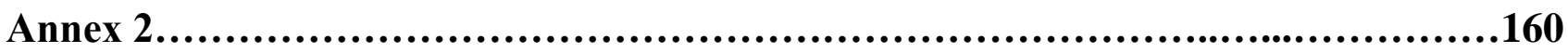

A.2 Supplementary Information Chapter 4..............................................160

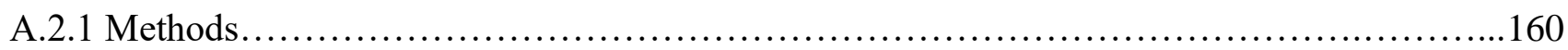

A.2.1.1 CMIP5, reanalyses and Lamb's catalogue...................................160

A.2.1.2 Statistical methods and analyses............................................ 161

A.2.1.2.1 2-day persistence............................................... 161

A.2.1.2.2 Seasonal trends................................................... 162

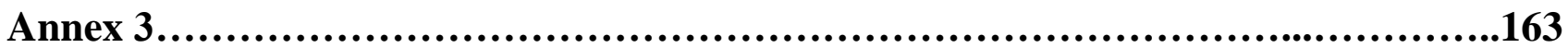

A.3 The published article within the journal Environmental Research Letters - Chapter 3 of this thesis.....................................................................163

References..........................................................................176 


\section{Glossary}

\begin{tabular}{|c|c|}
\hline 20CR & Twentieth Century Reanalysis \\
\hline A & Area \\
\hline AA & Arctic Amplification \\
\hline AMAX & Annual maxima \\
\hline AMIN & Annual minima \\
\hline AMO & American Multidecadal Oscillation \\
\hline AOGCMs & $\begin{array}{c}\text { Atmopshere-Ocean General Circulation } \\
\text { Models }\end{array}$ \\
\hline ARs & Atmospheric Rivers \\
\hline BI & British Isles \\
\hline CCRA & $\begin{array}{c}\text { UK Climate Change Risk Assessment } \\
\text { Evidence Report }\end{array}$ \\
\hline $\mathrm{CDF}$ & Cumulative distribution function \\
\hline CMIP5 & $\begin{array}{c}\text { Coupled Model Intercomparison Project } \\
\text { Phase } 5\end{array}$ \\
\hline Concurrent hydroclimatic hazards & $\begin{array}{l}\text { The co-occurrence in time of two or more } \\
\text { hydroclimatic hazards in a given } \\
\text { geographical region (e.g. river floods and } \\
\text { storms in Great Britain during boreal } \\
\text { winter) or in different geographically- } \\
\text { remote regions (e.g. river floods in Australia } \\
\text { ad drought in the middle-East during } \\
\text { December). }\end{array}$ \\
\hline DJF & December January February \\
\hline DRR & Disaster Risk Reduction \\
\hline EA & Environment Agency \\
\hline EA pattern & East Atlantic pattern \\
\hline ENSO & El Niño Southern Oscillation \\
\hline ET & Extreme transition \\
\hline ETCs & Extra-tropical cyclones \\
\hline
\end{tabular}




\begin{tabular}{|c|c|}
\hline EVT & Extreme value theory \\
\hline F-Index & Flood Index \\
\hline FY & Flood yield \\
\hline GB & Great Britain \\
\hline GCM & Global Climate Model \\
\hline GDP & Gross domestic product \\
\hline HAs & Hydrometric Areas \\
\hline IPCC & Intergovernmental Panel on Climate Change \\
\hline IVT & Integrated vapour transport \\
\hline JJA & June July August \\
\hline $\mathrm{Jt}$ & Joining time \\
\hline $\mathrm{L}$ & Window length \\
\hline LWTs & Lamb Weather Types \\
\hline MAM & March April May \\
\hline MBF & Multi-basin flooding \\
\hline $\mathrm{mFY}$ & Multi-basin Flood Yield \\
\hline MME & Multi-model ensemble \\
\hline MMEM & Multi-model ensemble mean \\
\hline Multi-hazards or Compound hazards/events & $\begin{array}{l}\text { The generic definition of two or more } \\
\text { natural hazards interacting in time and } \\
\text { space. }\end{array}$ \\
\hline NAO & North Atlantic Oscillation \\
\hline NCEP/NCAR & $\begin{array}{c}\text { National Centers for Environmental } \\
\text { Predictions/National Center for } \\
\text { Atmospheric Research }\end{array}$ \\
\hline NOAA & $\begin{array}{c}\text { National Oceanic \& Atmospheric } \\
\text { Administration }\end{array}$ \\
\hline PDO & Pacific Decadal Oscillation \\
\hline PNA & Pacific-North American pattern \\
\hline QBO & Quasi-Biennial Oscillation \\
\hline RCM & Regional Climate Model \\
\hline RCPs & Representative Concentration Pathways \\
\hline
\end{tabular}




\begin{tabular}{|c|c|}
\hline sc_PDSI_pm & $\begin{array}{c}\text { Self-calibrated Palmer Drought Severity } \\
\text { Index (Penman-Monteith model) }\end{array}$ \\
\hline SLP & Sea-level pressure \\
\hline SON & $\begin{array}{c}\text { September October November } \\
\text { Evapotranspiration Index }\end{array}$ \\
\hline SPEI & Standardized Precipitation Index \\
\hline SPI & Sea-surface temperature \\
\hline SST & Total Drained Area \\
\hline TDA & Time to peak \\
\hline Tp & Urban Heat Island \\
\hline UHI & United Kingdom \\
\hline UK & Reduction \\
\hline UNDRR & The United States of America \\
\hline USA & Very Severe Gales \\
\hline VSGs & Wet-Dry ratio \\
\hline WD-ratio & \\
\hline & \\
\hline & \\
\hline & \\
\hline
\end{tabular}




\section{List of Figures}

Figure 1.1 Thesis structure

Figure 2.1 Literature review sections' links with research chapters .29

Figure 3.1 Network of hydrological stations and related basin areas used in the analyses. The 261 nonnested gauges were selected from an initial network of 649 (Annex A.1.1 Figure S3.1) based on record length, and geographic coverage. Some areas are under-represented (e.g. east England) because they are either ungauged or do not have data for the 1975-2014 period.....

Figure 3.2 Distribution of basins contributing to the extreme MBF episodes in GB during 1975-2014 for six time window lengths (L) (event set E). The maps show respectively: (a) $\mathrm{L}=1$-day $\left(\mathrm{d}_{\max }=\right.$ 27/12/1979); (b) L = 2-days (dmax = 30/10/2000); (c) L = 4-days (dmax = 01/01/2003); (d) L = 6-days

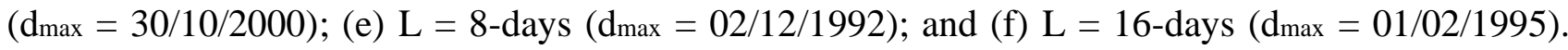
Flood Yield (FY) is a severity metric that represents each basin's peak flow AMAX normalized by the relative basin area. .72

Figure 3.3 Characteristics of the extreme MBF episodes (event set E), compared to event sets A, B, C and D. (a) Maximum number (i.e. $\mathrm{n}_{\mathrm{g}}$ ) of basins with concurrent AMAX versus window length L, defining the most extreme episodes (i.e. event set E); (b) as in (a) but for the total study area affected (i.e. TDA); (c) temporal distribution of peak flow AMAX occurrences for the extreme episodes in event sets A-E; and (d) frequency of LWTs associated with event sets A-E with respect to their expected occurrence, calculated as a Flood Index (Wilby and Quinn, 2013). Significance was determined using the Binomial test. LWTs shown are based on event set E; event sets A-D also contain other LWTs (Annex A.1.1 Figure S3.2).

Figure 3.4 Distribution and relative frequency of occurrence of AMAX within event sets B, C, D and E. (a) Event set B; (b) event set C; (c) event set D; and (d) event set E. The colour scale is a ratio (i.e. from 0 to 1) of AMAX occurrences in a given basin relative to the basin with the largest number in that panel, with dark colours indicating most occurrences 
Figure 3.5 Mean Standardized Precipitation Index (SPI) for episodes within event set B for each window length (L) and SPI time scale (24-1 Months). Green lines are episode SPI averages and the black line represents the overall (40-year, 1975-2014) SPI average i.e. zero by definition. All episodes have SPI that are significantly different from the long-term mean at $99 \%$ level (t-test, not paired)......81

Figure 3.6 Number of Very Severe Gales (VSGs) versus extreme multi-basin Flood Yield (mFY) episodes belonging to event set $\mathrm{C}$ for each water year (1975-2014). Black circles $=12-\mathrm{Month}$ SPI $<-$ 0.3; Grey circles $=-0.3<12$-Month SPI $<0.4$; White circles $=12$-Month SPI $>0.4 . \mathrm{n}=20$ represents the $50 \%$ most extreme VSG and $\mathrm{n}=12$ the $30 \%$ most extreme mFY episodes

Figure 4.1 Grid points used to calculate Jenkinson flow and vorticity terms for the British Isles (BI). Numbers refer to those points used in Equations 4.1 to 4.5

Figure 4.2 Persistence of the seven main LWTs plus unclassified (U) type under RCP8.5. Persistence is calculated for (a) summer, (b) autumn, (c) winter and (d) spring, for the historical 1980s period (1971-2000) and under RCP8.5 by the 2020s (2011-2040), 2050s (2041-2070) and 2080s (2071-2100). Boxplots show distributions of persistence in each LWT, for the 10-member AOGCM ensemble, compared with 20CR, NCEP and the Lamb's catalogue. Segments show the minimum, 1 st quartile, median, 3rd quartile and maximum. Outliers are shown by dots

Figure 4.3 Persistence of the seven main LWTs plus unclassified (U) type under RCP4.5. Persistence is calculated for (a) summer, (b) autumn, (c) winter and (d) spring, for the historical 1980s period (1971-2000) and under RCP4.5 by the 2020s (2011-2040), 2050s (2041-2070) and 2080s (2071-2100). Boxplots show distributions of persistence in each LWT, for the 10-member AOGCM ensemble, compared with 20CR, NCEP and the Lamb's catalogue. Segments show the minimum, 1 st quartile, median, 3rd quartile and maximum. Outliers are shown by dots 101

Figure 4.4 Persistence of selected LWTs and seasons for individual AOGCMs under RCP8.5. (a) Atype (summer), (b) C-type (autumn), (c) W-type (winter) and (d) S-type (spring) in the 1980s compared with the 2020s, 2050s and 2080s under RCP8.5. Persistence is shown for individual AOGCMs alongside the MMEM, 20CR, NCEP and Lamb's catalogue. Asterisks (*) show model runs with persistence outside the $95 \%$ confidence intervals of the boot-strapped $(n=1,000)$ estimates for the 1980s, shown here as black T-bars 103 
Figure 4.5 Persistence of selected LWTs and seasons for individual AOGCMs under RCP4.5. (a) Atype (summer), (b) C-type (autumn), (c) W-type (winter) and (d) S-type (spring) in the 1980s compared with the 2020s, 2050s and 2080s under RCP4.5. Persistence is shown for individual AOGCMs alongside the MMEM, 20CR, NCEP and Lamb's catalogue. Asterisks (*) show model runs with persistence outside the $95 \%$ confidence intervals of the boot-strapped $(n=1,000)$ estimates for the 1980s, shown here as black T-bars .104

Figure 4.6 Projected annual frequencies for selected LWTs and seasons under RCP8.5. Frequencies are shown as MMEM for (a) summer anticyclonic A, (b) autumn cyclonic C, (c) winter westerly W and (d) spring southerly S LWTs under RCP8.5 (2006-2100). MMEM trends are statistically significant at the $1 \%$ level (p-value $<0.01$, modified Mann-Kendall test). Shaded areas represent the 95\% confidence intervals of the MMEM. The trend lines refer to the Sen's slopes calculated with the modified Mann-Kendall test 105

Figure 4.7 Projected annual frequencies for selected LWTs and seasons under RCP4.5. Frequencies are shown as MMEM for (a) summer anticyclonic A, (b) autumn cyclonic C, (c) winter westerly W and (d) spring southerly S LWTs under RCP4.5 (2006-2100). MMEM trends are statistically significant at the $1 \%$ and $5 \%$ levels (p-value $<0.01$ and $<0.05$, modified Mann-Kendall test). Shaded areas represent the $95 \%$ confidence intervals of the MMEM. The trend lines refer to the Sen's slopes calculated with the modified Mann-Kendall test. 106

Figure 4.8 F-Score for LWTs associated with concurrent flood-wind hazards during winter DJF. The F-Score is shown per each CMIP5 AOGCM, MMEM, NCEP, 20CR and Lamb's subjective catalogue for the 1980s, 2020s, 2050s and 2080s periods under RCP8.5. The LWTs used for calculating the FScore are associated with concurrent multi-basin floods and wind hazards within Great Britain (GB) (De Luca et al., 2017). The 1980s MME F-Score were estimated from the mean of $n=1,000$ bootstrapped samples. The AOGCMs 1980s confidence intervals bars are not shown for simplicity because they are vanishingly narrow. 108

Figure 4.9 F-Score for LWTs associated with concurrent flood-wind hazards during winter DJF. The F-Score is shown per each CMIP5 AOGCM, MMEM, NCEP, 20CR and Lamb's subjective catalogue for the 1980s, 2020s, 2050s and 2080s periods under RCP4.5. The LWTs used for calculating the FScore are associated with concurrent multi-basin floods and wind hazards within Great Britain (GB) 
(De Luca et al., 2017). The 1980s MME F-Score were estimated from the mean of $n=1,000$ bootstrapped samples. The AOGCMs 1980s confidence intervals bars are not shown for simplicity because they are vanishingly narrow. 109

Figure 4.10 UHI in tenths of ${ }^{\circ} \mathrm{C}$ for LWTs associated with concurrent heat-air pollution hazards during summer JJA. The UHI is shown per each CMIP5 AOGCM, MMEM, NCEP, 20CR and Lamb's subjective catalogue for the 1980s, 2020s, 2050s and 2080s periods under RCP8.5. The 1980s MME UHI were estimated from the mean of $n=1,000$ boot-strapped samples. The AOGCMs 1980s confidence intervals bars are not shown for simplicity because they are vanishingly narrow..... 110

Figure 4.11 UHI in tenths of ${ }^{\circ} \mathrm{C}$ for LWTs associated with concurrent heat-air pollution hazards during summer JJA. The UHI is shown per each CMIP5 AOGCM, MMEM, NCEP, 20CR and Lamb's subjective catalogue for the 1980s, 2020s, 2050s and 2080s periods under RCP4.5. The 1980s MME UHI were estimated from the mean of $n=1,000$ boot-strapped samples. The AOGCMs 1980s confidence intervals bars are not shown for simplicity because they are vanishingly narrow

Figure 5.1 Percentage (\%) of total land area with (a) wet (blue), (b) dry (red) extremes, (c) neutral (black) and (d) extreme wet + extreme dry (orange) events over the 1950-2014 period. Wet extremes are sc_PDSI_pm $\geq 3$ ) and dry extremes sc_PDSI_pm $\leq-3$ monthly observations. Sen's slopes and the significance of the Mann Kendall test (p-values) are shown in each panel... 124

Figure 5.2 (a) Most widespread extreme global wet hydrological event (blue colour) and coincident extreme dry areas (red colour), December 2010. The event was also the most widespread concurrent wet-dry episode. The percentage $(\%)$ of total land area is shown for both wet and dry extremes, along with the values of the three climate indices (i.e. Niño3.4, PDO and AMO) in December 2010. (b) As (a) but for the most widespread extreme global dry hydrological event, January 2003. (a)-(b) The analysis is based on the self-calibrated monthly mean Palmer Drought Severity Index (sc_PDSI_pm) for the period $1950-2014$

Figure 5.3 Wet-dry (WD) ratio derived for every grid-cell. Blue colours (WD-ratio > 0) mean that the area experienced more wet than dry hydrological extremes. Red colours (WD-ratio < 0 ) indicate the opposite. 
Figure 5.4 Extreme transition (ET) time intervals between extreme wet to dry (blue) and between extreme dry to wet (red). (a) ET as a function of the total percentage (\%) of total land area impacted and (b) cumulative distribution functions (CDFs). The horizontal black line in (b) indicates the 50th quantile (i.e. median) of the distribution and the blue and red lines the respective ET time intervals. The two distributions show a statistically significant difference in their means (p-value $<<0.01$, MannWhitney-Wilcoxon test). 130

Figure 5.5 Extreme transition (ET) time intervals between extreme wet to wet (blue) and between extreme dry to dry (red). (a) ET as a function of the total percentage (\%) of total land area impacted and (b) cumulative distribution functions (CDFs). The horizontal black line in (b) indicates the 50th quantile (i.e. median) of the distribution and the blue and red lines the respective ET time intervals. The two distributions show a statistically significant difference in their means ( $\mathrm{p}$-value $<<0.01$, MannWhitney-Wilcoxon test). To note that for simplicity only ET with a time interval $\leq 200$ months are shown. 131

Figure 5.6 Correlations between extreme wet (sc_PDSI_pm $\geq 3$ ) and dry (sc_PDSI_pm $\leq$-3) hydrological events and (a) Niño3.4, (b) PDO and (c) AMO. For (b) and (c) partial correlations are performed to remove the Niño3.4 signal. Correlations and partial correlations make use of the Spearman's correlation coefficient. Correlations significant at the 5\% level (p-value <0.05) are shown by stippling. The Bonferroni correction was applied to all p-values. In (b) and (c) the ENSO signal has been removed via partial correlations 134

Figure 5.7 Correlations between extreme wet (sc_PDSI_pm $\geq 3$ ) and dry (sc_PDSI_pm $\leq$-3) hydrological events and (a) NAO, (b) PNA and (c) QBO. Correlations and partial correlations make use of the Spearman's correlation coefficient. Correlations significant at the $5 \%$ level (p-value $<0.05$ ) are shown by stippling. The Bonferroni correction was applied to all p-values .135

Figure 6.1 Annual number of Google Scholar outputs based on the keyword 'Multi-Hazard'.... 140

Figure S3.1 Initial hydrological network of 649 gauges. The yellow stations are the 261 non-nested basins used in the analyses, whereas blue stations represent the remaining 388 nested stations excluded from the study because they are located upstream from a non-nested gauge. 156 
Figure S3.2 Lamb Weather Types' (LWTs) observed percentages of occurrence for all event sets (AE). (a) Event set A; (b) event set B; (c) event set C; (d) event set D; and (e) event set E. All with replicated dates excluded.

Figure S3.3 Lamb Weather Types' F-Index (Wilby and Quinn, 2013) calculated for event sets B, C, $\mathrm{D}$ and $\mathrm{E}$ with respect to single-basin occurrences (i.e. event set A). Significance was determined using Binomial test, but with event set A used as expected values. LWTs shown are based on event set E; event sets B-D also contain other LWTs .158

Figure S3.4 Extreme multi-basin flooding episodes' joining times (event set E). (a) L=1-day (dmax $=$ 27/12/1979); (b) L = 2-days (dmax = 30/10/2000); (c) L = 4-days (dmax = 01/01/2003); (d) L = 6-days

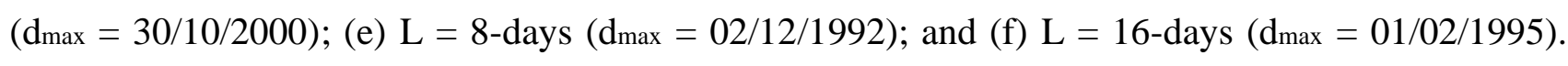
Days are ordered chronologically (e.g. Day $=16$ represents $\mathrm{d} \max$ for $\mathrm{L}=16$-days) 159 


\section{List of Tables}

Table 2.1 Main terminology used in the thesis.

$30-31$

Table 2.2 Classification of natural hazards .33

Table 2.3 Relationships between natural hazards. .34

Table 2.4 Types of interactions and coincidence between natural hazards .35

Table 2.5 Anthropogenic processes affecting the triggering of one or more natural hazards 36

Table 2.6 Interactions between human activities and natural hazards 37

Table 3.1 Extreme MBF episodes in GB during 1975-2014 (event set E). Observations are derived from 19 time windows up to 18 days prior dmax; see main text for details. (a) Window length (L) in days; (b) Total drained area (TDA, $\mathrm{km} 2$ ) involved in each episode (i.e. sum of the area of all involved basins); (c) Percentage of TDA of the 261 basins affected by each episode; (d) Percentage of GB land area affected by each episode; (e) Dates of episodes, where the top row represents $\mathrm{d}_{\max }$; (f) Number of basins with peak flow AMAX registered on the same day; (g) Total number of basins with peak flow AMAX per episode; (h) Percentage of total number of basins (out of 261) with concurrent AMAX per episode; (i) Daily LWT; (j) Average joining time, within an episode, for larger basins (A $\geq 1,000 \mathrm{~km} 2)$; (k) Average joining time for small basins $(A<1,000 \mathrm{~km} 2)$. In $(\mathrm{j})$ and $(\mathrm{k})$ uncertainties are 1 standard error of the mean $79-80$

Table 4.1 CMIP5 multi-model sub-ensemble (MME) used in the analyses. The columns show the: (1) CMIP5 model name; (2) research institute where the model was developed; (3) resolution as latitude by longitude in degrees; and (4) ensemble member analysed. For all models the historical and RCP8.5 (and RCP4.5) sea-level pressure (SLP) outputs are used to calculate daily LWTs for the BI .95

Table 4.2 MME statistical significance of LWTs persistence for RCP8.5. Time periods considered are the 1980s compared to the 2020s, 2050s and 2080s under RCP8.5 during all seasons: summer JJA, autumn SON, winter DJF and spring MAM. Values shown are the W-statistic from the Mann-Whitney- 
Wilcoxon two-tailed test. Statistically significant values (p-value <0.1) are shown in bold.

Table 4.3 MME statistical significance of LWTs persistence for RCP4.5. Time periods considered are the 1980s compared to the 2020s, 2050s and 2080s under RCP4.5 during all seasons: summer JJA, autumn SON, winter DJF and spring MAM. Values shown are the W-statistic from the Mann-WhitneyWilcoxon two-tailed test. Statistically significant values (p-value <0.1) are shown in bold. 102

Table 4.4 Sen's slopes of MMEM seasonal LWTs frequencies for RCP8.5 and RCP4.5. The slopes are calculated using a modified Mann-Kendall trend test over the 2006-2100 period. Four LWTs are shown: anticyclonic (A) for summer JJA; cyclonic (C) autumn SON; westerly (W) winter DJF and southerly (S) spring MAM. MMEM statistical significance is shown as * p-value $<0.05$ and ** p-value $<0.01$ 107

Table 6.1 Suggested open research questions within the field of multi-hazards. .142 


\section{Chapter 1 \\ Introduction}

Weather, climate and hydrological extremes around the world pose significant socio-economic threats and a general consensus is that they will become even more extreme due to anthropogenic climate change (IPCC, 2018).

Within a warmer world, an increase in extreme precipitation events is expected (Chan et al., 2014; Fischer and Knutti, 2016; IPCC, 2018, 2012; Lenderink and Fowler, 2017; Liu and Allan, 2013; Min et al., 2011) because of a larger availability of water vapour that generates from an increased water holding capacity of the atmosphere (Trenberth, 2011). Such increases in precipitation extremes may also eventually lead to more frequent and/or severe flooding events (Arnell and Gosling, 2016; IPCC, 2012), also accompanied by a shift in the timing of floods (Blöschl et al., 2017) and projected rising global flood risk in the future (Winsemius et al., 2016). Moreover, a shift in the global mean temperature, is expected to translate into more extreme heatwaves with related human heat-stress projected to impact our everyday lives and businesses (IPCC, 2018; Matthews et al., 2017; Rahmstorf and Coumou, 2011). There is also medium confidence that some regions in the world are expected to experience more severe and longer droughts (Dai, 2012; IPCC, 2018; Liu and Allan, 2013; Prudhomme et al., 2014; Trenberth et al., 2013) and even tropical cyclones may become more intense, with their frequency unchanged or even decreased (Emanuel, 2005, 2013; IPCC, 2012; Knutson et al., 2010; Oouchi et al., 2006; Sobel et al., 2016; Webster et al., 2005).

Changes in extreme events also increase their associated economic damages, with an average annual losses from 1980 ranging from a few US\$ billion to about 354 US\$ billion, the latter reached in 2011, the costliest year ever recorded (IPCC, 2012; Kates et al., 2006; Munich Re, 2017a). Studies also show that most of the increase in damages were due to societal changes and not to changes in extreme events, (e.g. Changnon et al., 2000; Pielke et al., 2008; Weinkle et al., 2012). Flooding events around the world had significant impacts, with 5,725 events causing 220,477 fatalities and economic losses of 1,007 US\$ billion over the period 1980-2017 and with the vast majority of these occurring in Asia (Munich Re, 2017a). On the other hand, heatwaves and wildfires, within the same time-period, caused less economic damages (129 US\$ billion) and were also fewer in number with 992 events recorded by Munich Re. However, the number of heat-related fatalities $(\sim 165,000)$ were almost as high as those for flooding (Munich Re, 2017a), although these numbers may slightly change depending on the 
database selected. The number of winter storms, for example extra-tropical cyclone (ETC), events across the globe amounts to 1,232 with impacts mainly affecting western and central Europe, eastern, central and western United States (USA) and south-east Asia, for a total of 332 US\$ billion losses and 28,162 fatalities over the 1980-2017 period (Munich Re, 2017a).

On the other hand, other studies argue that no trends in losses are found when data are normalised by societal changes (Changnon et al., 2000; Crompton et al., 2011; Crompton and McAneney, 2008; Pielke et al., 2008; Weinkle et al., 2012). For instance, Crompton et al. (2011) investigated how much time is needed for US tropical cyclone losses to be attributed to anthropogenic climate change and found that depending on the Global Climate Model (GCM) used the emergence of such a signal spans between 120 to 550 years. In a second study, Crompton and McAneney (2008) normalised Australian insured losses from meteorological hazards and found no trends that could be attributed to anthropogenic climate change. Weinkle et al. (2012) constructed a global database of tropical cyclone landfalls and found no increasing trends in the frequency and intensity of tropical cyclones. They concluded that the observed increasing losses associated with tropical cyclones are to be attributed by increasing wealth in areas affected by cyclones' landfall. Hence, investigating such hazards and their associated socio-economic impacts, and possible links to anthropogenic climate change, is a significant topic for enquiry.

A significant body of research is being devoted to weather, climate and hydrological extremes and risks. This literature spans physical processes, from possible dynamical mechanisms linked to Arctic Amplification (Screen and Simmonds, 2010) that can exacerbate mid-latitude weather and climate extremes (e.g. Coumou et al., 2018) to disentangling the contribution of thermodynamics and dynamics to precipitation extremes (Pfahl et al., 2017). Then there is work on the socio-economic dimensions, for example, how El Niño influences global flood risk (Ward et al., 2014b) and observed trends in regional flood risk (Slater and Villarini, 2016). Adaptation measures to extreme events are widely considered too, from strategies to better manage flood risk under climate change (Wilby and Keenan, 2012) to a newly proposed research framework for natural hazards and associated vulnerabilities (Di Baldassarre et al., 2018). Last but not least, possible future changes of weather and climate extremes currently play a major role in advising decision makers and stakeholders, with global climate projections of temperature and precipitation extremes (Fischer et al., 2013; Fischer and Knutti, 2015; Fischer and Schär, 2010). All these studies once again confirm the urgency to address and solve climate-related issues, for the benefit of societies and economies around the world. 
Hydroclimatology is the study of how the climate system is having an influence on the hydrological cycle as well as how weather, climate and hydrological extremes (such as floods, storms, droughts and heatwaves) are impacting or might impact society. Moreover, since weather, climate and hydrological extremes can be considered a significant part of hydroclimatology (and natural hazards), it is also possible to investigate how these phenomena interact with each other and of course, how they interact with the climate system itself. Broadly speaking, in the past two decades or so research looking at interacting natural hazards has grown considerably, such that the new sub-field of multi-hazards (or compound hazards) has emerged (Asprone et al., 2010; Bovolo et al., 2009; Gill and Malamud, 2014; Grünthal et al., 2006; Hillier et al., 2015; Kappes et al., 2012a; Perry and Lindell, 2008; Terzi et al., 2019; Zscheischler et al., 2018). An example of a multi-hazard event could be for instance the generation of lahars (the mobilisation of ash and tephra deposits due to rainfall) on an active volcano flanks in Guatemala, that eventually trigger flooding as these deposits add sediments into the hydrological system (Harris et al., 2006).

The United Nations (UN) Sendai Framework for Disaster Risk Reduction (UNDRR, 2015) highlights the importance of multi-hazard approaches to disaster risk reduction (DRR) (e.g. early warning systems) at global, regional, national and local levels. Multi-hazard is defined by UNDRR as $i$ ) the variety of multiple major hazards that a country faces and ii) the context by which these perils may occur simultaneously, one after the other (i.e. sequentially), or cumulatively over time, by considering also their potential interrelated effects (UNDRR, 2016). Thus, the investigation of concurrent hydroclimatic hazards could bring significant benefits to societies and economies, including improved adaptation strategies for vulnerable societies and increased economic resilience to disasters. For instance, national risk assessments could be extended to multi-risk assessments, considering multiple natural hazards and their associated vulnerability and exposure components not as independent features but as processes that can interact over time, such as interacting fluvial floods and cyclone storm surges in mega-delta regions (Ikeuchi et al., 2017; Ward et al., 2018), ETCs bringing combined severe winds and multi-basin flooding episodes (De Luca et al., 2017) and earthquakes eventually triggering landslides, tsunamis and floods (Kargel et al., 2016; Suleimani et al., 2009).

Multi-hazards research can also bring benefit to global insurance and re-insurance industries, as the premium paid by households and businesses may only cover single-hazard events, without offering the possibility to be insured for two or more hazards concurrently impacting an area in a given short time-window (e.g. flooding with severe winds, De Luca et al., 2017), or longer periods (e.g. wet-dry fluctuations leading to shrink-swell subsidence events, Collet et al., 2018; Harrison et al., 2012; 
Pritchard et al., 2015). This is significant because the insurance provider may not have set aside sufficient funds to cover for losses generated by interacting hazards as, for example, flood and wind damages may fall under the same insurance claim (Hillier et al., 2015).

The over-arching question of this thesis is: How one can measure concurrent hydroclimatic hazards at different time and spatial scales? The answer is given through three studies that investigate weather, climate and hydrological extremes using a diverse set of methodologies and data. The time scales used in the studies belong to both past and future. For the former, observational data, from the 1950s to 2014 are used, whereas for the latter future climate projections up to 2100 are gathered and analysed. The spatial scales, on the other hand, are nested and span from the river catchment unit, to the British Isles (BI) and then eventually to the global scale such that a local, national and global perspective is provided.

The research questions of the study can be summarised as follows:

For concurrent flood and wind hazards between river basins in Great Britain.

$\mathrm{R} 1$ : What is the spatio-temporal distribution of multi-basin flooding episodes?

R2: What are the most frequent weather patterns observed during these widespread floods?

R3: How are multi-basin floods, atmospheric rivers (ARs) and very severe gales (VSGs) linked?

For concurrent hazards linked to persistent weather patterns over the British Isles.

R4: How has persistence in weather pattens changed historically?

R5: To what extent can Atmosphere-Ocean General Circulation Models (AOGCMs) reproduce observed weather pattern persistence over the BI?

R6: How are weather pattern persistence and frequency expected to change in the future under different Representative Concentration Pathways (RCPs)?

R7: How changes in future weather type persistence might translate into changed risk of winter floodwind and summer heatwave-air pollution concurrent hazards?

For concurrent extreme wet and dry hydrological extremes globally.

R8: How observed globally independent and concurrent wet-dry hydrological extreme events changed in the past?

R9: What were the most spatially extensive independent and concurrent wet-dry hydrological extreme events? 
R10: How new metrics can help in better investigate concurrent wet-dry extremes?

R11: How are these extremes related to different modes of climate variability?

Chapter 2 provides a literature review of the three main streams of research to provide the context for later chapters. The first topic addressed is multi-hazards, with an introduction to the subject along with material focussing on floods driven by storms. The multi-hazards literature review is strictly connected to Chapters 3-5, which are introduced below. Then the second topic refers to weather patterns, specifically the Lamb Weather Types (LWTs) (Jones et al., 1993; Lamb, 1972). This links with the previous chapters through a discussion on how possible future changes in LWTs may translate into independent and compound weather and climate extremes. Here the LWTs classification scheme is broadly described with particular focus on the BI, and their links to atmospheric variables (e.g. precipitation, temperature and pollutants). The literature review on LWTs therefore introduces Chapter 4 through a generic overview on the use and impacts of LWTs research. Lastly, the third research stream provides the basis for Chapter 5 which discusses wet-dry hydrological extremes and modes of climate variability. Here, studies investigating wet and dry hydrological extremes and the links between three climate indices and extreme river flows at regional and global scales are reviewed.

The first research area (Chapter 3) addresses the over-arching question of concurrent hydroclimatic hazards by examining multi-hazard (or compound) events (Zscheischler et al., 2018) over GB. Here the investigation examines extreme multi-basin flooding driven by ETCs (De Luca et al., 2017). Chapter 3 offers potential insights for stakeholders, emergency planners and policy makers, with also methods and metrics easily applicable elsewhere in the world. The aim in Chapter 3 is to extend the typical view of fluvial flooding confined to a single river basin, to coherent flooding across multiple river basins within a time-frame of up to two weeks (De Luca et al., 2017; Uhlemann et al., 2010). The chapter then investigates whether such multi-basin flooding events are driven by ETCs impacting the BI. Evidence that extreme multi-basin flooding is linked to ETCs is relevant to stakeholders, insurance industry and emergency managers, as during such events combined flood-wind impacts on large scales may be expected to cause significant socio-economic damages in the absence of adaptation measures.

Chapter 4 addresses the topic of concurrent hydroclimatic hazards by examining future climate projections of weather patterns (LWTs or atmospheric circulation) (Jenkinson and Collison, 1977; Jones et al., 1993; Lamb, 1972) and associated metrics that quantify both independent and multihazards. Here, the connection with the main over-arching research question is addressed from both a qualitative and quantitative perspective by considering how specific synoptic weather patterns can 
translate into local weather, climate and hydrological extremes (e.g. Burt and Howden, 2013; De Luca et al., 2017; Pattison and Lane, 2012). The chapter also investigates how specific LWTs can contribute to concurrent flood-wind hazards and how changes in LWT persistence could affect the nocturnal Urban Heat Island (UHI) of London and hence combined heatwave-poor air quality events. The results of the study provide a methodology based on weather pattern persistence, frequency and multi-hazard metrics that can help improve the understanding of weather and climate risks to a range of vulnerable communities.

Finally, Chapter 5 investigates concurrent hydroclimatic hazards in terms of interacting wet and dry hydrological extremes at the global scale, driven by dominant modes of climate variability. The dataset used to investigate such events is the Palmer Drought Severity Index (PDSI) (Dai et al., 2004) and the climate indices deployed are the Niño3.4 (Rayner et al., 2003; Trenberth, 1997), Pacific Decadal Oscillation (PDO) (Mantua and Hare, 2002) and Atlantic Multidecadal Oscillation (AMO) (Schlesinger and Ramankutty, 1994). Within the study, new metrics for quantifying concurrent wetdry hydrological extremes are also introduced. The results obtained bring new insights about multihazards at the global scale, with also scope for incorporating modes of climate variability into hydrological forecast models. Such findings could benefit stakeholders and companies that rely on global diversified portfolios and provide information for emergency managers about the timing and associated spatial distribution of both independent and concurrent wet and dry extreme events.

These three pillars of the research, although different in nature and methodology, share a common feature which is the quantification of concurrent hydroclimatic hazards at different time and spatial scales. All the three studies investigate multi-hazards, however the second study addresses the main topic from a both a qualitative and quantitative point of view. The commonalities running through the studies are the investigations of natural hazards, that can affect negatively societies and economies independently of the spatial scales considered and the quantification of their interactions through various metrics. Moreover, there is hope that the three studies provide useful and new metrics, information and insights that are valuable for stakeholders, policy makers and insurance companies. The purpose of the differences between the studies is to show that the over-arching topic of concurrent hydroclimatic hazards needs to be addressed from a range of perspectives that draws on a multidisciplinary pool of research techniques and information sources.

Figure 1.1 provides an overview of the thesis structure and links between the research elements which variously address concurrent hydroclimatic hazards. The work here presented is organised as follows: 
a literature review on multi-hazards, weather patterns, wet and dry hydrological extremes and modes of climate variability is presented in Chapter 2; the extreme multi-basin flooding linked to ETCs research in GB follows in Chapter 3; future projections and analysis of persistent weather patterns over the BI as a means of examining future multi-hazards in Chapter 4; globally independent and concurrent wet and dry hydrological extremes driven by modes of climate variability in Chapter 5; then a Discussion of the unifying themes running through the thesis in Chapter 6 along with an assessment of the wider implications of the research; and lastly Conclusions and opportunities for further research are presented in Chapter 7. 


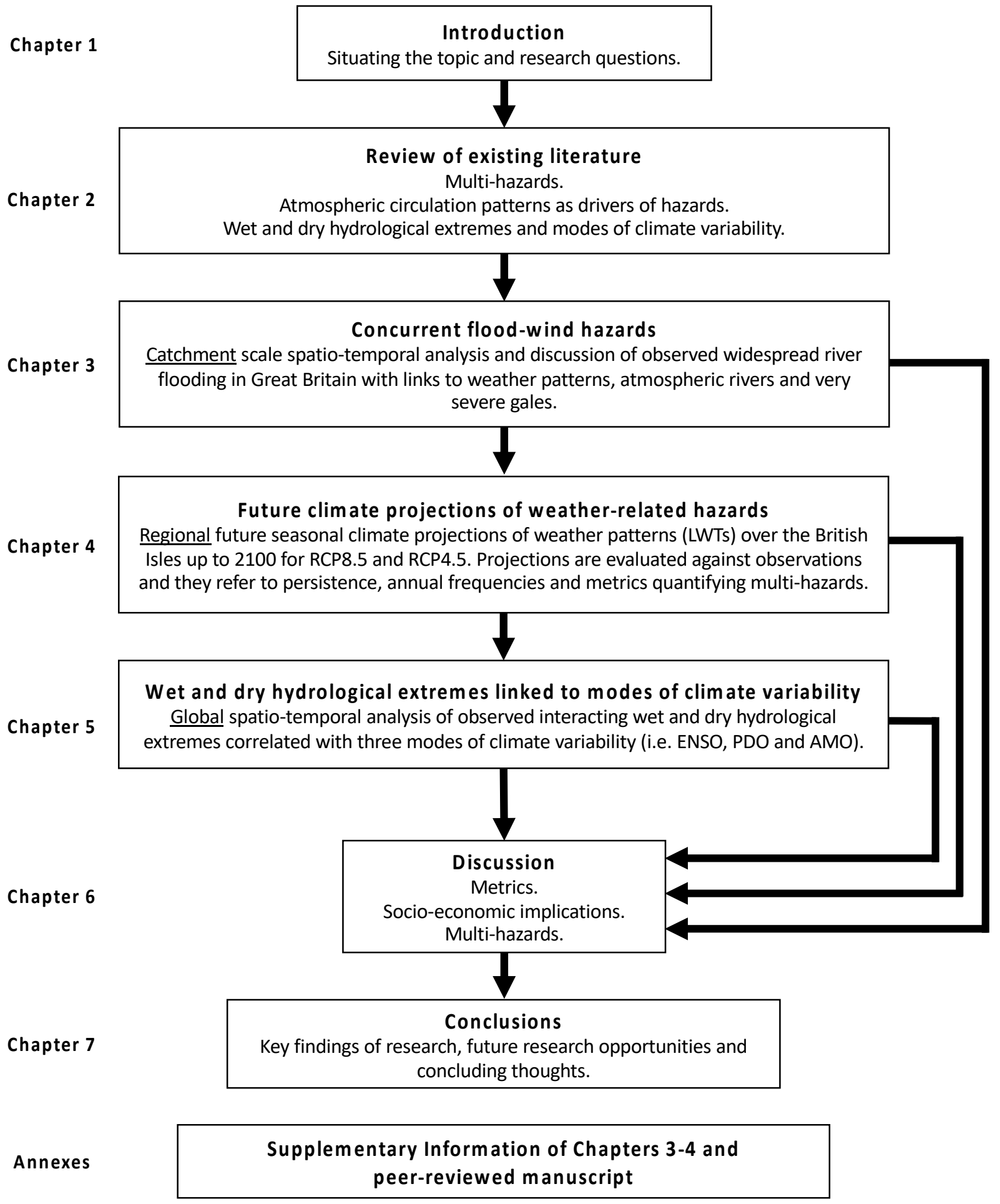

Figure 1.1 Thesis structure. 


\section{Chapter 2}

\section{Literature review}

\subsection{Introduction}

In this Chapter, a literature review on the three main topics covered by this work is presented. Section 2.2 frames to multi-hazards concepts, Section 2.2.1 on multi-hazards and risk assessments and Section 2.2.2 specifically focusses on concurrent floods and wind-storm events. Section 2.3, on the other hand, provides a review of the application of weather pattern (i.e. Lamb Weather Types, LWTs) analysis to hydroclimatic variables and associated natural hazards, across different geographical regions. Lastly, Section 2.4 outlines studies on hydrological extremes (Section 2.4.1) and river flooding linked with modes of climate variability (Section 2.4.2).

The literature review sections refer to research Chapters 3-5 as follows:

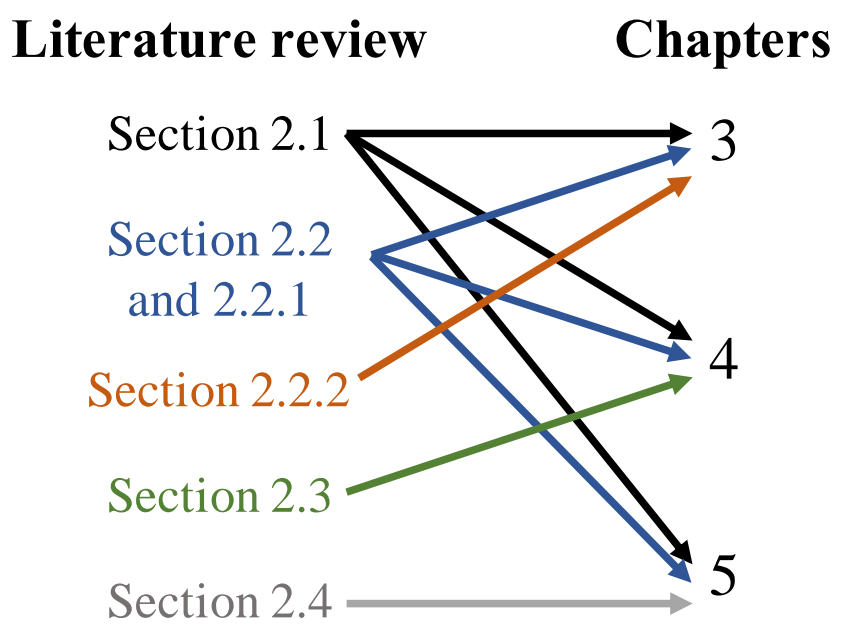

Figure 2.1 Literature review sections' links with research chapters.

To begin with, a few definitions of working terms, taken from UNDRR (2017a, pp. 11-24), are made. The UNDRR is the United Nations Office for Disaster Risk Reduction and therefore it can be considered as the world-leading international organisation that provides policies with respect to disaster risk reduction activities, by implementing the Sendai Framework for Disaster Risk Reduction (UNDRR, 2015). Thus, the following terminology is possibly the most general, correct and exhaustive available at the international and policy level: 


\begin{tabular}{|c|c|}
\hline Disaster & $\begin{array}{l}\text { A serious disruption of the functioning of a community or a society at any scale } \\
\text { due to hazardous events interacting with conditions of exposure, vulnerability } \\
\text { and capacity, leading to one or more of the following: human, material, } \\
\text { economic and environmental losses and impacts. }\end{array}$ \\
\hline Disaster Risk & $\begin{array}{l}\text { The potential loss of life, injury, or destroyed or damaged assets which could } \\
\text { occur to a system, society or a community in a specific period of time, determined } \\
\text { probabilistically as a function of hazard, exposure, vulnerability and capacity. }\end{array}$ \\
\hline $\begin{array}{l}\text { Disaster Risk } \\
\text { Reduction }\end{array}$ & $\begin{array}{l}\text { Disaster risk reduction is aimed at preventing new and reducing existing disaster } \\
\text { risk and managing residual risk, all of which contribute to strengthening } \\
\text { resilience and therefore to the achievement of sustainable development. }\end{array}$ \\
\hline Economic Loss & $\begin{array}{l}\text { Total economic impact that consists of direct economic loss and indirect } \\
\text { economic loss. } \\
\text { Direct economic loss: the monetary value of total or partial destruction of } \\
\text { physical assets existing in the affected area. Direct economic loss is nearly } \\
\text { equivalent to physical damage. } \\
\text { Indirect economic loss: a decline in economic value added as a consequence of } \\
\text { direct economic loss and/or human and environmental impacts. }\end{array}$ \\
\hline Exposure & $\begin{array}{l}\text { The situation of people, infrastructure, housing, production capacities and other } \\
\text { tangible human assets located in hazard-prone areas. }\end{array}$ \\
\hline Hazard & $\begin{array}{l}\text { A process, phenomenon or human activity that may cause loss of life, injury or } \\
\text { other health impacts, property damage, social and economic disruption or } \\
\text { environmental degradation }\end{array}$ \\
\hline Multi-Hazards & $\begin{array}{l}\text { (1) The selection of multiple major hazards that the country faces, and (2) the } \\
\text { specific contexts where hazardous events may occur simultaneously, cascadingly } \\
\text { or cumulatively over time, and taking into account the potential interrelated } \\
\text { effects. }\end{array}$ \\
\hline Preparedness & $\begin{array}{l}\text { The knowledge and capacities developed by governments, response and recovery } \\
\text { organizations, communities and individuals to effectively anticipate, respond to } \\
\text { and recover from the impacts of likely, imminent or current disasters. }\end{array}$ \\
\hline Prevention & Activities and measures to avoid existing and new disaster risks. \\
\hline
\end{tabular}




\begin{tabular}{|l|l|}
\hline Resilience & $\begin{array}{l}\text { The ability of a system, community or society exposed to hazards to resist, } \\
\text { absorb, accommodate, adapt to, transform and recover from the effects of a } \\
\text { hazard in a timely and efficient manner, including through the preservation and } \\
\text { restoration of its essential basic structures and functions through risk } \\
\text { management. }\end{array}$ \\
\hline Vulnerability & $\begin{array}{l}\text { The conditions determined by physical, social, economic and environmental } \\
\text { factors or processes which increase the susceptibility of an individual, a } \\
\text { community, assets or systems to the impacts of hazards. }\end{array}$ \\
\hline
\end{tabular}

Table 2.1 Main terminology used in the thesis.

In this thesis the research focus is on multi-hazards and the other working terms were used mainly in the discussion of the findings, as they are strictly connected to the multi-hazard components. If there were no multi-hazard events, there were no multi-risks and possible disasters with associated economic losses. Here, the term multi-hazards generally refers to: i) floods and storms; ii) drought, heatwaves and air pollution; and iii) wet and dry hydrological extremes. The discussion of these combined hazards takes into account the exposure, preparedness, prevention, vulnerability and resilience of communities living in different geographical areas, from local to global scale. For example, communities and businesses settled in Great Britain (GB) and more generally in the British Isles (BI) are likely exposed to concurrent flood and storm events. On the other hand, people living in the Greater London area have enhanced chances to experience heatwaves and severe air pollution events due to the Urban Heat Island (UHI) effect. Lastly, stakeholders with significant assets invested in global crop production and/or hydropower generation may be affected negatively by the temporal coincidence of widespread flood and drought events in diverse and remote parts of the globe. Therefore, each of these cases requires targeted disaster risk reduction and prevention measures to better increase and reduce resilience and vulnerability with respect to multi-hazard events (UNDRR, 2015).

In this work, different empirical metrics have been introduced with the aim to quantify single and multiple hazards. The use of metrics, for both (multi)hazard/risk quantification, is nowadays common practice (e.g. Cutter et al., 2008; De Luca et al., 2019b; Ekström et al., 2018; Forzieri et al., 2016; Hao et al., 2018; Russo et al., 2015). One of the main advantages of metrics is that they can be useful for translating observed or projected impacts of one or more natural hazards to the wider community, nonexperts included. Therefore, their formulation and description need to be simple, pragmatic and directly connected to the main physical process under investigation. Metrics can also summarise 
complex processes purely defined on a mathematical level, for example in the phase-space, and at the same time provide information about the dynamics of compound hazards (De Luca et al., 2019b; Faranda et al., 2017a; Messori et al., 2017). There is therefore hope that metrics will be eventually used by stakeholders and public agencies to better prepare, communicate and adapt to (multi)hazards/risks. Possible disadvantages of metrics could be their simplicity, i.e. the fact that within their formulation there could be processes and mechanisms not quantified or neglected, and also the possibility that there could be many used to describe the same process. When designing a metric it is therefore important to consider: i) who may be interested in using the metric; ii) if there are already other metrics available in the literature that quantify the physical process under investigation; iii) that the metric is not difficult to interpret; iv) and that directly quantifies the (multi)hazards. In conclusion, the design of a metric is a trade-off between simplicity and correct representation of the (multi)hazards. If it is too simple it may be very easy to be understood by end-users, but it may not be rigorous enough to present the physical process and vice-versa. A similar trade-off is relevant when considering data belonging to different spatial and time scales.

Indeed, this thesis addresses the topic of multi-hazards with a set of investigations (Chapters 3-5) spanning different spatial and time scales. Therefore, multi-hazards occurring at catchment, regional and global geographical scales were investigated by making use of both observations and climate model projections up to 2100. A clear benefit when looking at small-scale geographical areas is that the level of detail one can obtain is much higher compared to regional or global analyses. Thus, the information gained can inform local communities and stakeholders with a smaller level of uncertainty compared to larger-scale analysis. For example, in Chapter 3 the river basins (even the very small ones) involved in widespread flooding linked with extra-tropical cyclones (ETCs) in GB are clearly identified. This could have been much more difficult to detect if, for example, the analysis was conducted by making use of a global hydrological model with a spatial horizontal resolution of $2.5 \mathrm{deg}$ x $2.5 \mathrm{deg}$. On the other hand, a coarser spatial resolution has the benefit to provide a global picture of a given multi-hazards process, with a manageable computational cost. For example, in Chapter 5 concurrent wet and dry hydrological extremes have been explored at the global scale, and although localised details of these concurrent extremes cannot be obtained, one has a global picture of where and when they co-occurred. Thus, such information may not be highly useful for a local community (e.g. village, business or farm) but it can be appreciated by international organizations and global stakeholders. A similar concept applies also to time-scales. Here, a finer temporal resolution of, for example, hourly instead of daily observations can be necessary for detecting a specific physical process (e.g. storm surges or wind gusts). Whereas the output of a climate model, while not providing the exact 
information for a given day in the future, informs us about the possible general trends of the chosen variable at seasonal, annual or decadal scales. In conclusion, both small and large-scale geographical analyses and finer and coarser temporal resolutions have pros and cons, and the choice of one instead of the other depends respectively on the targeted end-user and physical process under investigation. In this thesis it is shown that multi-hazards research can, and needs to, be tackled at both small and large geographical scales, by looking at both observations and future climate projections.

\subsection{Multi-hazards}

Within the academic community, the concept of natural hazards acting independently has now changed to a multi-hazard or compound events approach (UNDRR, 2015; Zscheischler et al., 2018), and although with slower timing this is occurring in the governance sector as well. Thus, a more holistic, multi-hazards perspective is emerging with importance especially for future projections of potential high-impact events and for bridging the gap between physical/social scientists, engineers, climate impact modellers and stakeholders (AghaKouchak et al., 2018; Zscheischler et al., 2018).

One of the most exhaustive classification of natural hazards can be found in the works of Gill and Malamud (2017, 2014), where they divide hazards into six groups:

\begin{tabular}{|c|l|}
\hline Geophysical & $\begin{array}{l}\text { Earthquake, tsunami, volcanic eruption, landslide and snow } \\
\text { avalanche. }\end{array}$ \\
\hline Hydrological & Flood and drought. \\
\hline Shallow Earth Processes & Subsidence and ground collapse. \\
\hline Atmospheric & $\begin{array}{l}\text { Tropical cyclones, tornado, hail, snow, lightning, thunderstorm and } \\
\text { climatic change. }\end{array}$ \\
\hline Biophysical & Wildfire. \\
\hline Space hazards & Geomagnetic storm. \\
\hline
\end{tabular}

Table 2.2 Classification of natural hazards.

In this work the natural hazards investigated belong to the Hydrological and Atmospheric groups. Indeed, in Chapter 3 the interactions between widespread flooding and ETCs are investigated over GB 
(De Luca et al., 2017), whereas in Chapter 4 past and future weather pattern persistence in the BI is linked with flood-wind and heatwave-air pollution hazards (De Luca et al., 2019a). Lastly, in Chapter 5 a global analysis of concurrent wet and dry hydrological extremes with also links to modes of climate variability is presented (De Luca et al., 2019c).

The relationship between hazards types are diverse and these can be, for example, summarised as follows (Kappes et al., 2012a):

\begin{tabular}{|c|l|}
\hline Cascade (or domino effect) & $\begin{array}{l}\text { When the occurrence of a hazard eventually leads to subsequent } \\
\text { hazards events. }\end{array}$ \\
\hline Interactions & A mutual influence between two physical processes. \\
\hline Compound hazards & $\begin{array}{l}\text { When similar hazards act together while exceeding their damage } \\
\text { thresholds (e.g. hail, lightning and wind in a severe storm, Hewitt } \\
\text { and Burton, 1971). }\end{array}$ \\
\hline Multiple hazards & $\begin{array}{l}\text { When different hazards coincide accidentally or following one } \\
\text { another (e.g. floods produced by a hurricane, Hewitt and Burton, } \\
1971) .\end{array}$ \\
\hline
\end{tabular}

Table 2.3 Relationships between natural hazards.

In Chapters 3-5 the research focusses on both compound and multiple hazards, however the thesis generally refers to them as concurrent hazards. Hence, concurrent hazards, i.e. different hazard events that happen within a relative short time-period (e.g. days/weeks) and within a given geographical area, such as widespread flooding and severe wind events happening during an ETC can be interpreted as compound hazards. The same definition applies to concurrent heatwaves, drought and air pollution hazards driven for example by persistent anticyclonic weather during summer. On the other hand, in the case of concurrent wet and dry hydrological extreme events, observed in spatially-remote regions across the globe, the most appropriate definition (as per above) is multiple hazards, as these events may not necessarily have meaningful physical connections.

Gill and Malamud (2014) suggested also that there exist different typologies of interactions and coincidence between hazards: 


\begin{tabular}{|c|l|}
\hline $\begin{array}{c}\text { Interactions where a hazard is } \\
\text { triggered }\end{array}$ & $\begin{array}{l}\text { When a hazard triggers a second similar or different } \\
\text { natural hazard, which it can trigger a third one or more, } \\
\text { escalating the accumulated hazard potential in a region } \\
\text { (e.g. multiple landslides generated by an earthquake, } \\
\text { extreme rainfall or snowmelt). }\end{array}$ \\
\hline \multirow{2}{*}{$\begin{array}{c}\text { Interactions where the probability of } \\
\text { a hazard is increased }\end{array}$} & $\begin{array}{l}\text { When a primary hazard does not trigger a secondary but } \\
\text { it changes some aspect of the environment, increasing its } \\
\text { vulnerability, which will eventually facilitate the } \\
\text { secondary hazard to occur (e.g. in case of a wildfire the } \\
\text { vegetation populating a slope will be lost and as } \\
\text { vegetation improves slopes' stability, a heavy rainfall or } \\
\text { earthquake will be easily able to trigger landslides). }\end{array}$ \\
\hline \multirow{2}{*}{$\begin{array}{c}\text { Interactions where the probability of } \\
\text { a hazard is decreased }\end{array}$} & $\begin{array}{l}\text { This is the opposite situation as the case before (e.g. a } \\
\text { heavy rainfall which increases the surface soil moisture } \\
\text { content and reduces the chances of a wildfire in the } \\
\text { immediate aftermath). }\end{array}$ \\
\hline $\begin{array}{l}\text { When one or more hazards happen in spatial and } \\
\text { temporal proximity to each other. This spatiotemporal } \\
\text { coincidence can be applied to both triggered hazards (i.e. } \\
\text { primary and secondary) and independent hazards. }\end{array}$ \\
\hline
\end{tabular}

Table 2.4 Types of interactions and coincidence between natural hazards.

With respect to this further definition, Chapters 3-5 consider i) Interactions where a hazard is triggered and ii) Events involving the spatial and temporal coincidence of natural hazards. For example, i)-ii) relate to concurrent widespread flooding and severe wind events (Chapters 3-4), as the former hazard is triggered by the latter and they also occur within a close spatio-temporal frame. On the other hand, ii) is connected to spatially-remote but temporally-concurrent global wet and dry hydrological extremes (Chapter 5).

However, one or more natural hazard events are not always triggered by natural/physical forcing. Thus, it is also important to consider how anthropogenic processes are influencing the occurrence of natural hazards and their interactions. Again, Gill and Malamud (2017) presented a broad overview of this 
subject, as they investigated 18 (non-malicious) human process types influencing 21 natural hazards and their interactions. In this thesis the direct human influence on natural hazards is not quantified, therefore the following description is intended to only provide a general overview of the human processes involved.

The 18 anthropogenic processes, which can affect the likelihood of one or more natural hazard to occur, are the following (Gill and Malamud, 2017):

\begin{tabular}{|c|c|}
\hline \multirow[t]{2}{*}{ Subsurface processes } & $\begin{array}{l}\text { Material extraction (groundwater abstraction, oil/gas extraction, } \\
\text { infrastructure construction and mining); }\end{array}$ \\
\hline & Material addition (fluid injection). \\
\hline \multirow{3}{*}{ Surface processes } & $\begin{array}{l}\text { Land use change (vegetation removal, agricultural practice change and } \\
\text { urbanisation); }\end{array}$ \\
\hline & $\begin{array}{l}\text { Material extraction (infrastructure construction and quarrying/surface } \\
\text { mining); }\end{array}$ \\
\hline & $\begin{array}{l}\text { Material addition (infrastructure, infilled ground, reservoir and dam } \\
\text { construction). }\end{array}$ \\
\hline \multirow{3}{*}{$\begin{array}{l}\text { Subsurface and } \\
\text { surface processes }\end{array}$} & Hydrological change (drainage/dewatering and water addition); \\
\hline & Explosion (chemical and nuclear); \\
\hline & Combustion (fire). \\
\hline
\end{tabular}

Table 2.5 Anthropogenic processes affecting the triggering of one or more natural hazards.

The natural hazards considered in this work can be affected by both subsurface and surface anthropogenic processes. This applies particularly to hydrological hazards, such as floods and droughts, where for example groundwater abstraction, land use change and hydrological change can significantly affect the frequency, magnitude and timing of those perils.

The types of interactions, between human activities and natural hazards, which were formulated by Gill and Malamud (2017) are two: 


\begin{tabular}{|c|l|}
\hline \multirow{3}{*}{$\begin{array}{c}\text { Anthropogenic triggering } \\
\text { which can eventually trigger a second one in a cascading effect (e.g. } \\
\text { unloading of slopes which can trigger landslides, eventually leading } \\
\text { to river flooding). }\end{array}$} & $\begin{array}{l}\text { When an anthropogenic process triggers one primary natural hazard, } \\
\text { Anthropogenic } \\
\text { catalysis/impedance }\end{array}$ \\
\hline $\begin{array}{l}\text { When human activities catalyse natural hazard interactions (e.g. } \\
\text { removal of vegetation on a slope likely enhance the chances of rain- } \\
\text { triggered landslides). On the other hand, anthropogenic activities } \\
\text { may also decrease the chances or impede the triggering of a natural } \\
\text { hazard (e.g. vegetation removal may avoid the occurrence of wildfire } \\
\text { triggered by lightning). The catalysis and impedance effects may } \\
\text { both occur before, simultaneously and/or after a primary natural } \\
\text { hazard. }\end{array}$ \\
\hline
\end{tabular}

Table 2.6 Interactions between human activities and natural hazards.

As mentioned before, in Chapters 3-5 the addressed natural hazards are not directly triggered by human activities, however these could both enhance and reduce the chances of their occurrences. For example, engineering structures such as levees are intended to reduce the risk of flooding and water reservoirs should help with respect to agricultural drought events. On the other hand, increased urbanisation may enhance the chances of heatwaves and air pollution events during extreme heat periods.

Other reviews focussing on multi-hazards are provided by Tilloy et al. (2019) and Leonard et al. (2014). Whereas reviews on multi-risk assessments can be found in the works of Gallina et al. (2016) and Terzi et al. (2019). In the former, possible effects of anthropogenic climate change on multi-risk is highlighted as a gap within the current approaches. Similarly, the latter study provides an overview of multi-risk assessment approaches to support adaptation to climate change in mountain regions. The works of Gallina et al. (2016) and Terzi et al. (2019) can help in addressing the challenge of climate change in the light of multi-hazard events. This would significantly help stakeholders, (re)insurance companies, emergency managers and governments in tackling the climate issue.

\subsubsection{Multi-hazard and risk assessments}

The studies that follow in this section describe a diverse set of multi-hazard and risk assessments. They consider several geographical and environmental areas, namely specific countries, coastal and volcanic 
areas, cities and continent-scale assessments. They also review empirical metrics, physical mechanisms, social and economic impacts of multi-hazards, by taking examples from different countries and eventually concentrating the focus on the United Kingdom (UK).

During the past decade, there was a large focus on multi-hazard risk assessments which, as per definition, consider the exposure, vulnerability and multi-hazard interactions to define risk. They have been performed nationally as in the case of China (Zhou et al., 2015), where five major hazards were evaluated (earthquakes, floods, droughts, low temperatures/snow and gale/hail). Or for a single region (Liu et al., 2017), where a specific model of interacting hazards, based on a Bayesian network, was developed in order to calculate the expected multi-hazard occurrences and losses in terms of impacts on society, environment and economy.

Multi-hazard assessments have also been undertaken for coastal areas, which contain large concentrations of people and infrastructure that are exposed to natural hazards such as tsunamis, storm surges and tropical cyclones. Rosendahl Appelquist and Halsnæs (2015) present a global analysis based on the so called Coastal Hazard Wheel (CHW) system and by considering the impact of climate change and hazards such as ecosystem disruption, gradual inundation, salt water intrusion, erosion and flooding. Regional coastal studies have been undertaken, for example in Goa, India (Kunte et al., 2014) or the Ganges deltaic coast of Bangladesh (Ashraful Islam et al., 2016), where a coastal vulnerability index (CVI) was developed with the aid of geospatial techniques (i.e. remote sensing and GIS). The latter also applied a multi-hazard vulnerability assessment in the southeast coast of India (Mahendra et al., 2011), one of the most impacted by the 2004 Indian Ocean tsunami. Such studies prove the utility and associated applicability of empirical metrics, which are able to capture diverse characteristics of multi-hazards and that can eventually benefit the overall resilience and disaster risk reduction policies implemented by local and regional policy makers.

The types of natural hazards are numerous and not all of them are strictly connected to the hydrological cycle or to large-scale atmospheric configurations (see Table 2.5). As an example, volcanically active areas also provided interest with respect to multi-hazard risk assessments. For instance, assessments were performed for Mount Cameroon in Africa (Thierry et al., 2008) and El Misti in Peru (Sandri et al., 2014). In these areas, hazards such as volcanic eruptions (e.g. pyroclastic density currents, lava flows, lahars, tephra fall and ballistic ejecta), landslides, earthquakes pose a significant threat to populations living nearby. 
Multi-risk assessments have also been performed for individual cities. For example, one project evaluated the exposure of Sydney (Australia) to tsunamis, storms and sea level rise through a probabilistic approach (Dall'Osso et al., 2014). A complete risk assessment can be found for two Hong Kong districts (Johnson et al., 2016) and for the city of Conceptión (Chile) (Araya-Muñoz et al., 2017), where in the latter a methodology based on fuzzy logic modelling was developed. Also, a further and more complex three-hazard scenario (storms, floods and earthquakes) was considered for the city of Cologne (Germany), where a multi-risk assessment was applied to predict direct economic losses to buildings and their contents (Grünthal et al., 2006). Investigating multi-hazards at such local scales proves the transversal characteristic of the topic, which indeed can range from local to continental and even global scales. Multiple natural hazards impacting highly-dense populated cities, via the abovementioned hazards of for example by a combination of heatwaves and severe air pollution episodes, linked to persistent anticyclonic or blocked atmospheric conditions, can result in significant societal losses. An example of this can be drawn from the 2003 and 2010 summer heatwaves in Europe (Barriopedro et al., 2011; Le Tertre et al., 2006; Stott et al., 2004).

Finally, continental multi-hazard assessment was performed for Europe in the light of climate change (Forzieri et al., 2016). The investigation considers an ensemble of General Circulation Model-Regional Climate Model (GCM-RCM) climate projections, under the A1B emission scenario, to deliver changes in the frequency of multiple natural hazards, such as heat and cold waves, river and coastal flooding, droughts, wildfires and windstorms. Time periods considered were the historical (1980-2010) and the three future periods up to 2100, namely 2020s (2011-2040), 2050s (2041-2070) and 2080s (20712100). Here, several metrics able to quantify the physical impacts of each hazards, were developed and applied, as for example the Heat Wave Magnitude Index daily (HWMId) (Russo et al., 2015). Moreover, the return periods, along with the exposure (defined as the Expected Annual Fraction Exposed (EAFE)) associated with the natural hazards were also computed. And lastly, a new multihazards metric, that quantifies the annual exposure from all the hazards combined, was also introduced as the Overall Exposure Index (OEI). Forzieri et al. (2016) prove that multi-hazard metrics can be useful for quantifying future climate change impacts at the continental scale.

Within the UK recent studies have started to apply multi-hazards concepts. For instance, weatherdriven hazards such as floods, droughts, windstorms and shrink-swell subsidence were found to interact physically, leading also to compound economic damages (Collet et al., 2018; De Luca et al., 2017; Hillier et al., 2015; Visser-Quinn et al., 2019). Multi-basin (i.e. widespread) floods in GB are shown to be driven by ETCs - the latter identified through Very Severe Gales (VSGs), cyclonic LWTs 
and atmospheric rivers (ARs) (De Luca et al., 2017). This is consistent with ARs contributing to the 10 largest winter flood events in four GB basins considered independently from each other (i.e. singlebasin floods) (Lavers et al., 2013, 2011). Shrink-swell episodes occur between very wet and very dry hydrological periods and thus they are the result of a combination of hazards. These events were identified from subsidence insurance claims within the 1987-2008 period and were also linked to historical climate data in south-eastern England (Harrison et al., 2012). Findings show that for precipitation above $394 \mathrm{~mm}$ within a given past 2-year period, insurance claims were lower in frequency, whereas for precipitation lower than $350 \mathrm{~mm}$ the incidence was higher. Interacting UK hydro-hazards, defined as floods and droughts, were also quantified by considering their magnitude, frequency and duration in both model observations and future projections, making possible the identification of hydro-hazard hotspots (Collet et al., 2018; Visser-Quinn et al., 2019). Indeed, Collet et al. (2018) found that future hotspots are likely to develop along the western coast of England and Wales and over north-eastern Scotland during, winter and autumn respectively for floods and droughts. Similarly, Visser-Quinn et al. (2019) showed that spatio-temporal compound hydro-hazards hotspots lie in north-eastern Scotland and south-western UK. These findings are therefore likely to be relevant for water management companies, with related socio-economic implications. Starting from these two national-scale studies on concurrent floods and droughts events one can also expand the proposed analyses on a larger geographical scale, such as Europe or even the entire globe. This could be achieved by making use of global observational datasets, such as the Palmer Drought Severity Index (PDSI) (Dai et al., 2004; Palmer, 1965), along with future climate projections (e.g. Eyring et al., 2016; Taylor et al., 2011). The resulting findings may therefore help hedging losses by stakeholders and (re)insurance companies with global assets invested for example in hydropower ( $\mathrm{Ng}$ et al., 2017; Turner et al., 2017) and crops production (Leng and Hall, 2019; Zampieri et al., 2017).

\subsubsection{Storm-driven floods}

Flooding events in the UK are known to cause severe impacts in terms of economic and social damages (CCRA, 2016). These events are naturally associated with the passage of ETCs impacting the BI during late autumn and winter seasons (De Luca et al., 2017). Hence, these episodes are a combination of both hydrological and atmospheric processes and can be defined as concurrent hazards, that are also possibly affected by anthropogenic subsurface and surface processes (Gill and Malamud, 2017, 2014; Kappes et al., 2012a). 
In this section, recent examples of exceptional UK storm-driven widespread flooding occurred in the $21_{\text {st }}$ century are summarised. This will provide a relevant review of studies connected to research Chapter 3 (De Luca et al., 2017), by also highlighting the lack of research conducted with respect to the quantification of concurrent flood-wind hazards.

Autumn and winter 2000-2001 floods. During this period, the UK experienced the most severe floods since 1947, during which catchments remained saturated for long periods and severe groundwater flooding affected southern England (Marsh and Dale, 2002). The widespread flooding was found to be one of the most extreme episodes in GB within the 1975-2014 period (De Luca et al., 2017). Autumn 2000 was the wettest in England and Wales since records began in 1766 and the flooding episodes damaged $\sim 10,000$ properties, caused service disruption and $£ 1.3$ billion of economic losses in England and Wales (Pall et al., 2011). The physical mechanisms driving the flooding episodes were identified as westerly circulation patterns and in the passage of diverse frontal systems (Marsh and Dale, 2002) and ARs (De Luca et al., 2017). Some of the storms were remnants of Atlantic hurricanes, which brought heavy rainfall that eventually saturated soils, leading to enhanced runoff and flooding. Seasurface temperatures (SSTs) also played a role, as the thermal gradient can influence storm activity over Europe. During autumn 2000, the SST anomaly was very high $\left(2{ }^{\circ} \mathrm{C}\right.$ degrees above the monthly average) which resulted in a sharp south-west to north-east gradient (Marsh and Dale, 2002). This widespread flooding in England and Wales was also attributed to anthropogenic greenhouse gas emissions (Pall et al., 2011). Thousands of climate model simulations of autumn 2000 weather were performed under various greenhouse gas emissions pathways and then integrated into a precipitationrunoff model for England and Wales. The results showed that in 9 out of 10 cases, greenhouse gas emissions had increased the likelihood of the autumn 2000 floods by more than $20 \%$ and in two runs by more than $90 \%$ (Pall et al., 2011).

Summer 2007 floods in England and Wales. During May-July extreme rainfall hit England and Wales with no precedent since 1847 and some areas reported flooding more severe than that in 1947. In total, 14 people died, thousands experienced misery as a result of properties being flooded (over 55,000 homes and 6,000 businesses) and insurance claims reached $£ 3$ billion pounds (Marsh and Hannaford, 2007). Flash flooding and floodplain inundation were observed from mid-June to the end of July and the dominant flood-generating mechanisms were three slow-moving low-pressure systems (i.e. storms) that caused the exceptional precipitation totals. Soil moisture conditions also played a pivotal role in increasing the likelihood of flooding. Normally, during summer, when there are higher 
temperatures and evaporation, a Soil Moisture Deficit (smd) reduces the likelihood of flooding. By the end of April 2007, soils in England and Wales were their driest since 1961 but this situation reversed completely between May and July, leading to widespread flooding (Marsh and Hannaford, 2007). Similarly, groundwater levels also contributed to fluvial flooding - in late April 2007 levels were low across England and Wales, but they increased sharply between the May-July period. Although robust data on summer storms' frequency is lacking, links to climate change can still be made, because lowpressure systems as the ones observed in 2007 have characteristics that belong to the future climate regime (Marsh and Hannaford, 2007).

Summer 2012 flooding in England and Wales. A similar, but even more exceptional situation led to flooding in summer 2012. In March 2012, the UK experienced one of the most significant droughts in a century, which depressed river flows and groundwater levels (Kendon et al., 2013). Then April to July 2012 were the wettest ever recorded in England and Wales within 250 years, which reversed the attention from drought to flooding. Synoptic conditions driving the change were identified as lowpressure systems affecting the northern UK between 2011 and early 2012, leading to water scarcity in southern GB. From April 2012, the jet stream was more southerly, bringing low-pressure and heavy rains across the country (Parry et al., 2013). April 2012 was the wettest in the UK since 1910 and the wettest for England and Wales within a 250-year period. The causes of the reversed conditions (i.e. drought to flood) reflect the variability of the UK climate. However, robust attributions have not yet been formulated but they could possibly lie with El Niño Southern Oscillation (ENSO) variability and global warming (Parry et al., 2013).

Winter of 2013-2014 floods. Several studies have investigated this exceptional winter period for the UK, which experienced extreme rainfall, fluvial, pluvial, groundwater and coastal flooding due to the passage of many low-pressure systems (Huntingford et al., 2014; Kendon, 2015; Kendon and McCarthy, 2015; Knight et al., 2017; Matthews et al., 2014; Muchan et al., 2015; Priestley et al., 2017; Schaller et al., 2016). In total, 7,000 properties experienced flooding (Muchan et al., 2015) and 18,700 flood insurance claims were made, resulting in $£ 451$ million losses in southern England (Schaller et al., 2016). This winter was the wettest on record since 1910 and the stormiest ever for the UK and Ireland (Matthews et al., 2014). Various physical explanations have been offered. A strong and persistent cyclonic atmospheric circulation over the North Eastern Atlantic Ocean, connected to a very intense North Atlantic jet stream bringing heavy rainfall and wind storms (Knight et al., 2017). Tropical regions have likely influenced the development of the severe extra-tropical circulation and the stratospheric polar vortex, which in turn was associated with a strong westerly phase of Quasi- 
Biennial Oscillation (QBO), which increased the extreme weather conditions (Huntingford et al., 2014; Knight et al., 2017). Other possible causes were the positive phase of the North Atlantic Oscillation (NAO), during the 2013-2014 winter, which is known to drive extreme cyclones that eventually bring high-speed winds and rainfall, leading to flooding in northern Europe (e.g. Hannaford and Marsh, 2008; Pinto et al., 2009). Other possible drivers have been identified including Arctic sea ice extent and solar activity (Huntingford et al., 2014), although these causes may be less certain as the physical mechanisms involved are not yet fully understood.

Winter 2015-2016 flooding in the UK. This episode represented another record-breaking period in terms of weather extremes, with widespread flooding driven by diverse storms impacting the UK (Barker et al., 2016; Burt, 2016; Burt et al., 2016; Burt and Kendon, 2016; McCarthy et al., 2016). Around 16,000 properties flooded in England during December, causing negative impacts on homes, businesses, industry, transport and agriculture. Nine storms (or ETCs) were the cause of the widespread flooding, with storm Desmond being the most powerful and impacting the northwest of England and southern Scotland, with significant damages reported in Cumbria (Barker et al., 2016; Burt et al., 2016; McCarthy et al., 2016). Monthly rainfall records were broken during winter 2015 in the UK, with no precedent since records began in 1910 and quantities up to four times the normal in western and northern areas (Wilby and Barker, 2016). December 2015 was also characterised by exceptionally mild temperatures, which meant that high altitude precipitation fell as rain instead of snow, contributing to more rapid runoff from headwaters. Possible explanations for this remarkable winter are the 2015 El Niño event in the Pacific Ocean connected with a positive phase of the NAO. Also human influence, in terms of global warming, may have contributed to the extreme winter precipitation for the UK with circulation patterns similar to winter 2013-2014 (McCarthy et al., 2016).

The above examples of recent widespread flooding episodes give vital insights into the stormdominating mechanisms that are driving such perils. However, apart from the work presented here in Chapter 3 (De Luca et al., 2017), previous studies do not explicitly quantify links between widespread flooding and ETCs. Hence, although clear and thorough physical explanations about the role of the atmosphere in driving such flooding were made, there is a lack of, for example, a record of widespread flooding events linked with ETCs. As proved by the studies cited above, ETCs, i.e. synoptic (or largescale) low-pressure weather systems occurring in the midlatitudes especially during the winter season, create highly-favourable conditions for flooding in the UK. Apart from severe gales, possibly resulting in property damages and storm surges in coastal areas, they can also bring prolonged and high-intensity rainfall, with associated river flooding (De Luca et al., 2017; Lavers et al., 2011). The UK is situated 
beneath the North Atlantic storm track and represents (along with Ireland) the first country impacted by the passage of ETCs (Matthews et al., 2016b), that can subsequently affect central Europe (Donat M. G., Leckebusch G. C., Pinto J. G., 2010; Priestley et al., 2017). Since ETCs can continue to strengthen after landfall, their impacts may extend to a much larger physical and financial scale than the UK alone (Donat M. G., Leckebusch G. C., Pinto J. G., 2010).

\subsection{Weather patterns}

In this section the literature review's focus changes to weather patterns and their links with hydroclimatic variables and natural hazards over the BI and other regions mostly spread across the European continent. This review has the intent to introduce Chapter 4, which focusses on a quantitative and qualitative analysis of multi-hazards derived from weather patterns in the BI (De Luca et al., 2019a). Thus, the geographical domain expands from catchment/national to regional scale.

Daily atmospheric pressure patterns for the British Isles have been categorised according to the system of LWTs (Lamb, 1972). This classification was originally subjective, meaning that daily weather patterns were assigned manually after inspection of weather charts. A few years after the first subjective classification of LWTs (Lamb, 1972), an objective method to detect daily atmospheric circulation following LWTs was developed (Jenkinson and Collison, 1977). Eventually, both the subjective and objective approach were compared (Jones et al., 1993) and objective LWTs were also derived from reanalyses products (Jones et al., 2013). The main novelty of the objective classification scheme was that it uses grid-point daily mean sea-level-pressure (SLP) analysis for a fixed observation time (such as 00:00 or 12:00 UTC) (Jones et al., 2014). Both the subjective and objective classification schemes yield 27 LWTs comprised of two synoptic (A and C), five purely directional (W, NW, E, N, and S), 19 hybrid combinations of synoptic and pure directional (e.g. CNW, CSE and AE), and one unclassified (U) type (Jenkinson and Collison, 1977; Jones et al., 1993). A current unsolved difference between the objective and subjective schemes is that the former does not show a reduction in the daily W-type count since the 1920s, whereas such a feature was evident in several of Lamb's works (Jones et al., 2014).

For the seven main LWTs, acronyms correspond to the following:

$\mathrm{A}=$ anticyclonic 


$$
\begin{aligned}
& \mathrm{C}=\text { cyclonic } \\
& \mathrm{W}=\text { westerly } \\
& \mathrm{NW}=\text { north-westerly } \\
& \mathrm{E}=\text { easterly } \\
& \mathrm{N}=\text { northerly } \\
& \mathrm{S}=\text { southerly }
\end{aligned}
$$

Over time, as shown below, LWTs attracted much interest. Firstly, because of their strong association (or significant correlations) with other meteorological variables, such as temperature and precipitation (Jones et al., 2014). Secondly, because the objective classification method can be applied not only to the BI but also to other regions of interest within the mid-to-high latitudes (Jones et al., 2013). Thus, a large body of literature addresses LWTs in the UK, Ireland, Sweden, Netherlands, Poland, Iberian peninsula, Spain, Portugal, Mediterranean region, Greece, Turkey, Morocco, Arabian peninsula, China and more broadly across the European continent. These diverse studies are reviewed below for each geographical domain, so that a comprehensive narrative on the diverse uses of LWTs is provided. This will also confirm the vast applicability of the objective LWTs method, not only with respect to different geographical areas, but also to a diverse set of hydroclimatic and atmospheric variables.

For the BI, LWTs were linked to extreme precipitation totals in the 1870s driven by cyclonic weather patterns (Burt et al., 2015). During these heavy rainfall events, a low frequency of anticyclonic and westerly types were also recorded, with the latter contributing to significant rainfall totals mainly in the uplands and north-western coastal regions. Another study looked at relationships between weather patterns and the NAO and the oxygen isotopic composition of rainfall, which is fundamental for paleoclimate reconstruction (Tyler et al., 2016). They found an association between the oxygen isotope and LWTs, especially with the cyclonic types. Other work examined the climatology of cyclones during the period 1871 to 2012 (Matthews et al., 2016b). They found that such weather types drive seasonal precipitation totals, exhibit interannual and multi-decadal variability, exhibit no increasing trend in frequency and storminess, although cyclone intensity has increased especially during the winter season (Matthews et al., 2016b).

LWTs have also been used to downscale GCMs outputs, such as monthly means, persistence and interannual variability of rainfall (Conway and Jones, 1998). Other research has linked ground-level ozone concentrations to variations in atmospheric circulation (O'Hare and Wilby, 1995). Peak ozone concentrations are observed during anticyclonic and easterly days, whereas westerly and cyclonic 
LWTs increase the mean ozone concentration at remote sites due to turbulent mixing processes. In one of the first studies of its kind, Hulme et al. (1993) used LWTs to validate two GCM control simulations against their relative observations of weather type, finding that both GCMs produced too many cyclonic types during winter and that summer temperature variability over the region is not driven by changes in weather patterns. Similarly, a recent study of future LWTs under Representative Concentration Pathway (RCP) 8.5 showed that westerly advection may become more frequent by the end of the 21 st-century, whereas southerly and northerly weather types are projected to reduce in frequency (Stryhal and Huth, 2018).

Numerous other studies focus on the smaller domain of the UK (e.g. Blenkinsop et al., 2015; Burt and Ferranti, 2012; De Luca et al., 2017; Fowler et al., 2000; Fowler and Kilsby, 2002b, 2002a; Jones et al., 2016; Neal and Phillips, 2011; Pattison and Lane, 2012; Pope et al., 2015, 2014, 2016; Richardson et al., 2018; Wetterhall et al., 2012; Wilby, 1998, 1995, 1994, 1993; Wilby et al., 1997; Zhang et al., 2014), with a particular emphasis on precipitation. For example, south-westerly and easterly LWTs are found to yield respectively the largest and smallest amount of daily rainfall in Cumbria (Neal and Phillips, 2011). Another study relates low-frequency/high-magnitude rainfall events in central and southern England to LWTs and found three distinct weather-type clusters (i.e. cyclonic, directional and anticyclonic) that could eventually be used to construct a simple weather model (Wilby, 1998). Moreover, long rainfall records across the north of England show that rising winter rainfall is due to an increase in westerly weather types (Burt and Ferranti, 2012; Fowler and Kilsby, 2002a), whereas declining summer precipitation is linked to a reduction of cyclonic days (Burt and Ferranti, 2012) as well as westerly LWTs over the Yorkshire region (Fowler and Kilsby, 2002a). LWTs were also linked to two sites in central and southern England, then simulation by matrices of transition probabilities along with series of daily and annual precipitation totals, resulted in better estimation of the latter when compared to a simple rainfall generator model (Wilby, 1995). A similar approach, by the same author, was also used to build a stochastic model generator of LWTs and rainfall, which reproduces the different temporal resolutions of rainfall totals (i.e. daily, monthly and annual), with intended applications to climate change impact assessments (Wilby, 1994).

Other UK studies have linked weather types with air quality and pollution (Pope et al., 2016; 2015; 2014; Zhang et al., 2014). For example, Pope et al. (2015; 2014) examined weather patterns and the tropospheric $\mathrm{NO}_{2}$ column. They found that cyclonic conditions show higher seasonality compared with anticyclonic weather patterns, with the former leading to $\mathrm{NO}_{2}$ reduction compared to the latter, during which its accumulation is enhanced. Moreover, LWTs were also associated with surface ozone, that 
can lead to health problems, and results show that anticyclonic and easterly LWTs enhance ozone concentrations over the UK, whereas cyclonic and westerly weather patterns lower ozone, due to advection and replacement with clean air from the North Atlantic (Pope et al., 2016). LWTs have also linked to multi-basin flooding events in GB, with cyclonic and westerly types driving such episodes (De Luca et al., 2017). Another analysis based on a single river basin, showed that extreme floods were generated by cyclonic and westerly types over the 1976-2007 period (Pattison and Lane, 2012). Finally, LWTs have been applied to drought analysis in Yorkshire (Fowler and Kilsby, 2002b) and used to benchmark a new set of weather patterns developed by the Met Office through a national-scale drought analysis (Richardson et al., 2018).

In Sweden, much LWTs research has focussed on air quality assessments (Grundström et al., 2015a, 2015b; Pleijel et al., 2016; Tang et al., 2009). For instance, in Gothenburg air pollutants such as $\mathrm{NO}_{2}$, $\mathrm{CO}, \mathrm{PM}_{10}$ and $\mathrm{O}_{3}$ along with deposition of nitrate, ammonium and sulphate were correlated with annual LWTs frequencies and results show statistically significant values, proving the existence of robust links between LWTs and pollutants (Pleijel et al., 2016). Another study in Gothenburg examined the connections between LWTs and the particle number concentrations (PNC), $\mathrm{NO}_{x}, \mathrm{NO}_{2}$ and $\mathrm{PM}_{10}$, concluding that the anticyclonic, north-westerly, northerly and north-easterly weather patterns are the high-risk categories with higher concentrations of pollutants (Grundström et al., 2015a). Moreover, partially in accord with the above findings, in southern Sweden it was found that ozone levels are higher during anticyclonic, south-eastern/eastern and south-western/southern weather patterns (Tang et al., 2009) and $\mathrm{NO}_{2}$ concentrations higher during anticyclonic, north-westerly and northerly LWTs (Grundström et al., 2015b). Lower $\mathrm{NO}_{2}$ levels were found during easterly, southerly, south-westerly and westerly types (Grundström et al., 2015b).

A national-scale study of precipitation events in Sweden found that cyclonic LWTs accounted for $\sim 70 \%$ of extreme events but only $\sim 45 \%$ for non-extreme ones, with lower westerly and stronger southerly winds favouring extremes (Hellström, 2005). Observed temperatures across southwestern Sweden during winter were also related to LWTs during January (Chen, 2000). The work showed that westerly weather types favour positive temperature anomalies and south-westerly patterns favour positive extremes (i.e. higher temperatures), whereas anticyclonic conditions are related with negative and extreme negative temperature anomalies. This anomaly and extreme temperature patterns are expected for a winter month such as January, as for example anticyclonic conditions bring clear skies with also the possibility of blocking events, that during winter eventually result in severe temperature drops due to the lack of thermal radiation being trapped by clouds. On the other hand, during summer 
anticyclonic weather (and hence blocking) is associated with extreme heat events, possibly leading to concurrent heatwaves, droughts and air pollution episodes.

Within the Iberian Peninsula several studies have applied LWTs to precipitation (Cortesi et al., 2014, 2013; Domínguez-Castro et al., 2015; Paredes et al., 2006). For example, Cortesi et al. (2014) and Paredes et al. (2006) clearly show that much of the rainfall falling into the Iberian peninsula is driven by few LWTs, with the westerly, south-westerly and cyclonic being the most predominant ones. Moreover, the links between weather types and rainfall seem to be stronger during winter and in the western areas of the peninsula. On the Mediterranean coast precipitation is driven by easterly types, whereas in the Cantabrian coast northerly and north-westerly patterns dominate and cyclonic LWTs although result to be the less frequent are the most efficient in generating precipitation (Cortesi et al., 2014). Paredes et al. (2006) looked at the decline in rainfall during March and showed that cyclonic, westerly and south-westerly LWTs are declining in frequency and are accompanied by an increase in anticyclonic pattern, proving that over time low pressure systems are becoming less frequent. LWTs were also successfully used as predictor variables by Cortesi et al. (2013) and Ramos et al. (2010) for modelling national-scale monthly precipitation across the peninsula and linked with modes of climate variability in the northwest. Ramos et al. (2010) also show that the NAO is highly correlated with cyclonic and anticyclonic weather types, the East Atlantic (EA) pattern with the south-westerly LWT, the Eurasian pattern 1 (EA/WR) with westerly and north-westerly types and the Scandinavian (SCA) pattern shows negative correlation with anticyclonic and positive correlation with cyclonic circulation types. Lorenzo et al. (2011) looked at $21_{\mathrm{st}}$-century changes in atmospheric circulation in the northwestern Iberian Peninsula and projected a decrease in the frequency of cyclonic, westerly and southwesterly LWTs during spring, summer and autumn, but an increase in anticyclonic patterns in autumn. Peña-Angulo et al. (2016) also explained with LWTs observed monthly mean maximum and minimum temperatures over the 1950-2010 period, showing that northerly (southerly) weather patterns are linked with a decrease (increase) in temperatures over the region; advection by westerly and easterly types lowers temperatures across coasts but increase them in the central-western areas.

Within Spain there exist a few LWTs studies focusing on the northwest in Galicia (Eiras-Barca et al., 2018; Lorenzo et al., 2008), Leon (Fernández-González et al., 2012; Fernandez-Raga et al., 2017) and the southeast (Goodess and Palutikof, 1998). In Galicia, Lorenzo et al. (2008) found that the anticyclonic pattern is the most frequent across the whole year, with westerly and south-westerly types being significant during autumn and winter. They also showed that the positive NAO phase is correlated negatively (positively) with cyclonic (anticyclonic) LWTs during winter. The East Atlantic 
(EA) pattern also has significant correlations in other seasons with westerly, south-westerly, cyclonic and anticyclonic LWTs and the Northern Hemisphere Annular Modes (NAM) shows correlations with cyclonic and anticyclonic weather. Moreover, Eiras-Barca et al. (2018) showed that winter floods driven by ARs in Galicia are associated with cyclonic weather types, especially with westerly and south-westerly flows. In Leon, Fernández-González et al. (2012) found that during winter (1948-2009) an increase in the frequency of anticyclonic days is observed, with a positive NAO being the main cause of such decline of wet LWTs, that eventually lead to reduced rainfall amounts. These findings led to the development of a downscaling model for precipitation. Remaining in Leon, Fernandez-Raga et al. (2017) investigated the characteristics of raindrops based on the associated atmospheric circulation types and found that during days of westerly and south-westerly weather patterns, raindrops follow a gamma distribution with higher mode. Lastly, a downscaling method for climate impact assessment, which uses weather patterns, was developed and successfully tested in a river basin in southeast Spain (Goodess and Palutikof, 1998).

Across Europe, LWTs have been applied in various ways such as for future climate projections (Demuzere et al., 2009; Donat et al., 2010; Otero et al., 2018; Stryhal and Huth, 2018), storms (Donat M. G., Leckebusch G. C., Pinto J. G., 2010; Donat et al., 2010), precipitation (Ludwig et al., 2016; Plavcová et al., 2014), temperature (Huth, 2010; Otero et al., 2018), air quality (Demuzere and van Lipzig, 2010; Jones and Davies, 2000) and drought (Fleig et al., 2010) analyses. Future projections of weather types made by Otero et al., (2018) suggest an increase in anticyclonic days over southern Europe in all seasons except summer, whereas westerly types increase over north and central Europe especially in winter. Similar studies show an increase in the frequency and persistence of anticyclonic types, an increase in the westerlies, a decrease in the easterly flows and a reduction of cyclonic types (Demuzere et al., 2009; Donat et al., 2010; Stryhal and Huth, 2018). Donat et al. (2010a) performed a study on storms impacting central Europe and showed that about $80 \%$ of such events are connected with westerly weather types and a positive NAO phase. Still in central Europe, Plavcová et al. (2014) demonstrated that high precipitation totals are associated with cyclonic, westerly and north-easterly LWTs and that links between rainfall and atmospheric circulation are stronger in upland areas.

These studies provide useful insights into weather types research over the BI and beyond. Many link LWTs with atmospheric variables and perils that can have significant negative impacts on society and economy, due to heavy rainfall, storminess, air pollutants, floods and drought. In particular, Stryhal and Huth (2018) used an ensemble of reanalyses and GCMs to calculate winter weather pattern frequency, persistence and intensity in central Europe and over the BI. This research could be extended 
by computing weather pattern indices for all seasons and for more RCPs. Furthermore, the weather types derived from the methods could have been directly linked to weather and climate extremes, with an emphasis on multi-hazards events. Similarly, the future weather types projections of Otero et al. (2018) could have covered the entire 21 st-century period, divided for example by three 30 -year periods, instead of only the 2081-2100, along with one or more RCPs. Lastly, since there was a significant focus on the links between weather types and maximum temperatures, this work could be extended to link extreme heat (i.e. heatwaves) with drought and/or poor air quality events.

\subsection{Hydrological extremes and modes of climate variability}

\subsubsection{Wet and dry hydrological extremes}

In this Section the literature review's focus addresses two natural hazards, opposite in nature but that contribute significantly to creating socio-economic damages, from local to global scales, namely wet and dry hydrological extremes. Such natural hazards, although happening during different hydrometeorological conditions, can interact or co-occur both spatially and temporally over a sufficient large geographical domain (De Luca et al., 2019c). Hence, these multi-hazards events can be driven by diverse large-scale weather patterns, for example drought during anticyclonic weather or flooding during cyclonic circulation.

The literature investigating wet and dry hydrological extremes from local to global scales is abundant (e.g. Di Baldassarre et al., 2017; Pechlivanidis et al., 2017; Wang et al., 2014). On the other hand, studies concerning the spatio-temporal interactions, i.e. the co-occurrence of wet and dry extremes in different regions during the same time-period, between these two phenomena are very limited in number (Kreibich et al., 2019). The following literature review will highlight studies on both wet and dry hydrological extremes at different geographical scales and it is aimed to stress the fact that much of the research did not explicitly considered wet and dry extremes as spatio-temporal interacting processes. Furthermore, before the proposed work in this thesis, no investigation was done with respect to concurrent wet and dry hydrological extremes at the global scale. The review is differentiated first by spatial scales and in the final part it provides information about the use of diverse global indices that can bring insights into wet and dry hydrological extremes.

Within the UK, Parry et al. (2013) were the first to acknowledge the interactions between drought and floods. In the study, a notable transition from drought to floods in England and Wales, during 2012, 
was assessed in the context of several hydroclimatological variables (e.g. sea-level pressure, soil moisture, runoff and groundwater). January-March 2012 was the driest period since 1953 for England and Wales, whereas the following nine months were the wettest in 250 years. The physical mechanisms leading to such an abrupt transition are not easy to discern, as changes in weather patterns and jetstream position are part of natural climate variability in the UK. However, it has been suggested that the Atlantic Multidecadal Oscillation (AMO) (Schlesinger and Ramankutty, 1994) is playing a role in driving wet (dry) summers in northern (southern) Europe (Parry et al., 2013). Secondly, most recently, Collet et al. (2018) strictly investigated how flood and drought hazards (defined as hydro-hazards) within GB may change in the future. They looked at the changes in frequency, magnitude and duration of both floods and droughts, the season when they occur and the relative uncertainties associated with climate model projections. This showed that hot-spots of hazards are likely to develop across the western coasts of England and Wales, and in north-eastern Scotland, during winter and autumn respectively for floods and droughts (Collet et al., 2018). Lastly, Visser-Quinn et al. (2019) proposed an impact and uncertainty framework to assess compound floods and drought hotspots in the UK that could also be applied to other regions. They found that north-eastern Scotland and south-western UK are hydro-hazard hotspots and that the variability associated with the hydrological models accounts for the largest contribution when compared with the one derived from the GCMs used.

The works of Collet et al. (2018) and Visser-Quinn et al. (2019) are valuable for water management, national-scale assessments of (multi)hazards risk, emergency managers (e.g. Environment Agency) and for the (re)insurance sector, as they provide for the first time a national quantification of compound floods and drought events. Nonetheless, these studies could be extended by incorporating synopticscale meteorological analysis. For example, the compound events could be investigated in relation to weather types or the major modes of climate variability known to affect the North Atlantic region. This would bring further insight to the hydroclimatological processes at play during the compound hydro-hazard events. Moreover, the proposed methodology by Collet et al. (2018) and Visser-Quinn et al. (2019) could be extended to a larger geographical scale by applying data sets such as the Palmer Drought Severity Index (PDSI) (Dai et al., 2004; Palmer, 1965) or the Standardized Precipitation Index (SPI) (McKee et al., 1995; 1993).

In China, Yan et al. (2013) proposed a catchment-scale assessment of observed abrupt drought-flood transitions, which were found to be driven by changes in weather patterns and that were the cause of significant socio-economic losses. They also quantified the intensity and duration of drought events and linked them to rainfall intensity. Results showed that the more intense the drought, the less the 
chances for a severe rainstorm; and the longer the drought, the greater the chances to observe severe precipitation events, although these results show sensitivity to sub-catchment locations (Yan et al., 2013). In Germany, RCM simulations coupled with an eco-hydrological model provided evidence of a possible increase in more extreme 50-year (return-period) floods and more frequent 50-year droughts in most of the country, with only the Alpine region showing less frequent droughts (Huang et al., 2015). Furthermore, Oni et al. (2016) focussed on a river catchment in Sweden and made use of extreme dry and wet observations to better constrain future hydrological projections.

Predictions of both floods and drought, as independent events, over a river catchment in north-eastern USA were constrained to the extreme phases of two leading climate indices in the North Atlantic basin, the AMO and the NAO (Barnston and Livezey, 1987; Berton et al., 2017). Yoon et al. (2018), on the other hand, focussed their work in Texas (USA), with an investigation of the future changes in wet and dry extremes linked to ENSO. Their results showed that intense drought and extreme precipitation events are set to increase by the middle of the 21 st century and that ENSO could play a role in strengthening their effects. However, they also found that since drought events are getting more intense, groundwater storage is set to decrease in the long-run, despite the projected increase in extreme precipitation. This could be significant for water management practitioners (Yoon et al., 2018). The work of Dong et al. (2011) in the central USA, focussed on two memorable hydrological years, 2006 and 2007, where lack of rainfall and extreme precipitation, leading to drought followed in time by floods, were respectively recorded. The causes of these wet-dry changes were linked to large-scale atmospheric dynamics, with moisture transport from the Gulf of Mexico playing a role. Similarly, Dirmeyer and Brubaker (1999) investigated the role of moisture transport during another two exceptional dry and wet years for the USA, namely the 1988 and 1993, however they did not consider the hazards as interacting perils.

At a larger scale, Pechlivanidis et al. (2017) investigated simulated wet and dry hydrological extremes in five river basins worldwide. They used GCMs, under four RCPs, coupled with a suite of hydrological models. Results showed that anthropogenic climate change may have a severe impact at the end of the 21 st-century, by increasing both high and low flows. They also stressed that climate impact studies are affected by uncertainty from both climate and impact models used. The work of Pechlivanidis et al. (2017), although it has a global focus, did not specifically address the topic of multi-hazards, as wet and dry hydrological extremes were treated as separate processes, however the methodology used would be highly valuable for assessing future multi-hazards impacts. In Europe, the assessment of changes in both floods and drought under anthropogenic climate change was performed 
using a sub-ensemble of RCMs coupled with three hydrological models (Roudier et al., 2016). This showed a contrast between northern and southern Europe, such that flood intensity is set to increase (decrease) in the south (north) and drought magnitude and duration may increase over the Mediterranean countries, south of the UK and Ireland, although such results are less robust compared to floods. The study deepens understanding of future possible changes in both floods and drought in Europe. However, the two hydrological hazards were treated as separate, non-interacting, processes. Hence, there is scope to evaluate the future total impacted area $\left(\mathrm{km}_{2}\right)$ by the two hydrological hazards and transition times between a flood (drought) and drought (flood) event at the continental-scale.

Other studies investigate wet and dry hydrological extremes using indices such as the PDSI (Briffa et al., 2009; Chen et al., 2017; Dai et al., 2004; Kangas and Brown, 2007; Palmer, 1965; H. Wang et al., 2018; Wang et al., 2014), SPI (Bordi et al., 2009; Domínguez-Castro et al., 2018; García-Valdecasas Ojeda et al., 2017; Kangas and Brown, 2007; Martin, 2018; McKee T.B., Doesken N.J., 1995; McKee et al., 1993; Sun et al., 2016; Tošić and Unkašević, 2014; H. Wang et al., 2018) or Standardized Precipitation Evapotranspiration Index (SPEI) (Chen et al., 2017; Domínguez-Castro et al., 2018; García-Valdecasas Ojeda et al., 2017; Sun et al., 2016; Vicente-Serrano et al., 2010; Wang et al., 2018).

For example, the PDSI was used to evaluate the combined effect of the Pacific Decadal Oscillation (PDO) and ENSO on global wet and dry changes over land, showing that when these two modes of climate variability are in phase (e.g. El Niño-warm PDO) they amplify the wet and dry events (Wang et al., 2014). Others studied the relationship between ecosystem global primary productivity linked to wet and dry conditions, using the PDSI, SPI and SPEI among others (Wang et al., 2018). They found that primary productivity anomalies at the regional scale and on annual and seasonal time-scales are more sensitive to PDSI, whereas they are most correlated with SPI and SPEI, respectively in the northern and southern hemispheres.

The PDSI and SPEI were also used to quantify wet and dry trends in six regions over China, differentiated by Köppen climate zones (Chen et al., 2017; Rubel and Kottek, 2010). They found that for both wet and dry trends these indices agree in five out of six regions and the increasing dryness occurred in the humid and arid transition region of China. At the global scale, the SPI and SPEI were used to explore wet and dry links with ENSO, PDO and the NAO (Sun et al., 2016). The study found that ENSO has the dominant global signature in independent wet and dry changes, followed by the PDO in North America and eastern Russia, and the NAO affecting Europe as well as north Africa. The SPI was also used in a global multi-model ensemble analysis of future projections in pluvial and 
drought events (Martin, 2018). This showed that more severe pluvial events are expected in already wet regions and the same applies for more severe drought conditions in dry areas. They also show that severe pluvial/drought events are increasing in many regions with a drying/wetting trend.

The majority of these studies based on global indices derived from precipitation, temperature and evapotranspiration, are focussed on the observational period and, although they derive extreme wet and extreme dry properties, they do not address multi-hazards by coupling the opposite extremes. Thus, with such investigations one could have also defined explicitly extreme wet and extreme dry events at the global scale, quantifying their concurrent spatio-temporal patterns and any links with modes of climate variability. This could have shed light into flooding and drought events concurrently happening, within a given window of time, in different parts of the globe. Such insights would benefit global (re)insurance companies, commodity brokers, or stakeholders with global portfolios.

\subsubsection{Fluvial flooding and modes of climate variability}

Fluvial flooding events are known to cause significant socio-economic damages (Munich Re, 2017b, 2017a; UNDRR, 2017b) and flood risk, in the future, is expected to increase due to anthropogenic climate change and socio-economic changes (Arnell and Gosling, 2016; Winsemius et al., 2016). Over years to decades, regional and global precipitation patterns are driven by modes of climate variability (or teleconnections). These are recurrent climate patterns that influence specific regions around the world with an oscillatory behaviour, generally represented by positive (+) and negative (-) values. Modes of climate variability, for instance, have influences not only on precipitation (e.g. Dai and Wigley, 2000; Enfield et al., 2001; Hurrell, 1995; Larkin and Harrison, 2005; Ning and Bradley, 2016; Sutton and Hodson, 2005), but also on temperature (e.g. Hurrell, 1995; Larkin and Harrison, 2005; Ning and Bradley, 2016), and storm tracks (e.g. Harding and Snyder, 2015; Wang et al., 2018). Hence, since fluvial flooding is mainly driven by extreme rainfall events, modes of climate variability have a significant effect on this hazard and they could be used to improve prediction and risk models (Lee et al., 2018; Ward et al., 2014b).

At present, there are numerous studies that have investigated relationships between fluvial flooding and modes of climate variability, (e.g. Brandimarte et al., 2011; Emerton et al., 2017; Ezer and Atkinson, 2014; Mallakpour and Villarini, 2016; Nobre et al., 2017; Ward et al., 2014a, 2014b). The dominant modes known to affect regional and global precipitation patterns are ENSO (Trenberth, 1997), PDO (Mantua and Hare, 2002) and AMO (Schlesinger and Ramankutty, 1994). However, other climate indices of regional interest include the NAO (Barnston and Livezey, 1987), the Pacific-North 
American pattern (PNA) (Barnston and Livezey, 1987; Liu et al., 2017) and the QBO (Baldwin et al., 2001).

The ENSO teleconnection is an interannual variation in winds and SSTs over the tropical eastern Pacific Ocean, that affects the climate within the tropics and sub-tropics. The ENSO warm phase is also known as El Niño, whereas the cold phase is known as La Niña. It represents the climate mode with larger spatial impacts on flooding among all the other indices and its effects operate on interannual timescales. During a positive phase of ENSO (or El Niño) flooding tends to occur mostly in the southern United States (USA), parts of central North America, Mexico, central/northern Argentina and Uruguay, central/southern Europe, south-central and eastern Africa, middle east, eastern China, Japan and southern New Zealand (Emerton et al., 2017; Lee et al., 2018; Ward et al., 2010; Ward et al., 2014a). Due to its relatively short time-scale and global impacts, ENSO is the most studied teleconnection among the others. For example, Emerton et al. (2017) used a 20th-century reconstructed river flow dataset (ERA-20CM-R), to investigate how El Niño and La Niña (the negative phase of ENSO) events affect river flooding around the globe. They showed that the dataset is able to capture regions with enhanced risk of flooding during the two ENSO phases, however they also conclude that the likelihood of flood hazard is more complex than is currently reported. This is due to uncertainties within the computed historical probabilities (or datasets used) and discrepancies between results obtained from hydrological analysis and precipitation, as the latter not always coincide. Emerton et al. (2017) provide insights into the spatial patterns of floods and low-flow events with respect to ENSO. However, such events could be linked in isolation or combination with other climate indices, such as the PDO and AMO. Lee et al. (2018) attributed seasonal river peak flows to several climate patterns, such as ENSO, PDO, NAO and AMO, by using both observations and river flow simulations from a global hydrological model, with the aim to create a global, season-ahead prediction model. Ward et al. (2010) looked at the sensitivities of annual mean and flood discharges to ENSO and they also investigated how global precipitation and temperature are affected by the same climate pattern. ENSO has also been used to investigate global flood risk (i.e. impacts on society and economy and not only on flood hazard) (Ward et al., 2014b). This revealed that ENSO-driven floods had significant impacts on the size of affected population, gross domestic product (GDP) and economic damages. The authors conclude that climate patterns, such as ENSO, need to be integrated into disaster risk analyses and policies as there could be the possibility to develop probabilistic flood-risk projections. Ward et al. (2014a) examined sensitivities of simulated annual river floods to ENSO and found that within the period 1958-2000 ENSO significantly affected floods over one third of the global land surface and that this influence is greater than for average river flows. Moreover, they show that the relationship between 
ENSO and floods is non-stationary and stress the importance of including ENSO in flood risk forecasts (Ward et al., 2014a).

Aside from the above global studies of ENSO impacts on river flooding, there are many regional analyses (e.g. Cayan et al., 1999; Hamlet and Lettenmaier, 2007; Kiem et al., 2003; Mallakpour and Villarini, 2016; Munoz et al., 2018; Nobre et al., 2017; Ouyang et al., 2014; Rimbu et al., 2004; Tootle et al., 2005). For example, in Europe, Nobre et al. (2017) focussed on the relationships between ENSO, NAO and the EA pattern (Barnston and Livezey, 1987) with respect to extreme floods, calculated as occurrence and intensity of extreme rainfall and flood occurrence and damage. They show that NAO and EA play a stronger role in controlling extreme rainfall when compared to ENSO, which however still maintains a significant role in some regions. Flood occurrence and damage were also strongly linked with these climate patterns, meaning that they need to be considered when assessing both flood hazard and risk across Europe (Nobre et al., 2017). Furthermore, Tootle et al. (2005) investigated the relationships between ENSO, PDO, AMO and NAO and river flows across the USA and showed that some of these climate patterns may also interact. For instance, the AMO can affect La Niña impacts in the southeast and the NAO La Niña impacts in the Midwest USA. Within the lower Mississippi river basin (USA), flood hazard is shown to be affected by ENSO and AMO which, when combined with river engineering measures (i.e. artificial cut-offs and levees) originally aimed to reduce the hazard, greatly amplified the flood magnitude (Munoz et al., 2018).

Continuing in the USA region, Mallakpour and Villarini (2016) looked at the influences that climate patterns such as ENSO, NAO, PDO, AMO and PNA have on the frequency of flooding over the central USA. They found that climate variability explains observed changes in flood frequency, that each climate mode affects a specific part of the region, and that the PNA plays the most significant role among the other climate patterns. In China, Ouyang et al. (2014) investigated the observed past 100year links of ENSO and PDO with respect to precipitation and river flows. They show that both precipitation and river flows decrease in magnitude during El Niño and warm PDO phase, whereas an increase is observed during La Niña and cool PDO phase, however several differences are observed at regional and seasonal scales. For example, over the Yellow River, Yangtze River and Pearl River basins, precipitation and river flows occurring in October-November are more influenced by both El Niño and La Niña events compared to the Songhua River basin (Ouyang et al., 2014). Such hydroclimatological differences are expected for a large country such as China and they once again highlight the importance of constraining both precipitation and river flow forecasts with modes of climate variability. 
The PDO pattern (Mantua and Hare, 2002) ranges from interannual to interdecadal time scales and it is detected as warm or cool surface waters in the Pacific Ocean, in the region north of $20^{\circ} \mathrm{N}$. During the PDO positive (or warm) phase peak flows are observed in the USA, central and southern South America, Europe and central Asia (Lee et al., 2018). Moreover, when PDO is positive, the central and south-western USA tends to experience flooding during all the seasons, except during winter (Mallakpour and Villarini, 2016; Tootle et al., 2005), when increased streamflow are observed in north-western North America (Hodgkins, 2009; Khaliq and Gachon, 2010; Neal et al., 2002).

As mentioned above, some studies that looked at the interactions between ENSO and river flows, peak flows and flooding, also included signatures from the PDO (Hamlet and Lettenmaier, 2007; Lee et al., 2018; Mallakpour and Villarini, 2016; Ouyang et al., 2014; Tootle et al., 2005). However, other works considered the PDO on its own or along with other indices (Cai and Rensch, 2012; Hodgkins, 2009; Hodgkins et al., 2017; Khaliq and Gachon, 2010; Neal et al., 2002), with North America being the favoured study area. For example, Hamlet and Lettenmaier (2007) looked at changes in flood risk during the 20 th century across the western USA and how they were associated with global warming and climate patterns such as the PDO and ENSO. They found that both indices contributed to changes in flood risk and that their signal is regionally-distributed and the strongest responses occur when PDO and ENSO are in phase. Hodgkins et al. (2017) investigated observed trends in flood occurrence in North America and Europe, and found that the statistically significant trends detected were almost the same as the ones expected by chance, with changes in major floods dominated instead by climate patterns such as the PDO and AMO. In north-western North America the PDO is found to have an effect on winter river flows, with flows being higher during the warm PDO phase (and vice versa) (Khaliq and Gachon, 2010). The PDO is also known to influence flows in Alaska (Hodgkins, 2009; Neal et al., 2002), where its signal does not change significantly on annual river flows, but it does on monthly and seasonal time scales, with warm PDO winter river flows being higher than the cold PDO ones (Neal et al., 2002). Moreover, changes in river flows in Alaska between the cold (1947-1976) and warm (1977-2006) PDO phase in winter, spring, summer and annual maxima (AMAX) varied between glaciated and non-glaciated basins. The former were the most affected or the ones showing the largest changes (Hodgkins, 2009). The PDO is also thought to have contributed to the 2011 southeast Queensland flood in Australia (Cai and Rensch, 2012). 
The AMO exhibits variations over multi-decadal timescales due to changes in SSTs across the North Atlantic. The index is computed with any linear anthropogenic global warming trend (or the effect of green-house gases on SSTs) removed from the analysis. A positive (or warm) AMO phase is typically associated with peak flows and flooding in north-western USA, southern and equatorial Africa, central and eastern Russia, southern Asia and Europe (Hodgkins et al., 2017; Lee et al., 2018). On the other hand, a negative (or cold) AMO phase brings increased streamflow in the upper/middle Mississippi river basin, south-eastern and south-western USA (Tootle et al., 2005). In fact, flooding in the central USA is negatively related with a positive AMO phase during almost all seasons, except for summer (Mallakpour and Villarini, 2016). Lee et al. (2018) also used the AMO as a predictor to build a global season-ahead river peak flows prediction model at the global scale.

Apart from the above global assessment, other studies of AMO influences on river flows, peak flows and flooding, tend to be regional and/or focussed on the USA (e.g. Berton et al., 2017; Hodgkins et al., 2017; Mallakpour and Villarini, 2016; Munoz et al., 2018; Rogers and Coleman, 2003; Toonen et al., 2016; Tootle et al., 2005; Tootle and Piechota, 2006). For example, a USA-wide analysis of river flows did show that in addition to ENSO, other climate patterns, such as AMO, PDO and NAO also influence river flow variability (Tootle et al., 2005; Tootle and Piechota, 2006). Moreover, it is also demonstrated the combined effect of AMO, PDO and NAO with respect to ENSO, with the AMO influencing La Niña impacts in the south-eastern USA (Tootle et al., 2005). Hodgkins et al. (2017) investigated major floods in North America and Europe and found that change in the occurrence of observed floods was dominated by multidecadal variability, with the AMO showing more than three times significant relationships compared to significant long-term trends. In the central USA, the change in flood frequency, when assessed using a Poisson regression model, was attributed to several modes of climate variability, with the AMO playing a significant role (Mallakpour and Villarini, 2016). Over the Mississippi river basin the AMO is also found to have an influence on both flood hazard and river flows (Munoz et al., 2018; Rogers and Coleman, 2003). In the latter case, winter relationships between river flows and AMO, PNA and ENSO are investigated within a Mississippi river basin's network of 554 hydrological gauges and results show that the AMO has a statistically significant influence in the upper Mississippi valley and on low/high winter river flows respectively during 1930-1959 and 1965-1994 (Rogers and Coleman, 2003). A more local study focussed on the Merrimack River basin in north-eastern USA showed that observed river discharges were significantly correlated with both extreme positive and negative phases of the AMO and NAO, thereby providing a basis for near-term prediction of floods and droughts (Berton et al., 2017). 
The above studies contribute to the understanding of hydroclimatological processes at regional to global scales, and may provide useful knowledge for stakeholders, governments, (re)insurance companies and emergency managers. They also strengthen the case for including modes of climate variability into seasonal/sub-seasonal weather and hydrological forecasts. Most of the studies showed the correlations between (extreme) river flows and climate indices and since many of them made use of large numbers of correlation tests (e.g. for continental/global analyses), the chances of incurring Type I errors (i.e. false positives) increase significantly. Thus, the robustness of these analyses would be enhanced by, for example, performing the Bonferroni correction (Bonferroni, 1936; Sedgwick, 2014) on the p-values obtained. Such correction takes into account the total number of correlation tests performed and adjusts the p-values accordingly. This would certainly reduce the overall number of significant correlations, but the results would have been stronger and the non-significant correlation patterns still shown in the maps. Lastly, none of the study computed the hydrological extreme wet correlations along with the extreme dry events in the context of multi-hazards, i.e. concurrent wet and dry hydrological events, and multiple modes of climate variability at the global scale. By doing so, the findings would have contributed in assessing extreme wet and extreme dry hydrological observations and events. This knowledge could be of high interest for stakeholders with global assets invested for example in hydropower (Ng et al., 2017; Turner et al., 2017) crop production (Leng and Hall, 2019; Xie et al., 2018; Zampieri et al., 2017) or transport networks (Koks et al., 2019).

\subsection{Summary}

Here in Chapter 2 a literature review related to the research Chapters 3-5 was presented. First, a general overview about the most common definitions and general applications of multi-hazards is provided, to introduce the reader to the main framework of the work (Section 2.2). Hence, this section related to all research Chapters 3-5. Then, from this general overview the focus changes to interacting flood and wind-storms events in the UK (Section 2.2.1) and therefore this refers directly to Chapter 3, where research on observed multi-basin flooding linked with ETCs in GB is presented (De Luca et al., 2017). Secondly, the literature review addressed the topic of a specific classification of weather patterns (i.e. the LWTs), mostly used over the BI, with connections on hydroclimatic variables and natural hazards over different regions (Section 2.3). This Section provided insights into the use of LWTs and therefore laid down the bases for Chapter 4, where observed and projected persistence of LWTs and associated multi-hazards over the BI is shown (De Luca et al., 2019a). The last part of the review (Section 2.4), provides background relevant for a critical understanding of Chapter 5, that considers the spatio- 
temporal interactions of wet and dry hydrological extremes at the global scale, also linked with modes of climate variability (De Luca et al., 2019c). Thus, Section 2.4.1 focussed on an overview of studies that looked at both wet and dry hydrological extremes. Finally, Section 2.4.2 brought information about river flooding driven by a set of modes of climate variability.

In the following Chapter 3 insights about observations on multi-basin flooding linked with ETCs in GB are given and discussed. Specifically, river floods are quantified by making use of peak river flow AMAX data over a time-window of $~ 16$ days and ETCs through VSGs, cyclonic LWTs and ARs. The results presented can have implications for stakeholders, (re)insurance companies and emergency managers in GB and beyond (De Luca et al., 2017). 


\section{Chapter 3 \\ Extreme multi-basin flooding linked with extra-tropical cyclones}

The following Chapter has been published in the journal Environmental Research Letters and the current form represents the format of the first submission to the journal (see Annex 3 or De Luca et al. (2017) for the final published version). The author of this thesis (PDL) contributed to the development of the research questions, prepared the data, performed the analyses, created the pictures and wrote the first manuscript draft. RW conceived the original idea, JH contributed to the statistical analyses and created Figure 3.6. NQ gathered data from the Scottish Environmental Protection Agency (SEPA) and performed the time to peak modelling. All the authors contributed to the writing of the manuscript.

\subsection{Introduction}

River floods endanger lives, damage the built environment, cause disruption and accrue significant economic losses (Barredo, 2007; Hall et al., 2005; Merz et al., 2010). The Sendai Framework for Disaster Risk Reduction (UNDRR, 2015) recommends better mapping and management in areas prone to flooding to increase resilience through public and private investment in disaster risk prevention and reduction measures. The UK Climate Change Risk Assessment (ASC, 2016) highlighted that flood risks are already significant in the UK and are expected to rise as a consequence of climate change. Pragmatic and well-targeted actions were called for with respect to high magnitude flood risks for communities, businesses and infrastructures (ASC, 2016). Case studies demonstrate that highmagnitude flood episodes, mainly occurring during the late autumn and winter seasons, also tend to impact large areas covering multiple river basins (Barker et al., 2016; Marsh, 2008; Muchan et al., 2015; Parry et al., 2013). Such widespread flooding episodes intuitively can pose higher socioeconomic risks compared to single-basin flooding events.

To date, fluvial flooding has tended to be studied on a basin-by-basin basis with respect to physical processes and impacts (Blöschl et al., 2015; Gaál et al., 2015; Huntingford et al., 2014; Mallakpour and Villarini, 2015; Merz, R. Blöschl, 2003; Merz et al., 2014; Nied et al., 2016; Schaller et al., 2016; Viglione et al., 2010). Statistical methods for creating designed floods rely on pooled data from multiple basins (Cunderlik and Burn, 2003; Kjeldsen and Jones, 2009), but these approaches are 
indifferent to any spatial and temporal relationships in the data. Multivariate extreme value statistics are useful for estimating return periods for major events (Heffernan and Tawn, 2004; Keef et al., 2013, 2009) and for characterizing spatially varying and time-lagged extreme flows (Chen et al., 2012; De Waal, D., Van Gelder, P. and Nel, 2007; Wyncoll and Gouldby, 2013). Within the reinsurance sector, weather-driven multi-basin 'catastrophe models' are widely used to estimate economic losses due to flooding (Qu Y.; Dodov B.; Jain V.; Hautaniemi T., 2010; Sampson et al., 2014). Statistical approaches to joint probabilities (Cameron et al., 1999; Chen et al., 2012; De Waal, D., Van Gelder, P. and Nel, 2007; Ghizzoni et al., 2012, 2010; Lamb, 2006) have been extended to multi-basin flooding (MBF), as well as simulation of extreme flow events for northeast England using conditional probability models (Lamb et al., 2010).

Historical MBF episodes have also been investigated in Germany (Uhlemann et al., 2014, 2010) and across Europe (Berghuijs et al., 2019), where for the latter case climate models have been also used to project economic losses (Jongman et al., 2014). However, so far in Great Britain (GB) and elsewhere, there have been no national-scale analyses using simple and pragmatic statistics that specifically focus on the spatio-temporal characteristics of extreme MBF and their links with extra-tropical cyclones (ETCs), that are known to enhance the impacts of the most extreme single-basin floods (Lavers et al., 2011; Matthews et al., 2018).

The MBF approach proposed in this chapter overcomes the limitations of single-basin return period estimation, with the possibility of developing a national-scale return period for improved risk communication. A MBF episode can simultaneously impact very large regions, with the chance to overwhelm emergency responses, for example coordinated by the UK Environment Agency (EA). In addition, MBF may coincide with ETCs, which together create a multi-peril scenario of flood-wind impacts. Such episodes may be more severe than expected; illustratively, combined flood-wind impacts at the 16-year return period are increased by the link between perils, costing an additional $£ 0.3$ billion for domestic UK properties (Hillier et al., 2015).

The main research questions that this chapter aims to answer are the following:

1) What is the spatio-temporal distribution of MBF episodes?

2) What are the most frequent weather patterns observed during these widespread floods?

3) How are multi-basin floods, atmospheric rivers (ARs) and very severe gales (VSGs) linked? 
This chapter therefore introduces a pragmatic approach for detecting and quantifying the characteristics of extreme MBF episodes and their links with ETCs. It also uses new and established metrics that could be relevant for assessing hydroclimatic impacts (Ekström et al., 2018). GB has been used as a pilot area, but the techniques deployed are applicable wherever there are gauged river flow data. The first research question has been answered by investigating a window of 1 to 19 days for coincident peak flow annual maxima (AMAX) in 261 non-nested river basins during the 1975-2014 period. The reason behind choosing this time-window length is that it provides a sufficient, but not too long, time for ETCs to completely pass through GB and for precipitation to infiltrate into the ground, eventually enhancing runoff and flooding events. Once the MBF episodes have been identified, the most extreme have been plotted in maps and their occurrences counted on a monthly-basis. The second research question has been fulfilled by counting the occurrence of daily Lamb Weather Types (LWTs) during the widespread flooding episodes. Such counts made possible the identification of the most frequent LWTs driving MBF. Then, the third research question is addressed by simply checking if ARs co-occurred during the most extreme MBF episodes and by correlating VSGs with MBF.

The following sections describe the data (Section 3.2), methodological approach and metrics (Section 3.3), then the six most extensive and temporally distinct MBF episodes identified, along with their links with ETCs (Section 3.4). It is confirmed that these most extensive MBF had widespread impacts (Environment Agency, 2003; Hulme, 1997; Kelman, 2001; Marsh and Dale, 2002; Meteorological Office, 1992; Perry, 1980; Watkins and Whyte, 2008) and mostly occurred during winter. A particularly powerful aspect of this approach is that it is compatible with the synoptic-scale (i.e. $\sim 1,000 \mathrm{~km}$ horizontal length scale) of atmospheric conditions and land-surface properties. This allows severe MBF episodes to be evaluated alongside categories of atmospheric circulation (LWTs), antecedent rainfall as a proxy for soil moisture (Standardized Precipitation Index, SPI), ARs, and storminess (VSGs, frequency). Moreover, the hydrological response (joining time, $J t$ ) for large and small basins is examined to determine lagged responses in the system. Finally, the chapter concludes with a discussion of the causes and implications of extreme MBF and their relationship with ETCs (Section 3.5) and a summary of the overall research (Section 3.6).

\subsection{Peak Flow and SPI Data}

Highest instantaneous (15-min) peak flows ( $\left.\mathrm{m}_{3} / \mathrm{s}\right)$ in each water year (1 st October - 30th September) were extracted from the 1975-2014 record. These AMAX series were drawn from 261 spatially 
representative gauged basins across GB, within a 40-year block that provides the best compromise between spatial and temporal coverage, so that most of GB land area could have been analysed for a sufficient longly climatic period. The network of stations is non-nested (i.e. one gauge per basin) and covers $60.1 \%$ of GB land area (Figure 3.1). Non-nested gauging stations, as opposed to nested ones, make it possible to consider each river basin as one individual entity, without compromising the statistical methods by adding a bias towards the real number of basins involved in the multi-basin floods. This is equivalent to Network A used in a previous related study (Wilby and Quinn, 2013) but with more representative coverage across GB. The mean basin area is $482 \mathrm{~km} 2$, ranging from $6.5 \mathrm{~km} 2$ (Allt Leachdach) to $9,948 \mathrm{~km} 2$ (Thames), and average basin elevation is $149 \mathrm{~m}$ a.s.l. Data were obtained from the National River Flow Archive using WINFAP-FEH v4.1: http://nrfa.ceh.ac.uk/winfap-fehfiles and, for Scotland, from the Scottish Environment Protection Agency.

The SPI for timescales 24-, 18-, 12-, 6-, 3- and 1-months were obtained from the Centre for Ecology \& Hydrology (CEH) (https://catalogue.ceh.ac.uk/documents/5e1792a0-ae95-4e77-bccd2fb456112cc1) (Tanguy M., Kral F., Fry M., Svensson C., 2015). In this chapter all the available SPI time-scales were used, so that sensitivity with respect to soil moisture was also quantified. The data cover all GB within the period 1961-2012 with the territory divided into 105 Hydrometric Areas (HAs), each with a specific SPI time series. To remain coherent with the main analysis, the SPI data used range from 1975 to 2014, as with the chosen 40 years of hydrological records. CEH provided the remaining two years (2013-2014) of SPI data coherent with the above dataset. To calculate a multibasin SPI value for an episode, the mean SPI for the HAs impacted is calculated as defined by event set and $L$ (see Section 3.3). Multiple AMAX on a given day are allowed because they are needed to distinguish the HAs impacted and since each time window has been treated independently, there exist no duplicated dates that need to be removed. 


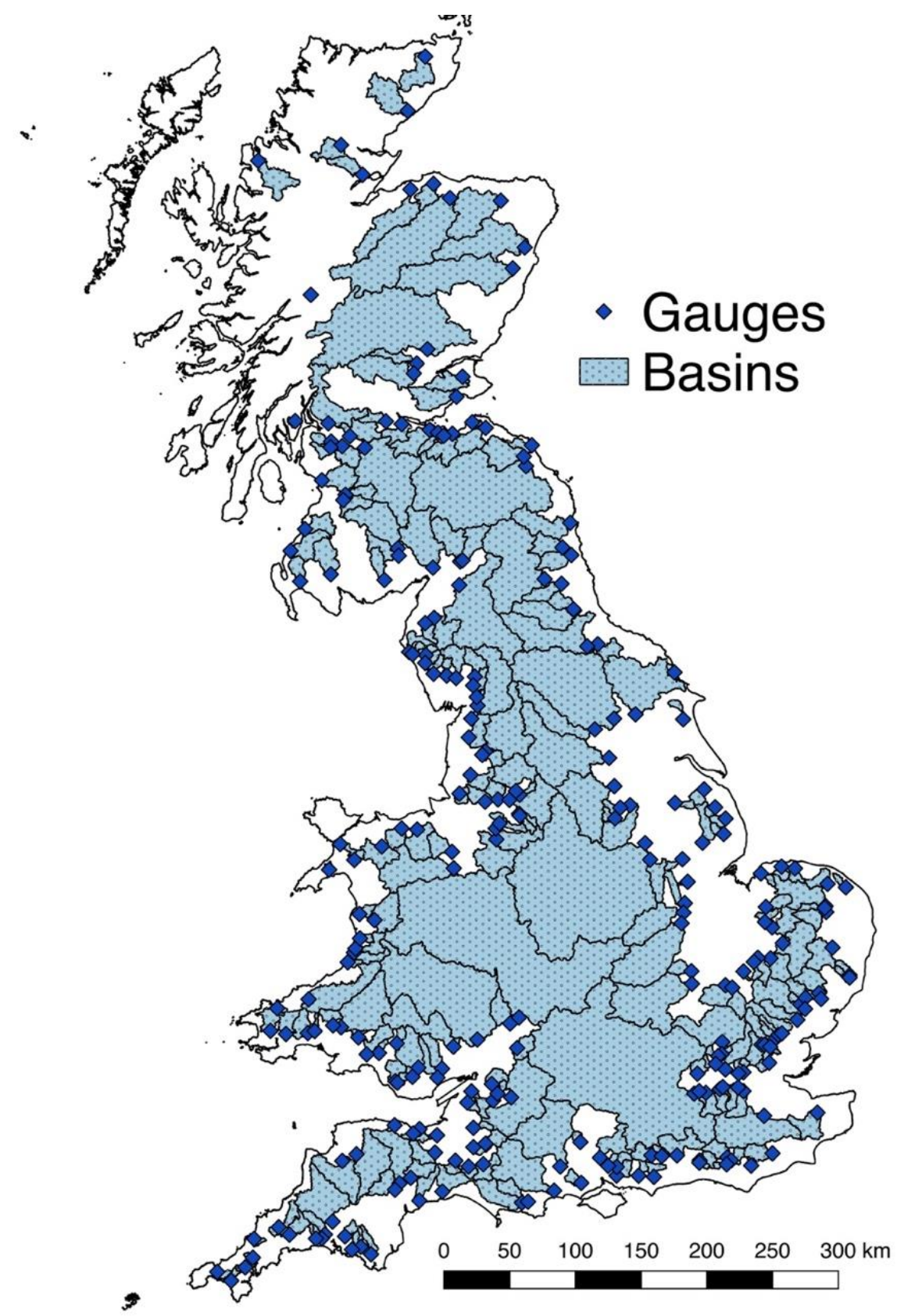

Figure 3.1 Network of hydrological stations and related basin areas used in the analyses. The 261 non-nested gauges were selected from an initial network of 649 (Annex A.1.1 Figure S3.1) based on record length, and geographic coverage. Some areas are under-represented (e.g. east England) because they are either ungauged or do not have data for the 1975-2014 period. 


\subsection{Methods}

\subsubsection{Quantifying multi-basin flooding episodes}

The severity of a single-basin fluvial flow is readily defined by the peak discharge, and it is also possible to index MBF using the peak flow in the most extreme basin (Lamb et al., 2010; Wyncoll and Gouldby, 2013). Alternatively, severity may be defined in terms of economic impact (Hillier et al., 2015), but complete and comparable residential and commercial loss estimates are extremely difficult to obtain for all but the most severe historical events. Recent studies have begun to assess the severity of flooding episodes by considering the whole UK, effectively extending the paradigm applied to single-basin floods by looking at monthly mean river flows (Barker et al., 2016; Huntingford et al., 2014), seasonal river flow accumulations (Muchan et al., 2015) and compound flood-drought events (Collet et al., 2018; Visser-Quinn et al., 2019). However, a pragmatic metric to define the hydrological severity of a multi-basin episode, particularly one that highlights the spatial distribution of basins involved, is not yet in widespread usage.

The MBF metrics proposed in this analysis are based on a deliberately straightforward procedure that counts the total number of basins involved in each episode. The principal metric uses the summed number of independent gauges $n_{\mathrm{g}}$ that report AMAX within a given multi-day time window. This extends a previous single-day approach (Wilby and Quinn, 2013) to include MBF episodes where AMAX flows fall within a window of length $L$ days (up to 19) ending on the day when most gauges report their AMAX, denoted $d_{\max }$.

The following three step procedure was implemented for each time window: (1) determine $n g$ g, for each day $j$ and list these in descending order; (2) for largest $n \mathrm{~g}, \mathrm{j}$, search the list for any dates within the time window being quantified and add $n_{\mathrm{g}, \mathrm{j}}$ values to $d_{\max }$ whilst deleting these days from the list; and (3) repeat the previous step, descending the list until the end. Hence, the most extreme MBF episode is defined as that with the greatest number of basins exhibiting AMAX flows within the specified time window. However, episodes were classified using two other ways to emphasize different aspects of the flood character. These are: i) the multi-basin Flood Yield $(m F Y)$ and; ii) the total drained area (TDA). These use the same list of episodes, and basins, defined by the procedure above, but re-value the quantity used to rank severity. $m F Y$ takes Flood Yields $(F Y=Q / A$ m3s-1km-2, where $Q$ is peak flow AMAX m3s-1 and $A$ is basin area $\mathrm{km}_{2}$ ) calculated for individual basins and then sums them. The $m F Y$ index is potentially biased towards small basins, whereas $T D A$ with regard to larger basins. 
Within the following analyses, four different event sets (A-D) have been created and used as a sensitivity test to improve the robustness of the findings related to the most extreme MBF (event set E). Such event sets consider both single-basin floods and MBF, with the latter defined by making use of different severity metrics just defined in the text, i.e. $n_{\mathrm{g}}, m F Y$ and $T D A$. The length of event sets A$\mathrm{D}$ is larger than the one of event set $\mathrm{E}$ and therefore a full detailed description as per Table 3.1 (event set E) would have not been feasible within the text. However, all the full event sets (A-E), along with their detailed information can be found and downloaded from the 'Supplementary CSV and script files' zip file of De Luca et al. (2017). The AMAX dates for individual river basins assuming single, independent floods is denoted event set A. Event set B comprises extreme MBF episodes with severity defined in terms of number of concurrent basins involved $\left(n_{\mathrm{g}}\right)$, taking the largest episodes in the six most distinctive time windows (Figure 3.2, Table 3.1) and the 10 next largest episodes in each time window. Event set $\mathrm{C}$ contains the most extreme $L=13$-days $\mathrm{MBF}$ episode for each water year defined using $m F Y$, and set $\mathrm{D}$ is similar except defined by $T D A$. Event set $\mathrm{E}$ is the six most extreme episodes described immediately below (Figures 3.2, 3.3a-b, Table 3.1). Replicated days are removed such that days occurring in two window lengths (possible in B and E) are never counted twice. Similarly, days with $>1$ single-basin AMAX are not counted repeatedly for national-scale analyses (Figure 3.3c-d). Where different observations need to be shown basin-by-basin, multiple basins recording their AMAX are permitted to contribute on the same day (Figures 3.4-3.5).

\subsubsection{Metrics}

\subsubsection{Flood yield $(F Y)$}

Single-basin Flood Yield $(F Y)$ is calculated for each basin individually as the AMAX peak flow divided by the basin area. This can be regarded as a proxy for standardised severity of a high flow episode. The metric also allows comparison of peak flow severity between different size basins (Brown et al., 1997) and is defined as $F Y_{i}=Q_{i} / A_{i}$ where $F Y_{i}$ is Flood Yield (m3s-1km-2), $Q_{i}$ is maximum instantaneous flow (m3s-1), and $A_{i}$ is basin area $\left(\mathrm{km}_{2}\right)$, for each basin $i$. Multi-basin Flood Yield $(m F Y)$ is a plausible measure of the severity of a multi-basin episode, defined here as $\sum_{i}^{n} F Y_{i}$ where $n$ is the number of basins within an episode of specified duration (days).

\subsubsection{Basin joining time $(J t)$}

To better understand the behaviour of the basins, the joining times $(\mathrm{Jt})$ of larger $(\mathrm{A} \geq 1,000 \mathrm{~km} 2)$ and smaller $(\mathrm{A}<1,000 \mathrm{~km} 2)$ basins within the multi-basin episodes is calculated. Each basin included in a multi-basin episode is assigned a number from 1 to $d_{\max }$ (i.e. respecting chronological order) for the 
day when the basin reached the peak flow AMAX. For example, in an episode of window length $L=$ 4-days, the day count would be: 29-12-2002 = 1st day, 30-12-2002 = 2nd day, 31-12-2002 = 3rd day, 01-01-2003 $=4$ th day $\left(d_{\max }\right)$. The mean value for the smaller and larger basins is then calculated, and the value of the smaller basins is subtracted from that of the larger ones to get the difference (i.e. positive numbers indicate that larger basins join later).

\subsubsection{3 'Time to peak' modelling}

As time to peak $\left(T_{p}\right)$ is a function of hydraulic length and related to basin area, times to peak for study basins larger than $1,000 \mathrm{~km} 2$ were calculated using the REFH2.2 method (Wallingford Hydrosolutions, 2016). The model was parameterised from basin descriptors obtained from FEH-CDROM Version 3 (Wallingford: Centre for Ecology \& Hydrology, 2009).

\subsubsection{Flood Index (F-Index)}

The Flood Index (F-Index) (Wilby and Quinn, 2013) is the ratio between the frequency of floodgenerating Lamb Weather Types (LWTs) to their expected frequency in the whole period considered (i.e. 1975-2014). In other words, it shows whether a given LWT is represented disproportionately more or less on multi-basin flood days. For example, the cyclonic LWT occurs on $40.0 \%$ of days within event set E, yet only $13.4 \%$ of the time in the catalogue of all days in the same period. This yields an F-Index of $40.0 / 13.4=3.0$, indicating that this LWT occurred 3 times more than expected.

\subsubsection{Statistical testing}

\subsubsection{Multi-basin episodes}

In order to test whether the number of multi-basin episodes could have occurred by chance, a Binomial test was used under the assumption that time windows with length $L$ days, used to define episodes containing AMAX peak flows, are independent and non-overlapping. The number of trials $(n)$ is the number of basins, that are independent under the single-basin hypothesis being statistically tested (i.e. 261). The number of successes (s) is $n_{\mathrm{g}}$, the concurrent AMAX within a period of length $L$ days. The probability ( $p$ ) is the chance of each trial being successful if AMAX were randomly distributed between all possible periods of length $L$; since AMAX flows occur by definition once per gauge per year this is $L / 365$ if leap years are ignored. First, the probability of the observed coincidence in a single given period $L$ is calculated, then this is scaled up to the whole length of the record by multiplying by the number of non-overlapping window lengths in the record i.e. $p$-value scaled $=p$-value $*$ no. of days within a year * total water years / window length. 
For example, consider an $L=2$-days window with 68 concurrent AMAX observed. The parameters are: $\mathrm{s}=68, \mathrm{p}=2 / 365=0.0055$, and $\mathrm{n}=261$ giving a $\mathrm{p}$-value $<0.001$ for $\geq 68$ basins reaching their AMAX within a $L=2$-days. This is scaled up, multiplying by $365 * 40 / 2$, which still leads to a pvalue $<0.001$ making the result $99 \%$ significant.

\subsubsection{Basin joining times}

The average joining times of larger $(\mathrm{A} \geq 1,000 \mathrm{~km} 2)$ and small $(\mathrm{A}<1,000 \mathrm{~km} 2)$ basins were compared for the multi-basin episodes within event sets B, C, D and E. First, to verify the homoscedasticity (i.e. homogeneity of variances) a Fisher's F-test was applied. Second, based on the results of the F-test, to determine whether the averages were significantly different a Student's t-test (non-paired) or Welch's t-test (non-paired) was performed for each multi-basin episode, comparing larger and small basins' averages.

\subsubsection{F-Index}

The statistical significance of the F-Index based on Lamb Weather Types (LWTs) was assessed using a Binomial test. Daily observations of LWT are assumed to be independent. The number of trials (n) is the number of days within an event set. The number of successes (s) is the observed count of a LWT in the days within an event set. The probability (p) is the expected occurrence of that LWT (i.e. number of expected LWT occurrences / total LWTs occurrences in the period 1975-2014). For example, considering the $\mathrm{C}$-type within event set $\mathrm{E}, \mathrm{s}=12, \mathrm{p}=1962 / 14610=0.134292$, and $\mathrm{n}=30$, giving a p-value of $5.84 \mathrm{e}-05$ for $\geq 12$ cyclonic days occurring by chance.

\subsubsection{Multi-basin flooding and Atmospheric Rivers}

Statistical significance for co-occurrence of ARs days with event set E $d_{\max }$ was assed using a Binomial test. In this case, the number of trials (n) is the total number of temporally distinct multi-basin episodes (i.e. 5), the number of successes (s) is the observed co-occurrences (i.e. 4) and the probability (p) is the expected occurrences of days with at least one AR observation within the extended winter (6 months, October to March) and ARs threshold length of 3,000km considered in the 36-year (19792014) ARs archive (http://www.meteo.unican.es/atmospheric-rivers) (Brands et al., 2016; Dee et al., 2011) (i.e. $1936 / 6561=0.30)$. This give a p-value of 0.00224 (99\% significant) for $\geq 4 d \max$ cooccurring with ARs on the same day.

\subsubsection{SPI averages}


In order to check the statistical significance of the SPI observations within event set B, in a similar way of Section 3.3.3.2 a Fisher's F-test and t-test (non-paired) were used to compare the mean SPI on the days within the episodes to the overall record (i.e. 40-year, 1975-2014). These tests are repeated for each time window and SPI time range (i.e. 24-, 18-, 12-, 6-, 3-, and 1-month) and after Fisher's Ftest, if the variances are equal the Student's t-test was applied, otherwise Welch's t-test was used.

\subsubsection{Peak flows and very severe gales}

The interaction between extreme $m F Y$ episodes and VSG was assessed as follows. Years with both the most severe flows $(30 \%)$ and wind (50\%) were selected. Given a record of 40 years, the expected number of years where both are severe is simply $E=0.3 * 0.5 * 40=6.6$, but actually 10 were observed. Following the methodology of an earlier study (Hillier et al., 2015), a Monte Carlo simulation (with 10,000 iterations) was run to determine how often $\geq 10$ co-occurrences occur by chance. In this case, the relative p-value is set at 0.021 . In the simulation 12 (i.e. $40 * 0.3)$ random years $(1975-2014)$ were assigned to 'flood' in each 40-year realization, as were 20 (i.e. $40 * 0.5$ ) assigned to be severe for wind. The number of co-occurrences was counted for each of the 10,000 realizations to determine the probability.

The timing of episodes, within the 10 years, where both severe multi-basin flows and winds occurred together is investigated using the Binomial test. The dates, within event set $\mathrm{C}$, where the largest number of concurrent basins were detected (i.e. $d_{\max }$ ) were compared to dates where VSG occurred. Therefore, in a similar way of Section 3.3.3.1 and Section 3.3.3.3, the tasks to address were to test: i) if the occurrence of 5 out of 10 high flows on the same day of a VSG is coincidental (i.e. what is the chance of $\geq 5$ occurrences?), and ii) if the occurrence of $d_{\max }$ during a period including the day of VSG and up to 13-days afterwards is coincidental (i.e. what is the chance of $\geq 9$ occurrences?). For the first task, the number of trials (n) is the number of years (i.e. 10), the number of successes (s) correspond to the combined occurrences (i.e. 5) and the probability (p) is the chance of each trial being successful during the whole 10 years, i.e. $49 / 3650=0.0134$, where 49 are the days with a VSG and 3650 the total days within a 10-year period. Whereas for the second task (n) is still the number of years (i.e. 10), the number of successes (s) is now 9 and the probability (p) is equal to $487 / 3650=0.133$.

\subsection{Results}

\subsubsection{Characterizing severe MBF episodes}


The most extreme MBF episodes defined using $n_{\mathrm{g}}$, obtained from 19 time window lengths, comprise five temporally distinct episodes (event set E, Table 3.1). These are: $d_{\max }=27 / 12 / 1979$ (66 basins involved, $18.6 \%$ of study area, window length $L=1$-day); 30/10/2000 (68, 14.1\%, $L=2$-days); 01/01/2003 (75, 24.9\%, $L=4$-days); 02/12/1992 (96, 22\%, $L=8$-days); and 01/02/1995 (108, 46.5\%, $L=16$-days). If different time windows return the same date, the window with the largest number of concurrent AMAX is given. However, the $L=6$-days episode (30/10/2000, Figure 3.2d, Table 3.1) is included because the number of basins involved (86) and drained fraction of the study area (19.8\%) are both much larger than the $L=2$-days episode. Figure 3.2 shows the regional distribution and basinby-basin $F Y$ severity of these six episodes. 

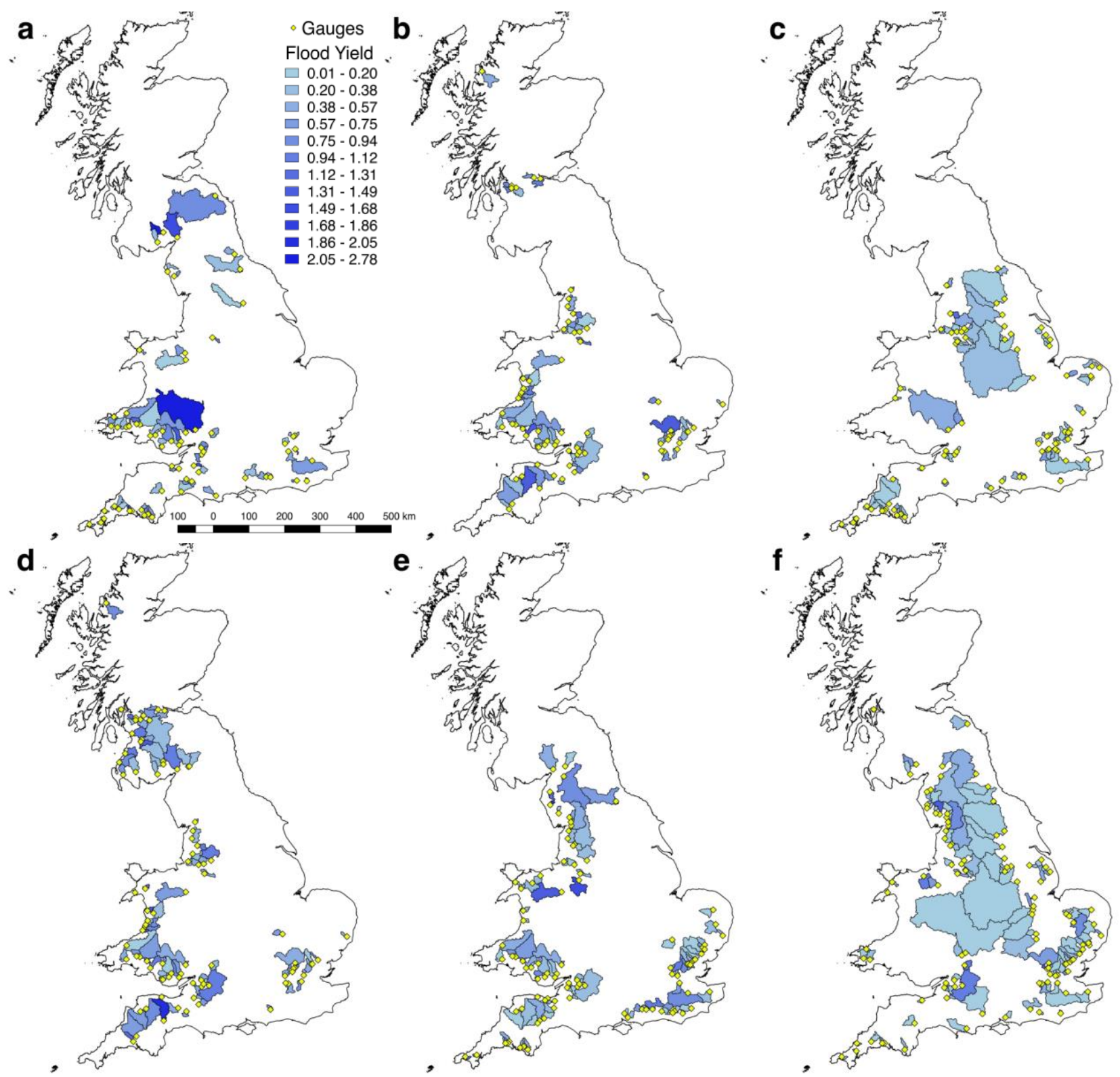

Figure 3.2 Distribution of basins contributing to the extreme MBF episodes in GB during 1975-2014 for six time window lengths $(L)$ (event set $E$ ). The maps show respectively: $(a) L=1$-day $\left(d_{\max }=27 / 12 / 1979\right)$; $(b) L=$ 2-days $\left(d_{\max }=30 / 10 / 2000\right) ;(c) L=4$-days $\left(d_{\max }=01 / 01 / 2003\right) ;(d) L=6$-days $\left(d_{\max }=30 / 10 / 2000\right) ;(e) L=8$ days $\left(d_{\max }=02 / 12 / 1992\right) ;$ and $(f) L=16$-days $\left(d_{\max }=01 / 02 / 1995\right)$. Flood Yield $(F Y)$ is a severity metric that represents each basin's peak flow AMAX normalized by the relative basin area. 
The $n_{\mathrm{g}}$ metric ranges from 66 ( $L=1$-day) to 108 ( $L=16$-days) river basins, plateauing at $L \cong 13$-days (Figure 3.3a). For all time windows, the number of co-occurrences is notably larger than expected by chance (p-value $<0.01$, Binomial test). The $T D A$ ranges from $17,787 \mathrm{~km}_{2}$ ( $L=2$-days) to $58,491 \mathrm{~km} 2(L$ $=16$-days), again plateauing at $L \cong 13$-days (Figure 3.3b). These areas correspond to $14.1 \%$ and $46.5 \%$ of the area of the 261 gauged basins respectively, or $8.5 \%$ and $27.9 \%$ of the total land area of GB (Figure 3.3b, Table 3.1). $L=13$-days defines event sets $\mathrm{C}$ and $\mathrm{D}$ as this window length captures the largest episodes whilst retaining the maximum temporal resolution. 

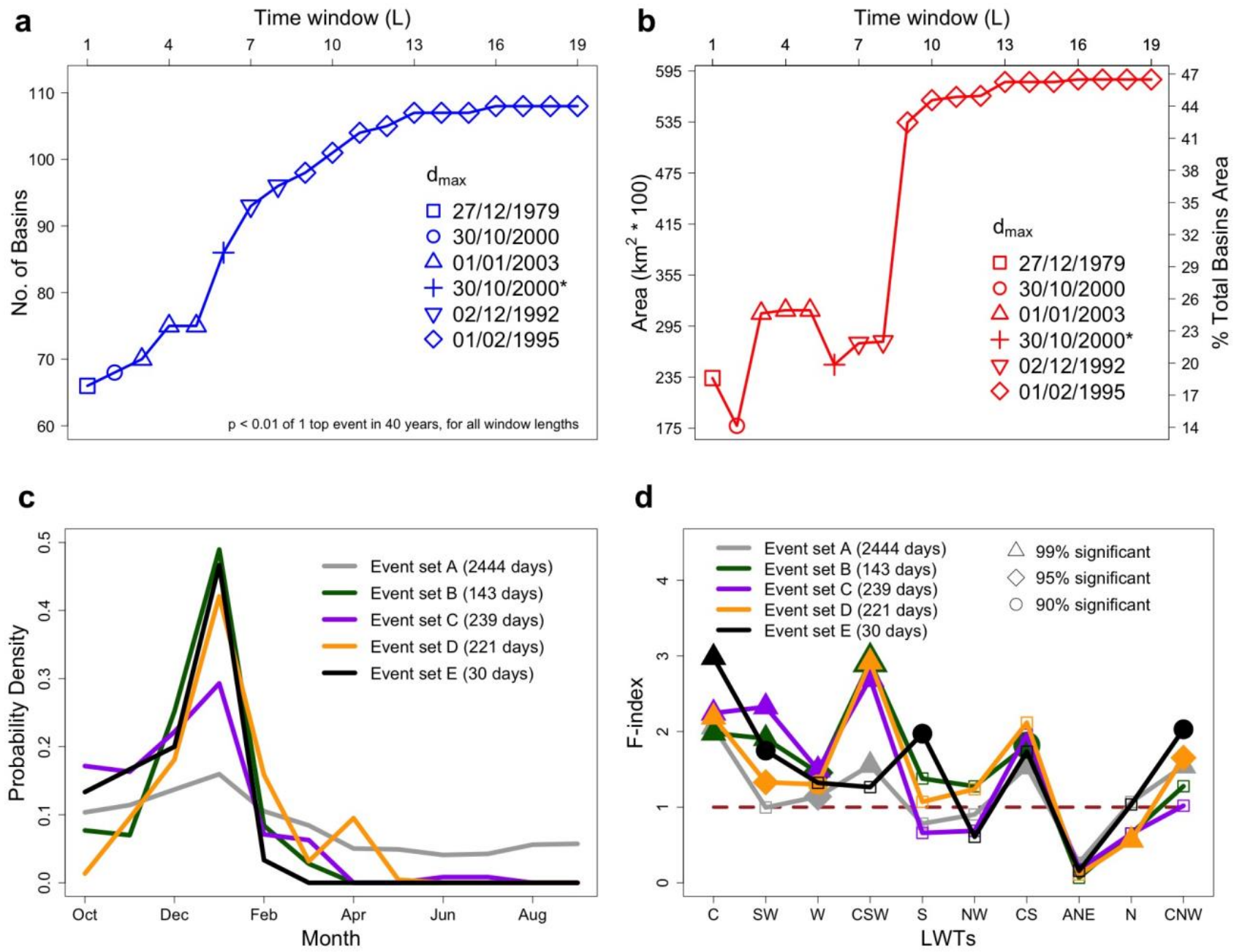

d

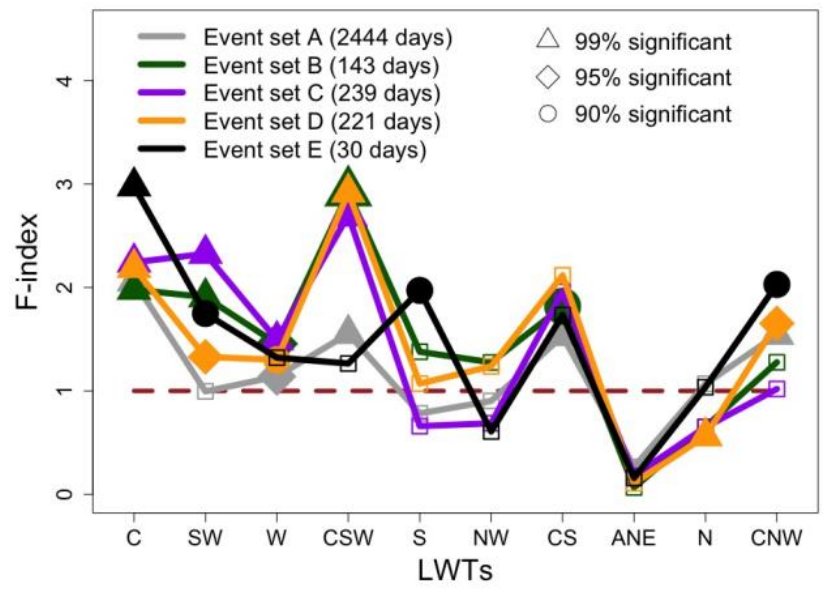

Figure 3.3 Characteristics of the extreme MBF episodes (event set E), compared to event sets $A, B, C$ and $D$. (a) Maximum number (i.e. $n_{g}$ ) of basins with concurrent AMAX versus window length $L$, defining the most extreme episodes (i.e. event set E); (b) as in (a) but for the total study area affected (i.e. TDA); (c) temporal distribution of peak flow AMAX occurrences for the extreme episodes in event sets A-E; and (d) frequency of LWTs associated with event sets A-E with respect to their expected occurrence, calculated as a Flood Index (Wilby and Quinn, 2013). Significance was determined using the Binomial test. LWTs shown are based on event set E; event sets A-D also contain other LWTs (Annex A.1.1 Figure S3.2). 
Figure 3.3c shows that the six most extensive MBF episodes (event set E) tended to occur during the winter (December to February, DJF), closely matching the pattern of event sets A-D. However, AMAX occurrences in January are more common for MBF episodes (event sets B-E) than for single-basin events (event set A). Spatially, event set E episodes impacted a substantial proportion of the study basins (Figures 3.2 and 3.4d). However, when considering more episodes (event sets B-D) the spatial distribution of basins impacted is even larger, with all the study area affected (Figure 3.4a-c). Figure 3.4 shows also that the relative frequency of AMAX occurrences is homogenously distributed across all the basins for all event sets considered. This contrasts with precipitation distributions during winters dominated by westerly or cyclonic patterns (Kendon, 2015), when rainfall tends to respectively decrease from west-to-east or is heavier in the east. 


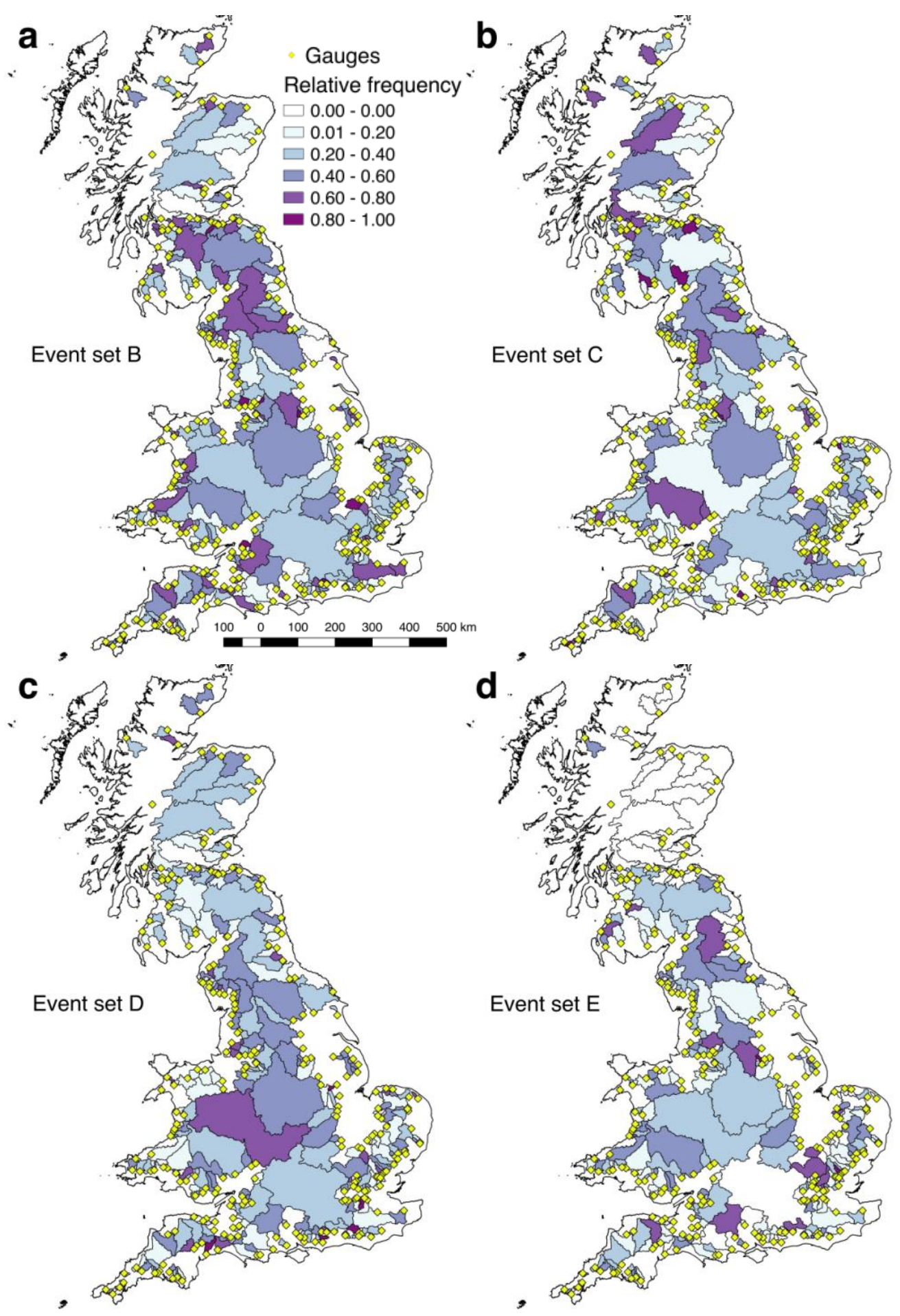

Figure 3.4 Distribution and relative frequency of occurrence of AMAX within event sets $B, C, D$ and $E$. (a) Event set $B ;(b)$ event set $C$; $(c)$ event set $D$; and $(d)$ event set $E$. The colour scale is a ratio (i.e. from 0 to 1$)$ of AMAX occurrences in a given basin relative to the basin with the largest number in that panel, with dark colours indicating most occurrences. 
The average $J t$ for larger $(A \geq 1,000 \mathrm{~km} 2)$ and smaller $(A<1,000 \mathrm{~km} 2)$ basins within MBF episodes was compared. Considering time-windows $(L)$ separately for event set E, only when $L=2$ - or 16-days do larger basins join significantly later (t-test non-paired), and the delays were modest, just 0.1 and 1.8 days respectively (Table 3.1). Event sets B-D replicate this, showing occasional significance but joining times < 48h. A $T_{p}$ response analysis (Wallingford: Centre for Ecology \& Hydrology, 2009; Wallingford Hydrosolutions, 2016) for larger basins further indicates $\mathrm{T}_{\mathrm{p}}<40 \mathrm{~h}$, again less than the $\sim 13$ day time-span that appears to define extreme MBF episodes.

For the six main episodes (event set E), maximum $F Y$ tend to be greater for short time windows. For example, it is 2.6 m3s-1 km-2 at $L=1$-day, and 2.0 m3s-1 km-2 at $L=2$-days compared with 1.9 and 1.5 m3s-1km-2 for $L=8$ - and 16-days respectively. All these basins have $T D A<71 \mathrm{~km} 2$ which is below the network mean $(482 \mathrm{~km} 2)$.

\subsubsection{Relationship to inundation episodes}

Severity measured by $n_{g}$ is a proxy for overbank flow and fluvial flood extent. Only a fraction of the basin areas will be inundated. However, the six extreme MBF episodes (event set E, Figure 3.2) all resulted in widespread flooding demonstrating the relevance of the AMAX multi-basin severity metric as a diagnostic:

- The December 1979 episode (Figure 3.2a) was the most severe in South Wales since 1960 and in some areas the worst in a century, causing extensive floods that killed four people, necessitated the evacuation of hundreds and caused millions of pounds of damage (Perry, 1980).

- The Autumn 2000 episodes (Figure 3.2b and d) were described as the most devastating in England since 1947, and associated with the wettest 12-month period since 1776 (Kelman, 2001; Marsh and Dale, 2002).

- The January 2003 episode (Figure 3.2c) was reported by the EA in FloodLink (Environment Agency, 2003) with most severe floods in the East Midlands, where the Trent basin had 118 flood warnings and 14 flood watches issued between 29/12/2002 and 03/01/2003.

- The November/December 1992 episode (Figure 3.2e) was reported by the UK Met Office (Meteorological Office, 1992) after floods impacted southern England during the night of $25_{\text {th }} / 26$ th November. However, the worst phase occurred on the 29 th, when flooding in Wales devastated homes and caused widespread road and railway disruptions. 
- The February 1995 episode (Figure 3.2f) caused severe floods on at least 7 rivers, following heavy frontal precipitation in January 1995 which was 79\% above the 1961-1990 average (Hulme, 1997; Watkins and Whyte, 2008).

\subsubsection{Relationship to atmospheric patterns}

Daily UK synoptic-scale atmospheric patterns can be characterized by LWTs (Jones et al., 2013; Lamb, 1972). The frequency of LWTs for days during extreme single- and multi-basin peak flow episodes was compared with the entire 40-year catalogue of LWTs (Figure 3.3d). In this comparison, a F-Index (Wilby and Quinn, 2013) is defined as the ratio of observed to expected frequency of LWTs. This was undertaken for event sets: A (2444 days), B (143 days), C (239 days), D (221 days) and E (30 days), excluding replicate dates. Statistical significance of the F-Index was calculated using a Binomial test.

Overall, the cyclonic (C-type) LWT is most definitively associated with the peak flows with a 99\% statistically significant F-Index $\geq 1.98$ for all event sets considered, $\sim 3$ times more than expected for event set E. The south-westerly (SW), westerly (W), and cyclonic SW (CSW) types are also associated with AMAX events (p-value <0.01, 0.05 and 0.1, Binomial test), and therefore more likely linked with widespread flooding. Southerly (S) types are significantly represented in event sets E, but not in event sets A-D (Figure 3.3d). Therefore, a pattern of C- and W-types contributing to widespread peak flows is depicted and the multi-basin event sets B-E show very similar F-Index results when compared to single-basin AMAX (event set A, Annex A.1.1 Figure S3.3).

It is also of interest if these circulation systems are particularly 'wet'. ARs are corridors of intense horizontal water vapour transport within the warm conveyor belt of ETCs (Lavers et al., 2013, 2011). $d_{\text {max }}$ dates of the event set E episodes are compared with an ARs archive (Brands et al., 2016; Dee et al., 2011). Four out of the five temporally distinct MBF episodes occurred on the same day as an AR, which on average happen on only $30 \%$ of extended (Oct-Mar) winter days (p-value $<0.01$, Binomial test). 


\begin{tabular}{|c|c|c|c|c|c|c|c|c|c|c|}
\hline $\begin{array}{c}\text { (a) } \\
\text { Time } \\
\text { window } \\
\text { length }(L, \\
\text { days) }\end{array}$ & $\begin{array}{c}\text { (b) } \\
\text { Total } \\
\text { draine } \\
\text { d area } \\
(\mathrm{TDA}, \\
\text { km2) }\end{array}$ & $\begin{array}{c}\text { (c) } \\
\text { Total } \\
\text { drained } \\
\text { area } \\
\text { (TDA, } \\
\%)\end{array}$ & $\begin{array}{c}\text { (d) } \\
\text { GB } \\
\text { area } \\
\%\end{array}$ & $\begin{array}{l}\text { (e) } \\
\text { Date }\end{array}$ & $\begin{array}{c}\text { (f) } \\
\text { No. } \\
\text { basins } \\
\text { with } \\
\text { same } \\
\text { AMAX }\end{array}$ & $\begin{array}{c}\text { (g) } \\
\text { Total } \\
\text { no. } \\
\text { basins } \\
\text { with } \\
\text { same } \\
\text { AMAX }\end{array}$ & $\begin{array}{c}\text { (h) } \\
\text { No. } \\
\text { basins } \\
\%\end{array}$ & $\begin{array}{l}\text { (i) } \\
\text { LWT }\end{array}$ & $\begin{array}{c}\text { (j) } \\
\text { Averag } \\
\text { e } \\
\text { joining } \\
\text { time (A } \\
\geq 1,000 \\
\text { km2) }\end{array}$ & $\begin{array}{c}(\mathbf{k}) \\
\text { Average } \\
\text { joining } \\
\text { time }(\mathrm{A}< \\
1,000 \\
\mathbf{k m} 2)\end{array}$ \\
\hline 1 & 23,399 & 18.6 & 11.18 & $27 / 12 / 1979$ & 66 & 66 & 25.3 & $\mathrm{C}$ & - & - \\
\hline 2 & 17,787 & 14.1 & 8.50 & $\frac{30 / 10 / 2000}{29 / 10 / 2000}$ & $\frac{62}{6}$ & 68 & 26 & $\frac{\mathrm{C}}{\mathrm{CSW}}$ & 2 & $\begin{array}{c}1.91 \\
\pm 0.0\end{array}$ \\
\hline \multirow{2}{*}{$\begin{array}{c}4 \\
\text { (3 and } 5 \\
\text { same } \\
\text { episodes } \\
\text { as 4) } \\
\end{array}$} & \multirow[t]{2}{*}{31,370} & \multirow[t]{2}{*}{24.9} & \multirow[t]{2}{*}{14.99} & $\begin{array}{l}01 / 01 / 2003 \\
31 / 12 / 2002 \\
30 / 12 / 2002 \\
\end{array}$ & $\begin{array}{c}34 \\
7 \\
29 \\
\end{array}$ & \multirow[t]{2}{*}{75} & \multirow[t]{2}{*}{28.7} & $\begin{array}{l}\mathrm{C} \\
\mathrm{S} \\
\mathrm{C} \\
\end{array}$ & \multirow[t]{2}{*}{$\begin{array}{c}2.83 \\
\pm 0.3\end{array}$} & \multirow[t]{2}{*}{$\begin{array}{r}2.94 \\
\pm 0.1\end{array}$} \\
\hline & & & & $29 / 12 / 2002$ & 5 & & & $\mathrm{C}$ & & \\
\hline \multirow{6}{*}{6} & \multirow{6}{*}{24,971} & \multirow{6}{*}{19.8} & \multirow{6}{*}{11.93} & $30 / 10 / 2000$ & 62 & \multirow{6}{*}{86} & \multirow{6}{*}{33} & $\mathrm{C}$ & \multirow{6}{*}{$\begin{array}{c}5 \\
\pm 1\end{array}$} & \multirow{6}{*}{$\begin{array}{r}4.93 \\
\pm 0.2\end{array}$} \\
\hline & & & & $29 / 12 / 2000$ & 6 & & & CSW & & \\
\hline & & & & $28 / 12 / 2000$ & 0 & & & $\mathrm{C}$ & & \\
\hline & & & & $27 / 12 / 2000$ & 2 & & & $\mathrm{~W}$ & & \\
\hline & & & & $26 / 10 / 2000$ & 0 & & & $\mathrm{~W}$ & & \\
\hline & & & & $25 / 10 / 2000$ & 16 & & & NW & & \\
\hline \multirow{8}{*}{$\begin{array}{c}8 \\
\text { (7 same } \\
\text { episode as } \\
8)\end{array}$} & \multirow{8}{*}{27,674} & & & $02 / 12 / 1992$ & 49 & & & $\mathrm{C}$ & & \\
\hline & & & & $01 / 12 / 1992$ & 1 & & & SW & & \\
\hline & & & & $30 / 11 / 1992$ & 19 & & & SW & & \\
\hline & & 220 & 1322 & $29 / 11 / 1992$ & 2 & & & $\mathrm{~S}$ & 7 & 5.91 \\
\hline & & 22.0 & 13.22 & $28 / 11 / 1992$ & 0 & 90 & 36.8 & ANE & \pm 0.8 & \pm 0.3 \\
\hline & & & & $27 / 11 / 1992$ & 5 & & & SW & & \\
\hline & & & & $26 / 11 / 1992$ & 17 & & & $\mathrm{~W}$ & & \\
\hline & & & & $25 / 11 / 1992$ & 3 & & & SW & & \\
\hline & & & & $01 / 02 / 1995$ & 19 & & & $\mathrm{~W}$ & & \\
\hline & & & & $31 / 01 / 1995$ & 16 & & & SW & & \\
\hline & & & & $30 / 01 / 1995$ & 9 & & & ANE & & \\
\hline & & & & $29 / 01 / 1995$ & 9 & & & $\mathrm{C}$ & & \\
\hline & & & & $28 / 01 / 1995$ & 10 & & & $\mathrm{C}$ & & \\
\hline 16 & & & & $27 / 01 / 1995$ & 16 & & & $\mathrm{~S}$ & & \\
\hline (9 to 15 & & & & $26 / 01 / 1995$ & 14 & & & $\mathrm{~N}$ & & \\
\hline & & & & $25 / 01 / 1995$ & 3 & & & $\mathrm{C}$ & 13.73 & 11.97 \\
\hline $\begin{array}{l}19 \text { same } \\
\text { enisodes }\end{array}$ & 58,491 & 46.9 & 27.94 & $24 / 01 / 1995$ & 2 & 108 & 41.4 & $\mathrm{~W}$ & \pm 0.9 & \pm 0.3 \\
\hline $\begin{array}{l}\text { episodes } \\
\text { as 16) }\end{array}$ & & & & $23 / 01 / 1995$ & 3 & & & $\mathrm{CNW}$ & & \\
\hline & & & & $22 / 01 / 1995$ & 3 & & & $\mathrm{C}$ & & \\
\hline & & & & $21 / 01 / 1995$ & 1 & & & $\mathrm{C}$ & & \\
\hline & & & & $20 / 01 / 1995$ & 2 & & & $\mathrm{C}$ & & \\
\hline & & & & 19/01/1995 & 0 & & & $\mathrm{CS}$ & & \\
\hline & & & & $18 / 01 / 1995$ & 0 & & & SW & & \\
\hline & & & & $17 / 01 / 1995$ & 1 & & & $\mathrm{CS}$ & & \\
\hline
\end{tabular}


Table 3.1 Extreme MBF episodes in GB during 1975-2014 (event set E). Observations are derived from 19 time windows up to 18 days prior $d_{\max }$; see main text for details. (a) Window length $(L)$ in days; (b) Total drained area (TDA, km2) involved in each episode (i.e. sum of the area of all involved basins); (c) Percentage of TDA of the 261 basins affected by each episode; (d) Percentage of GB land area affected by each episode; ( $e$ ) Dates of episodes, where the top row represents $d_{\max }$; $(f)$ Number of basins with peak flow AMAX registered on the same day; $(g)$ Total number of basins with peak flow AMAX per episode; ( $h$ ) Percentage of total number of basins (out of 261) with concurrent AMAX per episode; (i) Daily LWT; (j) Average joining time, within an episode, for larger basins ( $A \geq 1,000 \mathrm{~km} 2)$; ( $k$ ) Average joining time for small basins $(A<1,000 \mathrm{~km} 2)$. In $(j)$ and (k) uncertainties are 1 standard error of the mean.

\subsubsection{Relationship with antecedent soil moisture conditions}

High soil moisture content increases the likelihood of flooding. The SPI (McKee T.B., Doesken N.J., 1995; McKee et al., 1993) is widely used as a proxy for this physical property and 3-24 month SPI values are distinctively high for historical episodes (Du et al., 2013; Seiler et al., 2002; Wang et al., 2015). Whilst the sample of episodes in event set $\mathrm{E}$ is too small to show a pattern, SPI aggregated across impacted basins (Tanguy M., Kral F., Fry M., Svensson C., 2015) is significantly (p-value $<0.05$, t-test non-paired) higher than average across all $L$ for event set B (p-value $<<0.01$ ), increasing with $L$ (Figure 3.5). Event set C, by incorporating a forced regularized annual sampling, demonstrates that flood magnitude is greater in 'wet' spells (SPI >0.5) than 'dry' periods (SPI <-0.5) with mean $m F Y=26.9 \pm 3.4(1 \sigma)$ and 17.1 $\pm 1.3(1 \sigma)$ respectively for SPI-12. Indeed, for this comparison, all except SPI-1 are significant (p-value <0.05, t-test, 2-tailed). Event set D (based on TDA) shows no signal for this well-established flood-SPI connection, suggesting that the metric based on $m F Y$ (event set C) might better reflect physical processes. 


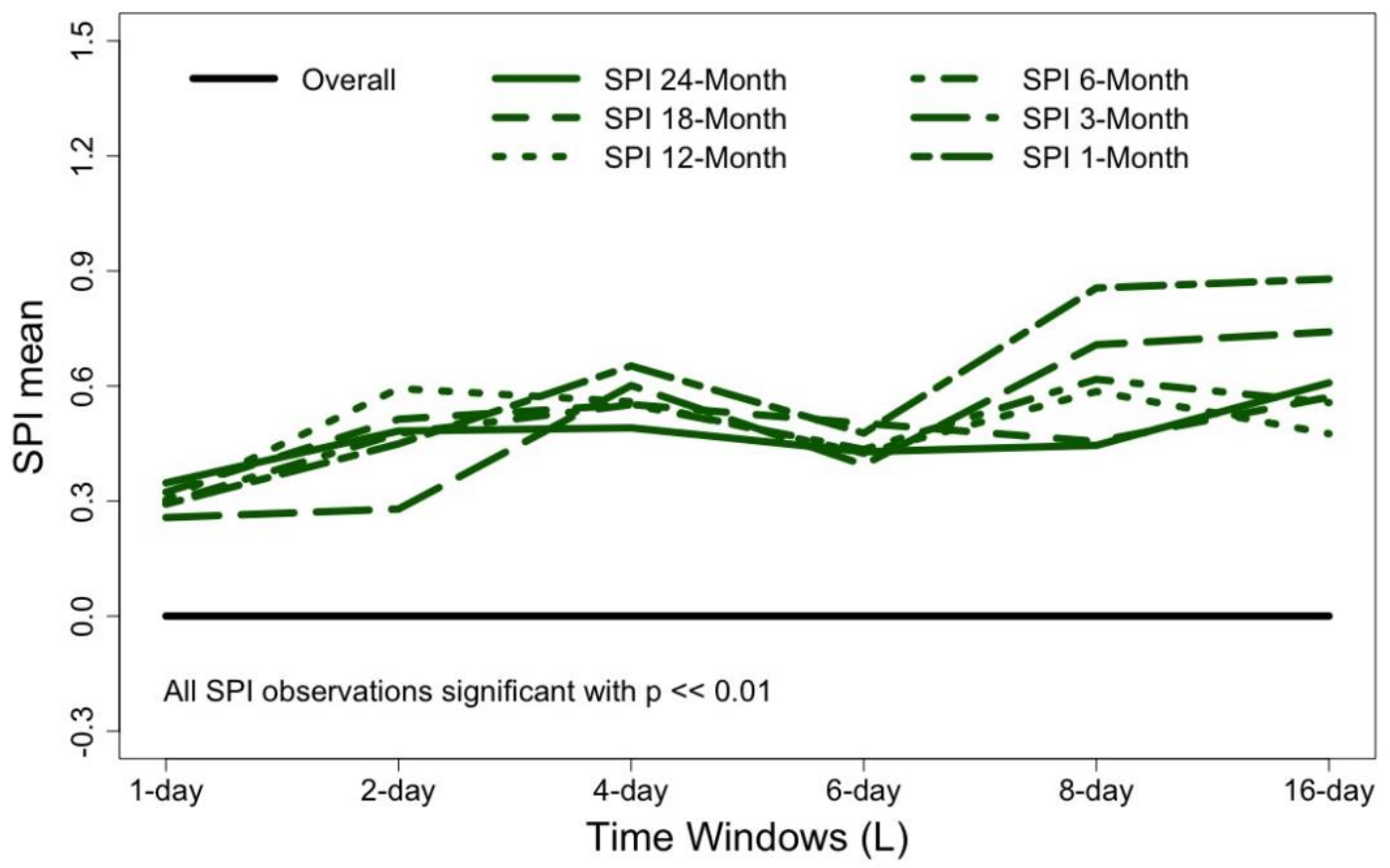

Figure 3.5 Mean Standardized Precipitation Index (SPI) for episodes within event set B for each window length (L) and SPI time scale (24-1 Months). Green lines are episode SPI averages and the black line represents the overall (40-year, 1975-2014) SPI average i.e. zero by definition. All episodes have SPI that are significantly different from the long-term mean at $99 \%$ level ( $t$-test, not paired).

\subsubsection{Relationship to very severe gales}

Flooding and severe wind have been reported for some ETCs impacting western Europe (Barker et al., 2016; McCarthy et al., 2016). A potential association between extreme MBF episodes and severe storms was, therefore, investigated. In a year-by-year analysis the most extreme $L=13$-days $m F Y$ episodes (event set C) correlates positively with the number of days with VSG as defined by the Jenkinson Gale Index (Jenkinson and Collison, 1977; Jones et al., 2013) in that year $(r=0.41$, p-value $=0.0088,2$-tailed t-test) (Figure 3.6). Taking the most severe $50 \%$ and $30 \%$ of years for wind and flow respectively, co-occurrence is expected 6.6 times in 40 years, but 10 are observed (p-value $=0.021$; Monte Carlo simulation with $n=10,000$ ), making coincidence of the extremes $52 \%$ more likely than would be expected by chance.

Furthermore, the timing of these episodes provides insight to the physical processes at work. For 5 out of 10 observed co-occurrences, the most extreme peak flows recorded on $d_{\max }$ are on a day with VSG, and 9 of 10 peak flows are within 0-13 days after a VSG day (p-value $\ll 0.001$; Binomial test). This contrasts with 0 out of 10 peak flow episodes found in the preceding 0-13 days of a VSG day. In 
agreement with the flood-SPI analysis, the relationship is notably less strong for event set D (based on $T D A$ ), indicating that $m F Y$ may better reflects of physical processes in storm systems.

Wet ground is a pre-requisite to the most severe peak flow episodes, but there is also a link with gales. Six out of the 10 most severe episodes have a 12-Month SPI between +0.4 and +1.1 (Figure 3.6, white circles), whereas less severe episodes tend to show a negative SPI-12 (Figure 3.6, black circles). The two outliers in Figure 3.6 (1983 and 2014) reflect previous studies (Befort et al., 2016; Kendon, 2015; Matthews et al., 2014; Muchan et al., 2015; Wild et al., 2015) that showed that the number of cyclones were particularly high over the GB during these years. However, the largest $m F Y$ for these two episodes may be depressed by the AMAX measure of extremeness which, by definition, limits the number of occurrences per year. Therefore, these observations are likely valid given the analytical method used.
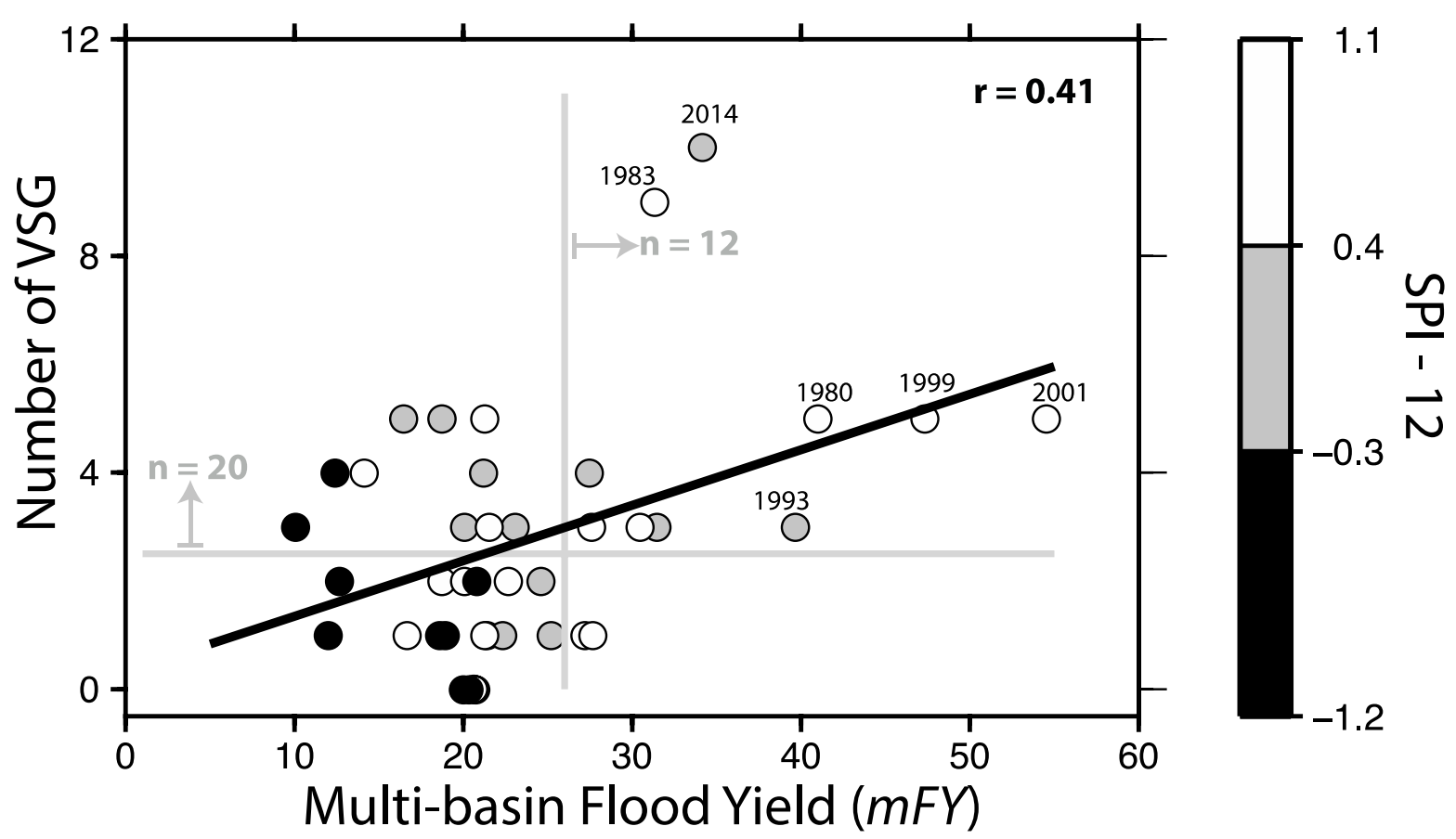

Figure 3.6 Number of Very Severe Gales (VSGs) versus extreme multi-basin Flood Yield (mFY) episodes belonging to event set $C$ for each water year (1975-2014). Black circles =12-Month SPI <-0.3; Grey circles $=-0.3<12$-Month SPI $<0.4 ;$ White circles $=12$-Month SPI >0.4. $n=20$ represents the $50 \%$ most extreme $V S G$ and $n=12$ the $30 \%$ most extreme $m F Y$ episodes. 


\subsection{Discussion}

\subsubsection{A new multi-basin approach}

Various diagnostics for the evaluation of MBF episodes were presented here. The first metric detects key 'episodes' by summing the concurrent number of basins (i.e. $n g$ ) attaining their AMAX within a given time window, then ranking the episodes based on $n_{g}$. Once basins contributing to each episode are identified other metrics can characterize different aspects of the episodes. When episodes are identified in terms of $n_{g}$, this gives perhaps undue weight to small basins, but TDA emphasizes larger rivers. The $m F Y$ can either weight small basins, when calculated as here or large ones if area and flow were each summed before dividing them. All are practical options, but awareness of any biases and use of multiple metrics is recommended to ensure robust insights.

There are various advantages with this approach to MBF analysis. First, it enables the identification of extreme peak-flow episodes that are driven by persistent rain-bearing weather systems by accounting for variations in time-lags before peak flow that depend on precipitation properties, basin area and geology. Second, it provides a national-scale flood measure allowing more meaningful comparison with synoptic-scale weather patterns than at the scale of individual basins, no matter how large (Hillier et al., 2015). Third, whichever metric is selected a return period that is applicable across a whole country can be estimated.

A single, national rather than basin-scale, return period has a potentially important role in risk communication. Such metrics could address the question often posed to flood managers: "Why is there a 1 in 100-year flood event every year?" This impression arises because return period estimates are traditionally based on flows at a single gauge. The MBF metrics proposed in this chapter would yield the 1-in-100 year episode based on a return period estimate that integrates information across all basins in a network.

\subsubsection{Widespread concurrent impacts}

Answers to the first and second research questions posed in Section 3.1 are here presented and discussed. Results show that extreme MBF episodes can affect large areas (Figure 3.2), with likely commensurate damages (Environment Agency, 2003; Hulme, 1997; Kelman, 2001; Marsh and Dale, 2002; Meteorological Office, 1992; Perry, 1980; Watkins and Whyte, 2008). For instance, the $L=16$ days episode captures $\sim 46 \%$ of the study area (or $\sim 27 \%$ of GB) with 108 basins concurrently reaching their AMAX (Figures 3.2f, 3.3a-b, Table 3.1). Aspects of the physical processes driving these 
widespread episodes appear similar to those deduced from single-basin studies (Barker et al., 2016; Blöschl et al., 2015; Huntingford et al., 2014; Kendon, 2015; Merz, R. Blöschl, 2003; Merz et al., 2014; Muchan et al., 2015; Nied et al., 2016; Schaller et al., 2016). First, W- and C-type LWTs associated with MBF (Figure 3.3d, Table 3.1) have been linked to frequent floods (Pattison and Lane, 2012; Wilby and Quinn, 2013), the wettest winters in England and Wales (Kendon, 2015; Kendon and McCarthy, 2015), and $>80 \%$ of extreme flows on the River Eden (UK) (Pattison and Lane, 2012). The W-type, in particular, represents one of the main drivers of high rainfall and flows in the UK (Hannaford and Marsh, 2008; Pattison and Lane, 2012) as well as flooding throughout central Europe (Pattison and Lane, 2012). Second, maximum $F Y$ occur in smaller basins $(<71 \mathrm{~km} 2)$. This is consistent with higher expected flood severity in smaller basins which respond more rapidly to any given storm. Third, MBF is larger (by $m F Y$ ) in wet years (SPI $>0.5$ ), and in longer time windows when SPI is higher.

The observation that single-basin flooding in GB occurs mostly during winter also applies to MBF episodes (Figure 3.3c). This is due to frequent storms and their associated precipitation (Matthews et al., 2018, 2016b), combined with lower evapotranspiration, and wetter antecedent soil conditions (Figure 3.5) that ultimately combine to generate higher flows (Blöschl et al., 2015; Huntingford et al., 2014). However, the largest MBF episodes are most common in January (Figure 3.3c), when the most favourable conditions (C, SW, W and CSW circulation types) are more likely.

A key feature that distinguishes large MBF episodes from their single-basin counterparts is their duration (i.e. $\cong 13$-days, Figure $3.3 \mathrm{a}-\mathrm{b}$ ). This is greater than currently accounted for in other studies (Cameron et al., 1999), and indicates that a notable 'memory' in the physical system is required. With a $T_{\mathrm{p}}<40 \mathrm{~h}$ (Wallingford: Centre for Ecology \& Hydrology, 2009; Wallingford Hydrosolutions, 2016), this cannot be within the channelized flow paths, a view supported empirically by larger basins joining episodes at essentially the same time as smaller ones (Table 3.1 and Annex A.1.1 Figure S3.4). This observation also rules out reservoirs delaying flow outside of the channels, and is reconciled by the fact that concentration time increases with basin area (Grimaldi et al., 2016). Thus, the memory should exist in either antecedent soil or groundwater levels (Du et al., 2013; Seiler et al., 2002; Wang et al., 2015) (Figures 3.5-3.6), or in persistent atmospheric patterns during notably wet years (Barker et al., 2016; Huntingford et al., 2014; Kendon, 2015; Matthews et al., 2018; McCarthy et al., 2016; Muchan et al., 2015; Wild et al., 2015). These elements of memory likely exist for larger European rivers (e.g. 
Rhine), although they are less easily decoupled because time-scales attributable to the processes overlap more.

\subsubsection{Compounding flood and wind impacts}

The third research question highlighted in Section 3.1 is also answered and discussed in this subparagraph. ETCs were identified as a driver of MBF episodes (via cyclonic LWT and ARs). When considering these high flows in terms of extreme $m F Y$ for each water year within $L=13$-days (event set C), a relationship with damaging winds predominantly caused by ETCs is also demonstrated. A significantly positive correlation exists between VSG and MBF episodes, with co-occurrence of extremes 52\% more likely than by chance and high flows occurring within 0-13 days after a VSG day. Building on case studies of notable years (Huntingford et al., 2014; Kendon, 2015; Matthews et al., 2014; Muchan et al., 2015; Schaller et al., 2016; Wild et al., 2015) and the Trent basin in central England (Hillier et al., 2015), this is the first systematic, national-scale evidence that the severest aspects of wet and windy winters tend to co-occur. Often these phenomena are viewed separately: severe ETCs bring extreme winds (Pinto et al., 2009) whilst slower moving, less windy ETCs bring large accumulated rainfall totals and extensive flooding in GB (Burt et al., 2015; Matthews et al., 2016b; Wilby and Quinn, 2013). Thus, the evidence of coincident widespread flood and wind on the same day in 5 out of 10 years and within 13 days in another 4 of those years contradicts a prevailing view that Storm Desmond was exceptional in bringing both very severe wind and widespread flooding (Barker et al., 2016; Matthews et al., 2018; McCarthy et al., 2016). These findings also highlight the importance of considering longer time-lags when assessing dependencies between weather-driven hazards where both may not occur in the same defined extreme episode. As far the author is aware, this is the first statistical evidence of a time-lagged link between widespread flooding and severe wind for any nation. This methodology also enables potential detection of such inter-dependencies elsewhere. Moreover, as for single-basin floods (Lavers et al., 2011), extreme MBF episodes cooccurred with ARs.

One implication of coincident floods and severe winds is that worst-case years are likely more severe than previously thought. With the association apparently strongest for the most extreme episodes, the effect of this co-occurrence on annualized aggregate insurance losses could exceed the $£ 0.3$ billion reported for UK domestic properties for a 16-year return period event (Hillier et al., 2015). Moreover, GB is located beneath the North Atlantic storm track and is, therefore, affected by the passage of ETCs (Matthews et al., 2016b) which bring extreme winds (Matthews et al., 2018; Pinto et al., 2009) that can subsequently affect central Europe (Donat M. G., Leckebusch G. C., Pinto J. G., 2010). Since 
ETCs can continue to strengthen after landfall, this effect may extend to a much larger physical and financial scale than the GB alone. Furthermore, there is a likely three-way association between widespread flooding, severe wind and storm surges that warrants investigation.

\subsubsection{Operational implications}

The EA is responsible for contingency planning, forecasting and managing the consequences of widespread flood episodes. Regional 'footprints' of past severe episodes (Figure 3.2) reveal the extent to which authorities in neighbouring areas could be impacted simultaneously. This is relevant when coordinating and sharing equipment and personnel during such episodes. For instance, the Midlands region of the EA lies in a pivotal location since it may be called upon to provide resources to affected areas to the North and South. During the severe flooding in December 2015, personnel and equipment were drawn from regions hundreds of kilometres away from the epicentre of Northwest England and Southern Scotland. This might not be feasible in the event of a MBF episode on the scale of January/February 1995 (Figure 3.2f). However, knowledge of the likelihood and pattern of MBF provides a basis for role-play exercises as part of the contingency planning for such episodes.

Both the UK National Flood Resilience Review (HM Government, 2016) and CCRA (ASC, 2016) recognise interdependencies between critical networks (e.g. electricity, water and transport) and the need to manage indirect flood impacts on the economy. However, their emphasis remains on integrated, yet single-basin solutions involving 'natural' flood management, improved property- and asset-level resilience, and planning controls. Widespread flooding in Australia in 2011, and multiple events in Central Europe since 2002, show the need for a higher-level strategy for managing extensive, transboundary flooding (Wilby and Keenan, 2012). Moreover, the likelihood of MBF episodes could increase with ETCs intensity and ARs frequency and magnitude expected to rise under anthropogenic climate change (Donat M. G., Leckebusch G. C., Pinto J. G., 2010; Donat et al., 2010; Lavers et al., 2013; Matthews et al., 2018, 2016b; Ulbrich et al., 2009; Zappa et al., 2013).

\subsubsection{Storms since 2014}

The analysis conducted in this chapter ends in water-year 2014 and since then, the UK was affected by the passage of other impactful ETCs, causing socio-economic disruption. During storm season 2015-2016, when the Met Office and Met Éireann officially started to provide a name to storms, there were 11 ETCs impacting the UK and/or Ireland (Met Office). The most noticeable was Storm Desmond (Burt et al., 2016; Matthews et al., 2018; Wilby and Barker, 2016), that caused recordbreaking flooding over Cumbria and Lancashire, with 5,200 homes flooded and more than 50,000 
without power in north-eastern England (Met Office). During storm season 2016-2017 only five ETCs were named (Met Office). The most impactful was Storm Doris, which was characterised by strong wind gusts up to $94 \mathrm{mph}$ in north Wales and heavy snowfall in Scotland. Lastly, in 2017-2018 season a total of 8 storms impacted the UK and/or Ireland (Met Office). In this 10-month period the most significant storms were Eleanor and Hector. The former impacted the UK during January 2018 with wind gusts up to $100 \mathrm{mph}$ in Cumbria, causing widespread transport disruption and power cuts, along with storm surges. On the other hand, the latter occurred in June 2018 and was characterised by strong winds and heavy rainfall in the UK and Ireland, eventually leading to road and rail disruption and fallen trees (Met Office).

\subsection{Summary}

The focus of the above research is on the smallest geographical domain of the thesis, which is Great Britain (GB), yet contributes to knowledge about the interactions between extreme multi-basin floods and extra-tropical cyclones (ETCs) (De Luca et al., 2017). The data used belong to observations recorded within the 1975-2014 period and physical variables considered are peak river flows as a proxy for flooding, standardized precipitation index (SPI) for representing soil moisture conditions (McKee T.B., Doesken N.J., 1995; McKee et al., 1993) and weather patterns (LWTs) (Jenkinson and Collison, 1977; Jones et al., 1993; Lamb, 1972), very severe gales (VSGs) (Jenkinson and Collison, 1977; Jones et al., 2013) and atmospheric rivers (ARs) (Brands et al., 2016; Dee et al., 2011) as a measure of storminess.

The key findings can be summarised as follows: i) the greatest number of river basins concurrently reaching their peak river flow annual maximum (AMAX) is up to 108 within a time-window of 16 days; ii) the total area impacted by such multi-basin flooding event is $\sim 46 \%$ of the studied area; iii) the extreme multi-basin events detected by the procedures match those reported flooding episodes that have caused significant socio-economic impacts; iv) most extreme multi-basin flooding is found to be driven by cyclonic and westerly LWTs, ARs and precipitation falling onto previously saturated ground; and v) peak river flows (AMAX) tend to occur within 0-13 days of VSGs. These results may have significant implications for policy makers, stakeholders, insurance industry and emergency managers, as they provide a clear evidence of an observed multi-hazards scenario, namely large-scale flooding concurrently impacting the country with severe wind events within a time-window of about two weeks. Moreover, the simple methods presented in this chapter can be also applied to other regions 
that may not be so well resourced as GB. This could lead to improved preparedness, emergency planning, capital insured and more broadly disaster risk reduction (DRR) measures.

The following Chapter examines observed and future seasonal projections of persistence and frequency of the same LWTs (Jenkinson and Collison, 1977; Jones et al., 1993; Lamb, 1972) and how these may be proxies for multi-hazards (De Luca et al., 2019a). The study focusses on a larger geographical domain, now the British Isles (BI), and much longer time-span covering 1950 to 2100. This provides a forward look to multi-hazards, such as flooding and storms, heatwaves/air pollution and droughts. 


\section{Chapter 4}

\section{Past and projected weather pattern persistence associated with multi-hazards in the British Isles}

\subsection{Introduction}

Persistent weather patterns can translate into hazards such as heatwaves, poor air quality, drought, wildfires and episodes of flooding (Coumou et al., 2018; Francis and Skific, 2015; Francis and Vavrus, 2015, 2012), with significant socio-economic losses (Munich Re, 2019, 2015). Examples of such impactful episodes include the 2003 and 2010 European summer heatwaves that led to more than 100,000 deaths, reduced gross primary productivity of crops and, in the latter episode over Russia, about US\$ 15 billion economic losses (Barriopedro et al., 2011; Bastos et al., 2014; Le Tertre et al., 2006; Stott et al., 2004). Similarly, summer 2013 in eastern China, was the hottest ever recorded in that region, with persistent and widespread heatwaves and droughts causing severe socio-economic impacts amounting to 59 billion RMB in losses (Sun et al., 2014). Conversely, the extremely wet and stormy 2013/14 winter over the United Kingdom (UK) was characterised by the passage of numerous low-pressure systems causing extensive pluvial, fluvial, coastal and groundwater flooding along with severe gales (Kendon and McCarthy, 2015; Matthews et al., 2014; Muchan et al., 2015).

A growing body of literature is discussing possible dynamical mechanisms linking Arctic Amplification (AA) (Screen and Simmonds, 2010), i.e. the faster warming of the Arctic compared to the global scale, with more persistent weather patterns across the northern hemisphere mid-latitudes (Cohen et al., 2018, 2014; Coumou et al., 2018; Francis and Skific, 2015; Francis and Vavrus, 2015, 2012). The influence of AA on the polar jet stream is the dynamical mechanism that has attracted most attention from the media, policy makers and scientists. In fact, since AA began to be observed in the late 1980s, the jet stream has assumed a wavier and weaker character, which accounts for more persistent weather patterns, and hence impactful extreme events in the northern mid-latitudes (Cohen et al., 2018, 2014; Di Capua and Coumou, 2016; Francis, 2017). Meanwhile, analysis of weather type occurrence and persistence in historical and $21_{\text {st }}$ century climate model runs, under different Representative Concentration Pathways (RCPs), is becoming relevant for assessing the dynamical realism of models as well for describing associated weather and climate extremes (Franzke, 2013; Hannachi et al., 2017; Murawski et al., 2016; Sillmann et al., 2017). By focusing on weather type persistence both model realism and associated phenomena can be examined. 
Previous studies have investigated linkages between weather patterns (or large-scale atmospheric circulation) and local extreme events, such as heavy rainfall, storms, floods and heatwaves (Conticello et al., 2018; De Luca et al., 2017; Farnham et al., 2018; Matthews et al., 2016b; Merz et al., 2014; Murawski et al., 2018, 2016; Pattison and Lane, 2012; Wilby et al., 2011). The conventional approach to flood analysis at the single catchment scale is being extended to frameworks with inter-related hazards, driven by global climate modes, covering multiple catchments (Merz et al., 2014). Others show that the bias in simulating regional extreme precipitation days by an Atmosphere-Ocean General Circulation Model (AOGCM) is reduced by applying atmospheric circulation indices (Farnham et al., 2018). Moreover, weather patterns extracted from AOGCMs have also been used to downscale local climate variables, such as temperature, precipitation, radiation and humidity at local scales (Murawski et al., 2016; Wilby and Wigley, 1997; Xu et al., 2007). However, AOGCMs vary in their ability to simulate the frequency, seasonality and persistence of weather patterns at regional scales (Murawski et al., 2016, 2018). Some studies have linked heavy precipitation events to atmospheric circulation states, such as the $850 \mathrm{hPa}$ geopotential height field or integrated vapour transport (IVT) (Conticello et al., 2018), and found connections between selected weather patterns and multi-basin flooding driven by ETCs (De Luca et al., 2017). Others have used weather patterns to quantify changes in the strength of the nocturnal Urban Heat Island (UHI) - a phenomenon that may be associated with combined heatwave and air pollution events within cities (Wilby et al., 2011).

The research questions addressed within this chapter are the followings:

1) How has persistence in weather pattens changed historically in the BI?

2) To what extent can Atmosphere-Ocean General Circulation Models (AOGCMs) reproduce observed weather pattern persistence over the BI?

3) How are weather pattern persistence and frequency expected to change in the future under different Representative Concentration Pathways (RCPs)?

4) How changes in future weather type persistence might translate into changed risk of winter flood-wind and summer heatwave-air pollution concurrent hazards?

Previous evaluations for Europe and the British Isles (BI) show that Coupled Model Intercomparison Project Phase 5 (CMIP5) AOGCMs generally reproduce synoptic-scale weather patterns, calculated using daily sea-level pressure (SLP) fields, but there are recognized biases (Otero et al., 2018; Stryhal 
and Huth, 2018). For example, CMIP5 AOGCMs are not yet able to simulate correctly the number of anticyclonic (A-type) patterns and hence blocking episodes, with the former being underestimated in northern Europe and the BI, but overestimated in southern Europe (Otero et al., 2018; Stryhal and Huth, 2018; Woollings et al., 2018). Other biases are found for cyclonic (C-type) and westerly (Wtype) occurrences, with both being overestimated across Europe (Otero et al., 2018). These studies also examined future changes in frequency of weather patterns and blocking episodes by comparing historical conditions with RCP8.5, to determine how such changes might affect European temperatures. The A-type is projected to increase significantly over the BI during all seasons except winter (DJF), the C-type to decrease in all seasons, and the W-type to increase except in summer (JJA) by the end of the century (Otero et al., 2018). Overall, blocking episodes are projected to decrease for the BI in DJF and JJA by 2061-2090 (RCP8.5) (Woollings et al., 2018).

In this chapter, these analyses were extended by assessing the ability of a CMIP5 (Taylor et al., 2011) multi-model sub-ensemble (MME) of 10 AOGCMs at reproducing historical seasonal persistence of daily weather patterns, here identified as Lamb Weather Types (LWTs) over the BI (Jenkinson and Collison, 1977; Jones et al., 2013, 1993; Lamb, 1972). Two-day persistence is defined as the probability that a given LWT will occur on any two successive days and therefore such definition lays down the basis for answering research questions 1-4. Climate model simulations of historic weather patterns are compared with those derived from Twentieth Century Reanalysis (20CR) (Compo et al., 2011), National Centers for Environmental Predictions/National Center for Atmospheric Research (NCEP/NCAR) (Kalnay et al., 1996) reanalyses, and Lamb's catalogue of subjectively defined weather types (Hulme and Barrow, 1997; Lamb, 1972). This investigation made possible to answer research questions 1-2. Furthermore, for addressing research question 3, an investigation about how persistence and seasonal frequencies are projected to change within the full 21 st century under RCP8.5 and RCP4.5, with persistence assessed for both the MME mean (MMEM) and individual AOGCMs is presented. Lastly, the implications of future multi-hazards, here identified as nearly concurrent multibasin flooding and ETCs impacting Great Britain (GB) in winter (De Luca et al., 2017) or combined summer heatwave and poor air quality events over London (Wilby et al., 2011) are quantified and discussed. Thus, for fulfilling research question 4, two multi-hazard metrics are applied, along with their evaluation under RCP8.5 and RCP4.5 projections up to 2100: likelihood of (1) multi-basin flooding (F-Score) and (2) changing intensity of the nocturnal UHI. The F-Score metric strictly relates to the F-Index (Wilby and Quinn, 2013) used and computed for concurrent flood-wind impacts over GB (see Chapter 3 and De Luca et al., 2017). 


\subsection{Methods and Data}

\subsubsection{Lamb Weather Types (LWTs)}

Daily atmospheric sea-level pressure (SLP) patterns are categorized using the system of LWTs (Lamb, 1972) via an objective classification scheme centred over the BI (Figure 4.1) (Jenkinson and Collison, 1977; Jones et al., 1993). Choice of the LWTs objective scheme is justified by the fact that this methodology and weather typing classification was originally developed for the BI. LWTs of similar airflow properties are derived from a $5^{\circ}$ by $10^{\circ}$ latitude-longitude grid array (Figure 4.1) and computed from daily (12 UTC) SLP values at each grid point. The airflow characteristics are expressed by the following set of equations, where the integers in bold correspond to the grid point reference numbers in Figure 4.1:

$$
\begin{array}{ll}
W=\frac{1}{2}(\mathbf{1 2}+\mathbf{1 3})-\frac{1}{2}(\mathbf{4}+\mathbf{5}) & \text { (westerly flow) } \\
S=1.74\left[\frac{1}{4}(\mathbf{5}+2.0 \times \mathbf{9}+\mathbf{1 3})-\frac{1}{4}(\mathbf{4}+2.0 \times \mathbf{8}+\mathbf{1 2})\right] & \text { (southerly flow) } \\
F=\left(S^{2}+W^{2}\right)^{1 / 2} & \text { (resultant flow) }
\end{array}
$$

$Z S=1.52\left[\begin{array}{c}\frac{1}{4}(\mathbf{6}+2.0 \times \mathbf{1 0}+\mathbf{1 4})-\frac{1}{4}(\mathbf{5}+2.0 \times \mathbf{9}+\mathbf{1 3})-\frac{1}{4}(\mathbf{4}+2.0 \times \mathbf{8}+\mathbf{1 2}) \\ +\frac{1}{4}(\mathbf{3}+2.0 \times \mathbf{7}+\mathbf{1 1})\end{array}\right]$

(southerly shear vorticity)

$Z=Z W+Z S$

(total shear vorticity)

Flow units are derived from the geostrophic approximation (each equivalent to 1.2 knots) and they are, along with the geostrophic vorticity units, expressed as hPa per 10 o latitude at $55 \mathrm{oN}$ (100 units are equivalent to $0.55 \times 10-4=0.46$ times the Coriolis parameter at $55 \mathrm{oN}$ ). Three coefficients are used within equations $(4.2,4.4$ and 4.5$)$ to account for variations in relative grid spacing at different latitudes with 
latitude $(\psi)$ here set as 55o (Jenkinson and Collison, 1977): $S$ is multiplied by 1.74 , derived from $1 / \cos$ $(\psi) ; Z W, 1.07$ and 0.95 from $\sin (\psi) / \sin \left(\psi-5^{\circ}\right)$ and $\sin (\psi) / \sin \left(\psi+5^{\circ}\right) ; Z S, 1.52$ from $1 / 2(\cos (\psi) 2)$.

The last step for defining LWTs is to apply five rules (Jenkinson and Collison, 1977; Jones et al., 1993; Lamb, 1972):

i) the flow direction is given by $\tan -1(\mathrm{~W} / \mathrm{S})$ and is calculated on an eight-point compass with 45o per sector. If $\mathrm{W}$ is positive, add 180 . Thus, the $\mathrm{W}$-type occurs between $247.5^{\circ}$ and $292.5^{\circ}$;

ii) Lamb pure directional weather types (e.g. N, S, or E-types) correspond to an essentially straight flow, when $|Z|$ is less than $F$;

iii) Lamb's pure cyclonic (C) and anticyclonic (A) types are identified when $|Z|$ is greater than $2 F$, respectively with $Z>0$ and $Z<0$;

iv) Lamb's hybrid types (e.g. AE and CSW) are characterised by a flow partially anticyclonic/cyclonic, with $|Z|$ lying between $F$ and $2 F$;

v) the unclassified (U) type is obtained when $F$ and $|Z|$ are less than 6 , with the choice of 6 depending on grid spacing.

The objective classification scheme yields 27 LWTs comprised of two synoptic types (A and C), five purely directional types (W, NW, E, N, and S), 19 hybrid combinations of synoptic and directional types (e.g. CNW, CSE and AE), and 1 unclassified (U) type (Jenkinson and Collison, 1977; Jones et al., 1993). For persistence and frequency analyses, the focus here is on the 7 synoptic and directional LWTs plus the U-type. Accordingly, counts of hybrid types were spread across the main types as per Lamb's original definition (Lamb, 1972, 1950) and common practice within earlier studies (Hulme et al., 1993; Jones et al., 2014, 2013, 1993). LWTs persistence and frequency are assessed for summer (June-July-August, JJA), autumn (September-October-November, SON), winter (December-JanuaryFebruary, DJF) and spring (March-April-May, MAM). On the other hand, when calculating indices of future multi-hazards, the hybrid LWTs were not incorporated into the 7 main types as the F-Score and nocturnal UHI indices require these weather patterns to be considered independently. 


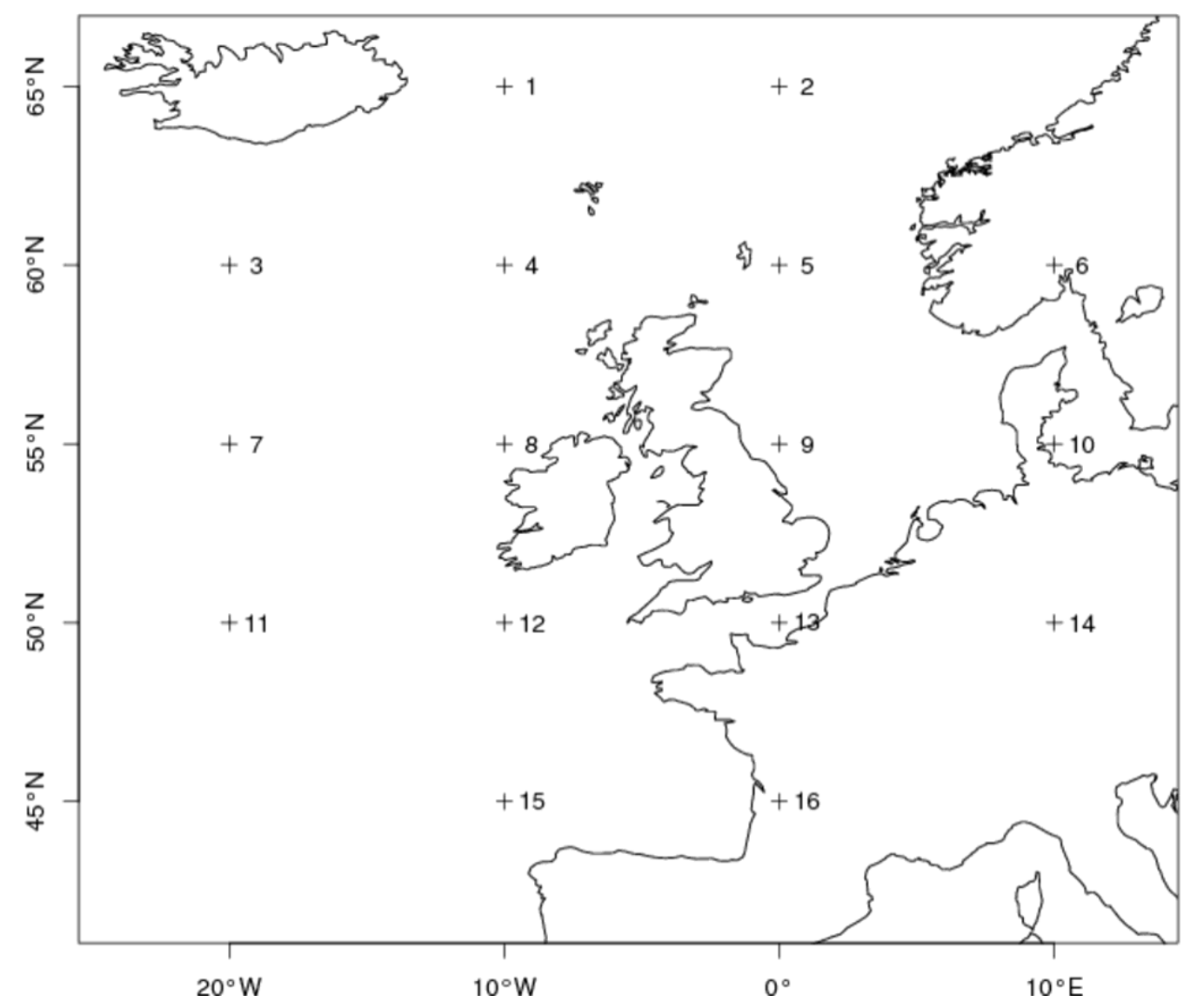

Figure 4.1 Grid points used to calculate Jenkinson flow and vorticity terms for the British Isles (BI). Numbers refer to those points used in Equations 4.1 to 4.5.

\subsubsection{Data}

Weather patterns were derived from the SLP produced by each AOGCM in the CMIP5 MME listed in Table 4.1 (Taylor et al., 2011). The historical period was defined as the 1980s (1971-2000) whereas the future was divided into three 30-year periods: the 2020s (2011-2040), 2050s (2041-2070) and 2080s (2071-2100). The CMIP5 AOGCMs and MMEM outputs for the historical period were compared with LWTs derived from 20CR (Compo et al., 2011), NCEP/NCAR (from now on defined as NCEP) (Kalnay et al., 1996) reanalyses and Lamb's subjective catalogue, which ends in 1997 (Hulme and Barrow, 1997; Lamb, 1972). The MMEM was built by first computing the LWTs and relative seasonal persistence and frequencies per each AOGCM then averaging these values within each time period. 


\begin{tabular}{|c|c|c|c|}
\hline Model name & Research institute & $\begin{array}{l}\text { Lat-Lon } \\
\text { resolution }\end{array}$ & $\begin{array}{l}\text { Ensemble } \\
\text { member }\end{array}$ \\
\hline HadGEM2-ES & Met Office, United Kingdom & $\begin{array}{l}1.25^{\circ} \times \\
1.875^{\circ}\end{array}$ & r1i1p1 \\
\hline MPI-ESM-LR & $\begin{array}{l}\text { Max Planck Institute for Meteorology, } \\
\text { Germany }\end{array}$ & $1.9^{\circ} \times 1.9^{\circ}$ & r1i1p1 \\
\hline MRI-CGCM3 & Meteorological Research Institute, Japan & $1.1^{\circ} \times 1.1^{\circ}$ & r1i1p1 \\
\hline CNRM-CM5 & $\begin{array}{c}\text { National Centre for Meteorological Research, } \\
\text { France }\end{array}$ & $1.4^{\circ} \times 1.4^{\circ}$ & r1i1p1 \\
\hline CanESM2 & $\begin{array}{c}\text { Canadian Center for Climate Modeling and } \\
\qquad \text { Analysis, Canada }\end{array}$ & $2.8^{\circ} \times 2.8^{\circ}$ & r1i1p1 \\
\hline MIROC5 & $\begin{array}{l}\text { Model for Interdisciplinary Research on } \\
\text { Climate, Japan }\end{array}$ & $1.4^{\circ} \times 1.4^{\circ}$ & r1i1p1 \\
\hline CSIRO-Mk3.6.0 & $\begin{array}{l}\text { Commonwealth Scientific and Industrial } \\
\text { Research Organisation, Australia }\end{array}$ & $1.9^{\circ} \times 1.9^{\circ}$ & r10i1p1 \\
\hline IPSL-CM5A-LR & Institute Pierre-Simon Laplace, France & $1.9^{\circ} \times 3.75^{\circ}$ & r1i1p1 \\
\hline CCSM4 & $\begin{array}{l}\text { National Center for Atmospheric Research, } \\
\qquad \text { USA }\end{array}$ & $\begin{array}{c}0.94^{\circ} \times \\
1.25^{\circ}\end{array}$ & r6i1p1 \\
\hline GFDL-CM3 & $\begin{array}{c}\text { Geophysical Fluid Dynamics Laboratory, } \\
\text { USA }\end{array}$ & $2^{\circ} \times 2.5^{\circ}$ & r1ilp1 \\
\hline
\end{tabular}

Table 4.1 CMIP5 multi-model sub-ensemble (MME) used in the analyses. The columns show the: (1) CMIP5 model name; (2) research institute where the model was developed; (3) resolution as latitude by longitude in degrees; and (4) ensemble member analysed. For all models the historical and RCP8.5 (and RCP4.5) sea-level pressure (SLP) outputs are used to calculate daily LWTs for the BI. 


\subsubsection{Persistence and trend analyses}

Weather pattern persistence is defined here as the conditional probability $\left(p_{j j}\right)$ that a given $\operatorname{LWT}_{\mathrm{j}}$ on $\operatorname{day}(t)$ is followed by the same $\operatorname{LWT}_{\mathrm{j}}$ on day $(t+1)$ (Gagniuc, 2017; Wilby, 1994). This diagnostic was extracted for the 7 main LWTs and the U-type using the diagonal cells of Markov-chain transition matrices. This enabled estimation of historical (1980s) and future (2020s, 2050s and 2080s) seasonal persistence for the MMEM as well as for individual AOGCMs for impactful weather types and seasons, the 20CR, NCEP reanalyses and Lamb's subjective catalogue.

Uncertainty in $p_{j j}$ for the 1980 s was calculated by boot-strapping $(n=1,000)$ 30-year seasonal simulations using the markovchain package within the R framework (Spedicato, 2017). This algorithm stochastically generates, with replacement, $n$ series of daily LWTs from the original conditional distributions of the weather patterns in each AOGCM, then recomputes $p_{j j}$ from each series. The resulting рвоотSTRAPjj is the mean of all $p_{j j}$ across the 1000 series, for each AOGCM. The 95\% confidence intervals of рвоотSTRAPjj are obtained from the cumulative distribution of the 1000 values of $p_{j j}$ for each AOGCM.

Statistical significance of changes in persistence for the AOGCM sub-ensemble between the 1980s and future periods was assessed using a Mann-Whitney-Wilcoxon two-tailed test (Mann and Whitney, 1947) applied to the 10 estimates of рвоотSTRAPjj for each time period. Changes in $p_{j j}$ between the 1980s and future periods for individual AOGCMs were regarded as statistically significant if future persistence of a given LWT and AOGCM fell outside the 95\% confidence intervals of the pвоOTSTRAPjj range of that AOGCM for the 1980s.

To detect both linear and non-linear annual changes in the total seasonal counts of LWTs MMEM frequencies under RCP8.5 and RCP4.5 scenarios, a trend analysis was performed for the 2006-2100 time period. For illustrative purposes, only trends for anticyclonic (A, summer JJA), cyclonic (C, autumn SON) and westerly (W, winter DJF) types, as indicators of impactful weather across the BI, are shown. Results are also presented for the southerly (S, spring MAM) types as this LWT shows most significant changes in seasonal persistence according to the non-parametric Mann-WhitneyWilcoxon two-tailed test between the 1980s and each of the three future periods (i.e. 2020s, 2050s and 2080s). A modified Mann-Kendall test, which takes into account possible autocorrelation within the time series, was applied to both RCP8.5 and RCP4.5 seasonal MMEM LWTs frequencies (Hamed and Ramachandra Rao, 1998). 


\subsubsection{Indices of winter flood-wind hazards and summer UHI intensity}

As a measure of concurrent flood-wind hazards an extended version of the F-Index (De Luca et al., 2017; Wilby and Quinn, 2013) is here calculated and defined as the F-Score, for each AOGCM, MMEM, NCEP, 20CR and Lamb's subjective datasets, covering the 1980s, 2020s, 2050s and 2080s, for selected LWTs known to drive these multi-hazard events (De Luca et al., 2017) during winter under both RCP8.5 and RCP4.5. The F-Index is the ratio of observed to expected frequency of floods for a given LWT, where values greater than 1 show higher than expected likelihood. Ten LWTs are known to be associated with historic, multi-basin floods (De Luca et al., 2017), of which eight (C, CS, CSW, CNW, S, SW, W, and NW-types) increase and two (N and A-types) reduce likelihood of multi-basin flood occurrence. All other LWTs are weighted zero. The F-Score is then calculated by multiplying the winter DJF frequencies $\left(f r e q_{-} d j f_{j, i}\right.$ ) of these LWTs by their $F_{-} I_{n d e x_{j, i}}$ (as per Event Set E in De Luca et al., 2017) and by summing these values:

$$
F_{-} \text {Score }_{i}=\sum_{j=1}^{10} \text { freq_djf }_{j, i} x F_{-} \text {Index } x_{j, i}
$$

where $i$ represents the single AOGCM, NCEP, 20CR and Lamb's subjective datasets within the relative time periods of 1980s, 2020s, 2050s, 2080s and $j$ is the given LWT considered from the 10 types mentioned above. The higher the F-Score, the greater the likelihood of concurrent multi-basin flood and wind hazards within winter, over the specified time horizon and RCP scenario.

As a proxy for combined heatwave and poor air quality hazards occurring during summer, observed, simulated and projected nocturnal UHI temperatures in tenths of degree Celsius for London (UK) are adopted (Wilby et al., 2011), using the same datasets, time periods and RCPs as per the F-Score. The UHI phenomenon is caused by absorption and trapping of heat as well as by changed airflows and sensible heat fluxes within the built environment. The simplest form of UHI metric (used by Wilby et al., 2011) is based on the daily temperature difference between an urban and rural reference site (during daylight or night hours). These values may then be stratified by LWT to show the extent to which some weather patterns favour extreme UHI episodes. The UHI metric was derived as follows by: i) multiplying LWT summer JJA frequencies $\left(\right.$ freq_jja $\left._{h, i}\right)$ by their respective average UHI intensities taken from Wilby et al. (2011) $\left(U H I_{-} w_{h, i}\right)$; ii) summing these values; and iii) dividing the total from step ii) by the total number of days in the period analysed $\left(\right.$ days $\left._{h, i}\right)$ to give the mean daily UHI intensity: 


$$
U H I_{i}=\sum_{h=1}^{27} \frac{\text { freq_}_{-} j j a_{h, i} x U H I_{-} w_{h, i}}{\operatorname{days}_{h, i}}
$$

where $i$ is the same notation as per the F-Score and $h$ refers to the 27 LWTs.

To assess the statistical significance of changes between the AOGCMs 1980s and future 2020s, 2050s and 2080s periods, for both the F-Score and nocturnal UHI temperatures, a similar approach as per persistence was applied. Here, $n=1,000$ boot-strapped samples of daily LWT series (based on conditional distributions for all seasons combined) were generated for each AOGCM run in the 1980s. Next, the F-Score or UHI were calculated for every series and AOGCM, then averaged and confidence limits established as before. This procedure shows the extent to which estimates for the future indices fall within the $95 \%$ confidence range of the boot-strapped estimate for each AOGCM in the 1980s.

Sample sizes varied depending on the index and AOGCM. For the F-Score, the period 1971-2001 was considered, to capture January and February of winter 2000/01. Here, models with leap years have a total of 11,323 days, models without leap years 11,315 days and the HadGEM2-ES model (with 360 days per year) has 11,160 days. For the UHI, the calendar years 1971-2000 were used as the interest here is summer temperatures, with leap year AOGCMs having 10,958 days, non-leap years models 10,950 days and the HadGEM2-ES 10,800 days. Further information about methods and data can be found in Annex 2 of this thesis.

\subsection{Results}

\subsubsection{Persistence of weather patterns (MME)}

The $\mathrm{A}, \mathrm{C}$ and $\mathrm{W}$ patterns are the most frequent weather types affecting the BI. Overall, the MME replicates weather type persistence during the four climatological seasons when compared with 20CR (Compo et al., 2011) and NCEP (Kalnay et al., 1996) reanalyses for the historical period (1980s) (Figure 4.2). There is less agreement between Lamb's subjectively classified daily weather catalogue and both the MME and reanalyses. A-type persistence is more variable within the MME and on average underestimated in winter, consistent with previous studies (Otero et al., 2018; Stryhal and Huth, 2018). There is closer agreement for the A-type in other seasons. 
W-type persistence agrees with the reanalyses but is always less than in Lamb's catalogue. C-type persistence is overestimated by the MME in all seasons when compared to reanalyses as reported before (Otero et al., 2018) for Europe more generally. Such biases in the C-type could be interpreted as exaggerating the likelihood of flooding in the MME compared with reanalyses (Wilby and Quinn, 2013).
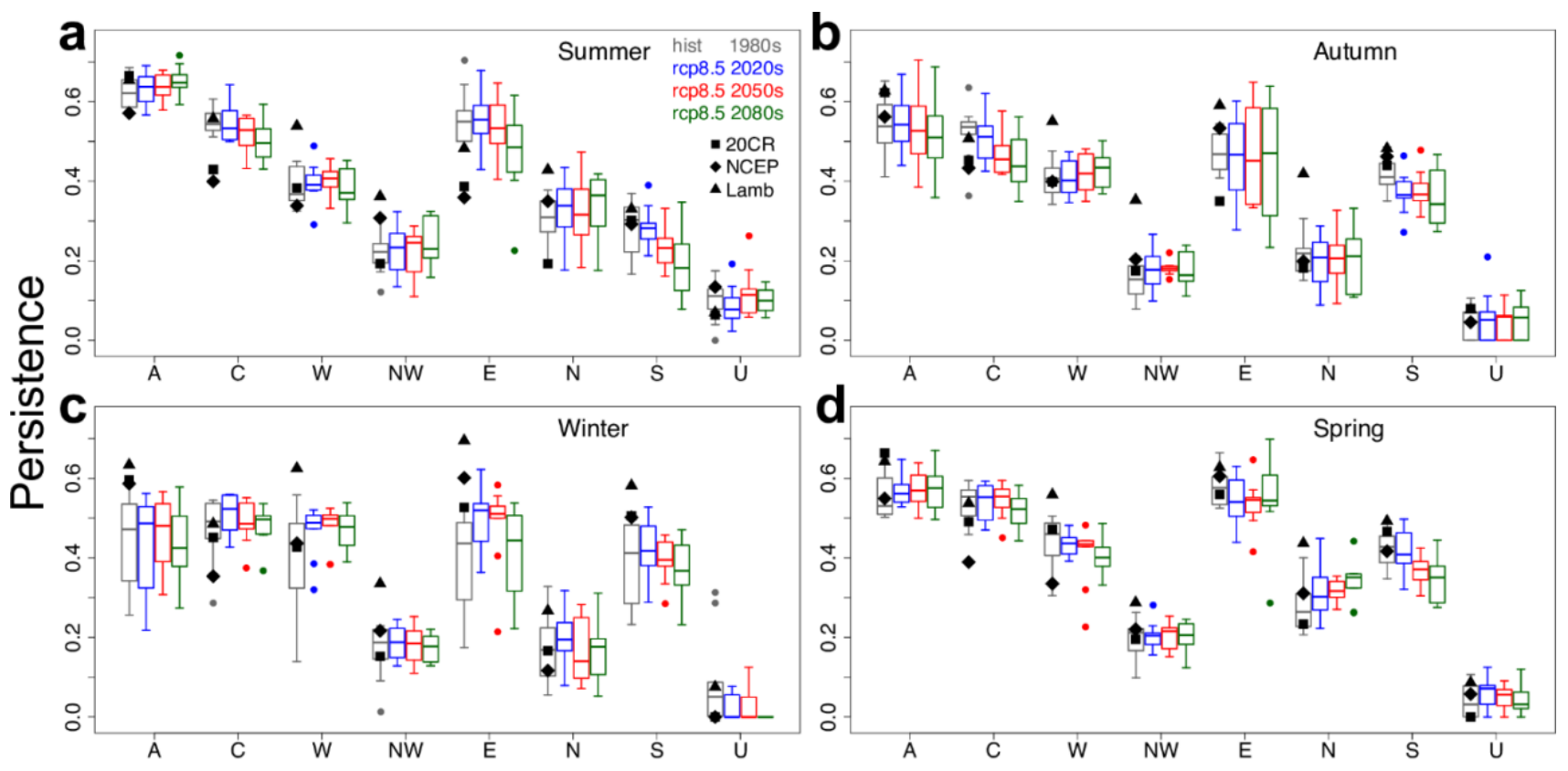

\section{Lamb Weather Types (LWTs)}

Figure 4.2 Persistence of the seven main LWTs plus unclassified (U) type under RCP8.5. Persistence is calculated for (a) summer, (b) autumn, (c) winter and (d) spring, for the historical 1980s period (1971-2000) and under RCP8.5 by the 2020s (2011-2040), 2050s (2041-2070) and 2080s (2071-2100). Boxplots show distributions of persistence in each LWT, for the 10-member AOGCM ensemble, compared with 20CR, NCEP and the Lamb's catalogue. Segments show the minimum, $1_{\text {st }}$ quartile, median, 3 rd quartile and maximum. Outliers are shown by dots.

Figure 4.2 shows that the distributions of persistence are asymmetrical (or skewed) around the MME means for many of the weather types and time periods. This characteristic suggests potentially large biases in the estimation of extreme events, if studies rely on only single or a few AOGCMs. Changes in weather type persistence between the ensembles of historical and future periods within RCP8.5 (Figure 4.2) are weakly significant (p-value<0.1, Mann-Whitney-Wilcoxon two-tailed test) for the Ctype in summer and autumn by 2080s; W-type in winter by 2050s; E-type in summer by 2080s and 
winter for the 2020s and 2050s; N-type in spring by 2050s and 2080s; and S-type in summer by 2080s, autumn in all periods and spring by 2050s and 2080s (Table 4.2).

\begin{tabular}{|c|c|c|c|c|c|c|c|c|}
\hline RCP8.5 & $\mathbf{1 9 8 0 s}$ & $\mathbf{1 9 8 0 s}$ & $\mathbf{1 9 8 0}$ & $\mathbf{1 9 8 0}$ & $\mathbf{1 9 8 0}$ & $\mathbf{1 9 8 0}$ & $\mathbf{1 9 8 0}$ & $\mathbf{1 9 8 0}$ \\
\hline JJA 2020s & 44 & 60 & 38 & 47 & 50 & 38 & 52 & $\mathbf{\text { C }}$ \\
\hline JJA 2050s & 42 & 65 & 40 & 44 & 54 & 44 & 68 & 43 \\
\hline JJA 2080s & 30 & $\mathbf{8 0}$ & 51 & 39 & $\mathbf{7 4}$ & 34 & $\mathbf{8 0}$ & 54 \\
\hline SON 2020s & 48 & 60 & 48 & 37 & 53 & 56 & $\mathbf{7 6}$ & 48 \\
\hline SON 2050s & 54 & 72 & 39 & 30 & 53 & 54 & $\mathbf{7 5}$ & 42 \\
\hline SON 2080s & 55 & $\mathbf{7 4}$ & 33 & 34 & 50 & 52 & $\mathbf{7 8}$ & 42 \\
\hline DJF 2020s & 55 & 38 & 29 & 43 & $\mathbf{2 4}$ & 34 & 40 & 71 \\
\hline DJF 2050s & 51 & 43 & $\mathbf{2 4}$ & 47 & $\mathbf{2 2}$ & 48 & 54 & 72 \\
\hline DJF 2080s & 58 & 52 & 29 & 54 & 43 & 44 & 61 & $\mathbf{9 0}$ \\
\hline MAM 2020s & 36 & 46 & 62 & 55 & 68 & 32 & 57 & 35 \\
\hline MAM 2050s & 39 & 49 & 64 & 47 & 72 & $\mathbf{2 3}$ & $\mathbf{8 6}$ & 40 \\
\hline MAM 2080s & 44 & 66 & 71 & 49 & 60 & $\mathbf{1 9}$ & $\mathbf{8 8}$ & 49 \\
\hline
\end{tabular}

Table 4.2 MME statistical significance of LWTs persistence for RCP8.5. Time periods considered are the 1980s compared to the 2020s, 2050s and 2080s under RCP8.5 during all seasons: summer JJA, autumn SON, winter DJF and spring MAM. Values shown are the W-statistic from the Mann-Whitney-Wilcoxon two-tailed test. Statistically significant values ( $p$-value $<0.1)$ are shown in bold.

Results for RCP4.5 show similar changes in persistence compared to RCP8.5, although they are less substantial (Figure 4.3). In particular, the C-type is found to change significantly (p-value <0.1) only in summer by the 2080s; the E-type in winter by the 2080s; the N-type only in spring by the 2080s; and the S-type in summer by the 2050s and spring also by the 2020s (Table 4.3). 

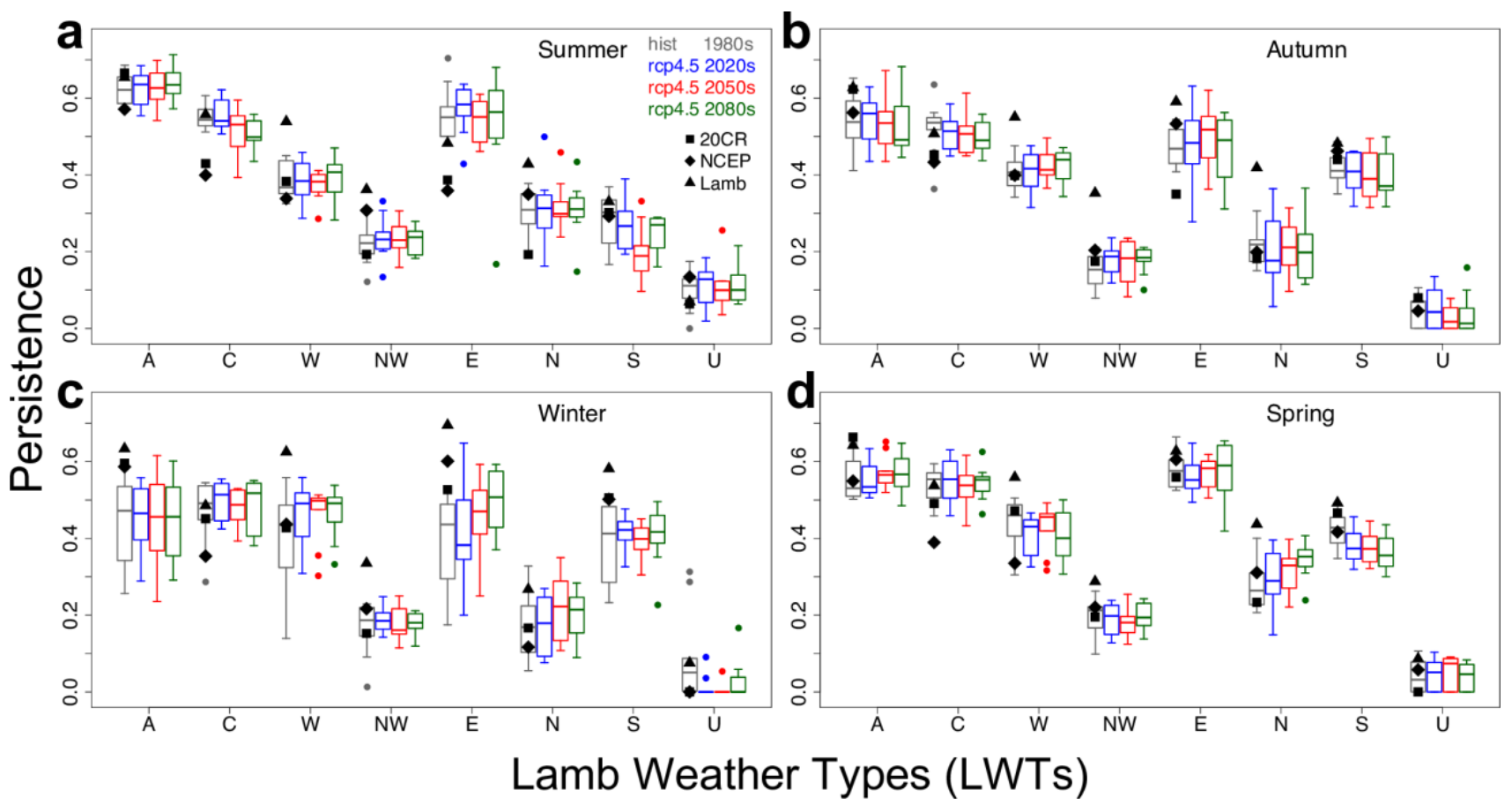

Figure 4.3 Persistence of the seven main LWTs plus unclassified (U) type under RCP4.5. Persistence is calculated for (a) summer, (b) autumn, (c) winter and (d) spring, for the historical 1980s period (1971-2000) and under RCP4.5 by the 2020s (2011-2040), 2050s (2041-2070) and 2080s (2071-2100). Boxplots show distributions of persistence in each LWT, for the 10-member AOGCM ensemble, compared with 20CR, NCEP and the Lamb's catalogue. Segments show the minimum, $1_{\text {st }}$ quartile, median, 3rd quartile and maximum. Outliers are shown by dots. 


\begin{tabular}{|c|c|c|c|c|c|c|c|c|}
\hline RCP4.5 & $\mathbf{1 9 8 0 s}$ & $\mathbf{1 9 8 0 s}$ & $\mathbf{1 9 8 0}$ & $\mathbf{1 9 8 0}$ & $\mathbf{1 9 8 0}$ & $\mathbf{1 9 8 0}$ & $\mathbf{1 9 8 0}$ & $\mathbf{1 9 8 0}$ \\
\hline JJA 2020s & 49 & 49 & 46 & 41 & 38 & 54 & 58 & 40 \\
\hline JJA 2050s & 44 & 64 & 51 & 45 & 54 & 47 & $\mathbf{8 2}$ & 52 \\
\hline JJA 2080s & 43 & $\mathbf{8 2}$ & 44 & 47 & 49 & 46 & 69 & 52 \\
\hline SON 2020s & 48 & 60 & 48 & 30 & 47 & 58 & 57 & 50 \\
\hline SON 2050s & 50 & 64 & 39 & 34 & 36 & 49 & 62 & 64 \\
\hline SON 2080s & 60 & 67 & 36 & 32 & 49 & 60 & 65 & 62 \\
\hline DJF 2020s & 53 & 41 & 33 & 47 & 49 & 44 & 47 & $\mathbf{8 0}$ \\
\hline DJF 2050s & 52 & 54 & $\mathbf{2 6}$ & 48 & 36 & 29 & 54 & $\mathbf{8 5}$ \\
\hline DJF 2080s & 50 & 43 & 31 & 52 & $\mathbf{2 2}$ & 34 & 48 & $\mathbf{7 5}$ \\
\hline MAM 2020s & 48 & 44 & 68 & 54 & 63 & 38 & $\mathbf{7 6}$ & 44 \\
\hline MAM 2050s & 36 & 56 & 57 & 64 & 54 & 31 & $\mathbf{8 0}$ & 41 \\
\hline MAM 2080s & 41 & 51 & 62 & 50 & 50 & $\mathbf{1 7}$ & $\mathbf{8 5}$ & 50 \\
\hline
\end{tabular}

Table 4.3 MME statistical significance of LWTs persistence for RCP4.5. Time periods considered are the 1980s compared to the 2020s, 2050s and 2080s under RCP4.5 during all seasons: summer JJA, autumn SON, winter DJF and spring MAM. Values shown are the W-statistic from the Mann-Whitney-Wilcoxon two-tailed test. Statistically significant values ( $p$-value <0.1) are shown in bold.

\subsubsection{Persistence of weather patterns (by model)}

Figure 4.4 shows persistence for the same future periods but for each AOGCM in the MME compared with the reanalyses and Lamb's catalogue, for impactful weather types and seasons. Significance of changes was assessed against the boot-strapped confidence limits for the 1980s. Most model projections under RCP8.5 fall outside the 95\% confidence intervals of historical persistence. A-type MMEM persistence increases during summer (Figures 4.2a and 4.4a); C-type persistence decreases in all seasons, most markedly in summer and autumn (Figures 4.2 and 4.4b); W-type persistence does not change in winter but increases in autumn and decreases in spring (Figures $4.2 \mathrm{~b}-\mathrm{d}$ and $4.4 \mathrm{c}$ ). 
Amongst the other weather types, only a decrease in C- and E-types during summer, an increase in Ntype in spring, and an S-type persistence decrease in all seasons can be noted (Figures 4.2 and 4.4d). The AOGCMs showing the largest change in A-type persistence during summer are CNRM-CM5, GFDL-CM3 and MIROC5, with a significant increase of 0.061, 0.059 and 0.035 respectively between 1980s and 2080s. For the C-type in autumn, CSIRO-Mk3.6.0, GFDL-CM3 and HadGEM2-ES show a significant decrease in persistence, between 1980s and 2080s, of 0.157, 0.140 and 0.098 respectively. During winter, for the W-type, the AOGCMs showing the largest change, between the same 1980s and 2080s periods, are MRI-CGCM3, CanESM2 and CSIRO-Mk3.6.0 with a significant increase in persistence of $0.367,0.334$ and 0.092 respectively.

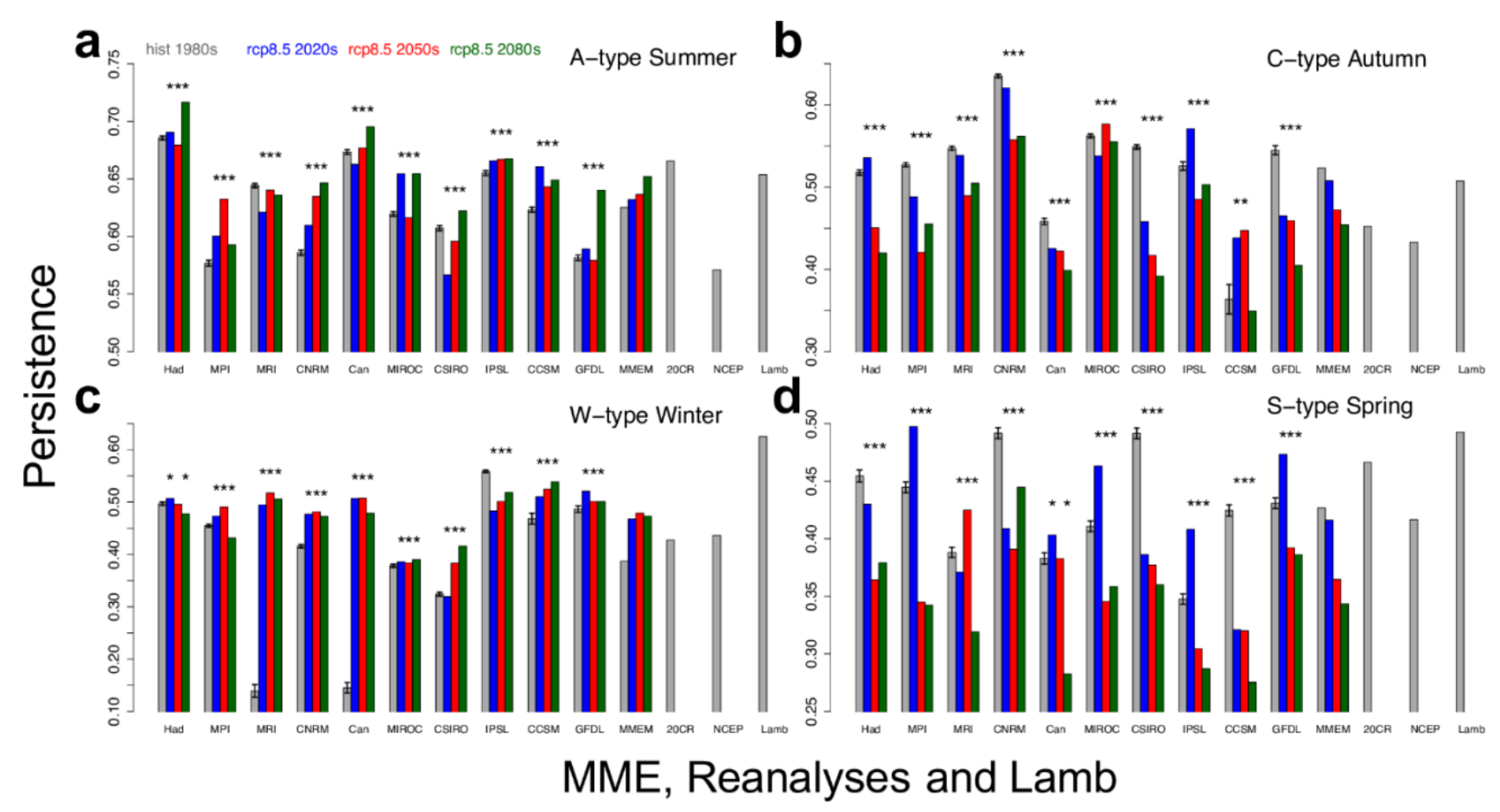

Figure 4.4 Persistence of selected LWTs and seasons for individual AOGCMs under RCP8.5. (a) A-type (summer), (b) C-type (autumn), (c) W-type (winter) and (d) S-type (spring) in the 1980s compared with the 2020s, 2050s and 2080s under RCP8.5. Persistence is shown for individual AOGCMs alongside the MMEM, 20CR, NCEP and Lamb's catalogue. Asterisks (*) show model runs with persistence outside the 95\% confidence intervals of the boot-strapped $(n=1,000)$ estimates for the 1980s, shown here as black T-bars.

Analysis of RCP4.5 output shows similar, though less marked, results when compared to RCP8.5 (Figure 4.5). Under the lower emission scenario, it is found that most AOGCMs project persistence that falls outside the $95 \%$ confidence intervals of the 1980s. A-type MMEM persistence in summer is 
expected to slightly increase, in particular during the 2080s (Figures 4.3a-4.5a), C-type in autumn may decrease (Figures 4.3b-4.5b), W-type during winter is projected to remain stable across the three future periods (Figures 4.3c-4.5c) and S-type persistence in spring decreases by 2100 (Figures 4.3d-4.5d). Other weather types changes in persistence are found for C-type in summer and A-type in autumn which are set to decrease and a marked increase in E-type during winter; the latter are not in agreement with RCP8.5 (Figure 4.3).

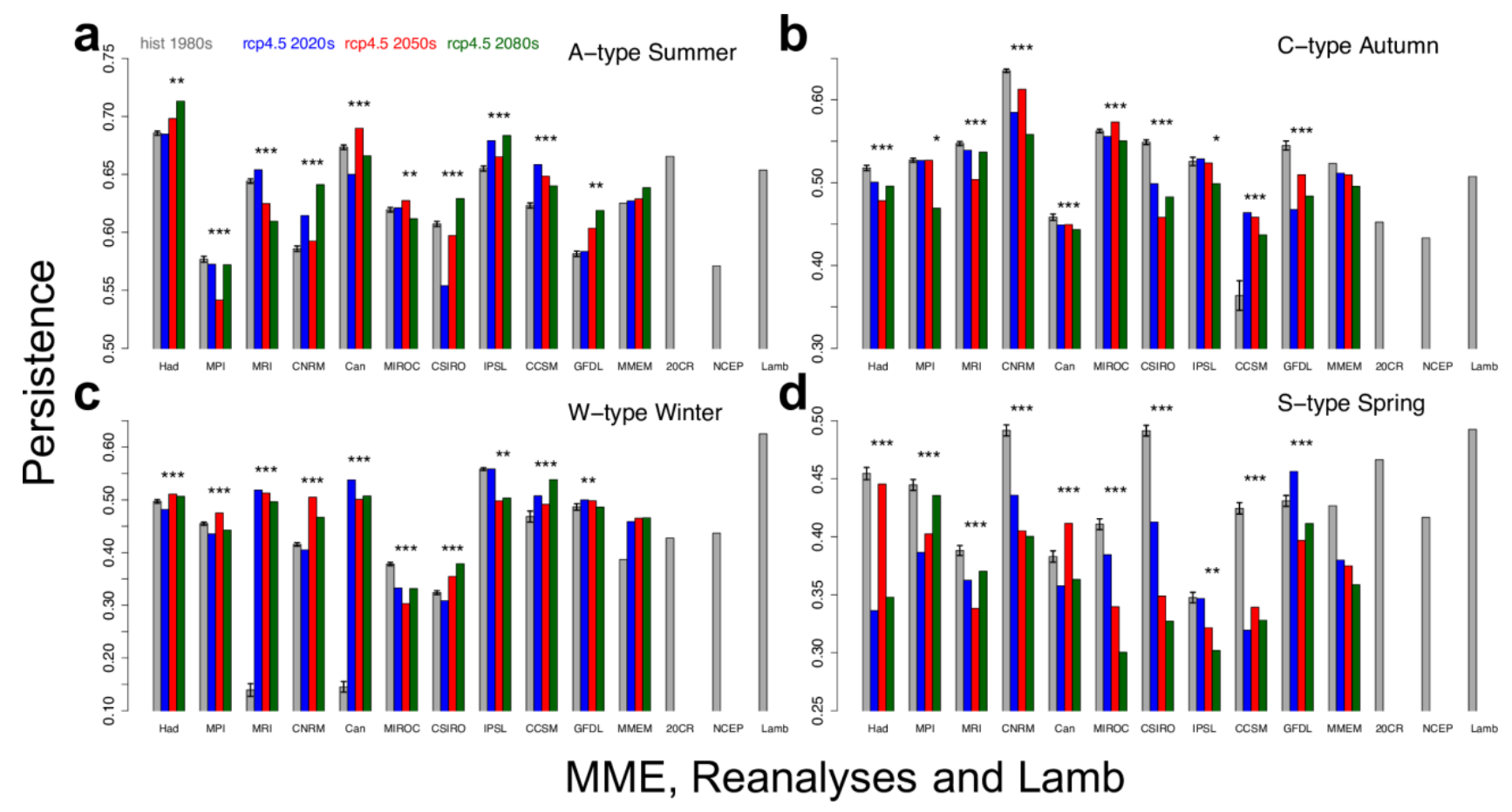

Figure 4.5 Persistence of selected LWTs and seasons for individual AOGCMs under RCP4.5. (a) A-type (summer), (b) C-type (autumn), (c) W-type (winter) and (d) S-type (spring) in the 1980s compared with the 2020s, 2050s and 2080s under RCP4.5. Persistence is shown for individual AOGCMs alongside the MMEM, 20CR, NCEP and Lamb's catalogue. Asterisks (*) show model runs with persistence outside the 95\% confidence intervals of the boot-strapped $(n=1,000)$ estimates for the 1980s, shown here as black T-bars.

\subsubsection{Frequency of weather patterns (MMEM)}

Projected frequency trends for selected weather types and seasons under RCP8.5 (2006-2100) are shown in Figure 4.6. Summer A- and winter W-type frequencies are expected to rise significantly (pvalue <0.01, Table 4.4) by 0.8 and 0.2 days per decade respectively over the period 2006-2100. Conversely, C- and S-type frequencies decrease significantly (p-value <0.01, Table 4.4) in autumn and spring respectively. No significant trends are found for C-type frequency during winter. Sen's slopes 
for the MMEM with their statistical significance are given in Table 4.4 for each weather type, season and RCP. The Sen's slopes for A-type in each individual AOGCM during summer was also computed (RCP8.5, not shown here), to check whether the increase in A-type was solely due to a few models showing a large increase in this weather type. It is found that all models within the MME show a positive increase in A-type frequency, with 7 out of 10 AOGCMs showing significance at the $90 \%$ level, with no outliers skewing the MMEM. Among other seasons (not shown), a significant decrease in annual frequencies is observed for the C-type during summer ( $\mathrm{p}$-value <0.01) and spring ( $\mathrm{p}$-value $<0.05$ ), along with a significant (p-value $<0.01$ ) increase in A-type during spring, which all reflect the changes in persistence (Figure $4.2 \mathrm{a}$ and $4.2 \mathrm{~d}$ ).

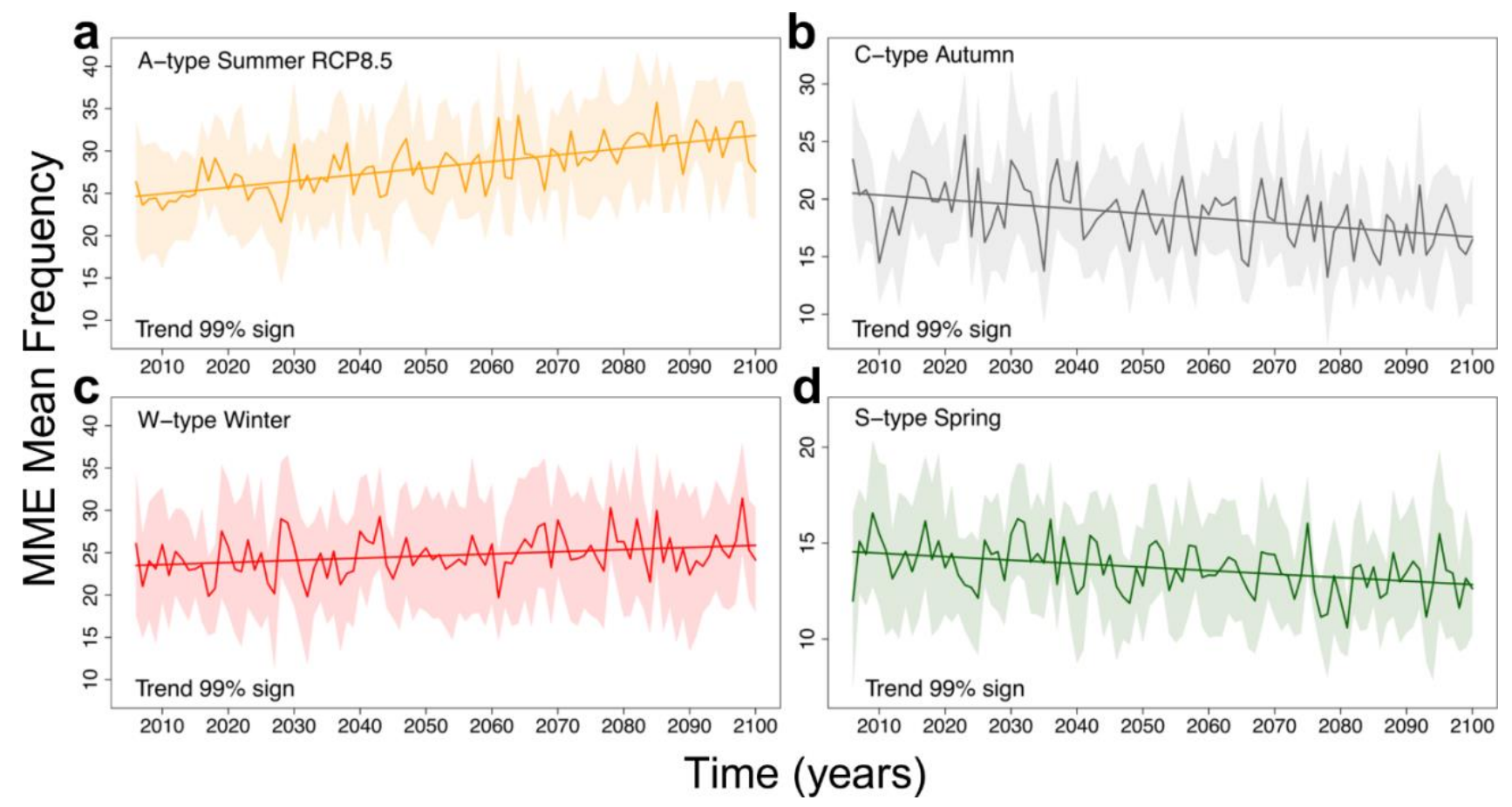

Figure 4.6 Projected annual frequencies for selected LWTs and seasons under RCP8.5. Frequencies are shown as MMEM for (a) summer anticyclonic A, (b) autumn cyclonic $C$, (c) winter westerly $W$ and $(d)$ spring southerly S LWTs under RCP8.5 (2006-2100). MMEM trends are statistically significant at the 1\% level (p-value <0.01, modified Mann-Kendall test). Shaded areas represent the 95\% confidence intervals of the MMEM. The trend lines refer to the Sen's slopes calculated with the modified Mann-Kendall test.

Projections of MMEM frequencies for the same LWTs and seasons but under RCP4.5 are shown in Figure 4.7 and Table 4.4. Results for RCP4.5 reflect the scenarios of RCP8.5 although the Sen's slopes are less extreme and statistically significant. The A-type frequency is projected to increase 
significantly (p-value $<0.01$, Figure $4.7 \mathrm{a}$ and Table 4.4) during summer, C-type in autumn is set to decrease (p-value $<0.05$, Figure $4.7 b$ ), W-type frequency in winter shows no significant trend (Figure 4.7c), and the S-type during spring decreases significantly (p-value $<0.05$, Figure 4.7d). As per RCP8.5, it is also observed (not shown) a significant decrease in C-type frequencies during summer (p-value <0.01) and spring ( $\mathrm{p}$-value $<0.05)$ and an increase in the A-type during spring ( $\mathrm{p}$-value <0.05), matching the relative changes in persistence (Figures $4.3 \mathrm{a}$ and $4.3 \mathrm{~d}$ ).

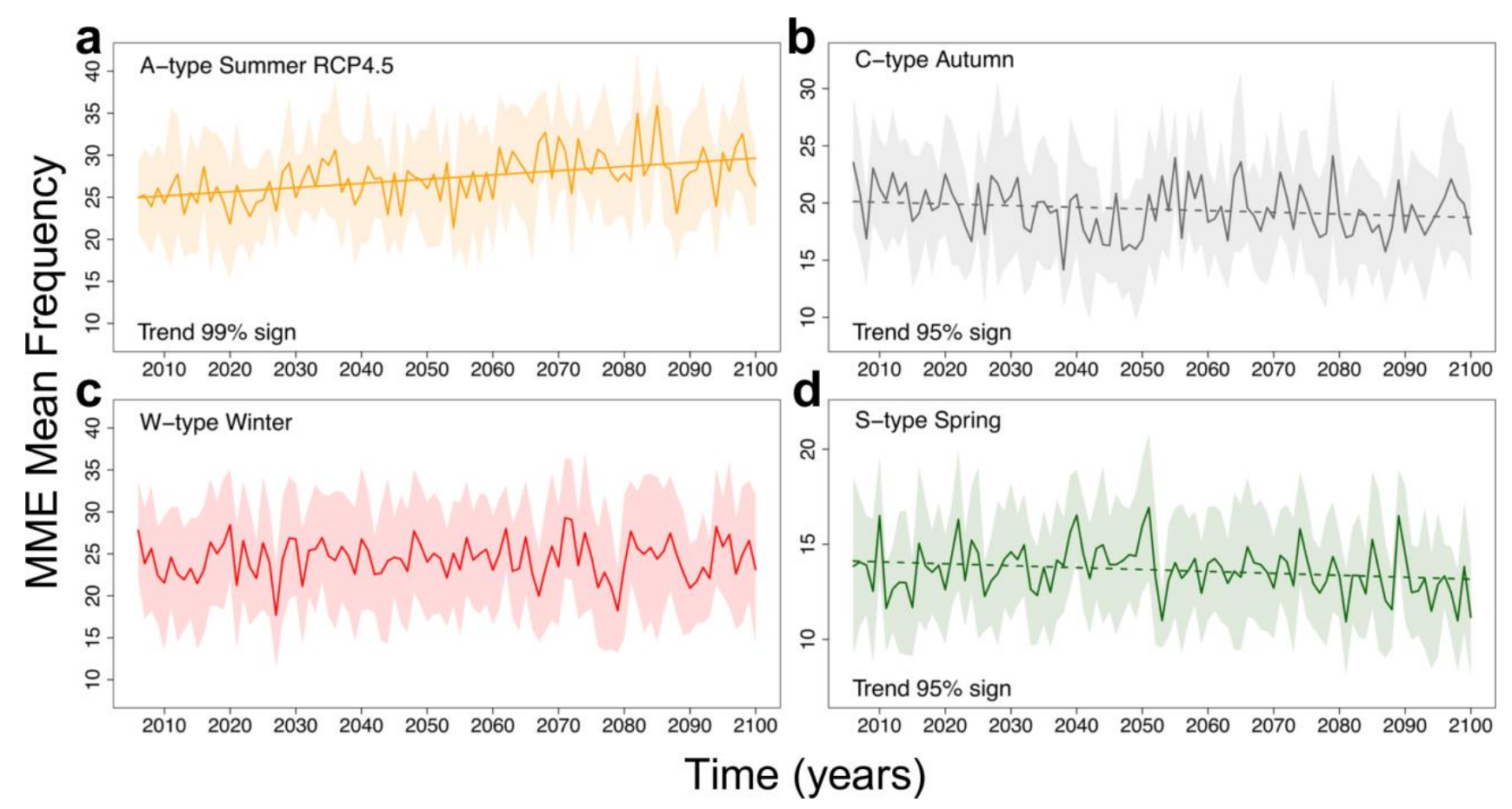

Figure 4.7 Projected annual frequencies for selected LWTs and seasons under RCP4.5. Frequencies are shown as MMEM for (a) summer anticyclonic A, (b) autumn cyclonic $C$, (c) winter westerly $W$ and $(d)$ spring southerly $S$ LWTs under RCP4.5 (2006-2100). MMEM trends are statistically significant at the $1 \%$ and 5\% levels (pvalue $<0.01$ and $<0.05$, modified Mann-Kendall test). Shaded areas represent the $95 \%$ confidence intervals of the MMEM. The trend lines refer to the Sen's slopes calculated with the modified Mann-Kendall test. 


\begin{tabular}{|l|l|l|}
\hline \multicolumn{2}{|c|}{} & Sen's slopes \\
\hline \multirow{2}{*}{$\begin{array}{l}\text { Summer JJA } \\
\text { (A-type) }\end{array}$} & RCP8.5 & $8.04 \mathrm{e}-02 * *$ \\
\cline { 2 - 3 } Autumn SON & RCP4.5 & $4.71 \mathrm{e}-02 * *$ \\
\cline { 2 - 3 } (C-type) & RCP8.5 & $-4.17 \mathrm{e}-02 * *$ \\
\hline \multirow{2}{*}{$\begin{array}{l}\text { Winter DJF } \\
\text { (W-type) }\end{array}$} & RCP4.5 & $-1.71 \mathrm{e}-02 *$ \\
\cline { 2 - 3 } & $\mathbf{R C P 4 . 5}$ & $4.17 \mathrm{e}-03$ \\
\hline \multirow{2}{*}{$\begin{array}{l}\text { Spring MAM } \\
\text { (S-type) }\end{array}$} & $\mathbf{R C P 8 . 5}$ & $-1.88 \mathrm{e}-02 * *$ \\
\cline { 2 - 3 } & $\mathbf{R C P 4 . 5}$ & $-9.93 \mathrm{e}-03 *$ \\
\hline
\end{tabular}

Table 4.4 Sen's slopes of MMEM seasonal LWTs frequencies for RCP8.5 and RCP4.5. The slopes are calculated using a modified Mann-Kendall trend test over the 2006-2100 period. Four LWTs are shown: anticyclonic (A) for summer JJA; cyclonic $(C)$ autumn SON; westerly $(W)$ winter DJF and southerly $(S)$ spring MAM. MMEM statistical significance is shown as *p-value $<0.05$ and $* *$-value $<0.01$.

\subsubsection{Application to future multi-hazards}

In Figure 4.8 an earlier analysis (De Luca et al., 2017) based on impactful LWTs found to generate concurrent flood-wind hazards in GB is extended. Thus, the F-Score for each single AOGCM, MMEM, NCEP, 20CR and Lamb's subjective datasets and 1980s, 2020s, 2050s and 2080s time periods are shown for winter DJF weather patterns under RCP8.5. The F-Score is a measure of the severity of future concurrent flood-wind hazards, such that higher values represent more severe impacts compared to lower ones. Here, it is found that the baseline risk from multiple flood-wind hazards is overestimated by all but two of the AOGCMs (i.e. HadGEM2-ES and MIROC5) when compared to NCEP, 20CR reanalyses and Lamb's subjective catalogue for the 1980s. Assuming the same bias holds in the future, AOGCMs likely overestimate absolute future risk from concurrent floodwind hazards by 2100 . Moreover, in a similar way as per Figure 4.4, there exists a large variability between the AOGCMs, so F-Score results are mixed with some AOGCMs suggesting increased/decreased risk of flood-wind hazards by the end of the $21_{\text {st }}$ century. It is, therefore, always important to use large ensembles to characterise uncertainty in the projections. Lastly, by looking at the MMEM it is possible to conclude that, although overestimated by AOGCMs, future risk from concurrent flood-wind hazards could increase by 2100 compared with the 1980s. Among the 
AOGCMs, those showing the largest F-Score increase between the 1980s and 2080s are CanESM2, CCSM4 and IPSL-CM5A-LR. Results for RCP4.5 are shown in Figure 4.9 and they agree with what was found for RCP8.5, with large variability amongst AOGCMs and MMEM F-Score even slightly higher than RCP8.5.

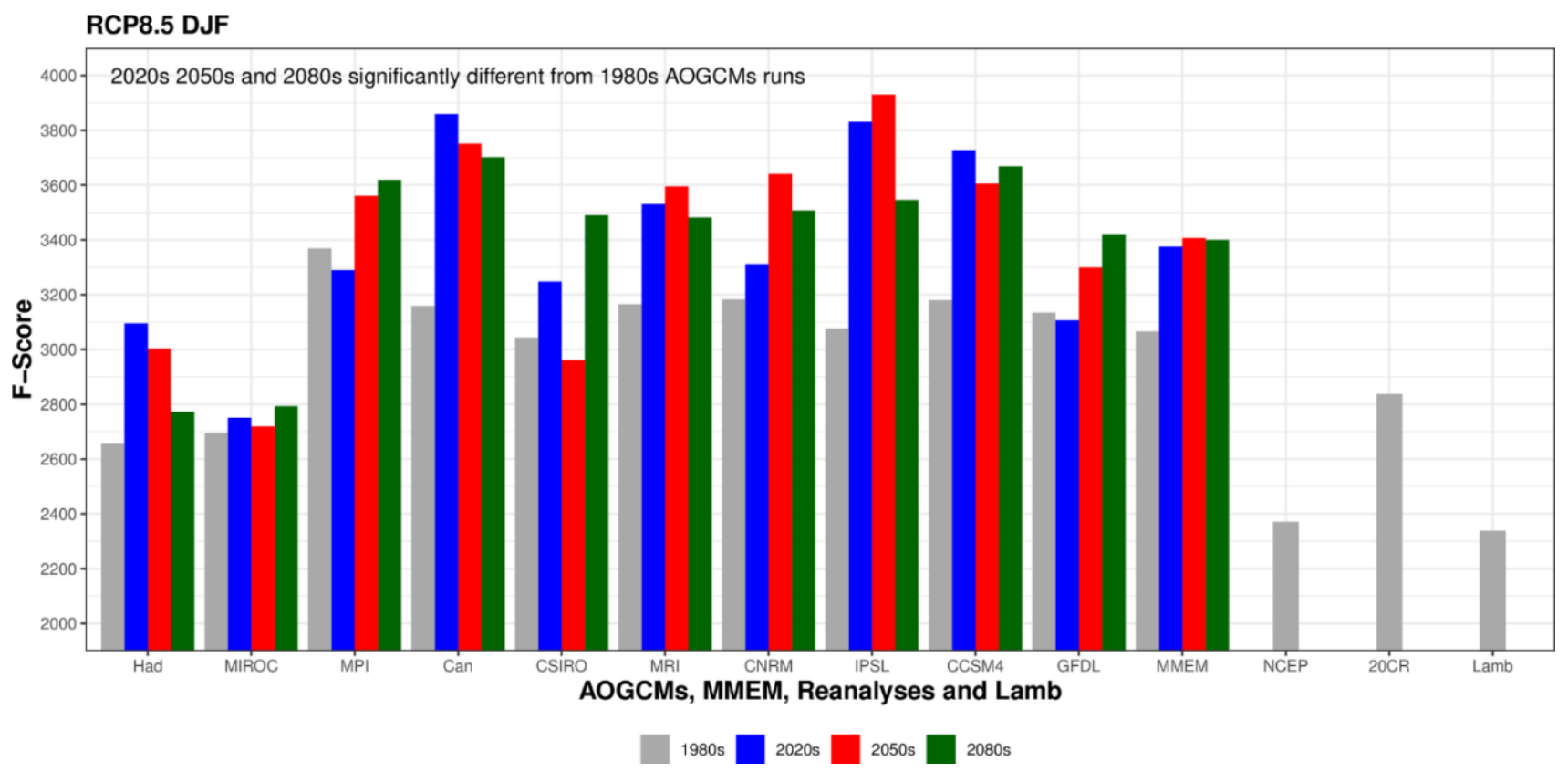

Figure 4.8 F-Score for LWTs associated with concurrent flood-wind hazards during winter DJF. The F-Score is shown per each CMIP5 AOGCM, MMEM, NCEP, 20CR and Lamb's subjective catalogue for the 1980s, 2020s, 2050s and 2080s periods under RCP8.5. The LWTs used for calculating the F-Score are associated with concurrent multi-basin floods and wind hazards within Great Britain (GB) (De Luca et al., 2017). The 1980s MME F-Score were estimated from the mean of $n=1,000$ boot-strapped samples. The AOGCMs 1980s confidence intervals bars are not shown for simplicity because they are vanishingly narrow. 


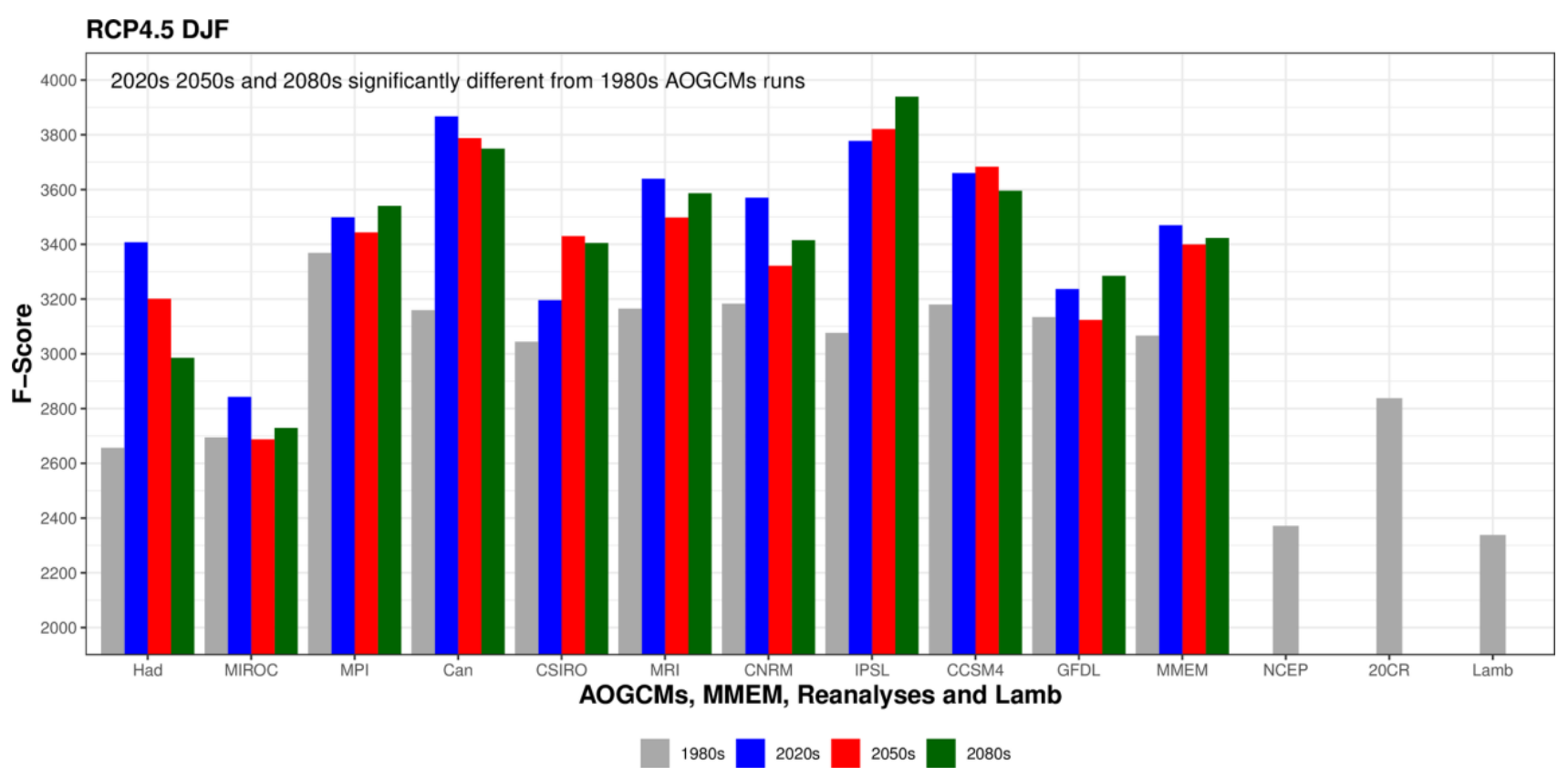

Figure 4.9 F-Score for LWTs associated with concurrent flood-wind hazards during winter DJF. The F-Score is shown per each CMIP5 AOGCM, MMEM, NCEP, 20CR and Lamb's subjective catalogue for the 1980s, 2020s, 2050s and 2080s periods under RCP4.5. The LWTs used for calculating the F-Score are associated with concurrent multi-basin floods and wind hazards within Great Britain (GB) (De Luca et al., 2017). The 1980s MME F-Score were estimated from the mean of $n=1,000$ boot-strapped samples. The AOGCMs 1980s confidence intervals bars are not shown for simplicity because they are vanishingly narrow.

Summer nocturnal UHI temperatures in tenths of ${ }^{\circ} \mathrm{C}$ for London (UK), were estimated for RCP8.5, by using UHI values obtained in a previous study (Wilby et al., 2011) (Figure 4.10). These results show that AOGCMs replicate nocturnal UHI temperatures, although there is a tendency for underestimation by the majority of AOGCMs except HadGEM2-ES and MIROC5 which show a good agreement when compared to NCEP, 20CR and Lamb's subjective catalogue. It is also worth noting that there is less variability within the MME than displayed in Figures 4.4 and 4.8. Lastly, almost all the AOGCMs and MMEM show a statistically significant increase in UHI by the end of 2100 , that could eventually translate into an increased multi-hazard risk from heatwave and poor air quality events associated with persistent A weather types (O'Hare and Wilby, 1995; Pope et al., 2016, 2014; Wilby et al., 2011). The projected increase in the MMEM UHI between the $1980 \mathrm{~s}$ and $2080 \mathrm{~s}$ is $0.15^{\circ} \mathrm{C}$ under RCP8.5. The AOGCMs that show the largest increase in nocturnal UHI temperatures between 1980s and 2080s are CanESM2, HadGEM2-ES and CCSM4 with respectively $0.23,0.22$ and $0.22{ }^{\circ} \mathrm{C}$. Results for RCP4.5 agree with the RCP8.5 projections although the changes are less marked (Figure 4.11). 


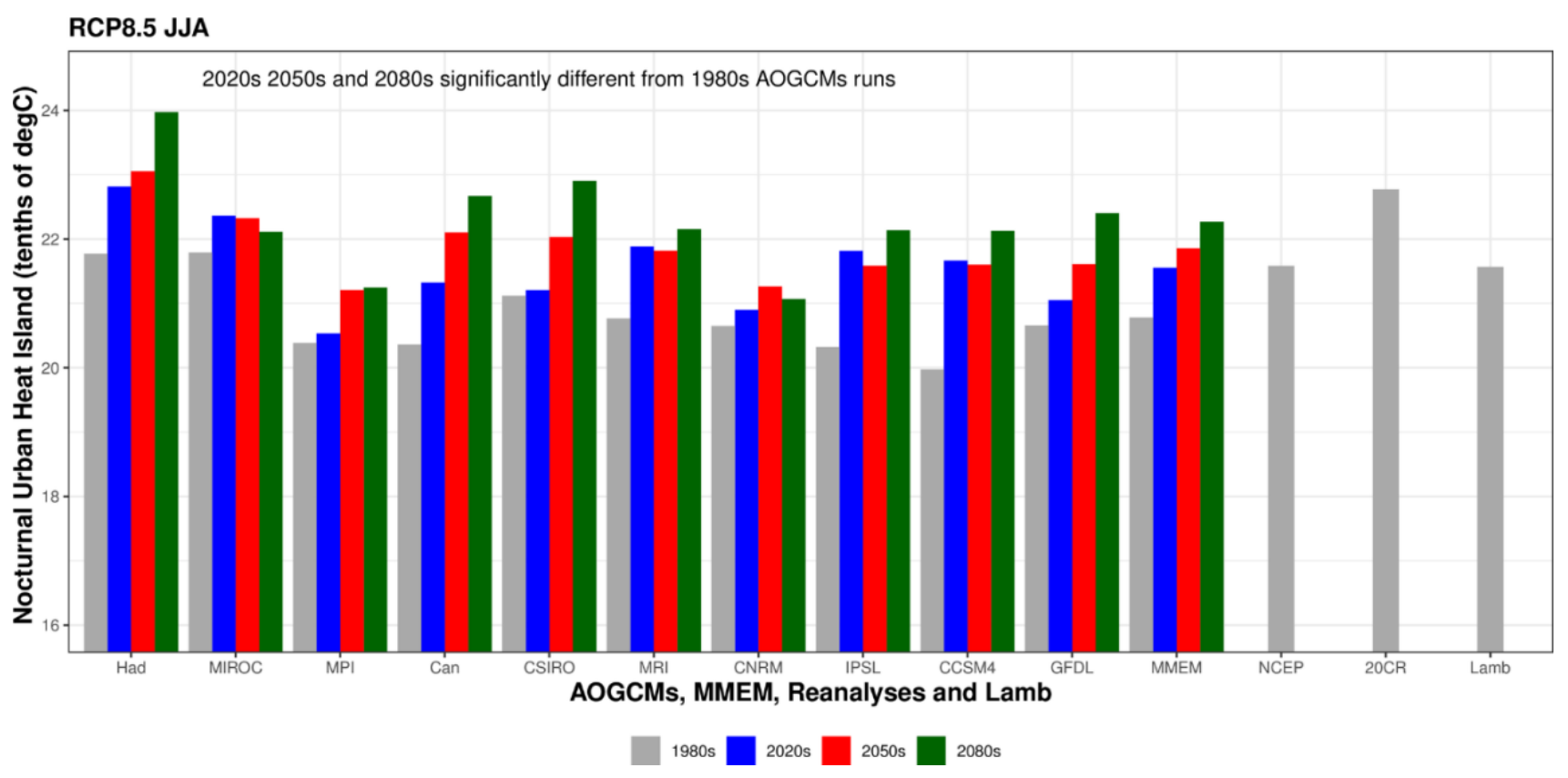

Figure 4.10 UHI in tenths of ${ }^{\circ} \mathrm{C}$ for LWTs associated with concurrent heat-air pollution hazards during summer JJA. The UHI is shown per each CMIP5 AOGCM, MMEM, NCEP, 20CR and Lamb's subjective catalogue for the 1980s, 2020s, 2050s and 2080s periods under RCP8.5. The 1980s MME UHI were estimated from the mean of $n=1,000$ boot-strapped samples. The AOGCMs 1980 s confidence intervals bars are not shown for simplicity because they are vanishingly narrow. 


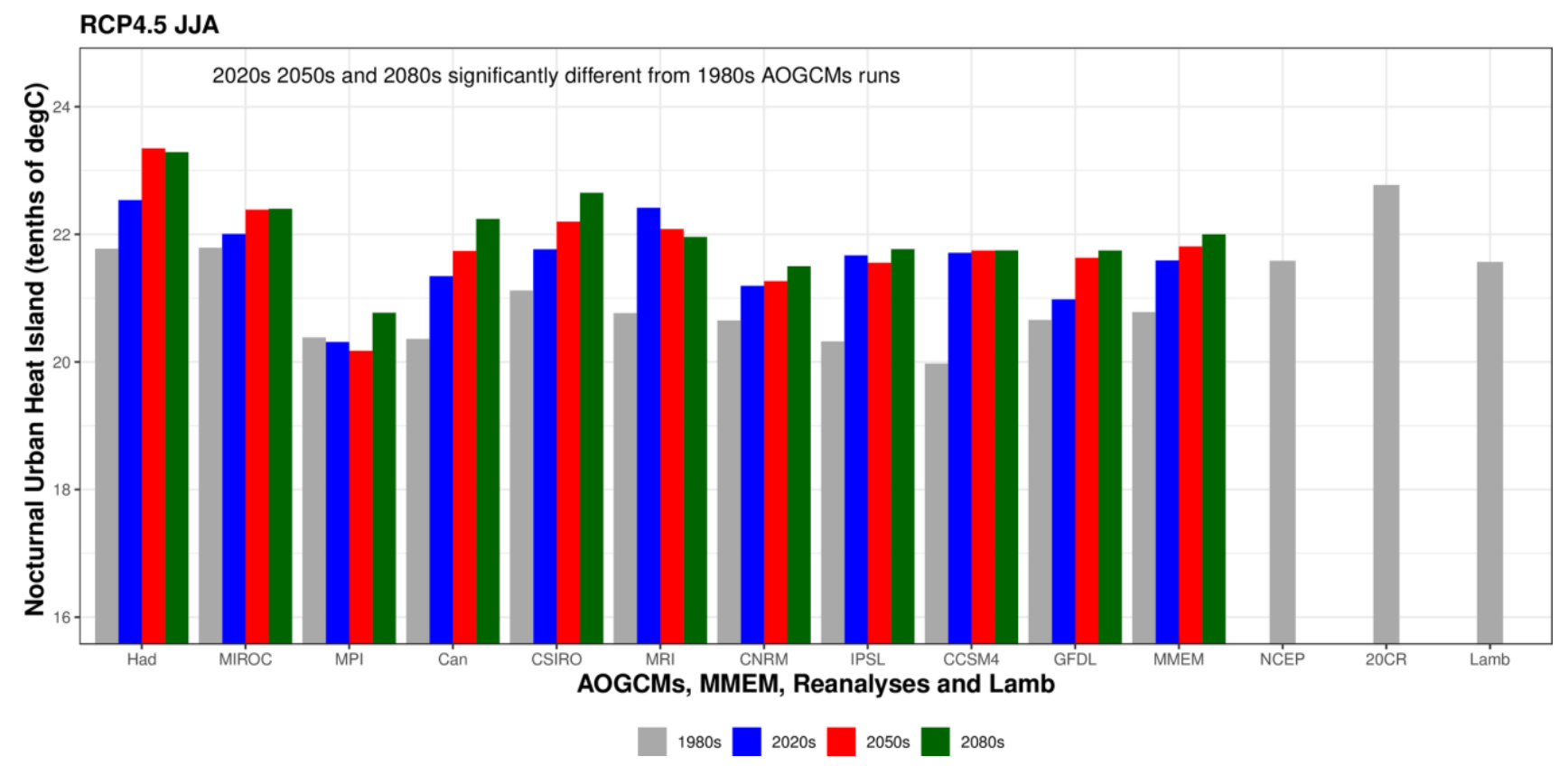

Figure 4.11 UHI in tenths of ${ }^{\circ} \mathrm{C}$ for LWTs associated with concurrent heat-air pollution hazards during summer JJA. The UHI is shown per each CMIP5 AOGCM, MMEM, NCEP, 20CR and Lamb's subjective catalogue for the 1980s, 2020s, 2050s and 2080s periods under RCP4.5. The 1980s MME UHI were estimated from the mean of $n=1,000$ boot-strapped samples. The AOGCMs 1980s confidence intervals bars are not shown for simplicity because they are vanishingly narrow.

\subsection{Discussion and Conclusions}

Answers to research questions 1-4, along with discussion, can be found in the following paragraph. For research questions 1-3, the focus is here concentrated first on Anticyclonic (A), then Cyclonic (C) and finally Westerly (W) weather patterns in three subsections. Whereas research question 4 finds its answer and discussion in two subsections, the first focussing on winter flood-wind hazards and the second on summer heatwave-air pollution hazards. The last two subsection of the paragraph briefly discuss the limitations and usefulness of the approach used.

Greater A-type persistence and frequency during summer likely implies more blocking episodes with increased risk of poor air quality, drought and heatwaves (Coumou et al., 2018; Munich Re, 2015; Tang et al., 2013). A growing number of studies propose physical mechanisms that link AA (Screen and Simmonds, 2010) to more persistent weather patterns, which in turn enhance the likelihood of extreme weather events in the northern hemisphere mid-latitudes. The AA affects the polar jet stream by making Rossby waves more meridional (or wavier) and by weakening its flow. A wavier and weaker jet stream in summer favours more persistent extreme weather and it is also thought to extend 
ridges northward, enhancing such effects (Cohen et al., 2014; Coumou et al., 2018; Francis and Skific, 2015; Francis, 2017; Francis et al., 2017; Francis and Vavrus, 2012; Tang et al., 2013). Diverse studies shown that the jet stream, since 1979, has become wavier (e.g. Coumou et al., 2018; Di Capua and Coumou, 2016).

The results found in Chapter 4 support earlier analysis (Otero et al., 2018), and are consistent with the proposed mechanisms linking observed AA with mid-latitude weather extremes. On the other hand, AA is expected to have limited effect on simulated CMIP5 blocking over Eurasia under RCP8.5 in the second half of the 21 st century (Woollings et al., 2014). Other work also shows an overall decrease in CMIP5 blocking events over the BI in winter DJF and summer JJA, during 2061-2090 (RCP8.5) (Woollings et al., 2018). The findings for anticyclonic weather appear to contradict this. Although Atype persistence and frequency are not the same as blocking, there was an expectation that the studies to agree as both mechanisms involve high pressure weather patterns. The only common denominator between the findings and the studies on blocking (Woollings et al., 2018, 2014) seems to be the underestimation of A-type/blocking events by CMIP5 models. Further research is needed to reconcile these apparently contradictory findings. Possible explanations are that results depend on the exact spatial domain and/or suite of AOGCMs analysed in each MME, as well as on the methodology used to define A-type days and blocking events.

Less persistent C-types in autumn suggests lower likelihood of heavy rainfall, with reduced recharge of soil moisture and aquifers at the start of the hydrological year favouring winter droughts. Fewer cyclonic days may also translate into less frequent severe gales and flooding episodes (Wilby and Quinn, 2013), as in GB extreme multi-basin flooding events are strongly associated with C-type weather over time windows from 1 to 19 days (De Luca et al., 2017). Conversely, more frequent zonal airflow (W-type) in winter may counteract some loss of precipitation from the C-type, especially across higher elevation regions of the north and west BI where there is strong orographic enhancement (Burt and Howden, 2013). Such changes may be attributed to AA, however, the physical mechanisms linking AA to changes in northern hemisphere mid-latitude circulation currently remains an open question.

From the analyses it is also possible to infer future changes with respect to multi-hazards (Gill and Malamud, 2014; Zscheischler et al., 2018), through the F-Score and nocturnal UHI temperatures (research question 4). In Chapter 3 it is shown that in GB multi-basin flooding and extreme wind events are driven by LWTs mainly associated with C- and W-types (De Luca et al., 2017). These multi-hazard 
events can generate significant economic losses, hence projections of such events may help in evaluating future risks and in improving resilience. It is shown that during winter DJF the AOGCMs overestimate the F-Score when compared to NCEP, 20CR reanalyses and Lamb's subjective dataset. Even so, by the end of 2100 the MMEM shows a statistically significant increase in the F-Score compared with the 1980s within those same models, suggesting that the risk of concurrent flood-wind impacts may become more severe in a warmer world.

Nocturnal UHI temperatures in London modelled by AOGCMs agree with NCEP, 20CR and Lamb's subjective datasets, although they are slightly underestimated for the 1980s. Nocturnal UHI severity is expected to increase by 2100 under RCP8.5 (MMEM). The results confirm an increasing trend of $\sim 0.3{ }^{\circ} \mathrm{C}$ in nocturnal UHI in London found in an earlier study over the observational period 1950-2006 (Wilby et al., 2011). The findings are also in line with the UK Climate Projections Science Report 2009 (Murphy et al., 2009) which suggests that intense UHI events are highly correlated with A-type weather patterns, and that in London, intense UHI summer events are expected to become more severe in the future (Wilby, 2008). However, further analysis of projections of UHI is needed with a larger model ensemble to better account for uncertainty. These present findings, when coupled with a significant increase in persistence and frequency of A-type weather pattern, suggests a combined increased risk of heatwaves and poor air quality events in London (O'Hare and Wilby, 1995; Pope et al., 2016, 2014; Wilby, 2008; Wilby et al., 2011) that could negatively impact human health.

Finally, it is illustrated how changes in the persistence and frequency of weather patterns are useful diagnostics of climate model realism and can translate into regional to local weather and climate risks scenarios, which could be helpful for developing narratives for decision-makers. However, caution needs to be taken when qualitatively converting synoptic weather pattern changes into local variability because AOGCM skill in reproducing climatic variables at local scales varies significantly and is not always consistent with observations. This is particularly true for precipitation where, for example, pressure fields alone are not able to provide reliable local projections (Murawski et al., 2016).

With the UK Climate Projections 2018 partly released and the third UK Climate Change Risk Assessment now underway, weather pattern analysis could help to both evaluate the new projections and offer ways of explaining changes that are intelligible to a range of user communities. Similar links to persistence could be made in other regions with established weather pattern typologies, such as the Grosswetterlagen for Europe (Hess and Brezowsky, 1952), hydrologically important weather types in 
the contiguous United States (Prein et al., 2019) and Spatial Synoptic Classification for North America (Kalkstein et al., 1996).

\subsection{Summary}

In the above chapter, an investigation of observed, simulated and projected weather patterns (or Lamb Weather Types, LWTs) (Jones et al., 1993; Lamb, 1972) over the British Isles (BI) is presented (De Luca et al., 2019a). Specifically, the focus was on the quantification of LWTs frequency and persistence, by evaluating how well the latter is simulated by a sub-ensemble of CMIP5 models (Taylor et al., 2011) and how this might change in the future under two RCP scenarios (RCPs 8.5 and 4.5). Furthermore, associated multi-hazards, driven by LWTs, were also addressed from both the quantitative and qualitative perspectives. Results show that CMIP5 models are generally able to reproduce LWTs persistence when compared to NCEP and 20CR reanalyses, with the anticyclonic (A) and cyclonic (C) weather types respectively underestimated and overestimated. The A-types are set to increase by 2100 , during summer, in both frequency and persistence. The C-types, on the other hand, may decrease in the future during autumn. This translates into an enhanced (decreased) risk of heatwave, drought and air pollution (flooding and storm) events. With respect to multi-hazards, the computation of the F-Score, an index that quantifies the impacts of concurrent flood and storms events, shows that the CMIP5 models overestimate it during winter, but nonetheless the index is set to increase in the future. The summer nocturnal Urban Heat Island (UHI) temperatures for London (UK) were also computed (Wilby, 2008; Wilby et al., 2011) and results show that the UHI is slightly underestimated by CMIP5 models and expected to intensify by 2100 . These results could signify an enhanced risk of concurrent flood-storm and heatwave-air pollution events respectively during winter and summer by 2100 . The analyses were performed for the British Isles and London, however, they could also be applied in other regions and cities within the mid-latitudes and beyond.

In the next Chapter the geographical region under consideration is extended further and a diverse set of concurrent hydroclimatic hazards are analysed. Hence, Chapter 5 quantifies concurrent wet and dry hydrological extremes at the global scale and link these to modes of climate variability (De Luca et al., 2019c). The analysis is based on the Palmer Drought Severity Index (PDSI) (Dai et al., 2004; Palmer, 1965) over the 1950-2014 period and climate indices used are the El Niño-Southern Oscillation (ENSO) (Rayner et al., 2003; Trenberth, 1997), Pacific Decadal Oscillation (PDO) (Mantua and Hare, 2002) and American Multi-decadal Oscillation (AMO) (Schlesinger and 
Ramankutty, 1994). Similarly as per Chapters 3-4, Chapter 5 introduces new metrics specifically used for assessing spatio-temporal joint occurrences of hydroclimatic hazards. Furthermore, Chapter 5 shows the most impactful (i.e. geographically widespread) concurrent flood and drought events, and it lays the bases for future climate modelling studies, similar to the ones presented in Chapter 4. 


\section{Chapter 5 \\ Concurrent wet and dry hydrological extremes \\ at the global scale}

\subsection{Introduction}

As mentioned in previous Chapters, hazards can interact in diverse ways, leading to multi-hazard events that can exacerbate disaster losses when compared to single-hazard occurrences (Zscheischler et al., 2018). Such combination of perils can lead to situations beyond the worst-case scenario planned by emergency managers, (re)insurance companies, businesses and governments. Thus, multi-hazard events present a critical challenge for disaster risk reduction, as demonstrated for example by the 2010 Russian heatwave (Barriopedro et al., 2011; Zscheischler et al., 2018). The relevance of multi-hazards has been recognised by both the scientific and stakeholder communities - both have devoted significant efforts to the topic over the past decade, achieving a significant review with linked socioeconomic implications of multi-hazard events (e.g. Forzieri et al., 2016; Gallina et al., 2016; Gill and Malamud, 2014; Kappes et al., 2012a; Leonard et al., 2014; Terzi et al., 2019; Tilloy et al., 2019; UNDRR, 2015; Zscheischler et al., 2018).

Analysis of multi-hazards is highly relevant given anthropogenic climate change. Events such as floods and droughts already have significant socio-economic impacts (Barredo, 2007; Jonkman, 2005; Naumann et al., 2015; Zhang et al., 2011), and are expected to become more frequent and/or severe in the future (Dai, 2012, 2011a; Hirabayashi et al., 2013; Milly et al., 2002), although there exist uncertainties about whether these projected increases are caused by anthropogenic forcing. Numerous studies have investigated the combination of flood and drought events or, more generally, wet and dry hydrological extremes at local and regional scales, for both present and future climates (e.g. Berton et al., 2017; Collet et al., 2018; Deangelis et al., 1984; Di Baldassarre et al., 2017; Gil-Guirado et al., 2016; Oni et al., 2016; Parry et al., 2013; Pechlivanidis et al., 2017; Quesada-Montano et al., 2018; Yan et al., 2013; Yoon et al., 2018). Examples include the analysis of abrupt drought-flood transitions in China (Yan et al., 2013) and in England and Wales (Parry et al., 2013), the dynamical interplay between society and hydrological extremes (Di Baldassarre et al., 2017) and indices assessing the longterm evolution of vulnerability and adaptation to floods and droughts (Gil-Guirado et al., 2016). Other studies consider wet-dry interactions from a statistical perspective (Collet et al., 2018), or have related 
these two hazards to large-scale modes of climate variability (Cai and Rensch, 2012; Lee et al., 2018; Nobre et al., 2017; Siegert et al., 2001; Ward et al., 2014b; Yoon et al., 2018).

Quantifying wet and dry (also extreme) hydrological events at both regional and global scales is a nontrivial task. Some of the best indicators include the Palmer Drought Severity Index (PDSI) (Dai et al., 2004; Palmer, 1965), the Standardized Precipitation Index (SPI) (McKee T.B., Doesken N.J., 1995; McKee et al., 1993) and the Standardized Precipitation Evapotranspiration Index (SPEI) (VicenteSerrano et al., 2010). For instance, the PDSI was used to evaluate the combined effect of the Pacific Decadal Oscillation (PDO) and El Niño Southern Oscillation (ENSO) on global wet and dry changes over land, showing that when these two modes are in phase (e.g. El Niño-warm PDO) wet and dry events are amplified (Wang et al., 2014). The PDSI and SPEI have also been used to quantify wet and dry trends over China (Rubel and Kottek, 2010), with a generally good agreement between the two (Chen et al., 2017). At the global scale, the SPI and SPEI were used to explore wet and dry links with ENSO, PDO and the North Atlantic Oscillation (NAO) (Sun et al., 2016). The study found that ENSO has the greatest spatial impact for wet and dry changes, followed by the PDO having an effect in the American continent and eastern Russia, and the NAO affecting Europe as well as north Africa. Lastly, the SPI was also used in a global multi-model ensemble study of future projections in pluvial and drought events (Martin, 2018). This revealed that more severe pluvial events are expected in already wet regions and the same applies for more severe drought conditions in dry areas.

In this chapter, a relatively broad definition of multi-hazard events was adopted, including both the temporal (yet spatially separate) co-occurrence of wet and dry hydrological extremes at the global scale, and the rapid succession of hydrological extremes of opposite sign at the same location. It is worth emphasising that the term "hydrological extreme" does not necessarily imply observed flooding or drought events, unless explicitly mentioned, but simply hydrological observations exceeding a given threshold (hence extremes). The relevance of both types of hazards is evident. Stakeholders with geographically diverse portfolios, such as international corporations and (re)insurance companies, need to have a robust understanding of the co-occurrence statistics of natural hazards, because for example their supply chains can be obstructed by such events. They also need to account for the occurrence of damaging events in rapid succession, whose compound impacts may exceed the linear sum of the expected impacts for isolated wet and dry extremes. Similarly, estimates of the range of times that intervene between the two different extremes can inform emergency preparedness and prevention measures. Finally, national economies that are sensitive to agricultural output can be 
impacted by the occurrence of sequential hydrological extremes (Zampieri et al., 2017; Zhang et al., 2015).

Notwithstanding their socio-economic relevance, concurrent wet and dry hydrological extreme events at the global scale have seldom been addressed in the literature. One early study did consider combinations of wet and dry extremes via observed PDSI for two thresholds (wet, PDSI > 3 and dry, PDSI < -3) (Dai et al., 2004). This showed that the total global land area $\left(60^{\circ} \mathrm{S}-75^{\circ} \mathrm{N}\right)$ impacted by wet-dry extremes increased between 1950 and 2002, with marked changes occurring from the early 1970s and surface warming being attributed as the cause of these changes after the mid-1980s. This analysis has been extended in this chapter by: i) using an updated time series (1950-2014); ii) introducing new metrics for assessing concurrent wet-dry extremes; iii) presenting findings at monthly rather than annual resolution; and above all iv) defining the most geographically-widespread, but not necessarily contiguous, multi-hazard events, occurring within each month, instead of simply considering extreme observations with PDSI > 3 and PDSI < -3 . These multi-hazard properties have been explored using the monthly self-calibrated PDSI dataset (Dai, 2017; Sheffield et al., 2012). Specifically, the following questions are addressed:

1) How observed globally independent and concurrent wet-dry hydrological extreme events changed in the past?

2) What were the most spatially extensive independent and concurrent wet-dry hydrological extreme events?

3) How new metrics can help in better investigate concurrent wet-dry extremes?

4) How are these extremes related to different modes of climate variability?

In Section 5.2 the datasets, algorithm for computing the extreme events, new metrics and correlation with climate indices are described, along with their relevant statistical tests. Results are presented in Section 5.3. Thus, the land area impacted by the extreme events is shown in Section 5.3.1 (research question 1) and the most geographically-widespread events in Section 5.3.2 (research question 2). Results obtained from the new metrics and correlations with climate indices can be found in Sections 5.3.3-5.3.5, respectively answering research questions 3-4. Lastly, Section 5.4 provides the general discussion and conclusions, before a summary of the chapter is provided in Section 5.5. 


\subsection{Data and Methods}

\subsubsection{Data}

The self-calibrated monthly-mean Palmer Drought Severity Index based on the Penman-Monteith model (sc_PDSI_pm) (Dai, 2017; Sheffield et al., 2012) for the $1950-2014$ period, at $2.5^{\circ}$ horizontal resolution, was used. Self-calibration enables a more consistent comparison between different climatic regions, and the Penman-Monteith model outperforms the original PDSI Thornthwaite algorithm (Wells et al., 2004) in representing potential evaporation at the global scale (Sheffield et al., 2012). From this dataset, extreme wet and dry monthly observed events have been obtained, by filtering the data respectively for sc_PDSI_pm $\geq 3$ and sc_PDSI_pm $\leq-3$. These two thresholds represent very moist spells and severe droughts. Only grid-cells with time series having $\geq 95 \%$ of observations over the period of interest are considered.

Three climate modes of variability known to affect regional and global precipitation patterns have been further analysed: the Niño3.4 (Rayner et al., 2003; Trenberth, 1997), PDO (Mantua and Hare, 2002) and Atlantic Multidecadal Oscillation (AMO) (Schlesinger and Ramankutty, 1994). All these climate indices are at monthly time-resolution from 1950 to 2014, as issued by the National Oceanic \& Atmospheric Administration (NOAA). These climate indices have been chosen because they are the ones with largest geographical impacts, also in very-highly populated areas, i.e. North America and Europe. Other indices, such as the NAO (Barnston and Livezey, 1987), Pacific-North American (PNA) pattern (Barnston and Livezey, 1987) and Quasi-Biennial Oscillation (QBO) (Baldwin et al., 2001) have been also tested.

\subsubsection{Methods for identifying extreme wet, dry, neutral and wet-dry events}

First, the percentage of total land area impacted by the most widespread monthly extreme wet (sc_PDSI_pm $\geq 3$ ) and dry (sc_PDSI_pm $\leq$-3) hydrological events along with neutral $(-3<$ sc_PDSI_pm < 3) and extreme wet plus extreme dry events within the period 1950-2014 have been calculated. Monthly extreme wet events were derived following De Luca et al. (2017) by: (i) computing the wet annual maxima (AMAX), i.e. the highest sc_PDSI_pm observations within each calendar year, at each grid-cell, (ii) counting the number of wet AMAX observations by date, and (iii) taking the extreme wet event with the most geographically-widespread impacts, i.e. largest impacted area $(\mathrm{km} 2)$, within each month during 1950-2014. 
Extreme dry events were calculated in a similar way to extreme wet events except that in place of AMAX the sc_PDSI_pm annual minima (AMIN), i.e. the lowest sc_PDSI_pm observations within each year, were used to compute the extreme events. Neutral events were identified as follows: i) extract the sc_PDSI_pm AMAX of (non-extreme) wet events $\left(0 \leq \mathrm{sc} \_\right.$PDSI_pm $\left.<3\right)$; ii) extract the sc_PDSI_pm AMIN for (non-extreme) dry events $(-3<$ sc_PDSI_pm < 0); iii) pool within the same dataset both (non-extreme) wet/dry AMAX/AMIN events by month; iv) compute the most widespread neutral events by month as per above. Lastly, concurrent extreme wet-dry events were calculated by summing their relative impacted areas (\%) within each month. A Mann-Kendall test (Kendall, 1975; Mann, 1945) was performed to assess any significant trends within each time series. Relative Sen's slopes (Sen, 1968) with p-values were also computed.

Second, to establish whether the most widespread extreme wet, dry and wet-dry events were solely due to chance, a boot-strapping analysis of $n=10,000$ samples was performed from the original sc_PDSI_pm dataset. The boot-strapping steps were as follows: i) prepare the complete global (i.e. all grid-cells) sc_PDSI_pm dataset from 1950 to 2014; ii) sample, with replacement, the sc_PDSI_pm values $n=10,000$ times from this global dataset; iii) calculate $n=10,000$ extreme wet and $n=10,000$ extreme dry events with the same algorithm used above with the original dataset; iv) take the impacted area of the most geographically-widespread wet and dry events for each sample; v) calculate the mean and corresponding 95\% confidence intervals (c.i.) for the extreme wet and extreme dry events. Statistical significance was assessed by checking whether observed extreme wet or dry $\%$ of total impacted areas fell outside the $95 \%$ c.i. of the boot-strapped means. If this was the case, the observations were considered statistically significant. This boot-strapping procedure assumes spatial independence between each grid-cell and therefore it provides, although with replacement, a purely random output, which is what one can expect from a boot-strapping analysis.

\subsubsection{Wet-dry metrics}

The wet-dry (WD) ratio is derived on a cell by cell basis by taking the natural logarithm of the total number of extreme wet observations (months with sc_PDSI_pm $\geq 3$ ) divided by the total number of extreme dry observations (months with sc_PDSI_pm $\leq-3$ ) over the 1950-2014 period:

$$
W D-\operatorname{ratio}_{h}=\ln \left(\sum_{i=1}^{n_{i}} W_{e t} / \sum_{j=1}^{n_{j}} \operatorname{Dry}_{j, h}\right)
$$


where $h$ refers to a single grid-cell and $n_{i}$ and $n_{j}$ are the total number of wet and dry events, respectively. The WD-ratio gives information about the propensity of a given area to be more affected by wet or dry extremes. Thus, WD-ratio $>0$ signifies that wet extremes outnumber dry extremes and WD-ratio $<0$ indicates a predominance of dry extremes over wet ones. The natural logarithm was used to narrow the range of WD-ratio values and to separate the wet-dominated versus dry-dominated regions by sign. As a caveat, it is worth noting that the WD ratio does not account for the different intensities of wet and dry extremes.

Wet to dry and dry to wet transitions, named extreme transitions (ET) were assessed for each grid-cell by computing the average time interval (months) between these events, within the 1950-2014 period. More specifically, ET for wet to dry was derived as follows, for each grid-cell: i) extract both wet (sc_PDSI_pm $\geq 3$ ) and dry (sc_PDSI_pm $\leq-3)$ extreme observations from the entire (1950-2014) sc_PDSI_pm dataset; ii) order observations by time, from oldest to the most recent; iii) retain only the earliest event in the case of consecutive extreme dry observations and the latest in the case of consecutive wet observations; iv) calculate the time interval (monthly difference) between wet and dry observations within the time-series; and v) take the average of the time interval. The same algorithm was applied for calculating ET from dry to wet for each grid-cell, with the only difference being in step iii) where the earliest wet and latest dry observations are kept and in step iv) where the time interval is now calculated between dry to wet transitions.

To check the statistical significance of the observed ET, a boot-strapping analysis with $\mathrm{n}=1,000$ samples (10,000 samples were not possible due to high computational demand) was performed in a similar way as for the most widespread extreme wet, dry and wet-dry events. In this analysis, for each grid-cell, the following steps were followed: i) from the main sc_PDSI_pm dataset extract and flag extreme wet (sc_PDSI_pm $\geq 3$ ) and extreme dry (sc_PDSI_pm $\leq-3$ ) observations; ii) sample, with replacement, $n=1,000$ of these wet and dry observations with the corresponding (sampled) date (yearmonth); iii) calculated $n=1,000$ ET from wet to dry and $n=1,000$ ET from dry to wet using the same algorithm as per above; and iv) take the ET (wet to dry and dry to wet) means with the relative $95 \%$ c.i. of the means. Statistical significance was assessed by checking, for each grid-cell, if the observed ET time interval (wet to dry and dry to wet) mean fell outside the $95 \%$ c.i. of the boot-strapped ET mean. If this was the case the observation was considered statistically significant. Also here, the bootstrapping assumes spatial independence between grid-cells. 


\subsubsection{Correlation tests}

Associations between extreme wet-dry hydrological extremes and the three modes of climate variability (Niño3.4, PDO and AMO) were assessed using Spearman's rank correlation test. Specifically, the correlations were performed for each grid-cell using monthly wet and dry extreme observations (sc_PDSI_pm $\geq 3$ and sc_PDSI_pm $\leq-3$ ) paired with the corresponding monthly values of Niño3.4, PDO and AMO. Spearman's test does not require data to be normally distributed, which is the case for the wet-dry extreme PDSI values. The wet-dry extreme datasets were computed, for each grid-cell, by adding together the extreme wet and dry monthly observations within the period 1950-2014. Since the number of correlation tests performed is large $(>2,700)$ there is a risk of incurring in statistically significant results simply by chance. Thus, to account for Type I errors (or 'false positives') the Bonferroni correction (Bonferroni, 1936; Sedgwick, 2014) was applied to all pvalues.

Finally, since Niño3.4 may affect correlations with other modes of climate variability, this signal was removed from the PDO and AMO by performing partial correlations, with the R programming package 'ppcor' (see documentation here: https://cran.r-project.org/web/packages/ppcor/ppcor.pdf) (Kim, 2015). Partial correlations may be regarded as the association between two variables after removing the effect of one or more other variables. In this analysis, the partial correlations, between two variables $x_{i}$ (e.g. PDO) and $x_{j}$ (e.g. sc_PDSI_pm) given a third variable $x_{k}$ (e.g. Niño3.4) is defined as follows (Kim, 2015; Whittaker, 2009):

$$
r_{i j \mid k}=\frac{r_{i j}-r_{i k} r_{j k}}{\sqrt{1-r_{i k}^{2}} \sqrt{1-r_{j k}^{2}}}
$$

In this study the partial correlations have been computed, using the Spearman's correlation coefficient, for PDO and AMO, given the Niño3.4, with respect to sc_PDSI_pm extreme wet and dry observations.

\subsection{Results}

\subsubsection{Land area impacted by extreme wet, dry, neutral and wet-dry events}

The percentage $(\%)$ of total global land area impacted by the most widespread extreme wet, dry and neutral events is shown in Figure 5.1, at monthly resolution from 1950 to 2014. For extreme wet events (Figure 5.1a, sc_PDSI_pm $\geq 3$ ) the average impacted area over the 65 -year period is $2.17 \%$. The most 
widespread wet event occurred in December 2010 (7.77\%, discussed in Section 5.3.2). The MannKendall test indicates a positive, though non-significant trend (with Sen's slope $=1.6 \mathrm{e}-04$ ). The nonsignificant observed growth in extreme wet area is indeed not consistent with previous works (Dai, 2011b; Dai et al., 2004).

For extreme dry events (Figure 5.1b, sc_PDSI_pm $\leq-3$ ) the average area is $2.38 \%$ and the largest event occurred in January 2003 (8.57\%, discussed in section 5.3.2). In this case, the Mann-Kendall test indicates a positive and statistically significant ( $\mathrm{p}$-value $<<0.01$ ) trend (with Sen's slope $=1.7 \mathrm{e}$ 03). This signifies that the total area subject to severe drought increased between 1950 and 2014. This result agrees with previous studies showing a global increase in drought risk, attributed to anthropogenic climate change, in both observations and climate model simulations (Dai, 2012, 2011a, 2011b; Dai et al., 2004; Marvel et al., 2019). Such changes in drought are linked to anomalies in tropical sea surface temperatures (SSTs) and therefore driven by both El Niño and La Niña phases, along with increased surface warming since the 1980s.

The most widespread neutral events (Figure 5.1c, $-3<$ sc_PDSI_pm < 3) affect on average 13.64\% of the global land area over the 1950-2014 period. The Mann-Kendall test shows a negative and significant ( $\mathrm{p}$-value $<<0.01$ ) trend (with Sen's slope $=-1.9 \mathrm{e}-03$ ). Such a reduction in the area under neutral conditions is explained by the observed increasing trend of both extreme wet and dry events. The neutral events show strong seasonality, with peaks of impacted area occurring during December. This resembles what is seen for the most widespread wet and dry events, which also tend to occur mostly during boreal winter. However, the fact that $73.4 \%$ of the global sc_PDSI_pm land area is in the northern hemisphere is no doubt a factor.

Finally, the area with concurrent wet-dry hydrological extremes (Figure 5.1d) shows an increasing $($ Sen's slope $=1.08 \mathrm{e}-03)$ and statistically significant $(\mathrm{p}$-value $<<0.01)$ trend, again in agreement with shorter records (Dai, 2011b; Dai et al., 2004). The mean monthly value of total global area with concurrent wet-dry extreme events is $4.56 \%$. 

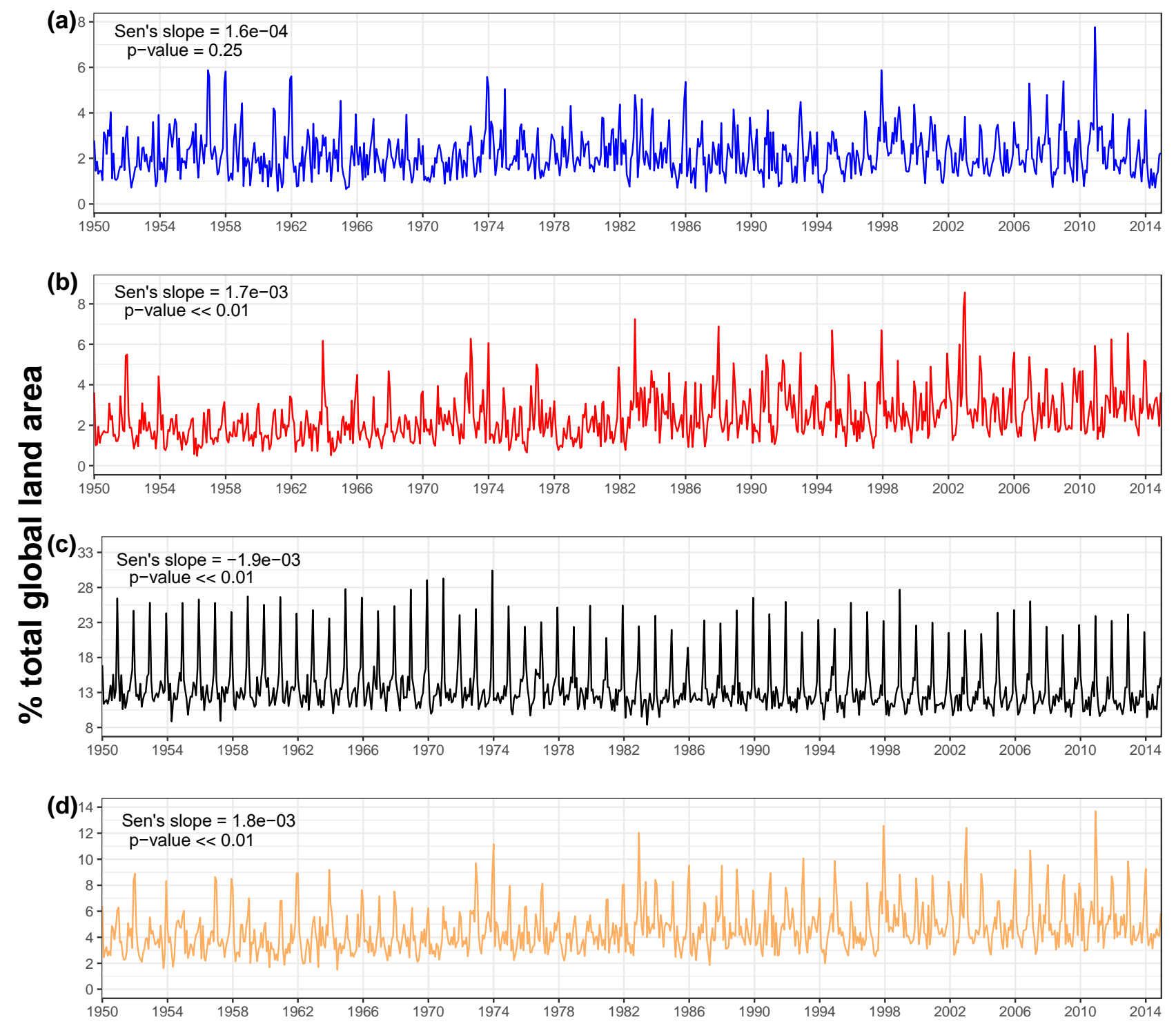

\section{Time (months)}

$\square$ wet $\square$ dry $\square$ neu $\square$ wet-dry

Figure 5.1 Percentage (\%) of total land area with (a) wet (blue), (b) dry (red) extremes, (c) neutral (black) and (d) extreme wet + extreme dry (orange) events over the 1950-2014 period. Wet extremes are sc_PDSI_pm $\geq 3$ ) and dry extremes sc_PDSI_pm $\leq-3$ monthly observations. Sen's slopes and the significance of the Mann Kendall test (p-values) are shown in each panel. 


\subsubsection{Concurrent global flood and drought events}

Next, the single most extensive wet, wet-dry and dry events have been considered, and it is shown that they capture recorded severe drought and flood events around the world. The most widespread global extreme wet event was also the most widespread wet-dry event, and occurred in December 2010 (Figure 5.2a). Recorded events matching this occurrence include the devastating Queensland floods in Australia (BBC, 2010a; Smith et al., 2013; Trenberth and Fasullo, 2012; Zhong et al., 2013); heavy floods and landslides occurring in south-east India killing more than 180 people (Reliefweb, 2010); widespread flooding and landslides over Colombia and Venezuela causing about 300 deaths and leaving thousands homeless (BBC, 2010b; Telegraph, 2010; Trenberth and Fasullo, 2012); and flooding affecting the north-western USA (NWRFC, 2010). Severe wet conditions were also recorded in central-eastern Europe, however, no significant damages were reported by the media. Such a widespread wet event impacted $7.77 \%$ of the total global land area. December 2010 was characterized by a very strong negative Niño3.4 phase, within the 2010-2012 La Niña event (Luo et al., 2017). Moreover, the PDO and AMO were respectively in their cold and positive phases. The same phases occurred during November 2010 (not shown), and these antecedent conditions may have contributed to the extreme wet and dry events in the sc_PDSI_pm series (Lee et al., 2018). At the same time, droughts were recorded in central Asia, Madagascar, the Horn of Africa (BBC, 2011), south America, eastern USA (NOAA, 2011) and north Canada, covering a total of 5.93\% of land area. Both the extreme wet and dry percentages $(\%)$ of land area impacted are significantly different from their respective values expected by chance, i.e. $6.96 \%$ and $7.61 \%$ (95\% c.i.).

The most widespread extreme dry event occurred during January 2003 with $8.57 \%$ of total land area impacted by drought and 3.84\% of land experiencing wet hydrological extremes and floods (Figure 5.2b). During this event, eastern Australia was the most affected region, experiencing the worst drought in 20 years, which was driven by an El Niño event that lead to severe dust storms and bushfires (Gabric et al., 2010; Horridge et al., 2005; Levinson and Waple, 2004; McAlpine et al., 2007). This episode belongs to the so called 'Millennium Drought' (Van Dijk et al., 2013) which affected Australia between 2001 and 2009. Other regions experiencing severe drought during January 2003 were northeast China, India (Sinha et al., 2016), Scandinavia (Irannezhad et al., 2017), west Africa, parts of Brazil and a few scattered areas between Mexico, USA, Canada, Russia and Indonesia. January 2003 was an El Niño month with the Niño3.4 index being in a positive phase at the same time as a warm PDO phase. On the other hand, the AMO registered an almost neutral phase. As for the December 2010 episode in Figure 5.2a, such climate patterns occurred also in the previous month. Meanwhile, other regions experienced wet hydrological extremes and floods, such as south-east China, central Russia, 
Europe, southern Great Britain (BBC, 2003; Marsh, 2004), Madagascar (Reliefweb, 2003), Argentina, Chile and scattered parts of Africa and Canada (DFO, 2008). The \% of land area impacted by both extreme wet and dry events during January 2003 was significantly different from the value expected by chance (see above, $95 \%$ c.i.). 

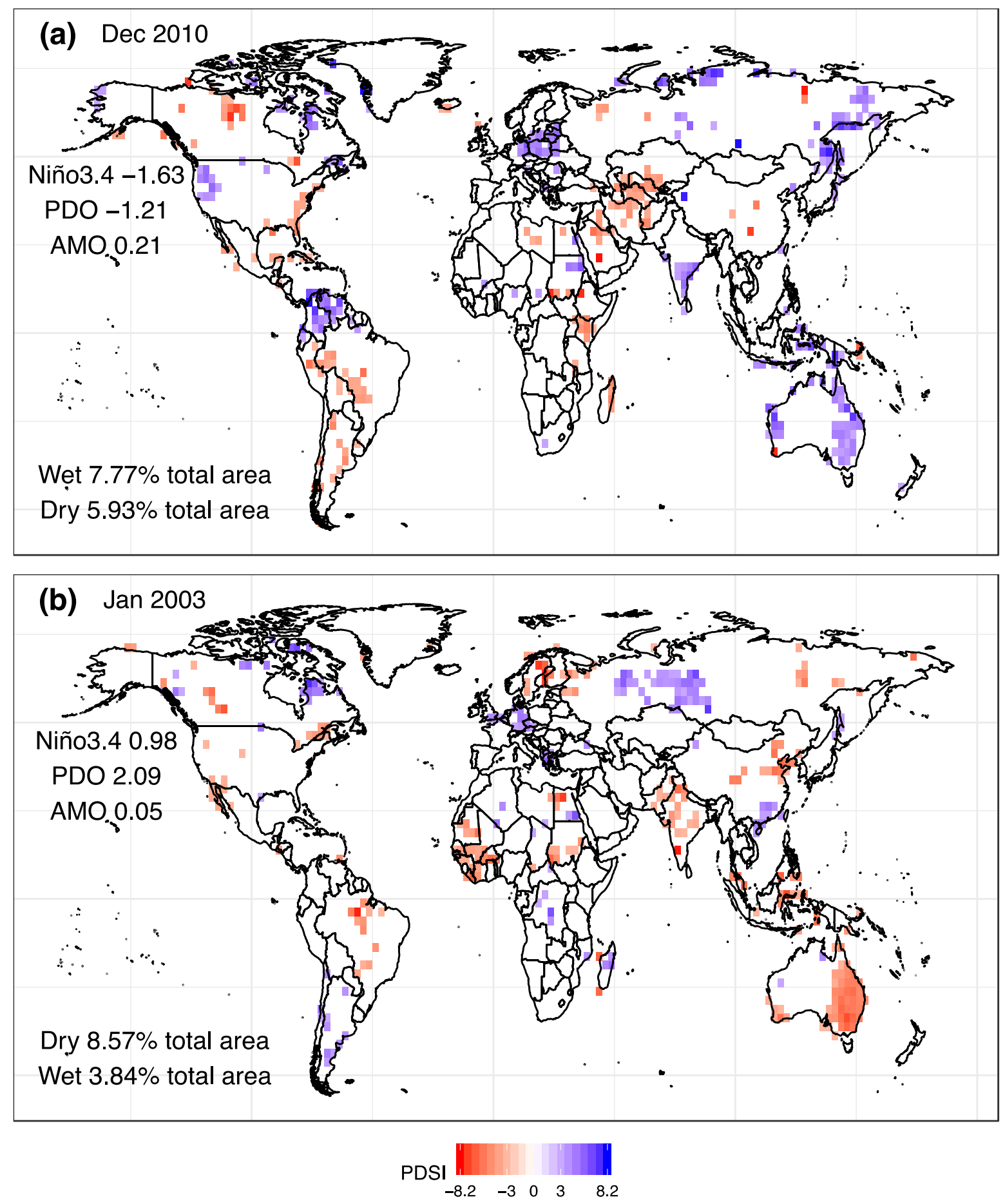

Figure 5.2 (a) Most widespread extreme global wet hydrological event (blue colour) and coincident extreme dry areas (red colour), December 2010. The event was also the most widespread concurrent wet-dry episode. The percentage (\%) of total land area is shown for both wet and dry extremes, along with the values of the three climate indices (i.e. Niño3.4, PDO and AMO) in December 2010. (b) As (a) but for the most widespread extreme global dry hydrological event, January 2003. (a)-(b) The analysis is based on the self-calibrated monthly mean Palmer Drought Severity Index (sc_PDSI_pm) for the period 1950-2014. 


\subsubsection{Wet-dry (WD) ratio}

The WD-ratio highlights regions that experienced higher or lower frequencies of wet or dry hydrological extremes (Figure 5.3). The patterns identified in this analysis represent the 65-year propensity for extreme wet or extreme dry events in a given area. Hot spots for extreme wet tendency emerge in the USA, northern Mexico, Colombia, Venezuela, Argentina, Bolivia, Paraguay, northern Europe, North Africa, eastern China and Australia. On the other hand, regions with higher frequencies of extreme dry events are found in Canada, central south America, central and southern Europe/Africa, western China and south-western Australia. Other regions, such as Russia, display mixed patterns. These WD-ratio patterns are in agreement with global trends in drought over the 1950-2010 period, identified using the sc_PDSI_pm dataset (Dai, 2012).

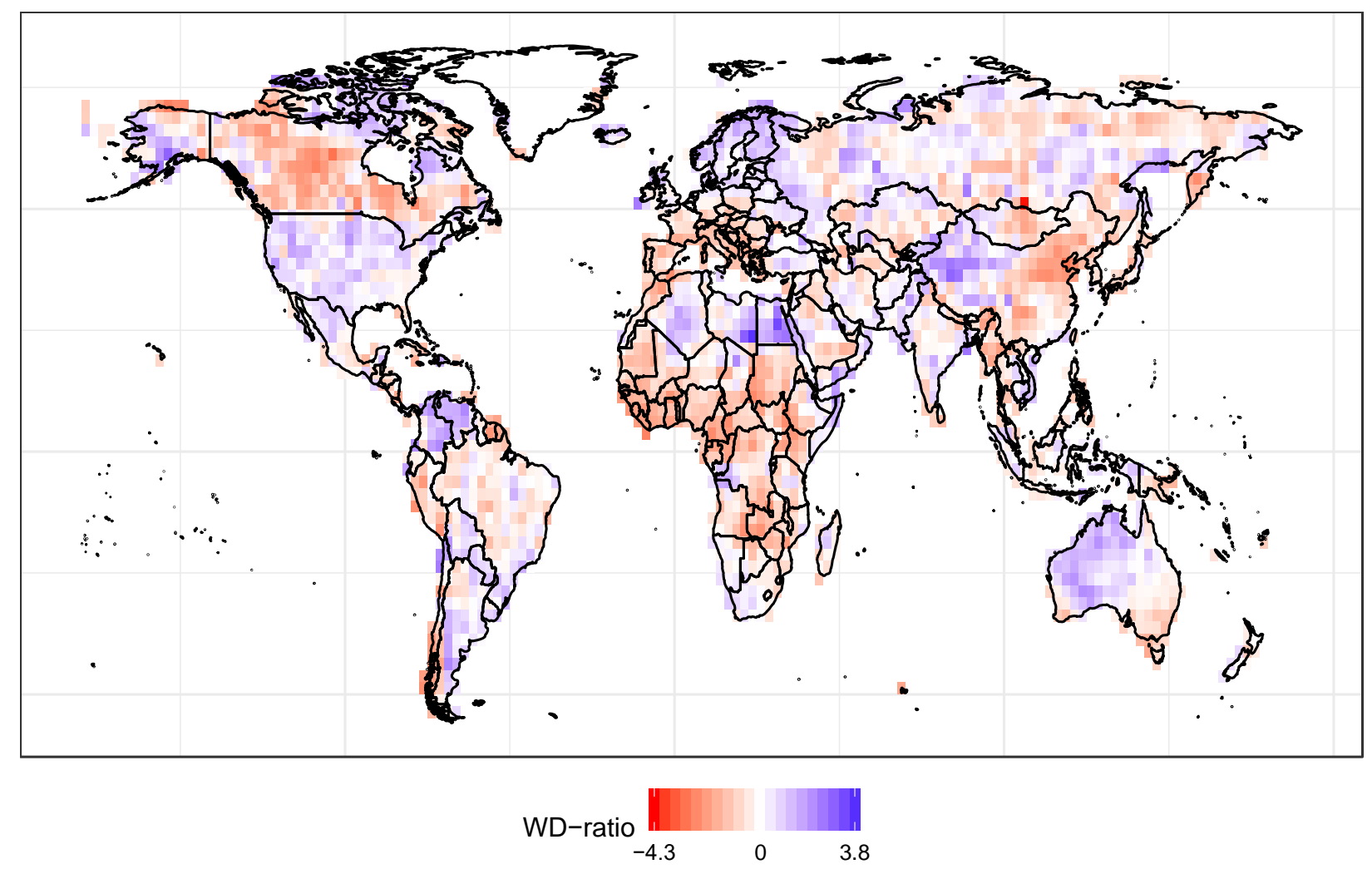

Figure 5.3 Wet-dry (WD) ratio derived for every grid-cell. Blue colours (WD-ratio >0) mean that the area experienced more wet than dry hydrological extremes. Red colours $(W D$-ratio $<0)$ indicate the opposite. 


\subsubsection{Extreme transitions (ET)}

Figure 5.4a shows the extreme transitions (ET), in time interval (months), from wet to dry and dry to wet extreme events within the period 1950-2014 plotted against the percentage of total land area impacted. The ET from wet to dry (blue curve) exhibits a peak at 22-months with $4.3 \%$ of total land having this mean time interval. On the other hand, ET from dry to wet (red curve) peaks at 18-month with almost $5 \%$ of global land having this average separation time. Overall, ET from wet to dry takes longer than ET from dry to wet. According to the boot-strap analysis, the ET for wet to dry extremes are statistically significant in all grid-cells; for dry to wet only 10 grid-cells are not different from chance. The cumulative distribution functions (CDFs) of wet to dry and dry to wet ET time intervals are also shown (Figure 5.4b). For wet to dry 50\% of the ET occur within $\sim 27$ months, whereas for dry to wet $50 \%$ of ET are observed within 21 months. The two ET distributions are significantly different (p-value $<<0.01$, Mann-Whitney-Wilcoxon test) under the null hypothesis that their means are equal. 

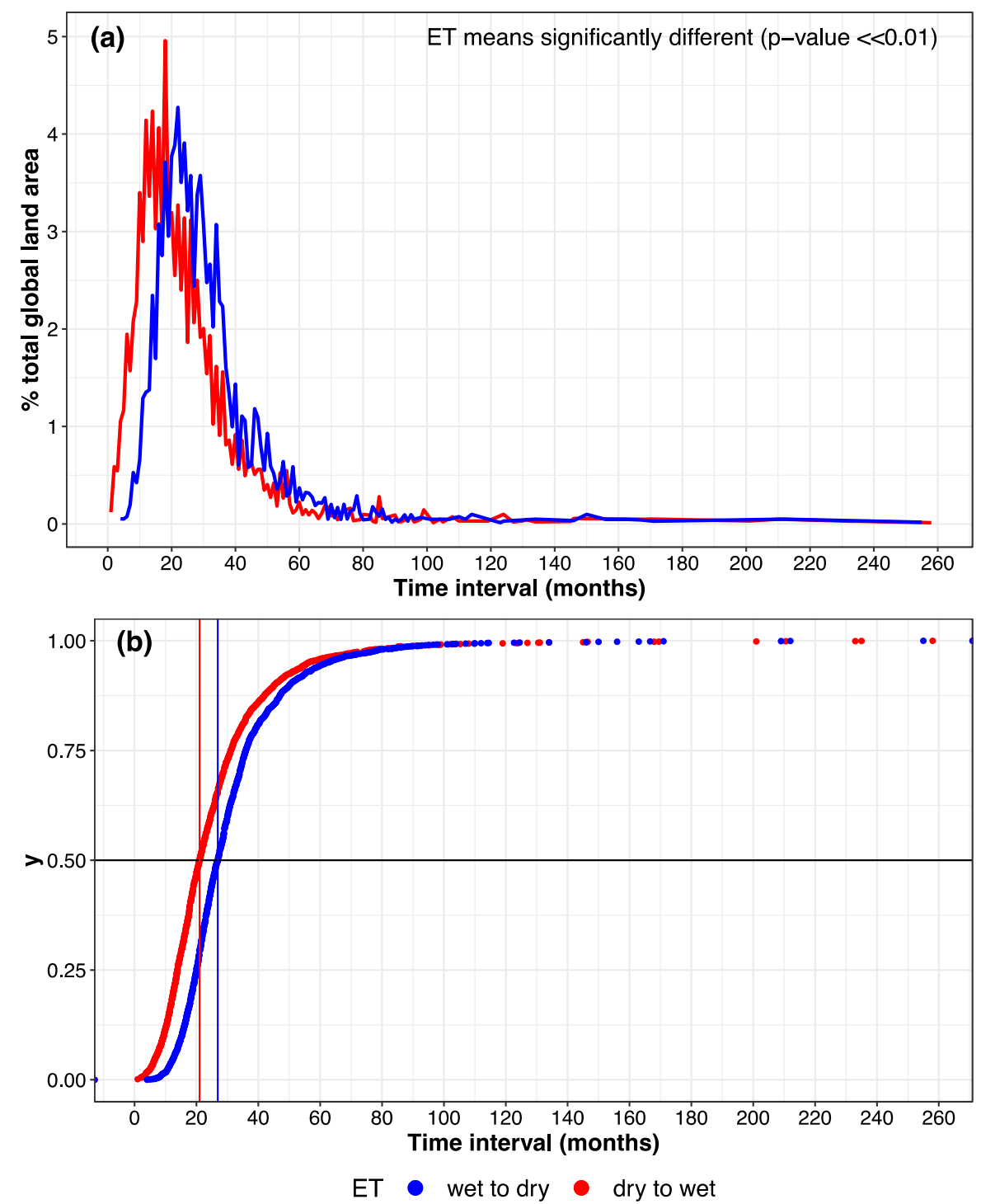

Figure 5.4 Extreme transition (ET) time intervals between extreme wet to dry (blue) and between extreme dry to wet (red). (a) Total percentage (\%) of total land area impacted as a function of ET and (b) cumulative distribution functions (CDFs). The horizontal black line in (b) indicates the 50th quantile (i.e. median) of the distribution and the blue and red lines the respective ET time intervals. The two distributions show a statistically significant difference in their means (p-value $<<0.01$, Mann-Whitney-Wilcoxon test). 
Moreover, ET from dry to dry and wet to wet were also computed (Figure 5.5). Dry to dry time intervals peak at 27 months with $3.21 \%$ of land area impacted, whereas wet to wet time intervals peak at 30 months with $3.14 \%$ of area affected (Figure 5.5a). For dry to dry ET 50\% occurred within $\sim 37$ months and the same but for wet to wet ET in $~ 38$ months (Figure 5.5b). A Mann-Whitney-Wilcoxon test shows that the two distributions are significantly different ( $\mathrm{p}$-value $<0.01$ ), under the null hypothesis that their means are equal as per the multi-hazards case.
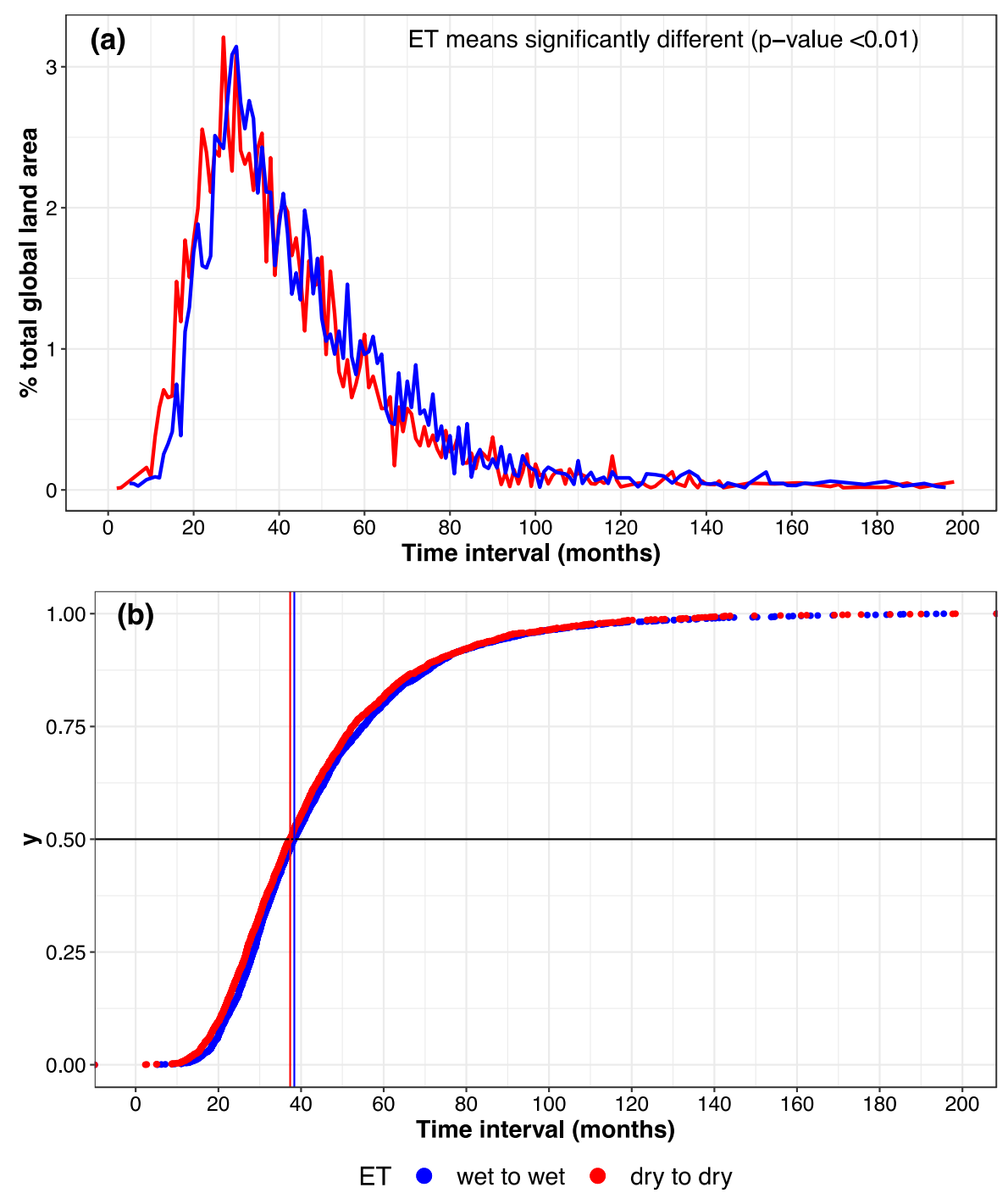

Figure 5.5 Extreme transition (ET) time intervals between extreme wet to wet (blue) and between extreme dry to dry (red). (a) Total percentage (\%) of total land area impacted as a function of ET and (b) cumulative distribution functions (CDFs). The horizontal black line in (b) indicates the 50th quantile (i.e. median) of the distribution and the blue and red lines the respective ET time intervals. The two distributions show a statistically significant difference in their means ( $p$-value $<<0.01$, Mann-Whitney-Wilcoxon test). To note that for simplicity only ET with a time interval $\leq 200$ months are shown. 


\subsubsection{Correlations with climate indices}

Figure 5.6 shows global correlations between hydrological extremes (wet and dry) and the three major modes of climate variability (Niño3.4, PDO and AMO), which are known to have a significant effect on regional precipitation/temperature patterns and hence flood and drought events. Details of the calculation are provided in Section 5.2.4. The same correlation tests have been also computed for the NAO (Barnston and Livezey, 1987), PNA (Barnston and Livezey, 1987) and QBO (Baldwin et al., 2001), however the correlation coefficients were weaker compared to Figure 5.6 and very few of them were statistically significant, as shown in Figure 5.7.

ENSO is one of the modes with the most widespread global impacts and in this study is represented by the most widely used configuration, i.e. the Niño3.4 index (Figure 5.6a). The positive phase of Niño3.4 (which can lead to El Niño events), is negatively correlated (p-value $<0.05$ ) with extreme wet sc_PDSI_pm values over parts of central Canada, northern South America, southern Africa, India, central China, central and northern Russia, Indonesia and eastern Australia. On the other hand, positive and significant correlations are found over southern USA, in some scattered regions of central and southern south-America and in the Middle East. This implies that a positive Niño3.4 phase is associated with a lower likelihood of extreme wet hydrological events (sc_PDSI_pm $\geq 3$ ) in these regions, whereas negative Niño3.4 phase (possibly leading to La Niña events) typically enhances such extremes.

In Figure 5.6b-c global partial correlations between hydrological extremes, measured with sc_PDSI_pm, and PDO/AMO modes of climate variability with the ENSO signal removed are shown. Correlations for PDO (Figure 5.6b) generally resemble the spatial patterns found for Niño3.4. In this study, negative correlations are also found in north-western North-America, equatorial Africa and eastern Russia. At the same time, almost all significant correlations over Australia, China and India vanish. On the other hand, positive correlations are found in central-western USA, southern SouthAmerica and Kazakhstan. The fact that Niño3.4 and PDO correlations show very similar spatial patterns (Figures 5.6a-b) suggests that when these two indices are in phase (i.e. El Niño-warm PDO and La Niña-cold PDO), wet and dry changes are magnified (Wang et al., 2014). The correlation pattern shown in Figure 5.6b also agrees with season-ahead peak river flow correlations with the PDO (Lee et al., 2018).

The pattern of AMO correlations (Figure 5.6c) differs from Niño3.4 and PDO indices and returns a greater number of significant (p-value <0.05) grid-boxes than Niño3.4 (2.5\% more grid-cells overall). 
For the AMO, negative and significant correlations are found in Brazil, Argentina, Mexico, scattered areas in north America, the Horn of Africa and eastern China. Positive correlations are found in the Sahel region of Africa, Russia and central Asia. These results are again in agreement with global season-ahead correlations found between peak river flows and AMO (Lee et al., 2018). 

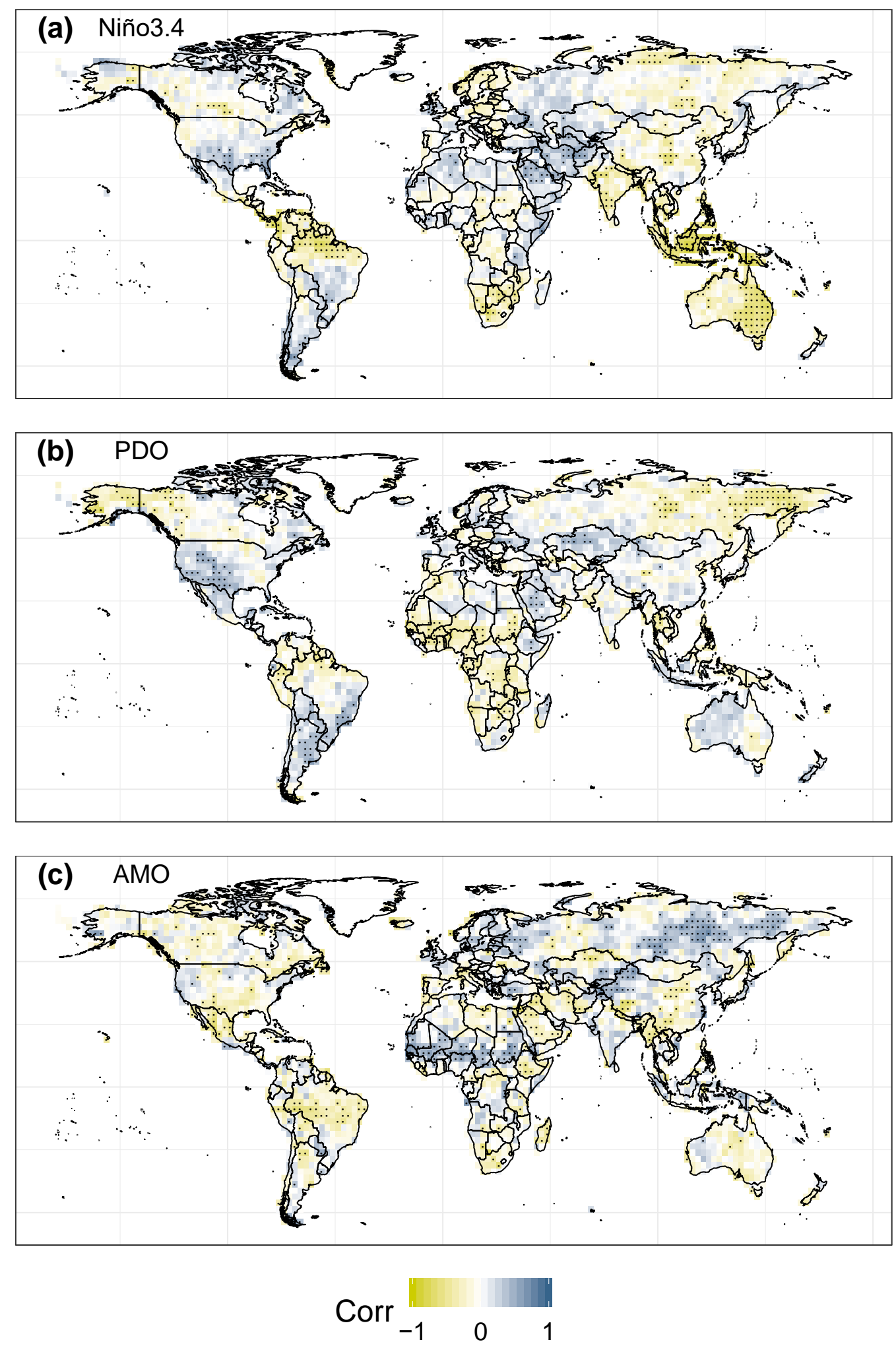

Figure 5.6 Correlations between extreme wet (sc_PDSI_pm $\geq 3$ ) and dry (sc_PDSI_pm $\leq-3)$ hydrological events and (a) Niño3.4, (b) PDO and (c) AMO. For (b) and (c) partial correlations are performed to remove the Niño3.4 signal. Correlations and partial correlations make use of the Spearman's correlation coefficient. Correlations significant at the $5 \%$ level (p-value <0.05) are shown by stippling. The Bonferroni correction was applied to all p-values. In (b) and (c) the ENSO signal has been removed via partial correlations. 

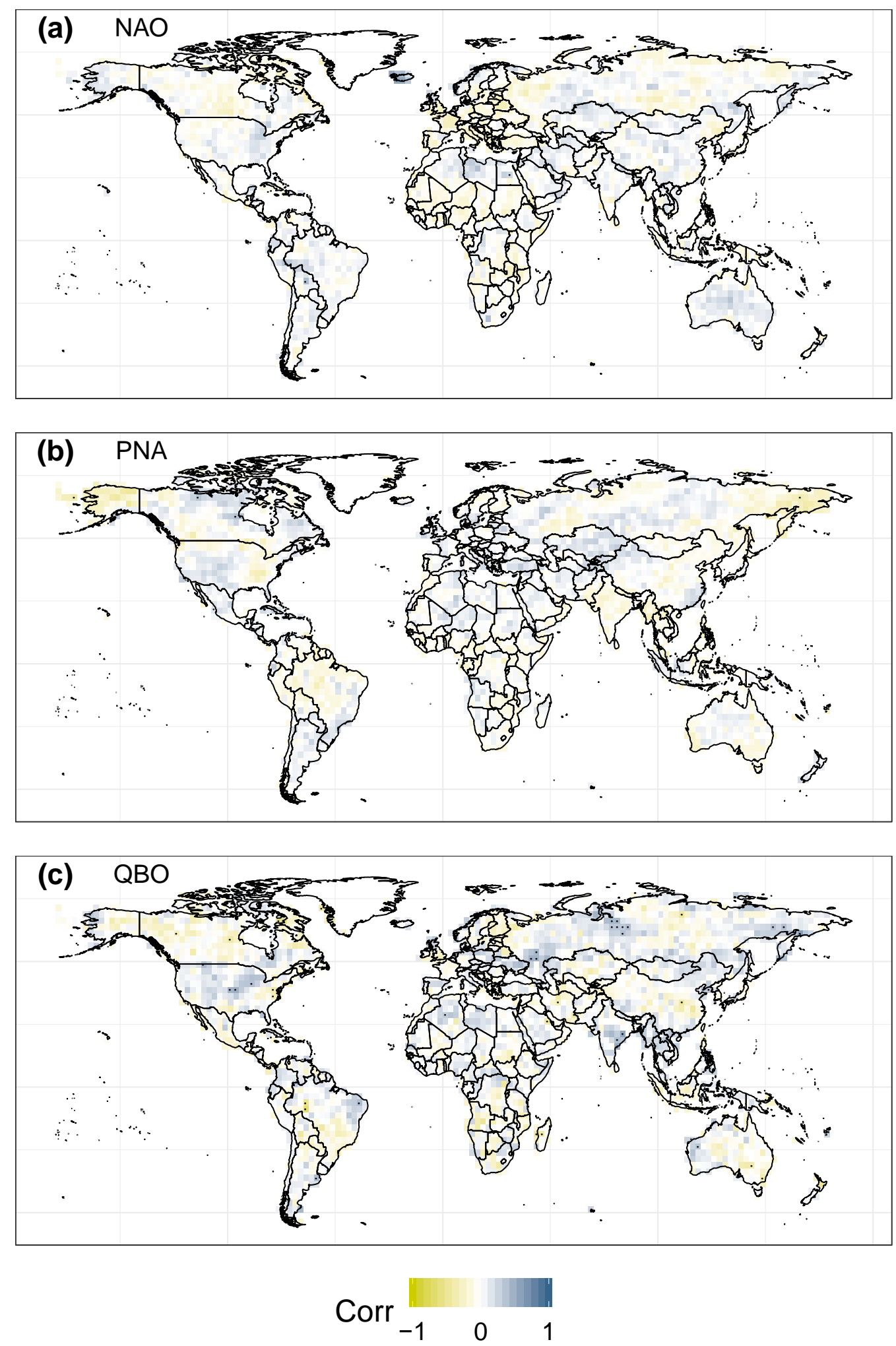

Figure 5.7 Correlations between extreme wet (sc_PDSI_pm $\geq 3$ ) and dry (sc_PDSI_pm $\leq-3)$ hydrological events and (a) NAO, (b) PNA and (c) QBO. Correlations and partial correlations make use of the Spearman's correlation coefficient. Correlations significant at the 5\% level (p-value <0.05) are shown by stippling. The Bonferroni correction was applied to all p-values. 


\subsection{Discussion and Conclusions}

Natural hazards, such as wet and dry extremes, can coincide over time and space creating multi-hazard events in turn leading to significant socio-economic losses. Geographically remote co-occurring extremes pose a potential economic risk to stakeholders with global assets and/or supply chains. For instance, knowledge of recurrent patterns of coincident hydrological extremes could be used to hedge losses, in regional hydropower production ( $\mathrm{Ng}$ et al., 2017; Turner et al., 2017) and/or with respect to crop yield (Leng and Hall, 2019; Xie et al., 2018; Zampieri et al., 2017), planting and harvesting (Sacks et al., 2010). Relatively rapid successions of extremes at the same location pose challenges for disaster preparedness, management and risk reduction. Thus, the maps shown in Figures 5.2 can therefore be valuable for example for quantifying future concurrent wet-dry extreme events, within a global seasonal forecasting product. Floods and droughts are expected to become more frequent and severe in the future due to anthropogenic climate change (Arnell and Gosling, 2016; Dai, 2012; Hirabayashi et al., 2013; IPCC, 2012), underscoring the importance of research on multi-hazard hydrological extremes.

This chapter investigated a range of multi-hazard hydrological extremes at the global scale during the period 1950 to 2014, with the primary objective of identifying the most geographically-widespread events. The secondary goal was to develop new metrics for describing some of the properties of wetdry extremes, namely combinations of wet and dry extremes at locations, or their succession at a given location. Answer to research question 1 is that the land area affected by extreme dry and geographically remote wet-dry events has increasing and statistically significant trends. This coincides with the expectation that such hazards are set to increase due to anthropogenic climate change (e.g. Güneralp et al., 2015; Hirabayashi et al., 2013; IPCC, 2012), and is in agreement with previous studies (Dai, 2012, 2011b; Dai et al., 2004). In this chapter, however, a more stringent definition of extreme events (De Luca et al., 2017), which captures well-known flooding and drought episodes has been applied. It is further shown that these extremes can have global-scale impacts, by giving emphasis to the most widespread wet, dry and wet-dry events. It is also found that the most widespread extreme wet and wet-dry events occurred during December 2010 and the most extreme dry events during January 2003. Such events provide an answer to research question 2 and they also coincided with well documented floods and droughts occurring in remote regions across the globe.

Furthermore, research question 3 has been addressed by introducing two new metrics, the wet-dry (WD) ratio and the extreme transition (ET) between wet/dry and dry/wet extremes. The former 
discerns the local predominance of extreme wet or extreme dry events, by considering both types of extremes simultaneously. Areas experiencing more wet than dry extremes were detected in the USA, northern and southern South-America, northern Europe and North Africa, western China and most of Australia. More dry than wet extremes were experienced in most of the remaining areas. Such knowledge, if transferred into future projections, could raise awareness amongst emergency planners, (re)insurance companies, farmers, relief organizations, and international agencies to better plan and adapt to extreme wet and dry hydrological events. The ET metric estimates for every grid-cell the average time interval between opposing extremes (i.e. transitions from wet to dry and from dry to wet). The results show that the two ET time-series' means differ significantly (p-value $<<0.01$ ) and that ET between wet to dry are on average slower than dry to wet (22-versus 18 -month interval on average). Knowing long-term changes in ET time intervals between wet to dry and dry to wet hydrological extremes can be significant for emergency planners, local businesses, governments and stakeholders. For example, if average intervals shorten, losses could accrue more frequently, causing recurrent shocks to local economies and, in some regions, impeding development.

The results obtained from the WD-ratio showed that some regions experienced more wet (dry) than dry (wet) extremes in the past. In this chapter, the physical mechanisms driving such spatial patterns were not explicitly investigated. However, since the sc_PDSI_pm dataset is computed by making use of temperature and precipitation data, both dynamical and thermodynamic changes in the large-scale atmospheric configuration (e.g. Harrington et al., 2019; Pfahl et al., 2017) can affect the spatiotemporal patterns of sc_PDSI_pm wet and dry extremes. This implies for example that storm tracks, blocking, localised convection, along with the increased water-holding capacity of the atmosphere due to warmer temperatures (Trenberth, 2011) may have played a role in shaping the observed predominance of wet and dry extremes. Similarly, mechanisms driving ET from wet (dry) to dry (wet) were not addressed in this chapter. However, wet to dry ET happen on a slightly longer time-scale than dry to wet ET, possibly implying that for the soil it is necessary more time to dry from a wet condition than to become wet from dry. Such tentative explanation is similar to what can be driving a flashflood, i.e. heavy rainfall falling into dry soil with poor absorption ability (e.g. Collier, 2007; Doswell et al., 1996).

The analysis in this chapter was based on the self-calibrated monthly-mean Palmer Drought Severity Index, as it has been previously used in similar global studies (e.g. Dai, 2012, 2011b; Dai et al., 2004). Future research opportunities include the use of other indices, such as the Standardized Precipitation Index (McKee T.B., Doesken N.J., 1995; McKee et al., 1993) or the Standardized Precipitation 
Evapotranspiration Index (Vicente-Serrano et al., 2010) to validate the findings and to account for uncertainty in the observations of concurrent wet-dry extremes. Emphasis should also be given to evaluating the seasonality of the extremes, since more robust and meaningful patterns could emerge, specifically with respect to correlations with modes of climate variability. Similar analyses could be applied to single Köppen climate zones (Rubel and Kottek, 2010) to discern possible regional variations in concurrent wet-dry extreme characteristics. Finally, once baseline maps and data for hydrological multi-hazards have been established from observations, the next step could be to investigate with climate model output how these phenomena may change under anthropogenic climate change.

To this end, and for providing an answer to research question 4, it is important to identify possible climate drivers of the observed hydrological extremes. In this study, correlations between wet-dry hydrological extremes and the corresponding values of the ENSO, PDO and AMO indices have been computed. The global correlation patterns for ENSO confirm previous studies (Emerton et al., 2017; Lee et al., 2018; Wang et al., 2014; Ward et al., 2014b), that investigated the effect of both El Niño and La Niña events on global flood hazard and global season-ahead correlations between river peak flows and climate indices, such as ENSO, PDO and AMO. It is worth highlighting that ENSO-induced wet and dry changes are magnified when in phase with the PDO index. Global correlations are found between the sc_PDSI_pm and PDO/AMO too. The PDO patterns generally match those of ENSO, and this confirms the finding that when ENSO and PDO are in phase they amplify the global wet and dry changes (Wang et al., 2014). The AMO, however, shows different and in some cases opposite correlation patterns when compared to ENSO and PDO. Correlations of hydrological extremes with modes of climate variability can be helpful for seasonal and sub-seasonal hydrological forecasting and, in this case, they provide information about what kind of climate index phase is driving wet and dry extremes in different regions globally. They also point to physical mechanisms, linked to climate dynamics, which may have not been uncovered yet.

\subsection{Summary}

The research conducted in this Chapter focussed on a global analysis of observed concurrent wet and dry hydrological extremes and their links with modes of climate variability (De Luca et al., 2019c). The research made use of the self-calibrated monthly PDSI dataset based on the Penman-Monteith model (Dai, 2017; Sheffield et al., 2012), along with the ENSO, PDO and AMO indices within the 
1950-2014 period. Results showed that the land area impacted by dry and concurrent wet-dry hydrological extremes increased significantly over the observational period. The most geographically widespread wet, dry and concurrent wet-dry events were also identified and they match with wellknown flooding and drought episodes. Two new metrics were also introduced, namely the Wet-Dry (WD) ratio and the extreme transitions (ET) between wet/dry and dry/wet extreme observations. The former identifies the local predominance of extreme wet or extreme dry events. For example, areas experiencing more wet than dry extremes were detected in the USA, northern and southern SouthAmerica, northern Europe and North Africa, western China and most of Australia. Whereas the ET estimates, for every grid-cell, the average time interval between opposing extremes. Results showed that the two ET time-series' (wet to dry and dry to wet) means differ significantly and that ET between wet to dry are on average slower than dry to wet. Finally, global correlations between wet-dry hydrological extremes and ENSO, PDO and AMO were also computed. Results to this end showed marked similarity between ENSO and PDO spatial patterns, with different results obtained for AMO. In conclusion, there is hope that these findings, in particular the maps shown in Figure 5.2-5.3, if integrated for example into global forecasting products, may provide useful insights for disaster risk reduction agencies, stakeholders and (re)insurance companies with assets spread worldwide, for example in hydropower production (Ng et al., 2017; Turner et al., 2017) and/or crop yield (Leng and Hall, 2019; Xie et al., 2018; Zampieri et al., 2017).

In the following Chapter 6 a discussion on the findings obtained from research Chapters 3-5 is presented. Specifically, for each research chapter integrating themes focussed on multi-hazards will be explored and further details on the application of the findings provided, along with links to the wider literature. 


\section{Chapter 6}

\section{Discussion}

\subsection{Overarching theme}

The work here presented is composed of three research Chapters (3-5) focussed on concurrent hydroclimatic extremes, and their implications, from catchment to global scales (De Luca et al., 2017, 2019a, 2019c). The interactions between hydroclimatic extremes, or more generally natural hazards, is a new sub-field that is growing exponentially in terms of research output (Figure 6.1). Thus, the research community now appreciates that a more holistic (i.e. multi-hazards/compound event) approach towards the investigation of natural hazards and risks should be taken (AghaKouchak et al., 2018; Zscheischler et al., 2018). In this chapter, a general discussion on the overarching theme, i.e. concurrent hydroclimatic hazards, is first presented. Then, for each research chapter, specific considerations with respect to the topics investigated were drawn.

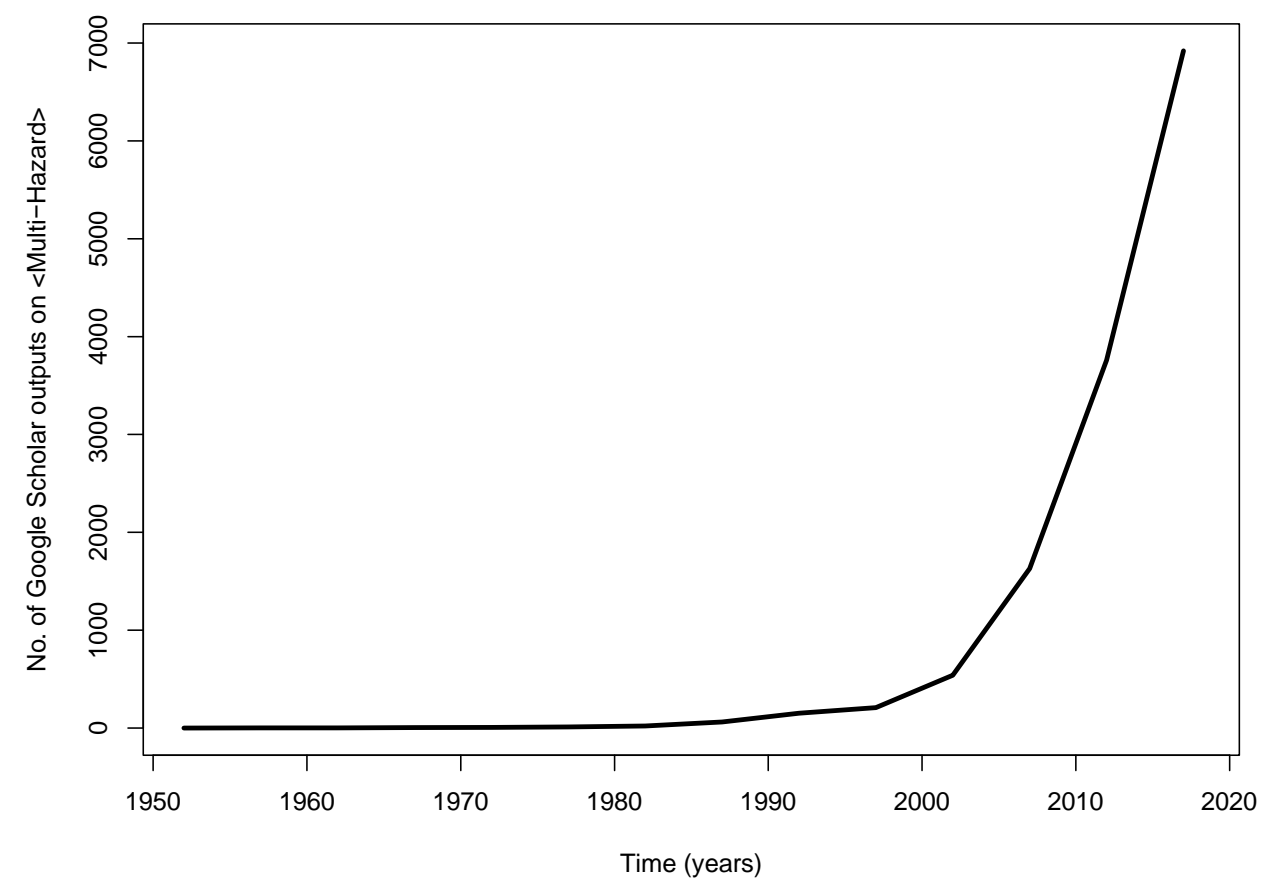

Figure 6.1 Annual number of Google Scholar outputs based on the keyword 'Multi-Hazard'. 
Natural hazards that can interact with each other and that are potentially affected by anthropogenic climate change are currently attracting significant attention from the scientific community, stakeholders, (re)insurance companies and emergency planners (e.g. Gill and Malamud, 2014; Kappes et al., 2012a; Koks et al., 2019; Leonard et al., 2014; Munich Re, 2015; UNDRR, 2017a). This is because their occurrence, strength and eventually socio-economic risks are set to likely increase in the future mainly because of a warming climate (IPCC, 2018, 2012). For example, Gill and Malamud (2014) provided a general overview of the relationships between 21 natural hazards, by identifying 90 different interactions and by emphasising the need of a multi-hazards approach towards natural hazard assessments. Similarly, Kappes et al. (2012a) outlined the current challenges for a multi-hazard and risk analysis, which are the choice of the approach (e.g. quantitative or semiquantitative), data and method availability, scale issues and the awareness of limitations. Among international organisations, the UN Sendai Framework for Disaster Risk Reduction (UNDRR, 2015) advocates a multi-hazard approach. Case-studies investigating concurrent hydroclimatic hazards are numerous. For example, national-scale analyses of flood and drought events in the UK (Collet et al., 2018; Visser-Quinn et al., 2019), compound dynamical and observed extremes over Europe and Eastern North America (De Luca et al., 2019b), global compound precipitation and wind extremes (Martius et al., 2016) and interactions between river and coastal flooding (Ward et al., 2018). All these studies can help in enhancing disaster risk reduction measures, by also bringing awareness of multi-hazards among the public and by providing socio-economic benefits from local to global scales.

From an environmental science perspective, natural hazards cannot always be considered as independent processes, because their occurrence, in time and space, is driven by physical variables that may, in full or in part generate other, different perils. For example, excessive rainfall may lead to flooding and trigger landslides, or tropical cyclones may generate both storm surges and fluvial flooding, whilst also impacting the built environment with severe winds. Thus, much research effort needs to be undertaken to assess the co-occurrence of these perils and not only because such events stimulate an interest, but for providing robust insights that eventually will be used by international organisations, governments and broadly-speaking stakeholders, to improve the socio-economic resilience.

It is clear that the scientific community is currently investing resources and time to better disentangle the common view of natural hazards as single processes (e.g. Gill and Malamud, 2014; Kappes et al., 2012a; Leonard et al., 2014). This is excellent news. However, the circle is not closed yet, because first of all there are still many research questions that need to be addressed (see for example Table 6.1) 
and secondly, all the body of research produced on multi-hazards must leave the not-always-pragmatic world of science to enter challenging policy landscapes. Hence, before seeing significant and concrete changes at the policy level it may take some time, years or possibly decades, as at present there still exist governments that clearly deny, for reasons that go beyond the scope of this work but that are purely economic-related, that the climate is changing, and that human-kind is the main cause. At present, the literature focussing on future climate projections of multi-hazards is not yet as numerous as that based on observations (e.g. Ben-Ari et al., 2018; Forzieri et al., 2016; Zscheischler and Seneviratne, 2017). Thus, there should be more efforts from the scientific community to addressing research questions that involve possible changes in frequency, magnitude and timing of multi-hazards into the future under a warming world. Since at present there exists a large availability of reanalysis products (e.g. C3S, 2017; Dee et al., 2011; Kalnay et al., 1996) and climate model data (e.g. Eyring et al., 2016; Taylor et al., 2011), constructing and validating future projections of multi-hazards, such as floods-drought, heatwaves-air pollution-drought, storm surges-river flooding and storms-floods, should become a priority for governments and stakeholders.

\section{Open Research Questions}

- How can global hot-spots of compound dynamical extremes be identified?

- How might multi-hazards events, such as concurrent floods and droughts, change in the future under a warming climate?

- What is the relationship between atmospheric rivers and storm surges?

- What are the possible links between Arctic Amplification and mid-latitude compound meteorological extremes?

- What are the physical mechanisms, if any, linking concurrent hydroclimatic extremes in spatially-remote regions?

- How might machine learning and satellite data help in forecasting multi-hazard events?

- What multi-hazards events have caused the largest socio-economic damages and why?

Table 6.1 Suggested open research questions within the field of multi-hazards.

A multi-hazard approach towards risk management (e.g. Kappes et al., 2012a; Terzi et al., 2019) will be extremely positive because the possibility of being for example insured to multiple concurrent perils within a given time window will enhance the chances of not suffering as much economic damages as 
for the single-hazard case. Multi-hazards research has also a fundamental value in scientific communication. Although local knowledge of a given territory plays an important role in managing natural risks, the general public may not always be aware that the occurrence of a given hazard may cascade or accumulate into a second or third peril. For example, a person could feel safe if he/she built his/her house outside of a floodplain, however he/she may have not considered that the mountain above the house is regularly subjected to landslides. In this case, heavy precipitation events, due to storms or thunderstorms, lasting for a sufficient amount of time will eventually saturate the soil on the slopes of the mountain and trigger landslides. Thus, local authorities have the duty to first gain knowledge, through for example multi-hazard (risk) assessments, about possible combined perils affecting the area and secondly, to inform residents about the risks via for example social media, scientific events, newspapers and tv news. Furthermore, past extreme events could also be used as analogues for future hazards (Matthews et al., 2016a).

\subsection{Research contributions in context}

With this thesis, several new insights on concurrent hydroclimatic hazards that can have significant socio-economic impacts are highlighted.

In Chapter 3, interactions between observed multi-basin flooding and extra-tropical cyclones (ETCs) in Great Britain (GB) are quantified and discussed (De Luca et al., 2017). First of all, a simple and pragmatic method for identifying widespread flooding is presented and this, along with similar recent studies (Berghuijs et al., 2019; Uhlemann et al., 2010), extends the conventional view of flooding that is limited to a single river basin giving, for example, the opportunity to emergency managers to estimate a national-scale return period of flooding. Secondly, multi-basin flooding in GB is found to be linked with ETCs over a time-window of $\sim 2$ weeks. The research also used and introduced new metrics, such as the F-Index (Wilby and Quinn, 2013), the (multi-basin) Flood-Yield and diverse sources of data that captured different flood information. In the concurrent extremes scenario introduced here, the risk of suffering socio-economic damage increases compared to a single hazard case, because not only can a property be flooded, but the same structure may also be damaged by severe winds. Such concurrent, widespread flood-wind impacts can therefore be also a cause of significant stress to emergency services and insurance companies, if reasonable and adequate multihazards prevention strategies are not deployed. For example, during a multi-basin flood coupled with severe winds, more than one Environment Agency (EA) areas may be severely hit. Hence, the supply 
chain for disaster relief needs to be able to provide enough support in diverse parts of the country, through cross-collaboration and sharing of human and logistical resources.

In Chapter 4, an analysis of past and projected seasonal frequency and persistence of weather patterns in the British Isles (BI) (Jenkinson and Collison, 1977; Jones et al., 1993; Lamb, 1972), along with associated multi-hazards is presented (De Luca et al., 2019a). From the results obtained, one can consider the future occurrence and related risks of two different cases of concurrent hydroclimatic hazards. These are concurrent flood-wind events during winter and concurrent heatwave-air pollution episodes in summer, with the latter focussed on London (UK). The weather patterns associated with such multi-hazards scenarios are the cyclonic (C) and westerly (W) types for flood-wind impacts (e.g. De Luca et al., 2017; Pattison and Lane, 2012) and the anticyclonic (A) type for heatwaves-air pollution and drought events (e.g. Coumou et al., 2018; O'Hare and Wilby, 1995; Pope et al., 2015; Wilby, 2008). Quantification of such concurrent hazards was achieved using two metrics, namely the F-Score, an extension of the F-Index used in De Luca et al. (2017) and the nocturnal Urban Heat Island (UHI) adapted from Wilby et al. (2011). Projected changes in the frequency and persistence of weather patterns, along with their connected multi-hazards, can be indicative of future climate change impact assessments and the methodology can be readily transferred to other mid-latitude regions and beyond (e.g. Cortesi et al., 2013; Donat et al., 2010b; Grundström et al., 2015b; Lorenzo et al., 2008). Sensible regions where the approach could be applied are for example the whole of Europe and its major cities, where there is already concern about heat-air quality hazards (Bastin et al., 2019).

In Chapter 5, the research focussed on globally observed concurrent wet and dry hydrological extremes and their association with modes of climate variability (De Luca et al., 2019c). Here, the most geographically widespread and concurrent flood and drought episodes are quantified and presented as maps. These findings provide new insights that are valuable for stakeholders and (re)insurance companies with assets spread worldwide and invested for example in hydropower ( $\mathrm{Ng}$ et al., 2017; Turner et al., 2017) or crop (Leng and Hall, 2019; Xie et al., 2018; Zampieri et al., 2017) production. They could also benefit road and railway infrastructures, as it is shown that globally about $27 \%$ of these assets are exposed to at least one hazard, with billions of US \$ contributing to global annual expected damages (Koks et al., 2019). The proposed global maps could be, for example, integrated into a seasonal/sub-seasonal hydrological forecasting model, so that information about multi-hazards is made available to targeted businesses, emergency organisation and the wider public. Such new development could be achieved by building an open-source online forecasting tool, updated for example every $24 \mathrm{~h} 7$ days a week. On a similar line, multi-hazard hydrological forecasts could also 
benefit from information about modes of climate variability, as there is a general consensus that climate indices need to be incorporated, or used as predictors, within the models (Eden et al., 2015; Emerton et al., 2019).

As per the previous two Chapters (3-4), the study also introduced two new metrics able to capture information with respect to global wet and dry hydrological extreme observations, namely the WetDry (WD) ratio and the Extreme Transitions (ET). The former informs about whether there was a predominance of extreme wet or extreme dry observations in the past, whereas the latter quantifies the time-lag needed to pass from extreme wet (dry) to extreme dry (wet) conditions over a given area. Overall, these results and metrics can also help emergency managers and governements, of geographycally-large countries (e.g. USA, Russia and China), to better prepare and increase resilience towards spatio-temporally concurrent flood and drought events. For example, future climate projections of the WD-ratio up to 2100 could reveal the propensity of a given area to experience more wet or dry extremes. Such information may be highly valuable, as stakeholders investing in irrigation infrastructure or hydropower generation can adapt their long-term portfolio accordingly to the maps. For example, if future WD-ratio projections show a region shifting from more dry to wet extremes, farmers and stakeholders could consider investing infrastructures and crops' resources in that area. On the other hand, a projected shift from more wet to dry extremes can stop stakeholders from investing in the given region. Similarly, future projections of the ET time-lag can benefit emergency managers and broadly disaster risk reduction procedures, as knowing the time between for example a severe flood and drought would be highly valuable for planning for example the disaster cycle phases (i.e. response, recovery, mitigation and preparation).

\subsection{Summary}

This chapter provides a discussion of the overarching theme and contributions of each chapter, in a wider context. Multi-hazards research has expanded considerably over the last decade, as the research community realise that natural hazards can interact over time and space, and that their co-occurrence may lead to more significant socio-economic damages than the single hazard components (Gill and Malamud, 2014; Zscheischler et al., 2018). The multi-hazards subject also started to receive attention from international organisations (UNDRR, 2017a) and there is hope that its importance will be also soon be extended at the national, regional and local levels. However, for doing so, a significant shift 
in legislations and regulations, expanding the common view of natural hazards to the possibility that such events can develop into multi-hazards, must be made and if so, it will not be immediate.

A multi-hazards approach can be valuable for a different set of stakeholders and communities, such as (re)insurance companies, emergency managers and environmental protection agencies. Concurrent flood-wind hazards are somehow expected in GB, as the country is situated under the north Atlantic storm-track. The results showed in Chapters 3-4 proved the existence of this association, along with its enhanced occurrence in the future within a warmer world. Hence, such flood-wind scenario needs to be considered by for example the government, EA and (re)insurance companies. The nocturnal UHI temperatures for London and anticyclonic persistence and frequency in the BI (Chapter 4), during summer, are also set to increase by the end of the 21 st century. This could lead to an increased risk of concurrent heatwave-air pollution-drought events, that can put under high stress the society and the emergency management services. Lastly, in Chapter 5 the co-occurrence of wet and dry hydrological extreme events is shown, along with documented impacts of the most geographically widespread ones. These findings may help global stakeholders in hedging economic losses and governments of large countries, that can experience both opposite extremes within the same time-frame.

In the next Chapter the conclusions will be drawn from each research chapter and overarching theme, along with overall final remarks. Specifically, each chapter will be summarised, the research questions revisited, future work directions presented, and the significance of the research highlighted. The concluding remarks will focus on the hope for more urgent actions about anthropogenic climate change, how this thesis fits into this landscape and how multi-hazards research can contribute with respect to the climate issue. 


\section{Chapter 7 \\ Conclusions}

This thesis examined concurrent hydroclimatic hazards from catchment to global scales. Different metrics were deployed for analysing the intereactions between hazards, with potential benefits for stakeholders, policy makers and emergency managers. The topics investigated were combined widespread flood-wind episodes (Chapter 3-4), future projections of weather pattern persistence and associated multi-hazards (Chapter 4) and concurrent wet-dry hydrological extreme events (Chapter 5). The time periods, covered observations spanning from 1950 to 2014, as well as climate model historical runs for the observational period 1970-2000 and the future from 2011 to 2100. The geographical domains under investigation were Great Britain (GB), the British Isles (BI) and the whole globe. This chapter reprises the key literature gaps and related research questions, main findings of this thesis, a discussion of the unifying theme and possible ways forward (Sections 7.1-7.3). Then, a paragraph about the need for urgent actions about anthropogenic climate change can be found in Section 7.4, before the very last Section 7.5 that draws out the general conclusion on the overarching theme and how multi-hazards research could be integrated into anthropogenic climate change studies.

\subsection{Concurrent flood-wind hazards}

Chapter 3 reports the results of a multi-hazards study on observed extreme multi-basin flooding linked with extra-tropical cyclones (ETCs) impacting GB (De Luca et al., 2017). The identified literature gap was that river flooding if often investigated as a single-basin event, without considering the possibility that within the same flooding episode, multiple basins can concurrently flood within a given window of several days. At the time the research was started, only one study (Uhlemann et al., 2010) was found addressing this research question. Chapter 3 advanced this work by adding a simple and pragamatic methology, using annual maximum (AMAX) peak river flows, for extracting multi-basin flooding events within a selected time-window of several days. Subsequently, Berghuijs et al. (2019) extended the topic with a European assessment of synchronous flooding. However, the main literature gap was that these widespread floods were not found to be associated with ETCs impacting GB, and that this can eventually lead to a multi-hazards scenario of combined flood and wind socio-economic impacts. 
The main research questions addressed in Chapter 3 were the following: i) What is the spatio-temporal distribution of multi-basin flooding episodes? ii) What are the most frequent weather patterns observed during these widespread floods? and iii) How are multi-basin floods, atmospheric rivers (ARs) and very severe gales (VSGs) linked?

The results showed five extreme multi-basin flooding episodes within the 1975-2014 period and in a time-window of 1 to 19 days. These matched with existing literature reporting widespread socioeconomic impacts across GB due to flooding. The peak flow annual maxima (AMAX), used as a proxy for flooding, impacted between 66 to 108 basins and a total drained area (TDA) of $19 \%$ to $47 \%$ of the study area during a window of 1 to 19 days. These events occurred during winter, with a frequency peak in January and most common LWTs were the cyclonic (C) and westerly (W). Wet soil moisture conditions, measured via the standardized precipitation index (SPI) (McKee et al., 1995; 1993) preceded some of the episodes. Then it was also found that atmospheric rivers (ARs) (Brands et al., 2016; Lavers et al., 2011) and VSGs (Jenkinson and Collison, 1977; Jones et al., 1993) occurred within the same days or before the extreme multi-basin flooding episodes. Such findings, combining multibasin flooding with C-type weather patterns, ARs and VSGs established for the first time a compound association between floods and ETCs (De Luca et al., 2017).

The methods and findings for extreme multi-basin flooding and ETCs have the scope to be extended to other regions. Possible ways forward could be to upscale the domain of study to the whole of Europe, USA or Canada as well as to conduct future hydroclimatic projections, for example through statistical downscaling (Wilby and Wigley, 1997), under different Representative Concentration Pathways (RCPs). Lastly, extreme value theory (EVT) (Coles, 2001) can provide insights into the statistical coupling of both flooding and wind extreme values. This could be achieved by, for example, fitting a multivariate extreme value model (e.g. Heffernan and Tawn, 2004), or simply by performing correlation tests, between peak river flows and wind gust observations recorded in the same areas. Specifically, one could select a network of river basins, covering a sufficiently large region (e.g. a country or continent) and link each of these with wind gusts recorded from the closest meteorological station.

Several benefits emerge from the research in Chapter 3. First, is the introduction of a new simple and pragmatic method to quantify the temporal and spatial extent of multi-basin flooding episodes. Such approaches can theoretically lead to the estimation of a national-scale return period of widespread flooding, that will be useful to emergency managers and planners. This method is transferrable beyond 
$\mathrm{GB}$, although attention needs to be given to catchment area, as drainage networks within extremely large basins may not necessarily be flooded entirely and within the same time-period as small catchments. Second, it is found that multi-basin flooding events can cause socio-economic damages that in some cases go beyond what is planned for by emergency agencies and affect local communities and businesses (De Luca et al., 2017). Third, such damages can be amplified by coupled severe wind impacts. The results expose a compound risk that needs to be covered by (re)insurance companies, emergency managers and stakeholders.

\subsection{Weather pattern persistence and multi-hazards}

Chapter 4 investigated observed and future seasonal climate projections of Lamb Weather Types (LWTs) (Jenkinson and Collison, 1977; Jones et al., 1993; Lamb, 1972) frequency and persistence within the BI and their links with multi-hazards (De Luca et al., 2019a). The main literature gap was the lack of studies linking weather patterns to multi-hazards, such as flood-wind and heatwave-air pollution-drought events. Moreover, no previous research has brought this topic into light by performing climate model evaluation and projections up to 2100.

The specific research questions addressed by Chapter 4 were the following: i) How has persistence in weather pattens changed historically? ii) To what extent can Atmosphere-Ocean General Circulation Models (AOGCMs) reproduce observed weather pattern persistence over the BI? iii) How are weather pattern persistence and frequency expected to change in the future under different Representative Concentration Pathways (RCPs)? and iv) How migth variations in future weather type persistence translate into changed risk of winter flood-wind and summer heatwave-air pollution concurrent hazards?

The results showed that AOGCMs are generally capable of reproducing weather pattern seasonal persistence when compared with NCEP (Kalnay et al., 1996) and 20CR (Compo et al., 2011) reanalyses products, across the historical 1971-2000 (1980s) period. Persistence for the anticyclonic (A) type is projected to increase in the future during summer; cyclonic (C) weather on the other hand decreases in all seasons and the westerly (W) type does not change in winter, but increases in autumn and decreases in spring under RCP8.5. Moreover, the frequencies of A- and W-types are projected to increase significantly by 2100 during summer and winter respectively (RCP8.5, p-value <0.01). Whereas C-type frequency during autumn is projected to decrease during autumn ( $\mathrm{p}$-value $<0.01)$. 
With respect to multi-hazards, two metrics, the F-Score and the nocturnal Urban Heat Island (UHI) temperatures, were computed respectively for concurrent winter flood-wind and summer heatwave-air pollution events. Results showed that the F-Score is overestimated by the AOGCMs when compared to NCEP, 20CR reanalyses and Lamb's subjective dataset. However the risk of concurrent flood-wind events is set to significantly increase by 2100 . On the other hand, the nocturnal UHI temperatures for London (UK) are slightly underestimated by AOGCMs, and as per the F-Score, severity is projected to significantly increase by 2100 .

Further possible research on the topic could be undertaken by applying the same persistence and frequency method to other regions with established weather pattern typologies, such as the Grosswetterlagen for Europe (Hess and Brezowsky, 1952) and Spatial Synoptic Classification for North America (Kalkstein et al., 1996). The analysis could also be extended by making use of a larger ensemble of both reanalyses and AOGCMs, and by investigating other types of multi-hazards scenarios, such as concurrent river flooding-storm surges and/or flood-drought events.

The findings for the A-type weather pattern may signify an increased chance of blocking episodes which could increase the risk of poor air quality, droughts and heatwaves during summer (Coumou et al., 2018; Munich Re, 2015; Tang et al., 2013). These changes could be linked to Arctic Amplification (AA) (Screen and Simmonds, 2010) but are difficult to reconcile with a projected decrease in blocking events over the BI during summer by 2061-2099 under RCP8.5 (Woollings et al., 2018). A decrease in persistence and frequency for the C-type during autumn can imply reduced chance of extreme precipitation and flooding, linked to ETCs impacting the BI (De Luca et al., 2017); whereas a more frequent W-type (or zonal flow) during winter can bring high rainfall events and possible fluvial flooding (Pattison and Lane, 2012). Results for multi-hazards suggest that by the end of the 21st century, there could be an increasing chance of concurrent flood-wind hazards during winter and concurrent heatwave-air pollution hazards during summer (in London). These findings bring new insights into future projections of concurrent climate extremes in the BI. They can be valuable for governments when it comes to increasing resilience against concurrent hydroclimatic extremes. Finally, the methodology can help in both evaluating AOGCMs realism as well as providing narratives of future hydroclimatic risks that can be intelligible to a wide range of user communities. 


\subsection{Concurrent wet and dry hydrological extremes}

Chapter 5 examined concurrent wet and dry hydrological extremes at the global scale and their relationship with modes of climate variability (De Luca et al., 2019c). The literature gaps filled by the chapter were (a) the lack of a global observational analysis of spatio-temporal joint occurrences of extreme wet and dry events, (b) metrics to investigate such opposed extremes and (c) studies showing how these extremes are linked with predominant climate indices.

The research questions were the following: i) How have observed globally independent and concurrent wet-dry hydrological extreme events changed in the past? ii) What were the most spatially extensive independent and concurrent wet-dry hydrological extreme events? iii) How might new metrics shed light into the likelihood of concurrent wet-dry extremes? and iv) How are these extremes related to different modes of climate variability?

The results presented in Chapter 5 contribute to a deeper understanding of concurrent wet and dry hydrological extremes. First, it is shown that the global land area impacted by dry and wet-dry extreme events significantly increased during the period 1950-2014. The most geographically widespread wet, dry and wet-dry events coincided with well known flooding and drought episodes around the globe. Second, the two metrics developed (namely the Wet-Dry, WD ratio and the Extreme Transitions, ET) provided insights into the past predominance of wet or dry extremes in a given area and about the globally aggregated time-lag between a wet (dry) and dry (wet) extreme observation respectively. The WD-ratio identified regions that experience more wet than dry extremes as the USA, northern and southern South-America, northern Europe, North Africa, western China and most of Australia. The remaining regions, on the other hand, show a predominance of dry extremes. Third, the ET between wet to dry are on average significantly slower than the ones from dry to wet. Finally, global correlations between climate indices and wet and dry extremes showed that the El Niño Southern Oscillation (ENSO) and Pacific Decadal Oscillation (PDO) have patterns of positive correlations with wet extremes over southern and western USA, south Brazil and Argentina and the middle-east. Conversely, significant negative correlations were found over north-western North America, northern Brazil, central and southern Africa, eastern Russia, Indonesia and eastern Australia. On the other hand, the Atlantic Multidecadal Oscillation (AMO) generally exhibited opposite correlation patterns when compared to ENSO and PDO. 
These analyses could be extended in different ways, so that a more profound understanding of the joint spatio-temporal occurrences of global wet and dry extremes, can be achieved. For example, Chapter 5 made use of the self-calibrated Palmer Drought Severity Index based on the Penman-Monteith model (sc_PDSI_pm) (Dai, 2017; Sheffield et al., 2012). Thus, there is scope for replicating the work by using other global hydrological datasets such as the Standardized Precipitation Index (SPI) (McKee T.B., Doesken N.J., 1995; McKee et al., 1993) or the Standardized Precipitation Evapotranspiration Index (Vicente-Serrano et al., 2010). This would also reveal the sensitivity of the present findings to the choice of hydrological data. Moreover, analysing concurrent seasonal wet and dry extremes may also be relevant, as stronger spatio-temporal patterns may emerge when compared to the annual timescale. The wet-dry extremes could also be differentiated by Köppen climate zones (Rubel and Kottek, 2010), so that their variations can be objectively assigned to specific regions of the world. Lastly, once solid observational results are established, the next step would be to perform AOGCMs simulations and project future changes of concurrent wet and dry hydrological extremes for a warming world.

The findings in Chapter 5, on the spatio-temporal co-occurrence of wet and dry hydrological extremes, are relevant to stakeholders with global assets and/or supply chain. Thus, understanding the behaviour of future concurrent wet and dry extremes, through maps of the most widespread events and future projections of the WD-ratio, can help for example in hedging economic losses with respect to regional hydropower production (Ng et al., 2017; Turner et al., 2017), crop yields (Leng and Hall, 2019; Xie et al., 2018; Zampieri et al., 2017), planting and harvesting schedules (Sacks et al., 2010), and global transport networks (Koks et al., 2019). Furthermore, future projections of the ET would be useful for improving disaster risk reduction measures, through an enhancement of resilience.

\subsection{The climate is already changing, what about us?}

It is apparent that citizens and governments are becoming more aware of the greatest problem humankind is currently facing, i.e. anthropogenic climate change and its related socio-economic impacts due to weather and climate extremes (IPCC, 2018, 2012; UNDRR, 2015). As a consequence, many countries, such as Sweden, Iceland, Norway and Ireland are investing heavily in renewable energy resources. Thus, there is great hope among these leading countries that the socio-economic impacts of climate change can be significantly mitigated, so that future generations will be able to continue living a decent and sustainable life. Many things however, still need to change, as at present there is still a lack of general consensus among all the governements about the fact that the climate is changing and 
that we, as human-kind, are the main cause of the issue. Such a thing is something that needs to be addressed and fixed as soon as possible, because at the moment some of the countries denying climate change (e.g. USA, Brazil, Russia and Saudi Arabia) are among the largest greenhouse gas producers.

The world at present really needs top-down and bottom-up political actions against climate change and its influence on weather and climate extremes, so that fossil fuels will be replaced with renewable energies, significant investment in research tackling extreme events will be made, sustainable adaptation measures will be deployed and a green and sustainable economy will be incentivised. It is also clear that leaders who refuse or delay to act against climate change are not doing so because they do not realise the gravity of the issue, or because they are wrongly advised by the scientific community, but simply because short-term economic interests do not allow tackling climate change. It is also true that if each person in the world does a little to reduce his/her carbon footprint the advantage for us can be enormous (e.g. mantaining a vegetarian diet, reducing the electric consumption at home, buying electric cars, etc..). However, the truth is that the greatest changes towards a greener planet need to come from governments and politicians, as they are the only ones heard by the people.

Thus, a possible way forward, which at the moment is definitely growing with importance and taking place around the world, is to educate and sensitise the old but in particular the young generations about the fact that: i) climate change is currently happening; ii) climate change is having a significant effect on exacerbating the magnitude and frequency of weather and climate extremes, with even more devastating socio-economic impacts; iii) if actions are not taken in full, the outcomes may lead to irreparable damages to human-kind; and iv) that solutions to tackle the problem currently exist, but they can only materialise with targeted political choices and actions. The climate on earth has always changed and it will continue to do so with or without our presence. Human-kind represents only a tiny and possibly insignificant temporal breath over the entire earth's life. The earth will definitely survive this current anthropogenic climate change, but will we survive stronger and more frequent weather and climate extremes? It is therefore in the best interest of all people on earth to accept the scientific consensus about climate change and to urge for more actions, not because we will be able to see any positive difference in our lifetime, but because we will be able to donate to future generations a world that is still able to provide a livable environment. 


\subsection{Concluding remarks about multi-hazards}

There is hope that this thesis on concurrent hydroclimatic hazards highlights the importance of continuing investment of time and resources into research focussed on the understanding of concurrent weather, climate and hydrological hazards and risks. More research is indeed required in this field and it also needs to be translated by policy makers and stakeholders, into concrete solutions and actions that can eventually enhance socio-economic resilience to extreme events at the global, regional, national and local scales. In particular, since a new sub-field of research focussing on multi-hazards, or compound events, (e.g. Gill and Malamud, 2014; Zscheischler et al., 2018) has been now established, new research questions arise along with the need to put into practice the findings coming from the research community. Natural hazards that are driven by the atmosphere (e.g. floods, droughts, heatwaves and storms) are likely to become more severe in the future due to anthropogenic climate change (IPCC, 2018, 2012). This statement is just one of the many highlighting the immediate need of pragmatic solutions to better prevent, adapt to and better predict concurrent weather, climate and hydrological hazards and associated impacts.

At present, despite a growing body of literature reviewing multi-hazards (e.g. Gill and Malamud, 2017, 2014; Kappes et al., 2012b, 2012a; Leonard et al., 2014; Terzi et al., 2019; Tilloy et al., 2019; Zscheischler et al., 2018) and numerous studies investigating diverse interrelationships between the perils (e.g. Collet et al., 2018; De Luca et al., 2019b; Koks et al., 2019; Martius et al., 2016; VisserQuinn et al., 2019; Ward et al., 2018), very little has been done with respect to future climate projections of compound events (Ben-Ari et al., 2018; Forzieri et al., 2016; Zscheischler and Seneviratne, 2017). Thus, a general way forward within the field of multi-hazards would be indeed to build international projects, between research institutes, universities, companies and policy makers, focussed on future scenarios of multi-hazard events under a warming world.

Such research, could focus on hazard interactions including: i) future projections and links to the Arctic Amplification (Screen and Simmonds, 2010); ii) global assessments of future socio-economic damages from multi-hazards; iii) the discovery of new physical multi-hazard processes (e.g. atmospheric rivers driving storm surges and river flooding); iv) spatio-temporal shifts in the distribution of multi-hazards and v) the application of multivariate extreme value statistics (e.g. Heffernan and Tawn, 2004) and dynamical systems theory (De Luca et al., 2019b; Faranda et al., 2017b, 2017a; Hochman et al., 2019; Lucarini et al., 2016, 2012; Messori et al., 2017; Rodrigues et al., 2018) to climate projections (Faranda et al., 2019). Such research efforts could also focus on different spatial scales, from cities, to countries, 
continents and global areas. They should also involve multi-disciplinary teams, spanning the social sciences, philosophy, mathematics, physics, computer science, geography and engineering.

In conclusion, this thesis analysed different sets of concurrent hydroclimatic hazards, from catchment to global scales using both observations and climate model projections. It is shown that in Great Britain extreme multi-basin flooding is linked to extra-tropical cyclones. The work also reveals that concurrent flood-wind and heatwave-air pollution-drought hazards could increase in the future, and that substantial areas of the world can be impacted by concurrent yet geographically-remote flood and drought events. This matters because, apart the fact that such multi-hazard events are extremely fascinating per se, they can generate enormous socio-economic damages, which should be understood and managed. 


\section{Annex 1}

\section{A.1 Supplementary Information Chapter 3}

\section{A.1.1 Figures}

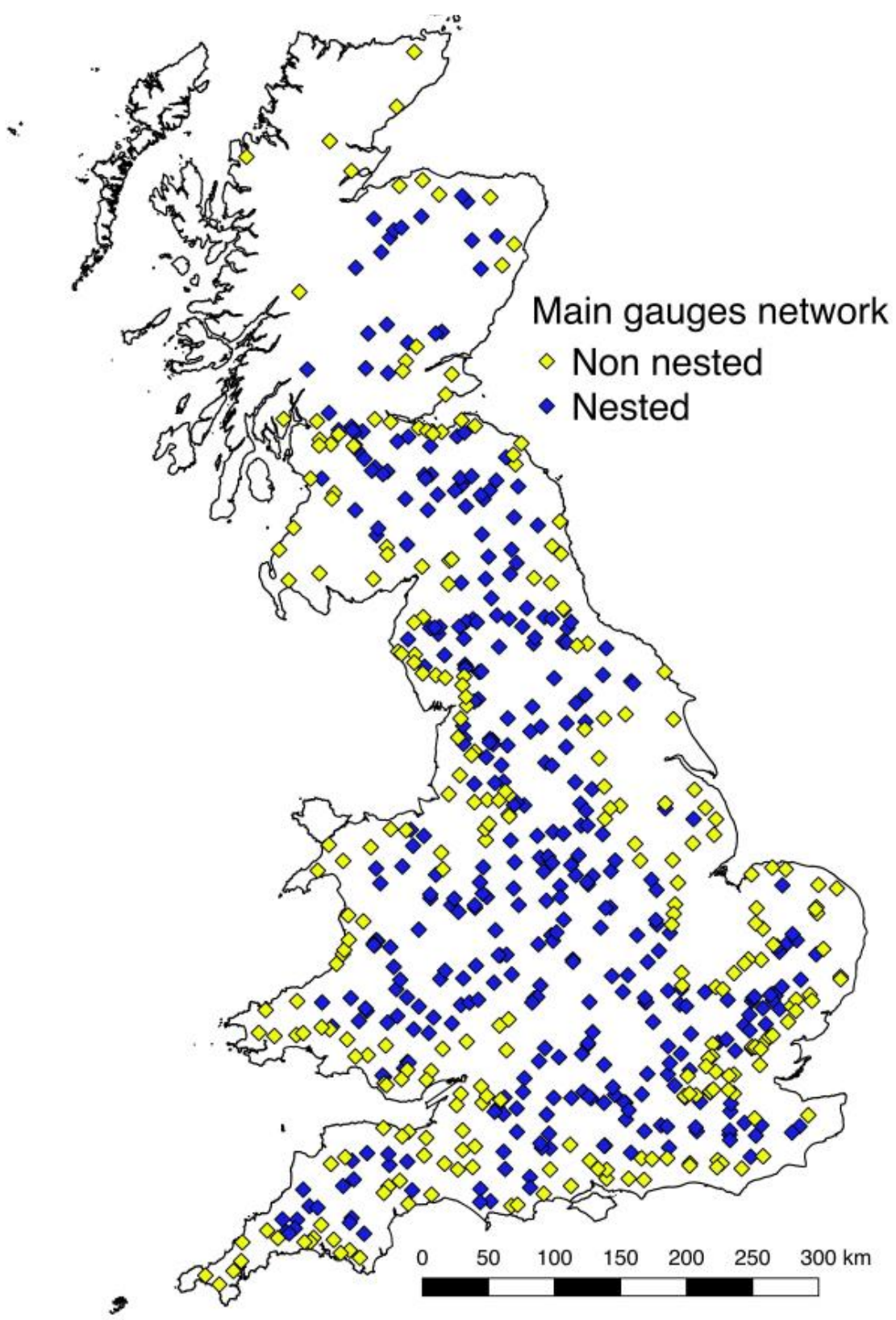

Figure S3.1 Initial hydrological network of 649 gauges. The yellow stations are the 261 non-nested basins used in the analyses, whereas blue stations represent the remaining 388 nested stations excluded from the study because they are located upstream from a non-nested gauge. 

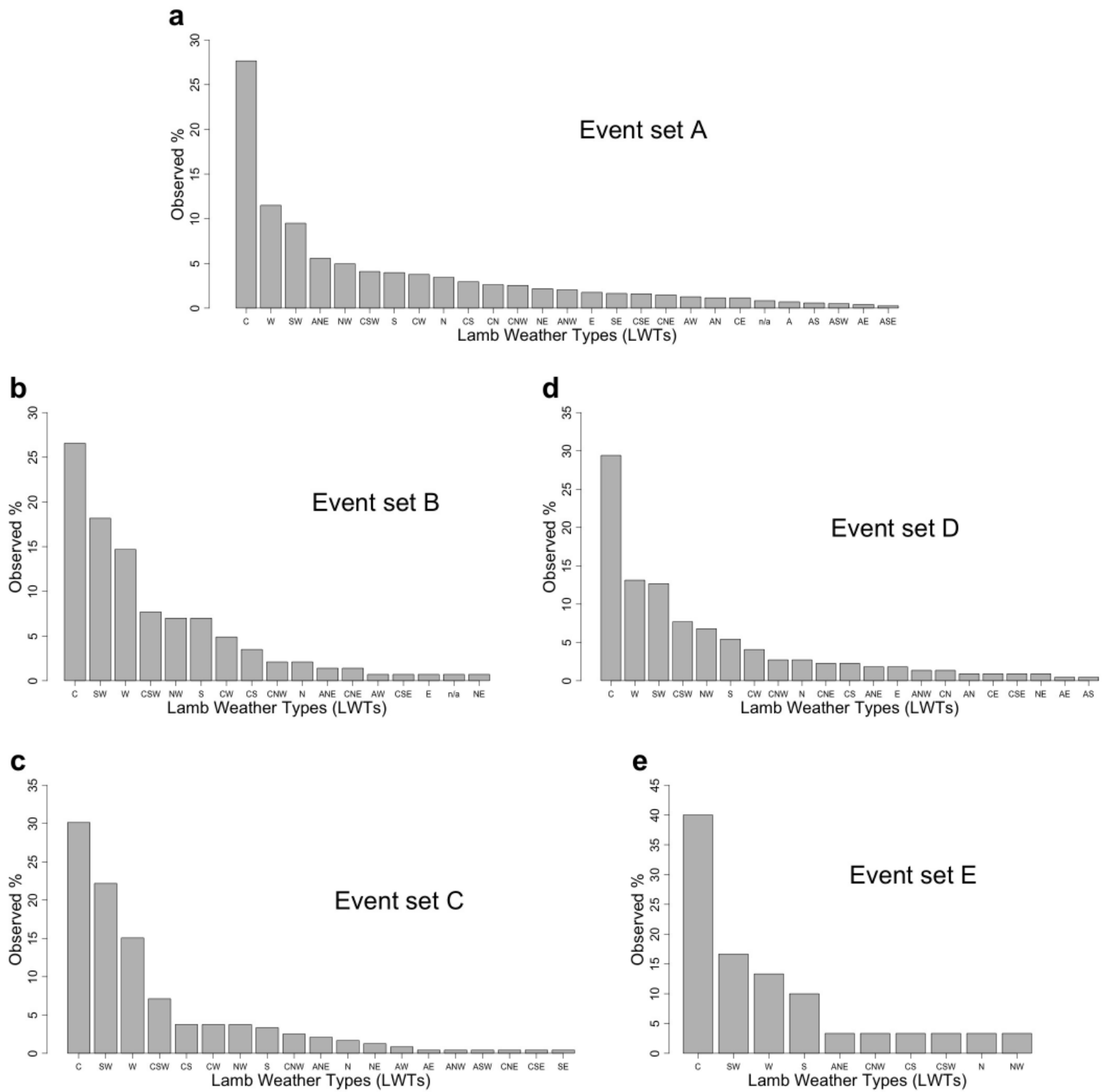

Figure S3.2 Lamb Weather Types' (LWTs) observed percentages of occurrence for all event sets (A-E). (a) Event set $A ;(b)$ event set $B ;(c)$ event set $C ;(d)$ event set $D ;$ and $(e)$ event set $E$. All with replicated dates excluded. 


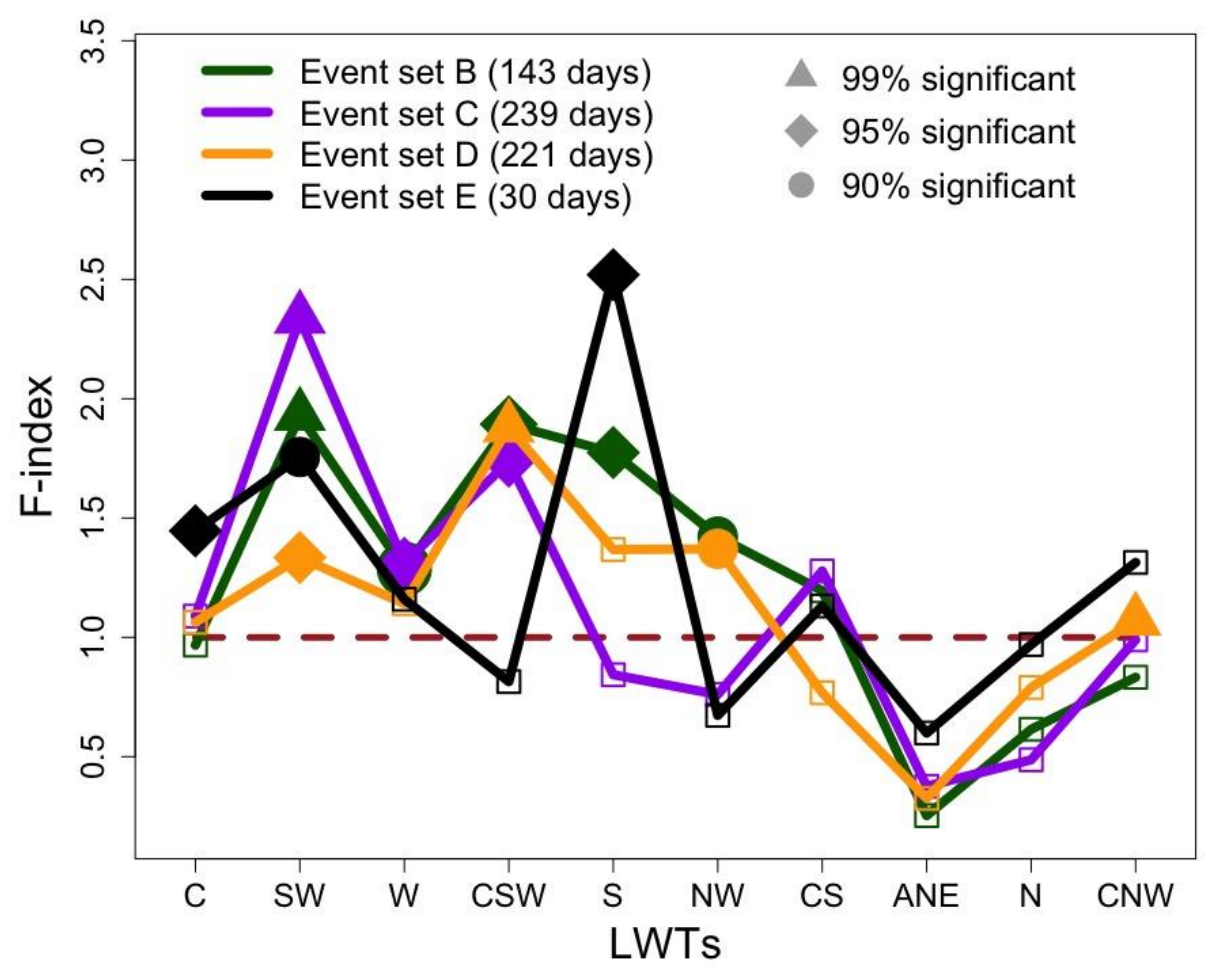

Figure S3.3 Lamb Weather Types' F-Index (Wilby and Quinn, 2013) calculated for event sets B, C, D and E with respect to single-basin occurrences (i.e. event set A). Significance was determined using Binomial test, but with event set $A$ used as expected values. LWTs shown are based on event set E; event sets $B-D$ also contain other LWTs. 

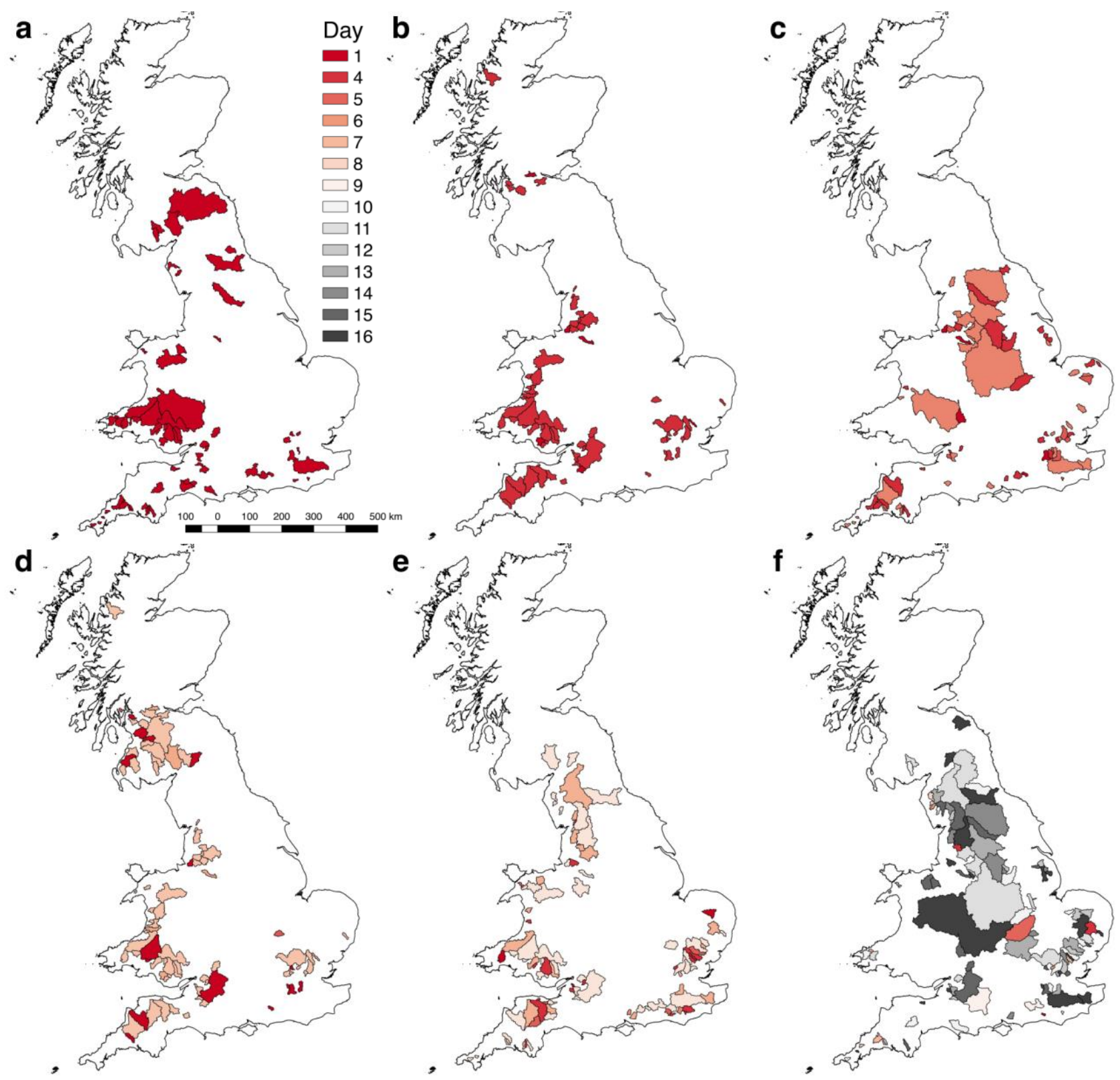

Figure S3.4 Extreme multi-basin flooding episodes' joining times (event set E). (a) $L=1$-day (dmax $=$ 27/12/1979); (b) L=2-days $\left(d_{\max }=30 / 10 / 2000\right) ;(c) L=4$-days $\left(d_{\max }=01 / 01 / 2003\right) ;(d) L=6$-days $\left(d_{\max }=\right.$ 30/10/2000); (e) $L=8$-days $\left(d_{\max }=02 / 12 / 1992\right) ;$ and $(f) L=16$-days $\left(d_{\max }=01 / 02 / 1995\right)$. Days are ordered chronologically (e.g. Day $=16$ represents $d_{\max }$ for $L=16$-days). 


\section{Annex 2}

\section{A.2 Supplementary Information Chapter 4}

\section{A.2.1 Methods}

\section{A.2.1.1 CMIP5, reanalyses and Lamb's catalogue}

The climate model output used to represent historical, future Representative Concentration Pathway 8.5 (RCP8.5) and RCP4.5 projections of LWTs (Jones et al., 1993; Lamb, 1972) originate from a multi-model sub-ensemble (MME) of 10 Atmosphere-Ocean General Circulation Models (AOGCMs) from the Coupled Model Intercomparison Project Phase 5 (CMIP5) (Taylor et al., 2011). MME output was obtained from the Earth System Grid Federation (https://esgf-node.llnl.gov/search/cmip5/). Per each model the historical, RCP8.5 and RCP4.5 runs of daily (12 UTC) sea-level pressure (SLP) are used to calculate daily LWTs across the BI as described above. The historical period is defined as 1980s (1971-2000). Model runs for the RCPs (2006-2100) are divided into consecutive 30-year periods covering the 2020s (2011-2040), 2050s (2041-2070) and 2080s (2071-2100). Each AOGCM was regridded to $5^{\circ} \times 10^{\circ}$ (latitude $\times$ longitude) to match the grid of the objective LWT classification (Jenkinson and Collison, 1977; Jones et al., 1993). The choice of AOGCMs was constrained by availability of daily SLP for historical, RCP8.5 and RCP4.5 runs. Table 4.1 lists these models along with some of their characteristics.

To evaluate CMIP5 MME realism, LWTs were derived from two reanalyses (Jones et al., 2013) then compared with the 30-year historical (1980s) run of the MME. These were the 20CR (Compo et al., 2011) and NCEP (Kalnay et al., 1996) LWTs datasets (Jones et al., 2013), available from https://crudata.uea.ac.uk/cru/data/lwt/. In addition to reanalyses, a comparison of the historical MME with Lamb's catalogue of subjectively defined LWTs (Hulme and Barrow, 1997; Lamb, 1972), which ends in 1997, is also provided. The MME realism is evaluated using LWTs occurring in four seasons, namely: summer (June-July-August, JJA); autumn (September-October-November, SON); winter (December-January-February, DJF); and spring (March-April-May, MAM). Seasons were assigned to the year with the first month (e.g. summer 2020 includes June 2020, July 2020 and August 2020, whilst winter 2000 includes December 2000, January 2001 and February 2001). Note that for Lamb's catalogue, DJF for the 1980s has December and January for winter 1996, because the dataset ends in early February 1997. All the complete LWTs datasets used in the analyses are provided in the csv files accompanying this study. 


\section{A.2.1.2 Statistical methods and analyses}

\section{A.2.1.2.1 2-day persistence}

2-day persistence of LWTs (Jones et al., 1993; Lamb, 1972) was derived from Markov-chain matrices of transitions between the eight main weather types defined above (Gagniuc, 2017; Wilby, 1994). Persistence was defined as the probability that a given LWT on day $(t)$ is followed by the same LWT on day $(t+1)$. LWTs persistence is calculated for each AOGCM and MME mean (MMEM) for historical 1980s and 2020s, 2050s, 2080s under RCP8.5 and RCP4.5. Uncertainty in persistence estimates for the CMIP5 MME 1980s was calculated by boot-strapping (n=1,000) 30-year simulations to obtain $95 \%$ confidence intervals for significance testing. Persistence for the 2020s, 2050s and 2080s was calculated from the transition matrices. Persistence analysis was performed using the functions markovchainFit and createSequenceMatrix, from the R package 'markovchain' (Spedicato, 2017), respectively for historical boot-strapping and the three future periods (https://cran.rproject.org/web/packages/markovchain/markovchain.pdf). To evaluate the performance of the CMIP5 MME, the 20CR (Compo et al., 2011), NCEP (Kalnay et al., 1996), and Lamb's subjective classification (Hulme and Barrow, 1997; Lamb, 1972) were also used to calculate LWT persistence during the 1980s period. The CMIP5 MME historical persistence for 1971-1996 (not shown here) is also computed, to test the slightly shorter period covered by Lamb's subjective catalogue. After performing a Mann-Whitney-Wilcoxon two-tailed test (Mann and Whitney, 1947) (null hypothesis of no difference in mean persistence), between the MME 1971-1996 and MME 1980s, for A, C, W, and S LWTs within respectively summer (JJA), autumn (SON), winter (DJF) and spring (MAM), it is found no statistical significance between the two periods. Therefore, it is possible to conclude that Lamb's catalogue is equivalent to the 1980 s, despite being 5 years shorter.

The seasonal persistence for each LWT, AOGCM, MMEM, 20CR, NCEP and Lamb's subjective catalogue during the 1980s and (for AOGCMs only) 2020s, 2050s and 2080s under RCP8.5 and RCP4.5 are provided in the spreadsheets accompanying this study.

The statistical significance of changes in persistence for each LWT was assessed by testing: (i) differences in the persistence of the MME between the 1980s and the 2020s, 2050s and 2080s; and ii) differences in the persistence for individual climate models in the MME. In the first case (i) the MannWhitney-Wilcoxon two-tailed test (Mann and Whitney, 1947) was applied, under the assumption that data are not normally distributed, with the null hypothesis of no difference in mean persistence (Tables 4.2-4.3). The second test (ii) was performed individually for each model by checking whether 
persistence in the 2020s, 2050s and 2080s falls outside the boot-strapped $95 \%$ confidence intervals of the 1980s (Figures 4.4-4.5).

\section{A.2.1.2.2 Seasonal trends}

Trend analysis was performed using annual series of LWT frequencies from 2006-2100 to detect both linear and non-linear changes in LWT frequencies within the CMIP5 (Taylor et al., 2011) MMEM under RCP8.5 and RCP4.5 scenarios. For the sake of brevity, only trends for anticyclonic (A, summer JJA), cyclonic (C, autumn SON) and westerly (W, winter DJF) are shown, as indicators of impactful weather in the BI and for southerly (S, spring MAM) as this is the LWT showing most significant changes in persistence with the Mann-Whitney-Wilcoxon two-tailed test (Mann and Whitney, 1947) (Tables 4.2-4.3). A modified Mann-Kendall test (Hamed and Ramachandra Rao, 1998), which takes into account possible autocorrelation within the time series, was applied to both RCP8.5 and RCP4.5 seasonal MMEM LWTs frequencies.

Results from the trend analysis are presented in Figures 4.6-4.7 and Table 4.4 in terms of time series and Sen's slope (Sen, 1968) with relative statistical significance (i.e. p-value of modified MannKendall test, Hamed and Ramachandra Rao, 1998). Shaded bands in Figures 4.6-4.7 represent the 95\% confidence interval of the MMEM. Sen's slope gives information about the gradient, with large Sen denoting rapid changes; the sign shows whether the trend is rising (+) or falling (-). Sen's slope values and relative statistical significance are shown in Table 4.4. 


\section{Annex 3}

A.3 The published article within the journal Environmental Research Letters Chapter 3 of this thesis 


\title{
Environmental Research Letters
}

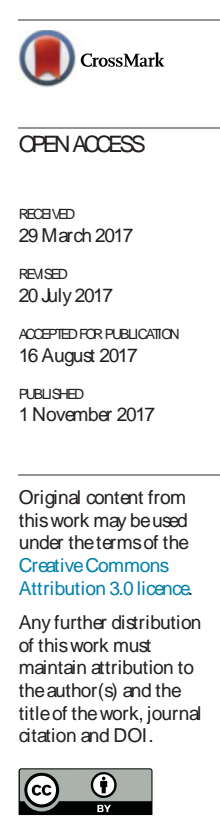

LETाषR

\section{Extreme multi-basin flooding linked with extra-tropical cyclones}

\author{
Paolo De Luca ${ }^{1,4} \mathbb{1}$, John K Hillier ${ }^{1}$, Robert L Wilby ${ }^{1}$, Nevil W Quinn ${ }^{2}$ and Shaun Harrigan ${ }^{3}$ \\ 1 Department of Geography - Loughborough University, Loughborough, United Kingdom \\ 2 Department of Geography and Environmental Management - University of theWest of England, Bristol, United Kingdom \\ 3 Centrefor Ecology \& Hydrology (CEH), Wallingford, United Kingdom \\ 4 Author to whom any correspondence should beaddressed. \\ E-mail:p.deluca@boro.ac.uk \\ Keywords: multi-basin, flooding, extra-tropical cyclones, Great Britain, emergency management, interactions, natural hazards \\ Supplementary material for this articleis available online
}

\section{Abstract}

Fuvial floods aretypically investigated as 'events' at thesingle basin-scale, hence flood management authorities may underestimate thethreat of flooding across multiple basins driven by large-scale and nearly concurrent atmospheric event(s). We pilot a national-scale statistical analysis of the spatio-temporal characteristics of extreme multi-basin flooding (MBF) episodes, using peak river flow data for 260 basins in Great Britain (1975-2014), a sentinel region for storms impacting northwest and central Europe. During themost widespread MBF episode, 108 basins ( $46 \%$ of the study area) recorded annual maximum (AMAX) discharge within a 16 day window. Such episodesare associated with persistent cyclonic and westerly atmospheric circulations, atmospheric rivers, and precipitation falling onto previousy saturated ground, leading to hydrological response times $<40 \mathrm{~h}$ and documented flood impacts. Furthermore, peak flows tend to occur after 0-13 days of very severe gales causing combined and spatially-distributed, yet differentially time-lagged, wind and flood damages. These findings have implications for emergency responders, insurers and contingency plannersworldwide.

\section{Introduction}

Foods endanger lives, damage the built environment, causedisruption and accruesignificant economic losses. The Sendai Framework for Disaster Risk Reduction [1] recommendsbetter mapping and management in areasproneto flooding to increase resiliencethrough public and private investment in disaster risk prevention and reduction measures. The UK Climate Change Risk Assessment [2] highlighted that flood risks are already significant in the UK and are expected to rise as a consequence of climate change. Pragmatic and well-targeted actions were called for with respect to high magnitudeflood risksfor communities, businesses and infrastructures [2]. Anecdotally, high-magnitude flood episodes also tend to impact large areas covering multiple river basins [3-6].

To date, fluvial flooding has tended to be studied on abasin-by-basin basis with respect to physical processesand impacts [7-15]. Somestatistical methodsfor creating design floods rely on pooled datafrom multiplebasins [16, 17], but theseapproachesareindifferent to any spatial and temporal relationships in the data whereas multivariate extreme value statisticsare useful for estimating return periods for major events [18-20] and for characterizing spatially varying and time lagged extreme flows [21-23]. Within the reinsurance sector, weather-driven multi-basin 'catastrophe models' are widely used to estimate economic losses due to flooding [24, 25]. Statistical approachesto joint probabilities [21, 22, 26-29] have been extended to multi-basin flooding (MBF), as well as simulation of extreme flow events for northeast England using conditional proba bility models [30]. Historical MBF episodes have also been investigated in Germany [31, 32], and across Europe climate models have ben used to project economic losses [e.g. 33]. However, so far in Great Britain (GB) and elsewhere, there have been no national-scale analyses using simple and pragmatic statistics that specifically focus on the spatio-temporal 
characteristics of extreme MBF and their links with extratropical cyclones (ETCs), that areknown to affect themost extreme single basin floods [34].

The MBF approach here proposed overcomes the limitations of single-basin return period estimation, with the possibility of developing a national-scale return period for improved risk communication. A MBF episode can simultaneously impact very large regions, with the chance to overwhelm emergency responses, e.g. coordinated by the UK Environment Agency. In addition, MBF may coincide with ETCs, which together create a multi-peril scenario of floodwind impacts. Such episodes may be more severe than what planned for; illustratively, combined flood-wind impactsat the 16 year return period areincreased by the link between perils, costing an additional $£ 0.3$ billion for domestic UK properties [35].

We present a pragmatic approach for detecting and quantifying the characteristics of extreme MBF episodes and their linkswith ETCs. We use GB as pilot area, but deploy techniquesthat areapplicablewherever thereare gauged river flow data. We searched a window of 1 to 19 days for coincident peak flow annual maxima (AMAX) in 260 non-nested river basins during the 1975-2014period. Following sectionsdescribethedata, methodological approach and metrics, then the six most extensive and temporally distinct MBF episodes identified. We confirm that these most extensive MBF had widespread impacts [36-42] and mostly occurred during winter. A particularly powerful aspect of our approach isthat it iscompatiblewith thesynoptic-scale (i.e. $1000 \mathrm{~km}$ horizontal length scale) of atmospheric conditions and land-surface properties. This allows severe MBF episodes to be evaluated alongside cate gories of atmospheric circulation (Lamb weather types, LWTs), antecedent rainfall as a proxy for soil moisture (Standardized Precipitation Index, SPI), atmospheric rivers(ARs), and storminess(very severegale, VSG, frequency). Moreover, thehydrological response (joining time, $\boldsymbol{l}^{t}$ ) for largeand small basinsisexamined to determine lagged responses in thesystem. Finally, the causes and implications of extremeMBFand their relationship with ETCsare discussed.

\section{Peak flow data}

Highest instantaneous (15 min) peak flows $\left(\mathrm{m}^{3} \mathrm{~s}^{-1}\right)$ in each water year (1st October-30th September) were extracted from the 1975-2014 record. These annual maxima (AMAX) series were drawn from 260 gauged basins widely distributed across Great Britain (GB), within a 40 year block that provides the best compromise between spatial and temporal coverage. Our network of stations is non-nested (i.e. one gauge per basin) and covers $60.1 \%$ of GB land area (figure 1). This is equivalent to Network A used in a previous related study [43] but with more representative coverage across GB. The mean basin area $(A)$ is $484 \mathrm{~km}^{2}$, ranging from $12 \mathrm{~km}^{2}$ (Pointon Lode) to $9948 \mathrm{~km}^{2}$ (Thames), and average basin elevation is $149 \mathrm{~m}$ as.l. Data were obtained from the National River Fow Archiveusing WINFAPFEH v4.1: http://nrfaceh.ac.uk/content/winfap-fehfiles-version-history and, for Scotland, from the Scottish Environment Protection Agency.

\section{Methods}

A pragmatic metric that defines the hydrological severity of a multi-basin flooding (MBF) episode, particularly one that highlights the spatial distribution of basins involved, is not yet available. So far, the severity of a single-basin fluvial flow is readily defined by the peak discharge, and it is also possible to rank MBF severity using the most extreme peak flow of the basins under study [23, 29]. Alternatively, severity may be defined in terms of economic impact [33,35], but complete and comparable residential and commercial loss estimates are extremely difficult to obtain for all but the most severe historical events. Recent studies have begun to assess the severity of flooding episodes by considering the whole UK, effectively extending the paradigm applied to single-basin floods by looking at monthly mean river flows [3,12] and seasonal river flow accumulations [4].

The MBF metrics proposed here are based on a deliberately straightforward procedure that counts the total number of basins involved in each episode. Our principal metric, denoted $n_{\mathrm{g}}$, usesthesummed number of independent gauges that report a peak flow annual maximum (AMAX) within agiven multi-day timewindow $(L)$. This extends a previous single-day approach [43] to include MBF episodes where AMAX fall within a window of length of $L$ days from 1 up to 19 , ending on the day where most gauges report their AMAX, denoted $d_{\max }$.

The following procedure was implemented ( $R$ code in supplementary data available at stacks.iop.org/ ERL/12/114009/mmedia) to identify MBF and determinetheir $n_{\mathrm{g}}$. Firstly, for each day $j$ determine $n_{\mathrm{g} . \mathrm{j}}$ and list these in descending order, creating the list of MBF episodes for $L=1$ day. Then for each $L>1$, using episodes of the $L=1$ day list anew for each $L$, follow these 4 steps: (1) ascertain that the current episode (C) is the largest (i.e. greatest 1 day $n_{\mathrm{g}}$ ) as yet unamalgamated remaining on the list; (2) identify any other basins reaching their AMAX within the specified timewindow before $C$, (3) add all their $n_{\mathrm{g}, \mathrm{j}}$ to $C$ scount and flagthesmaller episodesasbeing amal gamated with $C$, which prevents any day contributing to more than 1 episode for a given $L$; (4) repeat (1)- (3) until no more amalgamation is possible. Hence, when considering the $n_{\mathrm{g}}$ metric, the most extreme MBF episode is defined as that with the greatest number of basins exhibiting near concurrent AMAX within the specified time window $(L)$. However, two other characteristics 


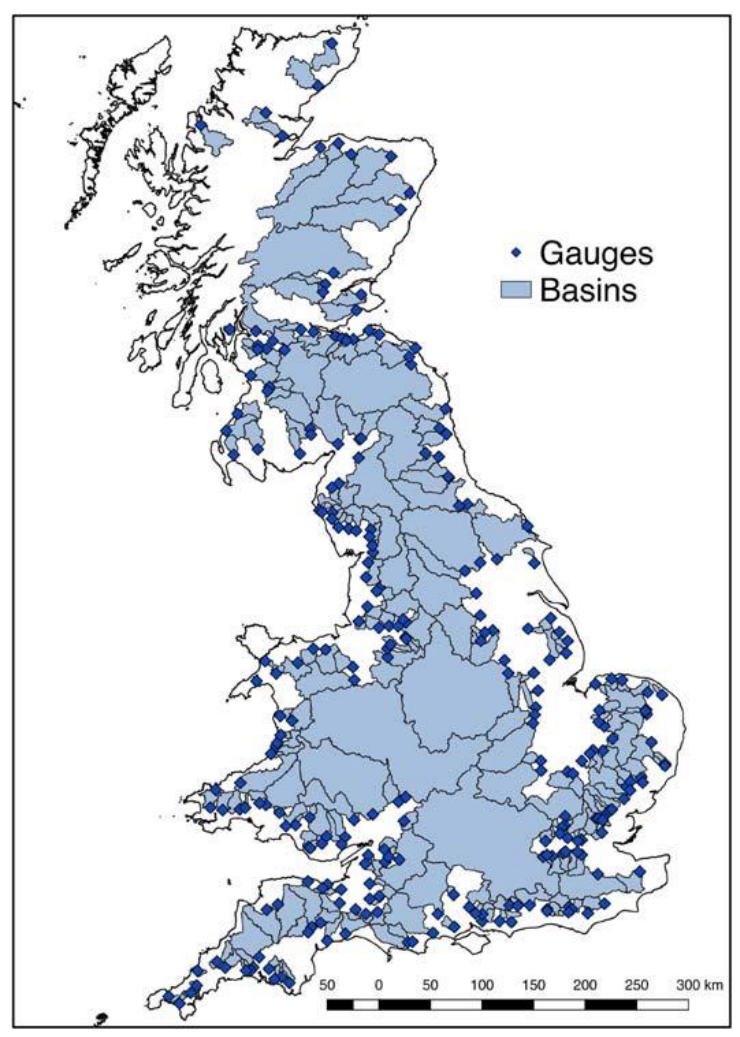

Figure 1. Network of hydrological stations and related areas of basins used in theanalyses. The 260 non-nested gauges were selected from an initial network of 648 (figure S1) based on record length, and geographic coverage. Some areas are under-represented (e.g. east England) because they are either ungauged or do not have datafor the 1975-2014 period.

were derived for each episode. These are: (i) themultibasin Flood Yield ( $m F Y$, supplementary data $\mathrm{A})$; and (ii) the total drained area (TDA) of the basins reaching their AMAX within an episode. These usethe same list of episodes, and basins, defined by the $n_{\mathrm{g}}$ metric, but an alternative quantity to rank severity. The $m F Y$ index is potentially biased towards small basins, whereas TDA intrinsically assigns greater weight to larger basins.

The AMAX dates for individual river basins are denoted event set $A$. Event set $B$ comprises extreme MBF episodes with severity defined in terms of $n_{\mathrm{g}}$, taking the largest temporally distinct episodes defined by six key time windows with different lengths, varying from 1 to 16 days (figure 2, table 1), and the 10 next largest episodes in each key time window. Event set $C$ contains the most extreme $L=13$ days MBF episode for each water year defined using $m F Y$, and set $\mathrm{D}$ is similar except defined by TDA. Event set $\mathrm{E}$ consists of the six most extreme episodes defined by $n_{\mathrm{g}}$ (figures 2, 3(a) and (b), table 1). Replicated days are removed such that days occurring in two or more window lengths' episodes, necessary only in B and
E, are never counted twice. Smilarly, days with $>1$ single-basin AMAX are not counted repeatedly for national-scale analyses (figures $3(c)$ and $(d)$ ). Where different observations need to be shown basin-bybasin, multiple basins recording their AMAX are permitted to contribute on the same day (figure 4).

\section{Results}

4.1. Characterizing severe multi-basin flooding (MBF) episodes

The most extreme multi-basin flooding (MBF) episodes defined by $n_{\mathrm{g}}$, i.e. by the concurrent number of basins reaching their peak flow annual maxima (AMAX), obtained from 19 time window lengths $(L)$, comprise fivetemporally distinct episodes (event set $E$, figure 2 and table 1). These are: $d_{\max }=27 / 12 / 1979$ (66 basinsinvolved, $18.6 \%$ of study area, window length $L=$ 1 day); 30/10/2000 (68, 14.1\%, $L=2$ days); 01/01/2003 (75, 24.9\%, $L=4$ days); 02/12/1992 (96, 22\%, $L=$ 8 days); and 01/02/1995 (108, 46.5\%, $L=16$ days), with $d_{\max }$ representing the day, in each episode, where 

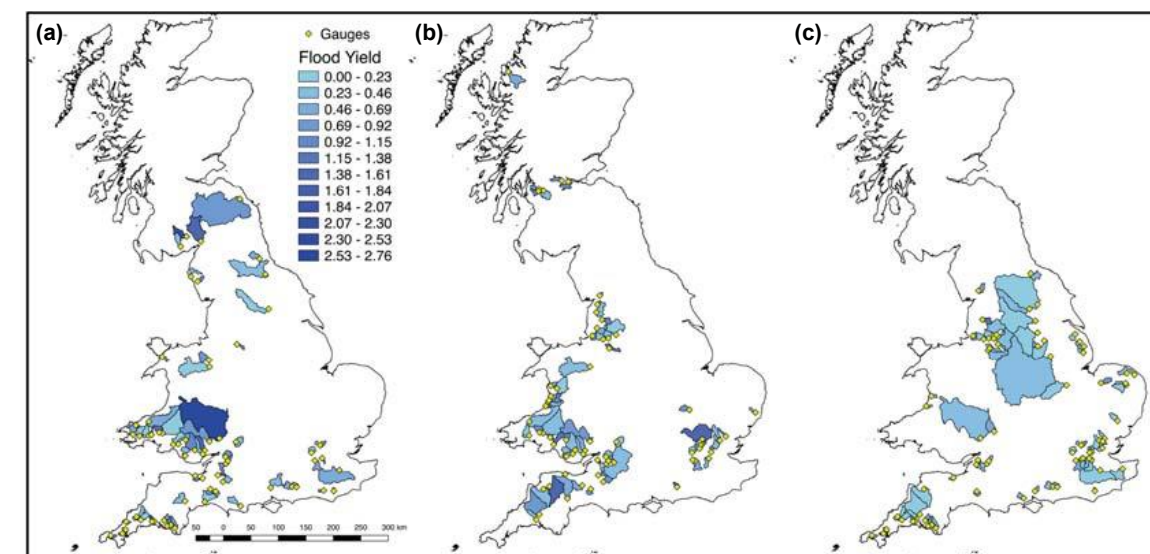

(d)
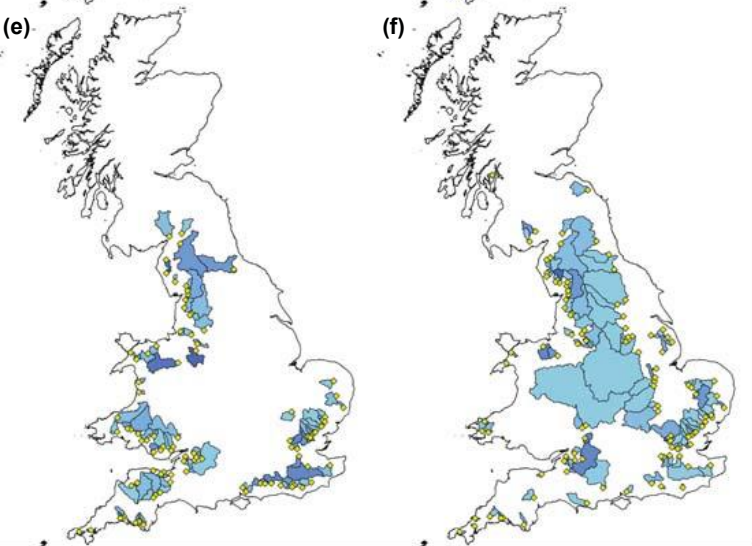

Figure 2. Distribution of basins contributing to the extreme multi-basin flooding (MBF) episodes in Great Britain (GB) during 1975-2014 for six timewindow lengths ( $L$, event set $E$ ). Themaps show respectively: (a) $L=1$ day $\left(d_{\max }=27 / 12 / 1979\right) ;(b) L=2$ days $\left(d_{\max }=30 / 10 / 2000\right) ;(d) L=4$ days $\left(d_{\max }=01 / 01 / 2003\right) ;(d) L=6$ days $\left(d_{\max }=30 / 10 / 2000\right) ;(e) L=8$ days $\left(d_{\max }=02 / 12 / 1992\right) ;$ and $(f) L=16$ days $\left(d_{\max }=01 / 02 / 1995\right)$. Food Yield $(F Y)$ is a severity metric that represents each basin's peak flow annual maximum (AMAX) normalized by the relative basin area $(A)$ and $d_{\max }$ is defined as the day where the largest number of AMAX have been registered within each episode.

the largest number of AMAX have been recorded. If different time windows return the same date, the window with the largest number of concurrent $A M A X$ is given. However, the $L=6$ days episode $(30 / 10 / 2000$, figure 2( $d$ ), table 1) is included because the number of basins involved (86) and total drained area (TDA, 24 $971 \mathrm{~km}^{2}$ ) are both much larger than the $L=2$ days episode. Figure 2 shows the regional distribution and basin-by-basin Flood Yield ( $F Y$, supplementary dataA) severity of these six episodes.

The $n_{\mathrm{g}}$ metric ranges from 66 ( $L=1$ day) to 108 ( $L=16$ days), plateauing at $L \cong 13$ days (figure $3($ a) ). For all timewindows, the number of co-occurrences is notably larger than expected by chance $(p<0.01$, binomial test, supplementary data F.1). The TDA ranges from $17787 \mathrm{~km}^{2}$ ( $L=2$ days) to $58491 \mathrm{~km}^{2}$ ( $L$ $=16$ days), again plateauing at $L \cong 13$ days (figure $3(b))$. These areas correspond to a TDA percentage of $14.1 \%$ and $46.5 \%$ of the area of the 260 gauged basins respectively, or $8.5 \%$ and $27.9 \%$ of thetotal land area of Great Britain (GB, figure 3(b), table 1). Window length $L=13$ days is used to define event sets $C$ and $D$ as it captures thelargest episodes whilst retaining the maximum temporal resolution.

Figure $3(d)$ shows that the six most extensive MBF episodes(event set E) tended to occur during thewinter (December-February), closely matching the pattern of event sets A-D. However, AMAX occurrences in January are more common for MBF episodes (event sets B-E) than for single-basin events (event set $A$ ). Spatially, event set E episodes impacted a substantial proportion of our study basins (figures 2 and 4(d)). However, when considering more episodes (event sets B-D) the spatial distribution of basins impacted is even larger, with all the study area affected (figures $4(a),(b)$ and (c)). Figure 4 shows also that the relative frequency of AMAX occurrences is homogenously distributed across all the basins for 
Table 1. Extreme multi-basin flooding (MBF) episodes in Great Britain (GB) during 1975-2014 (event set E). Observations are derived from 19 timewindows up to 18 days prior to $d_{\max }$ (i.e. the day wherethelargest number of peak flow AMAX have been registered). See main text for details (a) Window length $(L)$ in days; (b) Total drained area $\left(T D A, \mathrm{~km}^{2}\right)$ involved in each episode (i.e. sum of theareas of all involved basins) : (c) TDA percentage (\%) of the 260 basins affected within each episode; (d) Percentage (\%) of GB land area affected within each episode; (e) Dates of episodes, wherethetop row is $d_{\text {max }}$; (f) Number of basinswith AMAX registered within each distinct day; (g) Total
number of basinswith AMAX registered within each distinct episode; (h) Percentage (\%) of total number of basins (out of 260) with number of basinswith AMAX registered within each distinct episode; (h) Percentage (\%) of total number of basins (out of 260) with
concurrent AMAX per episode; (i) Daily Lamb weather type (LWT); (j) Average joining time ( $\boldsymbol{t}$, in days), within an episode, for larger basins concurrent AMAX per episode; (i) Daily Lamb weather type (LWT); (j) Average joining time ( $l$, in days), within
$\left(A \geq 1000 \mathrm{~km}^{2}\right)$; (k) Average $t$ for small basins $\left(A<1000 \mathrm{~km}^{2}\right)$. In (j) and $(\mathrm{k})$ uncertainties are 1 standard error.

\begin{tabular}{|c|c|c|c|c|c|c|c|c|c|c|}
\hline $\begin{array}{l}\text { (a) } \\
\text { Timewindow } \\
\text { length }(L, \\
\text { days })\end{array}$ & $\begin{array}{c}\text { (b) } \\
\text { Total } \\
\text { drained } \\
\text { area } \\
(T D A, \\
\left.\mathrm{km}^{2}\right)\end{array}$ & $\begin{array}{c}\text { (c) } \\
\text { Total } \\
\text { drained } \\
\text { area } \\
(T D A, \\
\%)\end{array}$ & $\begin{array}{c}\text { (d) } \\
\mathrm{GB} \\
\text { area\% }\end{array}$ & $\begin{array}{l}\text { (e) } \\
\text { Date }\end{array}$ & $\begin{array}{c}(f) \\
\text { No. } \\
\text { basins } \\
\text { per day }\end{array}$ & $\begin{array}{c}(\mathrm{g}) \\
\text { No. } \\
\text { basins } \\
\text { per } \\
\text { episode }\end{array}$ & $\begin{array}{c}\text { (h) } \\
\text { No. } \\
\text { basins } \\
\%\end{array}$ & $\begin{array}{l}\text { (i) } \\
\text { LWT }\end{array}$ & $\begin{array}{c}(\mathrm{j}) \\
\text { Average } \\
\text { joining } \\
\text { time }(A \geq \\
\left.1000 \mathrm{~km}^{2}\right)\end{array}$ & $\begin{array}{c}(\mathrm{k}) \\
\text { Average } \\
\text { joining } \\
\text { time }(A< \\
\left.1000 \mathrm{~km}^{2}\right)\end{array}$ \\
\hline 1 & 23399 & 18.6 & 11.18 & 27/12/1979 & 66 & 66 & 25.3 & C & - & - \\
\hline 2 & 17787 & 14.1 & 8.50 & $\begin{array}{l}30 / 10 / 2000 \\
29 / 10 / 2000\end{array}$ & $\begin{array}{c}62 \\
6\end{array}$ & 68 & 26 & $\begin{array}{c}\mathrm{C} \\
\mathrm{CSW}\end{array}$ & 2 & $1.91 \pm 0.0$ \\
\hline $\begin{array}{l}4(3 \& 5 \\
\text { same } d_{\max } \\
\text { as } 4)\end{array}$ & 31370 & 24.9 & 14.99 & $\begin{array}{l}01 / 01 / 2003 \\
31 / 12 / 2002 \\
30 / 12 / 2002 \\
29 / 12 / 2002\end{array}$ & $\begin{array}{c}34 \\
7 \\
29 \\
5\end{array}$ & 75 & 28.7 & $\begin{array}{l}\text { C } \\
\text { S } \\
\text { C } \\
\text { C }\end{array}$ & $2.83 \pm 0.3$ & $2.94 \pm 0.1$ \\
\hline 6 & 24971 & 19.8 & 11.93 & $\begin{array}{l}30 / 10 / 2000 \\
29 / 10 / 2000 \\
28 / 10 / 2000 \\
27 / 10 / 2000 \\
26 / 10 / 2000 \\
25 / 10 / 2000\end{array}$ & $\begin{array}{c}62 \\
6 \\
0 \\
2 \\
0 \\
16\end{array}$ & 86 & 33 & $\begin{array}{c}C \\
\text { CSW } \\
C \\
W \\
W \\
\text { NW }\end{array}$ & $5 \pm 1$ & $4.93 \pm 0.2$ \\
\hline $\begin{array}{l}8 \text { (7 same } \\
\left.d_{\max } \text { as } 8\right)\end{array}$ & 27674 & 22.0 & 13.22 & $\begin{array}{l}02 / 12 / 1992 \\
01 / 12 / 1992 \\
30 / 11 / 1992 \\
29 / 11 / 1992 \\
28 / 11 / 1992 \\
27 / 11 / 1992 \\
26 / 11 / 1992 \\
25 / 11 / 1992\end{array}$ & $\begin{array}{c}49 \\
1 \\
19 \\
2 \\
0 \\
5 \\
17 \\
3\end{array}$ & 96 & 36.8 & $\begin{array}{c}\text { C } \\
\text { SW } \\
\text { SW } \\
\text { S } \\
\text { ANE } \\
\text { SW } \\
\text { W } \\
\text { SW }\end{array}$ & $7 \pm 0.8$ & $5.91 \pm 0.3$ \\
\hline $\begin{array}{l}16(9 \text { to } 15 \\
\& 17 \text { to } 19 \\
\text { same } d_{\max } \\
\text { as } 16)\end{array}$ & 58491 & 46.9 & 27.94 & $\begin{array}{l}01 / 02 / 1995 \\
31 / 01 / 1995 \\
30 / 01 / 1995 \\
29 / 01 / 1995 \\
28 / 01 / 1995 \\
27 / 01 / 1995 \\
26 / 01 / 1995 \\
25 / 01 / 1995 \\
24 / 01 / 1995 \\
23 / 01 / 1995 \\
22 / 01 / 1995 \\
21 / 01 / 1995 \\
20 / 01 / 1995 \\
19 / 01 / 1995 \\
18 / 01 / 1995 \\
17 / 01 / 1995\end{array}$ & $\begin{array}{c}19 \\
16 \\
9 \\
9 \\
10 \\
16 \\
14 \\
3 \\
2 \\
3 \\
3 \\
1 \\
2 \\
0 \\
0 \\
1\end{array}$ & 108 & 41.4 & $\begin{array}{c}\text { W } \\
\text { SW } \\
\text { ANE } \\
\text { C } \\
\text { C } \\
\text { S } \\
\text { N } \\
\text { C } \\
\text { W } \\
\text { CNW } \\
\text { C } \\
\text { C } \\
\text { C } \\
\text { CS } \\
\text { SW } \\
\text { CS }\end{array}$ & $13.73 \pm 0.9$ & $11.97 \pm 0.3$ \\
\hline
\end{tabular}

all event sets considered, possibly excluding Scotland for set E. This contrasts with precipitation distributions during winters dominated by westerly or cyclonic patterns [44], when rainfall tends to respectively decrease from west-to-east or is heavier in the east.

The average joining time ( $\boldsymbol{d}$, supplementary data B) for larger $\left(A \geq 1000 \mathrm{~km}^{2}\right)$ and smaller $(A<1000$ $\mathrm{km}^{2}$ ) basinswithin MBF episodeswascompared. Considering time windows $(L)$ separately for event set $E$ (figure $S 3$ ), only when $L=2$ or 16 days do larger basins join significantly later than small ones ( $t$ test non-paired, supplementary data F.2), and the delays were modest, just 0.1 and 1.8 days respectively (table 1). Event sets B-D replicate this, showing occa sional significance but a difference in $t<48 \mathrm{~h}$. A time to peak $\left(T_{\mathrm{p}}\right)$ response analysis (supplementary dataC) $[45,46]$ for larger basins further indicates $T_{p}<40 \mathrm{~h}$, again less than the $\sim 13$ day time-span that appears to define extreme MBF episodes

\subsection{Relationship to inundation episodes}

Severity measured by $n_{\mathrm{g}}$ is a proxy for overbank flow and fluvial flood extent. Only a fraction of the basins' areas will actually be inundated. However, the six extremeMBFepisodes(event set E, figure2) all resulted in widespread flooding demonstrating the relevance of the $n_{\mathrm{g}}$ metric as a diagnostic: 

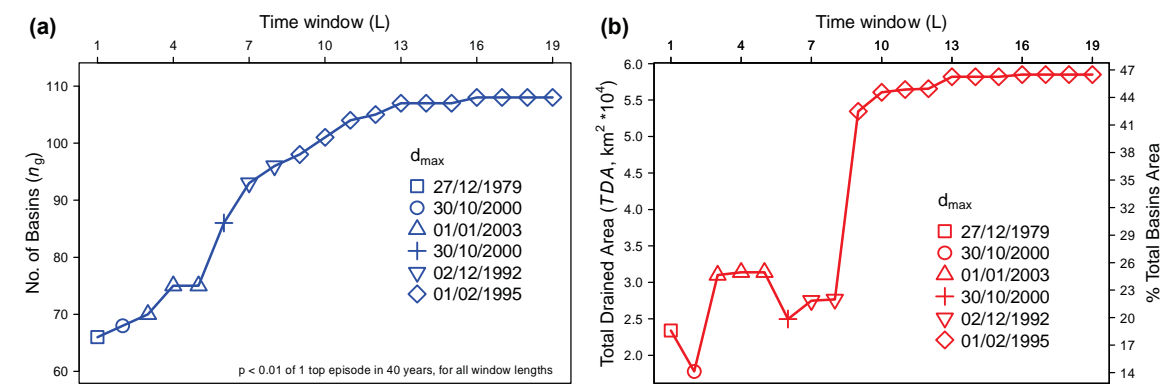

(c)

(d)
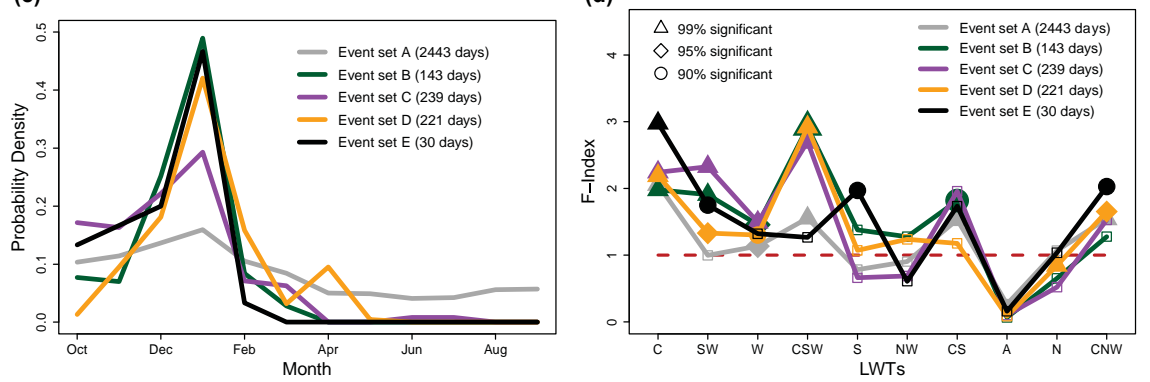

Figure 3. Characteristics of the extreme MBF episodes (event set E), compared to event sets A, B, C and D. (a) Maximum number of basins with concurrent AMAX $\left(n_{\mathrm{g}}\right)$ versus window length $(L)$, defining the most extreme episodes (event set $\left.\mathrm{E}\right) ;(b)$ as in $(a)$ but for total drained area $(T D A)$, measured by $\mathrm{km}^{2}$ and by percentage (\%) of total study area; ( $C$ temporal distribution of peak flow AMAX occurrences for the extreme episodes in event sets A-E; and (d) frequency of Lamb Weather Types (LWTs) associated with event sets A-E with respect to their expected occurrence, calculated as a flood index (F-Index) [43]. Duplicated days in event setsin (c) and (d) have been removed. Sgnificance was determined using the binomial test (supplementary data F.1 and F.3). The LWTs shown are based on event set E; event sets A-D also contain other LWTs (figure S2).

- The December 1979 episode (figure 2(a)) was the most severe in South Wales since 1960 and in some areastheworst in acentury, causing extensivefloods that killed four people, necessitated theevacuation of hundreds and caused millions of pounds of damage [36].

- The Autumn 2000 episodes (figures 2(b) and (d)) were described as the most devastating in England since 1947, and associated with thewettest 12 month period since $1776[37,38]$.

- The January 2003 episode (figure 2(d) was reported by the Environment Agency in FoodLink [39] with most severe floods in the East Midlands, where the Trent basin had 118 flood warnings and 14 flood watchesissued between 29/12/2002 and 03/01/2003.

- TheNovember/December 1992episode(figure2(e)) was reported by the UK Met Office [40] after floods impacted southern England during the night of 25th/26th November. However, the worst phase occurred on the29th, when flooding in Wal es devastated homesand caused widespread road and rail way disuptions.

- The February 1995 episode (figure 2(ff) caused severe floods on at least 7 rivers, following heavy frontal precipitation in January 1995 which was $79 \%$ above the 1961-1990 average [41, 42].
4.3. Relationship to atmospheric patterns

Daily UK synoptic-scal eatmospheric patternsarecharacterized by Lamb weather types (LWTs) [47, 48]. The frequency of LWTs for days during extreme singleand multi-basin peak flow episodes was compared with the entire 40 year catalogue of LWTs (figure $3(d)$ ). In this comparison, a flood index (F-Index, supplementary data D) [43] is defined as the ratio of observed to expected frequency of LWTs. This was undertaken for event sets: A (2443 days), B (143 days), $C$ ( 239 days), $D$ ( 221 days) and $E$ ( 30 days), excluding replicate dates. Statistical significance of the F-Index was calculated using a binomial test (supplementary dataF.3).

Overall, thecyclonic (C-type) LWT isstrongly associated with the peak flows with a $99 \%$ statistically significant F-Index $\geq 1.98$ for all event sets considered, in particular flooding was $\sim 3$ times more likely than expected during C-type occurrences for event set $\mathrm{E}$. The south-westerly (SW), westerly (W), and cyclonic SW (CSW) typesarealso associated with AMAX events $(p<0.01,0.05$ and 0.1$)$, and therefore more likely linked with widespread flooding. Southerly (S) types are significantly represented in event sets $E$, but not in event sets A-D (figure 3(d)). Therefore, a pattern of Cand $\mathrm{W}$-types contributing to widespread peak flows is depicted and the multi-basin event sets B-E show very 

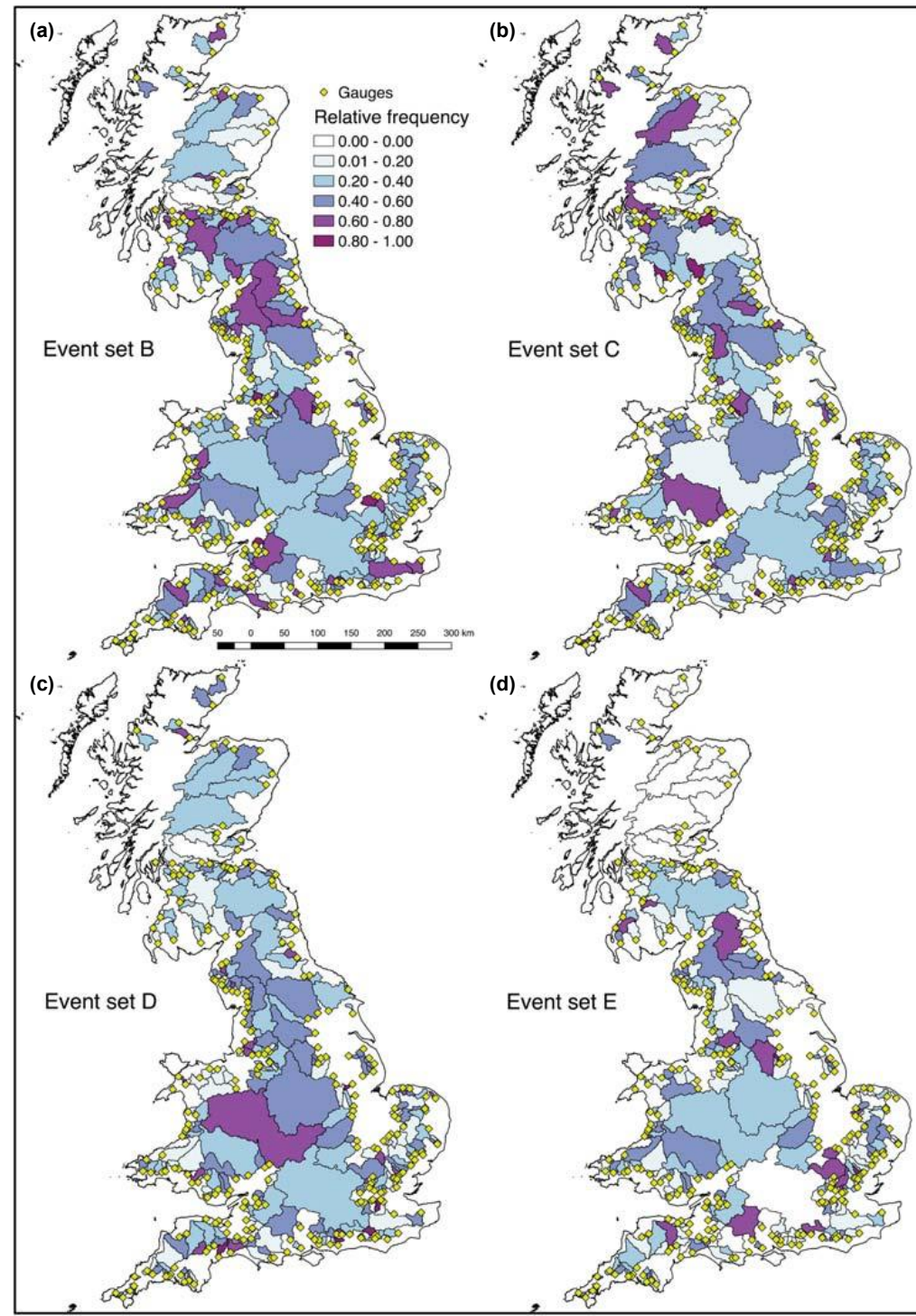

(d).

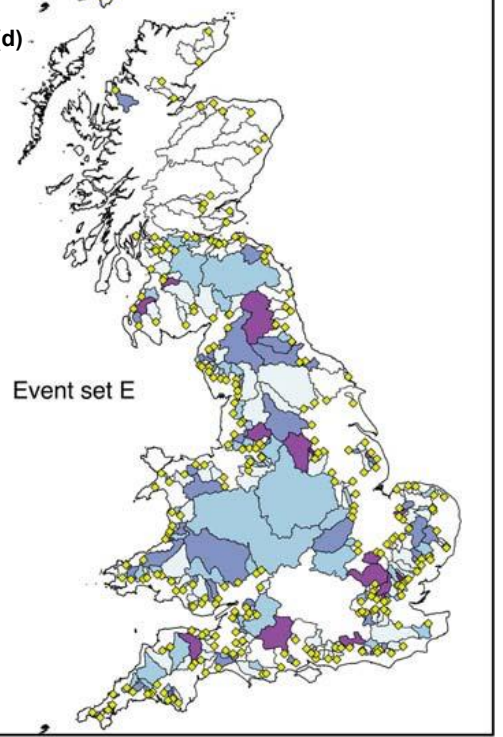

Figure 4. Distribution and relative frequency of occurrence of peek flow annual maxima (AMAX) within event setsB, C, D and E. (a) Event set B; (b) event set C; ( $d$ event set $D$; and (d) event set $E$. Thecolour scal e is a ratio (i.e. from 0 to 1 ) of AMAX occurrencesin a given basin relative to the basin with thelargest number in that panel, with dark colours indicating most occurrences

similar F-Index resultswhen compared to single-basin AMAX (event set A, figure S4).

It is also of interest if these circulation systems are particularly 'wet'. Atmospheric rivers (ARs) are corridors of intense horizontal water vapour transport within the warm conveyor belt of extratropical cyclones (ETCs) $[34,49]$. The dates of event set $E$ episodesarecompared with theBrands $\&$ al ARarchive [50] derived from ERA-Interim reanalysis [51]. Four out of the fivetemporally distinct MBF episodes' most extreme flows (i.e. $d_{\max }$ dates) occurred on the same day as an AR, which on average happen on only $30 \%$ of extended (October-March) winter days $(p<0.01$, binomial test, supplementary dataF.4).

4.4. Relationship with antecedent soil moistureconditions

Wet soil moisture antecedent conditions increases the likelihood of flooding [52]. The standardized precipitation index (SPI, supplementary data E) $[53,54]$ is widely used as a proxy for this physical property and 3-24 month SPI values are distinctively high for historical flooding episodes [55-57]. Whilst the sample of 


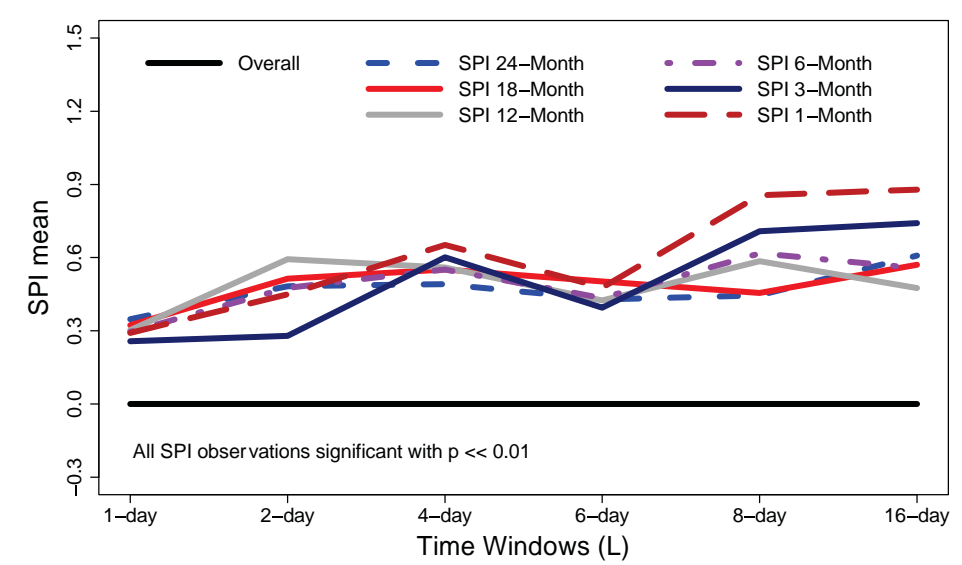

Figure 5. Mean standardized precipitation index (SPI) for episodes within event set B for each window length $(L)$ and SPI timescale (24-1 Month). Lines are episodes' SPI averages with coloursindicating: dashed blue = SPI 24 Month; red = SPI 18 Month; grey = SPI 12 Month; dashed purple = SPI 6 Month; dark blue = SPI 3Month; dashed brown = SPI 1 Month. Theblack linerepresents theoverall (40 year, 1975-2014) SPI average i.e. zero by definition. All episodes have SPI that are significantly different from thelong-term mean at $99 \%$ level ( $t$-test, not paired, supplementary data F.5).

episodesin event set Eistoo small to show apattern, SPI aggregated across impacted basins [58] is higher than average across all window lengths $(L)$ for event set $B$ ( $p \ll 0.01, t$-test non-paired, supplementary data F.5), increasing with $L$ (figure 5 ). Event set $\mathrm{C}$, based on the multi-basin flood yield ( $\mathrm{mFY}$ ) metric, by incorporating aforced regularized annual sampling, demonstrates that flood magnitudeisgreater in 'wet' spells $(S P I>0.5)$ than 'dry' periods $(\mathrm{SPI}<-0.5)$ with mean $m F Y=$ $26.9 \pm 3.4(1 \sigma)$ and $17.1 \pm 1.3(1 \sigma)$ as calculated from SPI 12 Month. Indeed, for this comparison, all except SPI 1 Month are significant ( $p<0.05$, $t$-test, 2-tailed). Event set $\mathrm{D}$ (based on TDA) shows no signal for this well-established flood-SPI connection, suggesting that the metric based on $m F Y$ might better reflect physical processes.

\subsection{Relationship to very severe gales}

Flooding and severe wind have been reported for some ETCs impacting western Europe [3, 59]. A potential association between extreme MBF and severe storms was, therefore, investigated. In a year-by-year analysis the most extreme $L=13$ days $m F Y$ episodes (event set C) correlates positively with the number of days with very severe gales (VSG) as defined by the Jenkinson Gale Index [48] in that year $(r=0.41, p=0.0088$, 2tailed $t$-test, supplementary dataF.6) (figure6). Taking the most severe $50 \%$ and $30 \%$ of years for wind and flow respectively, co-occurrence is expected 6.0 times in 40 years, but 10 are observed ( $p=0.021$; Monte Carlo simulation with $n=10000$ ), making coincidence of extremes $67 \%$ morelikely than what would be expected by chance.

Furthermore, the timing of these episodes is the basisfor insightsinto thephysical processesat work. For 5 out of 10 observed co-occurrences, themost extreme peak flows recorded on $d_{\max }$ are on a day with VSG, and 9 of 10 peak flows arewithin 0-13 days after aVSG day $(p \ll 0.001$, binomial test). This contrasts with 0 out of 10 peak flow episodes found in the preceding 0 13 days of a VSG day. In agrement with the flood-SPI analysis, therelationship isnotably lessstrong for event set $\mathrm{D}$ (based on $T D A$ ), indicating that $m F Y$ may better reflect physical processes in storm systems.

Wet ground is a pre requisite to the most severe peak flow episodes, but there is also a link with gales. Sx out of the 10 most severe episodes have a SPI 12 month between + 0.4 and + 1.1 (figure 6 , whitecircles), whereas less severe episodes tend to show a negative SPI (figure6, black circles). The two outliersin figure6 (1983 and 2014) reflect previous studies [4, 44, 60-63] that showed that the number of cycloneswere particularly high over the GB during these years. However, the largest $m F Y$ for these two episodes may be depressed by the AMAX measure of extremeness which, by definition, limits the number of occurrences per year. Therefore, these observations are likely valid given, if influenced by the analytical method used.

\section{Disqussion}

\subsection{A new multi-basin approach}

We have presented various diagnostics for the evaluation of multi-basin flooding (MBF) episodes. The first metric $\left(n_{\mathrm{g}}\right)$ detects key 'episodes' by summing the concurrent number of basins attaining their peak flow annual maximum (AMAX) within a given time window $(L)$, then ranking the episodes based on $n_{\mathrm{g}}$. We also considered episodes ranked by total drained area $(T D A)$ and multi-basin flood yield $(m F Y)$. When episodesareidentified in terms of $n_{\mathrm{g}}$, thisgivesperhaps 


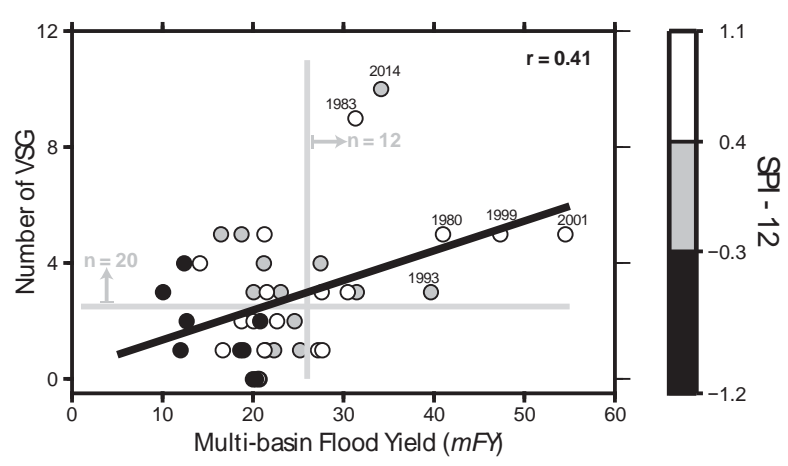

Figure 6. Number of very severe gales (VSG) versus extreme multi-basin Flood Yield ( $m F Y$ ) episodes for each water year (1975-2014, event set C). Black circles = SPI 12 month $<-0.3$; Grey circles $=-0.3<$ SPI 12 month $<0.4$; Whitecircles $=$ SPI 12 month $>0.4$. $n$ $=20$ represents the $50 \%$ most extreme VSG and $n=12$ the $30 \%$ most extreme $m F Y$ episodes.

undue weight to small basins, but TDA emphasizes larger rivers. The $m F Y$ can either weight small basins, when calculated as here or large ones if area and flow were each summed before dividing them. All are practical options, but awareness of any biases and use of multiple metrics is recommended to ensure robust insights.

There are various advantages with this approach to MBF analysis. First, because of the different time windows $(L)$ used within each metric, it enables the identification of extreme peak flow episodes that are driven by persistent rain-bearing weather systems by accounting for variations in timelags between pre cipitation and peak flows, that depend on rainfall properties, basin areaand geology. Second, it providesa national-scale flood measure allowing more meaningful comparison with synoptic-scale weather patterns than at the scale of individual basins, regardless of the catchment area[35]. Third, whichever metricisselected areturn period that is applicableacrossawholecountry can be estimated.

A single, national rather than basin-scale, return period has a potentially important role in risk communication. Such metrics could address the question often posed by flood managers: "Why is there a 1 in 100 year flood event every year? This impression arises because return period estimates are traditionally based on flows at a single gauge. The MBF metrics proposed here would yield the 1-in-100 year episode based on a return period estimate that integrates information across all basins in anetwork.

5.2. Widespread concurrent impacts

Our resultsshow that extremeMBFepisodesaffect large areas (figure 2), with likely commensurate damages [36-42]. For instance, the $L=16$ days episodecaptures $\sim 46 \%$ of thestudy area, or $\sim 27 \%$ of Great Britain (GB), with 108 basins concurrently reaching their AMAX (figures2 ( $f$ ), 3(a) and (b), table1). Aspects of thephysical processes driving these widespread episodes appear similar to those deduced from single-basin studies $[3,4,7,8,11-13,15,44]$. First, W- and C- Lamb weather types (LWTs) associated with MBF (figure 3(d), table 1) have been linked to frequent floods $[43,64]$, the wettest wintersin England and Wales [44], and $>80 \%$ of extremeflows on the River Eden (UK) [64]. TheWtype, in particular, represents one of the main drivers of high rainfall and flows in the UK $[64,65]$ as well as flooding throughout central Europe [e.g. 64]. Second, $\mathrm{MBF}$ islarger (by $\mathrm{mFY}$ ) in wet years, i.e. when the SPI $>0.5$, and in longer time windows when the SPI is higher, suggesting antecedent soil moisture conditions may play a role. Third, as for single basin floods [34], the most extreme MBF episodes coincide with atmospheric rivers (ARs).

The observation that singlebasin flooding in GB occursmostly during winter also appliesto MBF (figure 3(c). Thisisdueto frequent stormsand their associated precipitation [66], combined with lower evapotranspiration, and wetter antecedent soil conditions (figure 5) that ultimately combine to generate higher flows $[11,12]$. However, compared to singlebasin flooding, the largest MBF are even more strongly typified by occurrence in January (figure $3(d)$ ), when the most favourable atmospheric flood-generating conditions (C, SW, W and CSW circulation types) aremorelikely. This close association with synoptic weather is not surprising, but neither it is required by or self-evident $a$ priori from single basin analyses.

A key feature that distinguishes large MBF from their single-basin counterparts is their duration (i.e. $\cong 13$ days, figures $3(a)$ and $(b))$. This is greater than currently accounted for in other studies[28], and indicates that at least one notable source of persistence or 'memory' in the physical system is required. With a time to peak $\left(T_{\mathrm{p}}\right)<40 \mathrm{~h}[45,46]$, these sources cannot bewithin the channelized flow paths, a view supported empirically by larger basins joining episodes at essentially the same time as smaller ones (table 1, figure S3). This observation also rules out, from the possible sources of 'memory', reservoirs delaying flow outside 
of the channels, and is reconciled by the fact that concentration timeincreases with basin area [67]. Thus, we postulate that a 'memory' exists in either antecedent soil or groundwater levels [55-57] (figure 5) and/or in persistent atmospheric patterns during notably wet years $[3,4,12,44,59,61]$, such as known ETCs clustering in extreme winters [63]. These elements of 'memory' likely exist for larger European rivers (e.g. Rhine), although they are lesseasily decoupled because timescales attributable to the processes overlap more.

5.3. Compounding flood and wind impacts Extratropical cyclones (ETCS) were identified as a driver of MBF, firstly via an association with cyclonic LWTs and ARs. Also, when considering these high flows in terms of extreme $m F Y$ for each water year within $L=13$ days (event set $C$ ), a relationship with damaging winds predominantly caused by ETCs is also demonstrated. A significantly positive correlation exists between VSG and MBF, with co-occurrence of extremes $67 \%$ more likely than by chance and high flows occurring within 0-13 days after a VSG day. Hence, building on case studies of notable years $[4,12,13,44,61,62]$ and the Trent basin in central England [35], this is the first systematic, national-scale evidence that the severest aspects of wet and windy winterstend to co-occur and are linked by the physical processes associated with ETCs. Often these phenomena are viewed separately: severe ETCs bring extreme winds [68] whilst slower moving, less windy ETCs bring large accumulated rainfall totals and extensive flooding in GB [63, 66, 69]. Thus, our evidence of coincident widespreadflood and wind on the same day in 5 out of 10 years and within 13 days in another 4 of those years contradicts a prevailing view that storms such as Desmond was exceptional in bringing both very severe wind and widespread flooding $[3,59]$. These findings also highlight the importance of considering longer time-lags when assessing dependencies between weather-driven hazards where both may not occur in thesamedefined extremeepisode. As far we are aware, this is the first statistical evidence of a time-lagged link between widespread flooding and severe wind for any nation. Our methodology also enables potential detection of such inter-dependencies elsewhere.

One implication of coincident floods and severe winds is that worst-case years are likely more severe than previously thought. With the association apparently strongest for themost extremeepisodes, theeffect of this co-occurrence likely increases combined floodwind insurance losses for domestic UK properties in bad years [35]. Moreover, GB is located beneath the North Atlantic storm track and is, therefore, affected by the passage of ETCs [66] which bring extreme winds [68] that can subsequently affect central Europe $[63,70]$. Snce ETCs can continue to strengthen after landfall, this effect may extend to a much larger physical and financial scalethan theGB alone. Furthermore, there is a likely threway association between widespread flooding, severewind and storm surgesthat warrants investigation.

\subsection{Operational implications}

The Environment Agency is responsible for contingency planning, forecasting and managing the consequences of widespread flood episodes. Regional 'footprints' of past severe episodes (figure 2) reveal the extent to which authorities in neighbouring areas could be impacted simultaneously. This is relevant when coordinating and sharing equipment and personnel during such episodes. For instance, the Midlands region of theEnvironment Agency liesin apivotal loca tion since it may becalled upon to provideresourcesto affected areasto theNorth and South. During thesevere flooding in December 2015, personal and equipment were drawn from regions hundreds of kilometres away from the epicentre of Northwest England and Southern Scotland. This might not be feasible in the event of a MBF episode on the scale of January/February 1995 (figure 2(f $f)$. However, knowledge of the likelihood and pattern of MBF provides abasisfor role-play exercises as part of the contingency planning for such episodes.

Both the UK National Food Resilience Review [71] and UK Climate Change Risk Assessment [2] recognise interdependencies between critical networks (e.g. electricity, water and transport) and the need to manage indirect flood impacts on the economy. However, their emphasis remains on integrated, yet single-basin solutions involving 'natural' flood management, improved property- and asset-level resilience, and planning controls. Widespread flooding in Australia in 2011, and multiple events in Central Europe since 2002, show the need for a higher-level strategy for managing extensive, transboundary flooding [72]. Moreover, the likelihood of MBF could increase with ETCsintensity and ARsfrequency and magnitude expected to rise under anthropogenic climate change $[49,66,70,73-75]$.

\section{Acknowledgments}

The authors thank the Scottish Environment Protection Agency for providing Scottish peak flow data, the Centre for Ecology and Hydrology for the SPI data, Swen Brands for providing the AR array along with detailed information, the RCUK (CENTA NERC) for thefunding availability, theeditor and two anonymous referees for their constructive comments. The authors declare no competing financial interests.

\section{ORADiDS}

Paolo De Luca (1) https://orcid.org/0000-0002-04164622 


\section{Appendix}

Table of Notation Acronymsused within thetext and full definition.

\begin{tabular}{lll}
\hline Acronym & Definition & Unit \\
\hline A & basin area & $\mathrm{km}^{2}$ \\
AMAX & peak flow annual maximum & $\mathrm{m}^{3} \mathrm{~s}^{-1}$ \\
ARs & atmospheric rivers & $\mathrm{kg} \mathrm{m}^{-1} \mathrm{~s}^{-1}$ (integrated horizontal water vapour transport, IVT) \\
$\mathrm{d}_{\text {max }}$ & thelast day of a multi-basin flooding episode, wherethe & day \\
& largest number of basins recorded their AMAX & \\
ETCs & extratropical cyclones & - \\
FY & Food Yield & $\mathrm{m}^{3} \mathrm{~s}^{-1} \mathrm{~km}^{-2}$ \\
F-Index & Food Index & - \\
GB & Great Britain & - \\
HAs & hydrometric areas & - \\
Jt & joiningtime & days \\
L & timewindow & days \\
LWTs & Lamb Weather Types & - \\
MBF & multi-basin flooding & - \\
mFY & multi-basin Food Yield & $\mathrm{m}^{3} \mathrm{~s}^{-1} \mathrm{~km}^{-2}$ \\
$n_{g}$ & metric with severity based on thenumber of basins & - \\
& concurrently reaching their AMAX within a aiven time & \\
& window & \\
SPI & Standardized Precipitation Index & units of standard deviation \\
$T_{p}$ & timeto peak & hours \\
TDA & total drained area & $\mathrm{km}^{2}$ \\
VSG & Very Severe Gales & $\mathrm{G}>50$ (G = gale eindex) [48] \\
\hline
\end{tabular}

\section{References}

[1] UNISDR(United Nations International Strategy for Disaster Reduction) 2015 Sendai framework for disaster risk reduction 2015-2030

[2] ASC 2016 UK Climate Change Risk Assessment 2017 Synthesis Report: priorities for the next fiveyears Adaptation Sub-Committe of the Committeon Climate Change, London

[3] Barker L, Hannaford J, Muchan K, Turner Sand Parry S 2016 Thewinter 2015/2016 floodsin theUK: a hydrological appraisal Weather 71 324-33

[4] Muchan K, Lewis M, Hannaford Jand Parry S 2015 The winter storms of 2013/2014 in the UK: hydrological responses and impacts Weather 70 55-61

[5] Parry S, Marsh T and Kendon M 2013 2012: From drought to floodsin England and Wales Weather 68 268-74

[6] Marsh T 2008 A hydrological overview of the summer 2007 floodsin England and Wales Weather 63 274-9

[7] Merz Rand Blöschl G 2003 A process typology of regional floods Water Resour. Res $391-20$

[8] Nied M, Schröter K, LüdtkeS, Dung Nguyen V and Merz B 2016 What are the hydro-meteorological controls on flood characteristics? J. Hydrol. 545 310-26

[9] Gaăl L, Szzolgay J, HlavčováK, Parajka J, Viglione A, Merz R and Blöschl G 2015 Dependence between flood peeks and volumes: a case study on climate and hydrological controls Hydrol. Sa. J 60 968-84

[10] Viglione A, Chirico G B, KommaJ, Woods R, Borga M and Blöschl G 2010 Quantifying space-time dynamics of flood event types $J$. Hydrol 394 213-29

[11] Blöschl G \&t al 2015 Increasing river floods: fiction or reality? Wiley Interdiscip. Rev. Water 2329-44

[12] Huntingford $C$ et al 2014 Potential influences on the United Kingdom'sfloods of winter 2013/14 Nat. Clim. Change4 769-77

[13] Schaller $\mathrm{N}$ et al 2016 Human influence on climatein the 2014 southern England winter floodsand their impacts Nat. Clim. Change6 627-34

[14] Mallakpour I and Villarini G 2015 Thechanging nature of flooding across the central United States Nat. Clim. Change5 250-4

[15] Merz B et al 2014 Floodsand dimate: emerging perspectives for flood risk assessment and management Nat. Hazards Earth Syst. Sa. 14 1921-42
[16] Kjeldsen T R and JonesD A 2009 A formal statistical mode for pooled analysis of extremefloods Hydrol. Res 40 465-80

[17] Cunderlik JM and Burn D H 2003 Non-stationary pooled flood frequency analysis $J$ Hydrol. 276 210-23

[18] Heffernan JE and Tawn JA 2004 A conditional approach for multivariate extreme values $J$. R. Stat. Soc Ser. B Stat. Methodd. 66 497-530

[19] Kef C, Svensson C and Tawn JA 2009 Spatial dependence in extreme river flows and precipitation for Great Britain $J$. Hydrol. 378 240-52

[20] Kef C, Tawn JA and Lamb R 2013 Estimating the probability of widespread flood events Environmetrics24 13-21

[21] DeWaal D J, Van Gelder PH A JM and Nel A 2007 Estimating joint tail probabilities of river discharges through the logistic copula Environmetrics18 621-31

[22] Chen L, Sngh V P, Shenglian G, Hao Z and Li T 2012 Flood coincidence risk analysis using multivariate copula functions $J$ Hydrol. Eng. 17742-55

[23] Wyncoll D and Gouldby B 2013 Integrating a multivariate extreme value method within asystem flood risk analysis model $J$. Food Risk Manage 8 145-60

[24] Qu Y, Dodov B, Jain V and Hautaniemi T 2010 An inland flood loss estimation model for Great Britain British Hydrological Society 3rd International Symposium, Managing Consequenoes of a Changing Global Environment, 19-23 July

[25] Sampson C C, Fewtrell T J, O'Loughlin F, Pappenberger F, BatesPB, Frør JE and ClokeH L 2014 Theimpact of uncertain precipitation data on insurance loss estimates using aflood catastrophe model Hydrol. Earth Syst. Sa. 18 2305-24

[26] Ghizzoni T, Roth G and Rudari R 2012 Multisite flooding hazard assessment in the upper Mississippi river $J$ Hydrol. 412-413 101-13

[27] Ghizzoni T, Roth G and Rudari R 2010 Multivariate skew-t approach to the design of accumulation risk scenarios for the flooding hazard Adv. Water Resour. 33 1243-55

[28] Cameron D S, Beven K J, Tawn J, Blazkova Sand Naden P 1999 Flood frequency estimation by continuoussimulation for a gauged upland catchment (with uncertainty) J. Hydrol. 219 169-87

[29] Lamb R 2006 Rainfall-runoff modelling for flood frequency estimation Encyd. Hydrolog. Soi. 11125

[30] Lamb R, Keef C, Tawn J, Laeger S, Meadowcroft I, Surendran S. Dunning P and Bastone C 2010 A new method to assess the risk of local and widespread flooding on rivers and coasts $J$. Food Risk Manage 3 323-36 
[31] Uhlemann S, Thieken A H and Merz B 2010 A consistent set of trans-basin floods in Germany between 1952-2002 Hydrol. Earth Syst. Sa. 14 1277-95

[32] Uhlemann S, Thiken A H and Merz B 2014 A quality assessment framework for natural hazard event documentation: application to trans-basin flood reports in Germany Nat. Hazards Earth Syst. Sai. 14 189-208

[33] Jongman B, Hochrainer-Stigler S, Feyen L, Aerts JC JH, Mechler R, Botzen W JW, Bouwer L M, Pflug G, Rojas R and Ward PJ 2014 Increasing stress on disaster-risk finance due to large floods Nat. Clim. Change4 264-8

[34] Lavers D A, Allan RP, Wood EF, Villarini G, Brayshaw D J and Wade A J 2011 Winter floods in Britain are connected to atmospheric rivers Geophys Res Lett. 38 1-8

[35] Hillier JK, Macdonald N, Leckebusch G C and Stavrinides A 2015 Interactionsbetween apparently 'primary' weather-driven hazards and their cost Environ. Res Let. 10104003

[36] Perry A H $1980 \mathrm{~A}$ noteon the south Wales floods of late December 1979 Weather 35 106-9

[37] Kelman I 2001 Theautumn 2000 floods in England and flood management Weather 56 346-60

[38] Marsh T Jand DaleM 2002 TheUK floods of 2000-2001: a hydrometeorological appraisal Water Environ. J 16 180-8

[39] Environment Agency 2003 New Year Floods FoodLink

[40] Meteorological Office 1992 Monthly Weather Report 109

[41] HulmeM 1997 The climatein theUK from November 1994 to October 1995 Weather 52 242-57

[42] Watkins Sand Whytel 2008 Extreme flood events in upland catchmentsin Cumbria since 1600: the evidence of historical records North West Geogr. 81

[43] Wilby RL and Quinn N W 2013 Reconstructing multi-decadal variations in fluvial flood risk using atmospheric circulation patterns $J$. Hydrol. 487 109-21

[44] Kendon M and McCarthy M 2015 TheUK'swet and stormy winter of 2013/2014 Weather 70 40-7

[45] Wallingford Hydrosolutions 2016 The Revitalised Flood Hydrograph Model ReFH 2.2: Technical Guidanœe (Wallingford: Wallingford Hydrolsolutions and the Centrefor Ecology \& Hydrology)

[46] Wallingford: Centrefor Ecology \& Hydrology 2009 FEH CD-ROM 3

[47] Lamb H H 1972 British Is ses weather types and a register of the daily sequence of circulation patterns Geophysical Memoirs vol 116 (London: HMSO)

[48] JonesP D, Harpham C and BriffaK R 2013 Lamb weather types derived from reanalysis products Int. J. Climatol. 33 1129-39

[49] Lavers D A, Allan RP, Villarini G, Lloyd-HughesB, Brayshaw $D$ Jand Wade A J 2013 Future changes in atmospheric rivers and their implicationsfor winter flooding in Britain Environ. Res Lett. 8034010

[50] Brands S, Gutiérrez JM and San-Martín D 2017 Twentieth-century atmospheric river activity along the west coasts of Europe and North America: algorithm formulation, reanalysis uncertainty and linksto atmospheric circulation patterns Clim. Dyn. 48 2771-95

[51] DœD P \& al 2011 TheERA-Interim reanalysis: configuration and performance of the data assimilation system $Q$. J. $R$ Meteord. Soc. 137 553-97

[52] Berghuijs W R, WoodsRA, Hutton C Jand Svapalan M 2016 Dominant flood generating mechanisms across theUnited States Geophys Res Lett. 43 4382-90

[53] Mcke T B, Doesken N Jand Kleist J 1993 Therelationship of drought frequency and duration to timescales 8 th AMS Conferenceon Applied on Climatology, 17-22 vanuary

[54] McKeT B, Doesken N Jand Kleist J 1995 Drought monitoring with multipletimescales 9th AMSConferencon Applied Climatology, 15-20 January

[55] Seiler RA, Hayes M and Bressan L 2002 Using the standardized precipitation index for flood risk monitoring Int J. Climatol. 22 1365-76
[56] Du J, Fang J, Xu W and Shi P 2013 Analysis of dry/wet conditionsusing the standardized precipitation index and its potential usefulness for drought/flood monitoring in Hunan Province, China Stoch. Environ. Res Risk Assess 27 $377-87$

57] Wang Y, Chen X, Chen Y, Liu M and Gao L 2015 Food/drought event identification using an effective indicator based on the correlations between multiple time scales of the Standardized Precipitation Index and river discharge Theor. Appl. Climatol. 128 159-68

[58] Tanguy M, Kral F, Fry M, Svensson $\mathrm{C}$ and Hannaford J 2015 Standardised Precipitation Index time series for Integrated Hydrological Units Hydrometric Areas 1961-2012 NERC Environmental Information Data Centre

59] McCarthy M, SpillaneS, Walsh Sand Kendon M 2016 The meteorology of the exceptional winter of 2015/2016 across the UK and Ireland Weather 71 305-13

[60] Befort D J, Wild S, Kruschke T, Ulbrich U and Leckebusch G C 2016 Different long-term trends of extra-tropical cyclones and windstorms in ERA-20C and NOAA-20CR reanalyses Atmos Sai. Lett. 17 586-95

[61] Wild S, Befort D Jand Leckebusch G C 2015 Was the extreme storm season in winter 2013/14 over the North Atlantic and the United Kingdom triggered by changes in the West Pacific warm pool? Bull. Am. Meteord. Soc. 96 29-34

[62] Matthews T, Murphy C, Wilby RL and Harrigan S 2014 Stormiest winter on record for Ireland and UK Nat. Clim. Change $4738-40$

[63] Priestley M D K, Pinto JG, Dacre H F and Shaffrey L C 2017 The role of cyclone clustering during thestormy winter of 2013/2014 Weather 72 187-92

[64] Pattison I and LaneSN 2012 Therelationship between Lamb weather types and long-term changes in flood frequency, River Eden, UK Int. J. Climatol. 32 1971-89

[65] Hannaford Jand Marsh T J 2008 High-flow and flood trends in a network of undisturbed catchmentsin the UK Int. J. Climatol. 28 1325-38

[66] Matthews T, Murphy C, Wilby RL and Harrigan S 2016 A cyclone climatology of the British-Irish Isles 1871-2012 Int. J Climatol. 36 1299-312

[67] Grimaldi S, Petroselli A, Tauro F and Porfiri M 2012 Time of concentration: a paradox in modern hydrology Hydrol. Sa. J. 2 217-28

[68] Pinto JG, Zacharias S, Fink A H, Leckebusch G C and Ulbrich $\cup 2009$ Factors contributing to the development of extreme North Atlantic cyclones and their relationship with theNAO Clim. Dyn. 32 711-37

[69] Burt T P, Jones PD and Howden N JK 2015 An analysis of rainfall across the British Isesin the 1870s Int. J. Climatol. 35 2934-47

[70] Donat M G, Leckebusch G C, Pinto JG and Ulbrich U 2010 Examination of wind storms over Central Europe with respect to circulation weather types and NAO phases Int. J Climatol. 30 1289-300

[71] HM Government 2016 National Flood Resilience Review $145 \mathrm{pp}$

[72] Wilby R L and Keenan R 2012 Adapting to flood risk under climatechange Prog. Phys Geogr. 36 348-78

[73] Ulbrich U, Leckebusch G C and Pinto JG 2009 Extratropica cyclonesin thepresent and future climate: a review Theor. Appl. Climatol. 96 117-31

[74] Zappa G, Shaffrey L C, Hodges K I, Sansom P G and Stephenson D B 2013 A multimodel assessment of future projections of north atlantic and european extratropical cyclones in the CMIP5 climate models $J$ Clim. 26 $5846-62$

[75] Donat M G, Leckebusch G C, Pinto JG and Ulbrich U 2010 European storminess and associated circulation weather types: future changes deduced from amulti-model ensemble of GCM simulations Clim. Res 42 27-43 


\section{References}

AghaKouchak, A., Huning, L.S., Mazdiyasni, O., Mallakpour, I., Chiang, F., Sadegh, M.,

Vahedifard, F., Moftakhari, H., 2018. How do natural hazards cascade to cause disasters?

Nature 561, 458-460. https://doi.org/10.1038/d41586-018-06783-6

Araya-Muñoz, D., Metzger, M.J., Stuart, N., Wilson, A.M.W., Carvajal, D., 2017. A spatial fuzzy

logic approach to urban multi-hazard impact assessment in Concepción, Chile. Sci. Total

Environ. 576, 508-519. https://doi.org/http://dx.doi.org/10.1016/j.scitotenv.2016.10.077

Arnell, N.W., Gosling, S.N., 2016. The impacts of climate change on river flood risk at the global scale. Clim. Change 134, 387-401. https://doi.org/10.1007/s10584-014-1084-5

ASC, 2016. UK Climate Change Risk Assessment 2017 Synthesis Report: priorities for the next five years. Adapt. Sub-Committee Comm. Clim. Chang. London.

Ashraful Islam, M., Mitra, D., Dewan, A., Akhter, S.H., 2016. Coastal multi-hazard vulnerability assessment along the Ganges deltaic coast of Bangladesh-A geospatial approach. Ocean Coast.

Manag. 127, 1-15. https://doi.org/http://dx.doi.org/10.1016/j.ocecoaman.2016.03.012

Asprone, D., Jalayer, F., Prota, A., Manfredi, G., 2010. Proposal of a probabilistic model for multihazard risk assessment of structures in seismic zones subjected to blast for the limit state of collapse. Struct. Saf. 32, 25-34. https://doi.org/http://dx.doi.org/10.1016/j.strusafe.2009.04.002 Baldwin, M.P., Gray, L.J., Dunkerton, T.J., Hamilton, K., Haynes, P.H., Randel, W.J., Holton, J.R., Alexander, M.J., Hirota, I., Horinouchi, T., Jones, D.B.A., Kinnersley, J.S., Marquardt, C., Sato, K., Takahashi, M., 2001. The quasi-biennial oscillation. Rev. Geophys. 39, 179-229. https://doi.org/10.1029/1999RG000073

Barker, L., Hannaford, J., Muchan, K., Turner, S., Parry, S., 2016. The winter 2015/2016 floods in the UK: a hydrological appraisal. Weather 71, 324-333. https://doi.org/10.1002/wea.2822

Barnston, A.G., Livezey, R.E., 1987. Classification, Seasonality and Persistence of Low-Frequency Atmospheric Circulation Patterns. Mon. Weather Rev.

Barredo, J.I., 2007. Major flood disasters in Europe: 1950-2005. Nat. Hazards 42, 125-148. https://doi.org/10.1007/s11069-006-9065-2

Barriopedro, D., Fischer, E.M., Luterbacher, J., Trigo, R.M., García-Herrera, R., 2011. The Hot

Summer of 2010: Redrawing the Temperature Record Map of Europe. Science (80-. ). 332, 220 LP - 224. https://doi.org/10.1126/science.1201224

Bastin, J.-F., Clark, E., Elliott, T., Hart, S., van den Hoogen, J., Hordijk, I., Ma, H., Majumder, S., Manoli, G., Maschler, J., Mo, L., Routh, D., Yu, K., Zohner, C.M., Crowther, T.W., 2019. 
Understanding climate change from a global analysis of city analogues. PLoS One 14, e0217592.

Bastos, A., Gouveia, C.M., Trigo, R.M., Running, S.W., 2014. Analysing the spatio-temporal impacts of the 2003 and 2010 extreme heatwaves on plant productivity in Europe.

Biogeosciences 11, 3421-3435. https://doi.org/10.5194/bg-11-3421-2014

BBC, 2011. Horn of Africa sees "worst drought in 60 years." https://www.bbc.co.uk/news/worldafrica-13944550.

BBC, 2010a. Australia: Queensland floods spur more evacuations. https://www.bbc.co.uk/news/world-asia-pacific-12097280.

BBC, 2010b. Colombia flooding continues with thousands homeless.

https://www.bbc.co.uk/news/world-latin-america-12006568.

BBC, 2003. Floods bring miserable start to 2003. http://news.bbc.co.uk/1/hi/uk/2623729.stm.

Befort, D.J., Wild, S., Kruschke, T., Ulbrich, U., Leckebusch, G.C., 2016. Different long-term trends of extra-tropical cyclones and windstorms in ERA-20C and NOAA-20CR reanalyses. Atmos.

Sci. Lett. 17, 586-595. https://doi.org/10.1002/asl.694

Ben-Ari, T., Boé, J., Ciais, P., Lecerf, R., Van der Velde, M., Makowski, D., 2018. Causes and implications of the unforeseen 2016 extreme yield loss in the breadbasket of France. Nat.

Commun. 9, 1627. https://doi.org/10.1038/s41467-018-04087-X

Berghuijs, W.R., Allen, S.T., Harrigan, S., Kirchner, J.W., 2019. Growing spatial scales of synchronous river flooding in Europe. Geophys. Res. Lett. 0.

https://doi.org/10.1029/2018GL081883

Berton, R., Driscoll, C.T., Adamowski, J.F., 2017. The near-term prediction of drought and flooding conditions in the northeastern United States based on extreme phases of AMO and NAO. J.

Hydrol. 553, 130-141. https://doi.org/https://doi.org/10.1016/j.jhydrol.2017.07.041

Blenkinsop, S., Chan, S.C., Kendon, E.J., Roberts, N.M., Fowler, H.J., 2015. Temperature influences on intense UK hourly precipitation and dependency on large-scale circulation. Environ. Res.

Lett. 10, 54021. https://doi.org/10.1088/1748-9326/10/5/054021

Blöschl, G., Gaál, L., Hall, J., Kiss, A., Komma, J., Nester, T., Parajka, J., Perdigão, R.A.P., Plavcová, L., Rogger, M., Salinas, J.L., Viglione, A., 2015. Increasing river floods: fiction or reality? Wiley Interdiscip. Rev. Water 2, 329-344. https://doi.org/10.1002/wat2.1079

Blöschl, G., Hall, J., Parajka, J., Perdigão, R.A.P., Merz, B., Arheimer, B., Aronica, G.T., Bilibashi, A., Bonacci, O., Borga, M., Ivan, Č., Castellarin, A., Chirico, G.B., 2017. Changing climate shifts timing of European floods. Science (80-. ). 357, 588-590.

https://doi.org/10.1126/science.aan2506 
Bonferroni, C., 1936. Teoria statistica delle classi e calcolo delle probabilita. Pubbl. del R Ist. Super. di Sci. Econ. e Commericiali di Firenze 8, 3-62.

Bordi, I., Fraedrich, K., Sutera, A., 2009. Observed drought and wetness trends in Europe: an update. Hydrol. Earth Syst. Sci. 13, 1519-1530. https://doi.org/10.5194/hess-13-1519-2009

Bovolo, I.C., Abele, S.J., Bathurst, J.C., Caballero, D., Ciglan, M., Eftichidis, G., Simo, B., 2009. A distributed framework for multi-risk assessment of natural hazards used to model the effects of forest fire on hydrology and sediment yield. Comput. Geosci. 35, 924-945. https://doi.org/http://dx.doi.org/10.1016/j.cageo.2007.10.010

Brandimarte, L., Di Baldassarre, G., Bruni, G., D’Odorico, P., Montanari, A., 2011. Relation Between the North-Atlantic Oscillation and Hydroclimatic Conditions in Mediterranean Areas.

Water Resour. Manag. 25, 1269-1279. https://doi.org/10.1007/s11269-010-9742-5

Brands, S., Gutiérrez, J.M., San-Martín, D., 2016. Twentieth-century atmospheric river activity along the west coasts of Europe and North America: algorithm formulation, reanalysis uncertainty and links to atmospheric circulation patterns. Clim. Dyn. 1-25. https://doi.org/10.1007/s00382-016-3095-6

Briffa, K.R., van der Schrier, G., Jones, P.D., 2009. Wet and dry summers in Europe since 1750: evidence of increasing drought. Int. J. Climatol. 29, 1894-1905. https://doi.org/10.1002/joc.1836

Brown, D.W., Moin, S.M.A., Nicolson, M.L., 1997. A comparison of flooding in Michigan and Ontario: "Soft" data to support "soft" water management approaches. Can. Water Resour. J. 22, $125-139$.

Burt, S., 2016. New extreme monthly rainfall totals for the United Kingdom and Ireland: December 2015. Weather 71. https://doi.org/10.1002/wea.2822

Burt, S., Kendon, M., 2016. December 2015 - an exceptionally mild month in the United Kingdom. Burt, S., McCarthy, M., Kendon, M., Hannaford, J., 2016. Cumbrian floods, 5/6 December 2015. Weather 71, 36-37. https://doi.org/10.1002/wea.2704

Burt, T.P., Ferranti, E.J.S., 2012. Changing patterns of heavy rainfall in upland areas: A case study from northern England. Int. J. Climatol. 32, 518-532. https://doi.org/10.1002/joc.2287

Burt, T.P., Howden, N.J.K., 2013. North Atlantic Oscillation amplifies orographic precipitation and river flow in upland Britain. Water Resour. Res. 49, 3504-3515. https://doi.org/10.1002/wrcr.20297

Burt, T.P., Jones, P.D., Howden, N.J.K., 2015. An analysis of rainfall across the British Isles in the 1870s. Int. J. Climatol. 35, 2934-2947. https://doi.org/10.1002/joc.4184

C3S, 2017. ERA5: Fifth generation of ECMWF atmospheric reanalyses of the global climate 
[WWW Document]. Copernicus Clim. Chang. Serv. Clim. Data Store (CDS), date access 10/10/2019. URL https://cds.climate.copernicus.eu/cdsapp\#!/home

Cai, W., Rensch, P., 2012. The 2011 southeast Queensland extreme summer rainfall: A confirmation of a negative Pacific Decadal Oscillation phase? Geophys. Res. Lett. 39. https://doi.org/10.1029/2011GL050820

Cameron, D.., Beven, K.., Tawn, J., Blazkova, S., Naden, P., 1999. Flood frequency estimation by continuous simulation for a gauged upland catchment (with uncertainty). J. Hydrol. 219, 169187. https://doi.org/10.1016/S0022-1694(99)00057-8

Cayan, D.R., Redmond, K.T., Riddle, L.G., 1999. ENSO and Hydrologic Extremes in the Western United States. J. Clim. 12, 2881-2893. https://doi.org/10.1175/15200442(1999)012<2881:EAHEIT>2.0.CO;2

CCRA, 2016. UK Climate Change Risk Assessment 2017. Synthesis report: priorities for the next five years.

Chan, S.C., Kendon, E.J., Fowler, H.J., Blenkinsop, S., Roberts, N.M., 2014. Projected increases in summer and winter UK sub-daily precipitation extremes from high-resolution regional climate models. Environ. Res. Lett. 9, 84019. https://doi.org/10.1088/1748-9326/9/8/084019 Changnon, S.A., Pielke, R.A., Changnon, D., Sylves, R.T., Pulwarty, R., 2000. Human Factors Explain the Increased Losses from Weather and Climate Extremes. Bull. Am. Meteorol. Soc. 81, 437-442. https://doi.org/10.1175/1520-0477(2000)081<0437:HFETIL>2.3.CO;2

Chen, D., 2000. A monthly circulation climatology for Sweden and its application to a winter temperature case study. Int. J. Climatol. 20, 1067-1076. https://doi.org/10.1002/10970088(200008)20:10<1067::AID-JOC528>3.0.CO;2-Q

Chen, L., Singh, V.P., Shenglian, G., Hao, Z., Li, T., 2012. Flood Coincidence Risk Analysis Using Multivariate Copula Functions. J. Hydrol. Eng. 17, 742-755. https://doi.org/10.1061/(ASCE)HE.1943-5584.0000504

Chen, T., Zhang, H., Chen, X., Hagan, D.F., Wang, G., Gao, Z., Shi, T., 2017. Robust drying and wetting trends found in regions over China based on Köppen climate classifications. J.

Geophys. Res. Atmos. 122, 4228-4237. https://doi.org/10.1002/2016JD026168

Cohen, J., Pfeiffer, K., Francis, J.A., 2018. Warm Arctic episodes linked with increased frequency of extreme winter weather in the United States. Nat. Commun. 9, 869. https://doi.org/10.1038/s41467-018-02992-9

Cohen, J., Screen, J.A., Furtado, J.C., Barlow, M., Whittleston, D., Coumou, D., Francis, J., Dethloff, K., Entekhabi, D., Overland, J., Jones, J., 2014. Recent Arctic amplification and extreme mid-latitude weather. Nat. Geosci. 7, 627. 
Coles, S., 2001. An Introduction to Statistical Modeling of Extreme Values, 1st ed. Springer-Verlag London. https://doi.org/10.1007/978-1-4471-3675-0

Collet, L., Harrigan, S., Prudhomme, C., Formetta, G., Beevers, L., 2018. Future hot-spots for hydrohazards in Great Britain: a probabilistic assessment. Hydrol. Earth Syst. Sci. 22, 5387-5401. https://doi.org/10.5194/hess-22-5387-2018

Collier, C.G., 2007. Flash flood forecasting: What are the limits of predictability? Q. J. R. Meteorol. Soc. 133, 3-23. https://doi.org/10.1002/qj.29

Compo, G.P., Whitaker, J.S., Sardeshmukh, P.D., Matsui, N., Allan, R.J., Yin, X., Gleason, B.E., Vose, R.S., Rutledge, G., Bessemoulin, P., Brönnimann, S., Brunet, M., Crouthamel, R.I., Grant, A.N., Groisman, P.Y., Jones, P.D., Kruk, M.C., Kruger, A.C., Marshall, G.J., Maugeri, M., Mok, H.Y., Nordli, Ø., Ross, T.F., Trigo, R.M., Wang, X.L., Woodruff, S.D., Worley, S.J., 2011. The Twentieth Century Reanalysis Project. Q. J. R. Meteorol. Soc. 137, 1-28. https://doi.org/10.1002/qj.776

Conticello, F., Cioffi, F., Merz, B., Lall, U., 2018. An event synchronization method to link heavy rainfall events and large-scale atmospheric circulation features. Int. J. Climatol. 38, 1421-1437. https://doi.org/10.1002/joc.5255

Conway, D., Jones, P.D., 1998. The use of weather types and air flow indices for GCM downscaling. J. Hydrol. 212-213, 348-361. https://doi.org/https://doi.org/10.1016/S0022-1694(98)00216-9 Cortesi, N., Gonzalez-Hidalgo, J.C., Trigo, R.M., Ramos, A.M., 2014. Weather types and spatial variability of precipitation in the Iberian Peninsula. Int. J. Climatol. 34, 2661-2677. https://doi.org/10.1002/joc.3866

Cortesi, N., Trigo, R.M., Gonzalez-Hidalgo, J.C., Ramos, A.M., 2013. Modelling monthly precipitation with circulation weather types for a dense network of stations over Iberia. Hydrol.

Earth Syst. Sci. 17, 665-678. https://doi.org/10.5194/hess-17-665-2013

Coumou, D., Di Capua, G., Vavrus, S., Wang, L., Wang, S., 2018. The influence of Arctic amplification on mid-latitude summer circulation. Nat. Commun. 9, 2959.

https://doi.org/10.1038/s41467-018-05256-8

Crompton, R.P., McAneney, K.J., 2008. Normalised Australian insured losses from meteorological hazards: 1967-2006. Environ. Sci. Policy 11, 371-378. https://doi.org/https://doi.org/10.1016/j.envsci.2008.01.005

Crompton, R.P., Pielke Jr, R.A., McAneney, K.J., 2011. Emergence timescales for detection of anthropogenic climate change in US tropical cyclone loss data. Environ. Res. Lett. 6, 14003. https://doi.org/10.1088/1748-9326/6/1/014003

Cunderlik, J.M., Burn, D.H., 2003. Non-stationary pooled flood frequency analysis. J. Hydrol. 276, 
$210-223$.

Cutter, S.L., Barnes, L., Berry, M., Burton, C., Evans, E., Tate, E., Webb, J., 2008. A place-based model for understanding community resilience to natural disasters. Glob. Environ. Chang. 18, 598-606. https://doi.org/https://doi.org/10.1016/j.gloenvcha.2008.07.013

Dai, A., 2017. Dai Global Palmer Drought Severity Index (PDSI). Research Data Archive at the

National Center for Atmospheric Research, Computational and Information Systems

Laboratory. Accessed 23/04/2019. https://doi.org/10.5065/D6QF8R93

Dai, A., 2012. Increasing drought under global warming in observations and models. Nat. Clim. Chang. 3, 52.

Dai, A., 2011a. Drought under global warming: A review. Wiley Interdiscip. Rev. Clim. Chang. 2, 45-65. https://doi.org/10.1002/wcc.81

Dai, A., 2011b. Characteristics and trends in various forms of the Palmer Drought Severity Index during 1900-2008. J. Geophys. Res. Atmos. 116. https://doi.org/10.1029/2010JD015541

Dai, A., Trenberth, K.E., Qian, T., 2004. A Global Dataset of Palmer Drought Severity Index for 1870-2002: Relationship with Soil Moisture and Effects of Surface Warming. J.

Hydrometeorol. 5, 1117-1130. https://doi.org/10.1175/JHM-386.1

Dai, A., Wigley, T.M.L., 2000. Global patterns of ENSO-induced precipitation. Geophys. Res. Lett. 27, 1283-1286. https://doi.org/10.1029/1999GL011140

Dall’Osso, F., Dominey-Howes, D., Moore, C., Summerhayes, S., Withycombe, G., 2014. The exposure of Sydney (Australia) to earthquake-generated tsunamis, storms and sea level rise: a probabilistic multi-hazard approach 4, 7401.

De Luca, P., Harpham, C., Wilby, R.L., Hillier, J.K., Franzke, C.L.E., Leckebusch, G.C., 2019a. Past and projected weather pattern persistence with associated multi-hazards in the British Isles.

EarthArXiv. https://doi.org/10.31223/osf.io/ghuzv

De Luca, P., Hillier, J.K., Wilby, R.L., Quinn, N.W., Harrigan, S., 2017. Extreme multi-basin flooding linked with extra-tropical cyclones. Environ. Res. Lett. 12, 114009. https://doi.org/10.1088/1748-9326/aa868e

De Luca, P., Messori, G., Faranda, D., Pons, F.M.E., 2019b. Dynamical Systems Theory Sheds New Light on Compound Climate Extremes in Europe and Eastern North America. EarthArXiv. https://doi.org/https://doi.org/10.31223/osf.io/qdn5w

De Luca, P., Messori, G., Wilby, R.L., Mazzoleni, M., Di Baldassarre, G., 2019c. Concurrent wet and dry hydrological extremes at the global scale. Earth Syst. Dynam. Discuss. 1-24. https://doi.org/10.5194/esd-2019-27

De Waal, D., Van Gelder, P. and Nel, A., 2007. Estimating joint tail probabilities of river discharges 
through the logistic copula. Environmetrics 18, 621-631. https://doi.org/10.1002/env Deangelis, R.J., Urban, J.B., Gburek, W.J., Contino, M.A., 1984. Precipitation and runoff on eight New England basins during extreme wet and dry periods. Hydrol. Sci. J. 29, 13-28. https://doi.org/10.1080/02626668409490919

Dee, D.P., Uppala, S.M., Simmons, A.J., Berrisford, P., Poli, P., Kobayashi, S., Andrae, U., Balmaseda, M.A., Balsamo, G., Bauer, P., Bechtold, P., Beljaars, A.C.M., van de Berg, L., Bidlot, J., Bormann, N., Delsol, C., Dragani, R., Fuentes, M., Geer, A.J., Haimberger, L., Healy, S.B., Hersbach, H., Hólm, E. V, Isaksen, L., Kållberg, P., Köhler, M., Matricardi, M., McNally, A.P., Monge-Sanz, B.M., Morcrette, J.-J., Park, B.-K., Peubey, C., de Rosnay, P., Tavolato, C., Thépaut, J.-N., Vitart, F., 2011. The ERA-Interim reanalysis: configuration and performance of the data assimilation system. Q. J. R. Meteorol. Soc. 137, 553-597.

https://doi.org/10.1002/qj.828

Demuzere, M., van Lipzig, N.P.M., 2010. A new method to estimate air-quality levels using a synoptic-regression approach. Part I: Present-day O3 and PM10 analysis. Atmos. Environ. 44, 1341-1355. https://doi.org/https://doi.org/10.1016/j.atmosenv.2009.06.029

Demuzere, M., Werner, M., van Lipzig, N.P.M., Roeckner, E., 2009. An analysis of present and future ECHAM5 pressure fields using a classification of circulation patterns. Int. J. Climatol. 29, 1796-1810. https://doi.org/10.1002/joc.1821

DFO, 2008. Dartmouth Flood Observatory, Global Active Archive of Large Flood Events [WWW Document]. http://www.dartmouth.edu/ floods/Archives/2003sum.htm.

Di Baldassarre, G., Martinez, F., Kalantari, Z., Viglione, A., 2017. Drought and flood in the Anthropocene: feedback mechanisms in reservoir operation. Earth Syst. Dyn. 8, 225-233. https://doi.org/10.5194/esd-8-225-2017

Di Baldassarre, G., Nohrstedt, D., Mård, J., Burchardt, S., Albin, C., Bondesson, S., Breinl, K., Deegan, F.M., Fuentes, D., Lopez, M.G., Granberg, M., Nyberg, L., Nyman, M.R., Rhodes, E., Troll, V., Young, S., Walch, C., Parker, C.F., 2018. An Integrative Research Framework to Unravel the Interplay of Natural Hazards and Vulnerabilities. Earth's Futur. 6, 305-310. https://doi.org/10.1002/2017EF000764

Di Capua, G., Coumou, D., 2016. Changes in meandering of the Northern Hemisphere circulation. Environ. Res. Lett. 11, 94028.

Dirmeyer, P.A., Brubaker, K.L., 1999. Contrasting evaporative moisture sources during the drought of 1988 and the flood of 1993. J. Geophys. Res. Atmos. 104, 19383-19397.

https://doi.org/10.1029/1999JD900222

Domínguez-Castro, F., García-Herrera, R., Vicente-Serrano, S.M., 2018. Wet and dry extremes in 
Quito (Ecuador) since the 17th century. Int. J. Climatol. 38, 2006-2014. https://doi.org/10.1002/joc.5312

Domínguez-Castro, F., Ramos, A.M., García-Herrera, R., Trigo, R.M., 2015. Iberian extreme precipitation 1855/1856: an analysis from early instrumental observations and documentary sources. Int. J. Climatol. 35, 142-153. https://doi.org/10.1002/joc.3973

Donat M. G., Leckebusch G. C., Pinto J. G., U.U., 2010. Examination of wind storms over Central Europe with respect to circulation weather types and NAO phases. Int. J. Climatol. 30, 12891300.

Donat, M.G., Leckebusch, G.C., Pinto, J.G., Ulbrich, U., 2010. European storminess and associated circulation weather types: future changes deduced from a multi-model ensemble of GCM simulations. Clim. Res. 42, 27-43. https://doi.org/10.3354/cr00853

Dong, X., Xi, B., Kennedy, A., Feng, Z., Entin, J.K., Houser, P.R., Schiffer, R.A., L’Ecuyer, T., Olson, W.S., Hsu, K., Liu, W.T., Lin, B., Deng, Y., Jiang, T., 2011. Investigation of the 2006 drought and 2007 flood extremes at the Southern Great Plains through an integrative analysis of observations. J. Geophys. Res. Atmos. 116. https://doi.org/10.1029/2010JD014776

Doswell, C.A., Brooks, H.E., Maddox, R.A., 1996. Flash Flood Forecasting: An Ingredients-Based Methodology. Weather Forecast. 11, 560-581. https://doi.org/10.1175/15200434(1996)011<0560:FFFAIB>2.0.CO;2

Du, J., Fang, J., Xu, W., Shi, P., 2013. Analysis of dry/wet conditions using the standardized precipitation index and its potential usefulness for drought/flood monitoring in Hunan Province,

China. Stoch. Environ. Res. Risk Assess. 27, 377-387. https://doi.org/10.1007/s00477-0120589-6

Eden, J.M., van Oldenborgh, G.J., Hawkins, E., Suckling, E.B., 2015. A global empirical system for probabilistic seasonal climate prediction. Geosci. Model Dev. 8, 3947-3973. https://doi.org/10.5194/gmd-8-3947-2015

Eiras-Barca, J., Lorenzo, N., Taboada, J., Robles, A., Miguez-Macho, G., 2018. On the relationship between atmospheric rivers, weather types and floods in Galicia (NW Spain). Nat. Hazards Earth Syst. Sci. 18, 1633-1645. https://doi.org/10.5194/nhess-18-1633-2018

Ekström, M., Gutmann, E.D., Wilby, R.L., Tye, M.R., Kirono, D.G.C., 2018. Robustness of hydroclimate metrics for climate change impact research. Wiley Interdiscip. Rev. Water 5, e1288. https://doi.org/10.1002/wat2.1288

Emanuel, K., 2005. Increasing destructiveness of tropical cyclones over the past 30 years. Nature 436, 686.

Emanuel, K.A., 2013. Downscaling CMIP5 climate models shows increased tropical cyclone activity 
over the 21st century. Proc. Natl. Acad. Sci. 110, 12219 LP - 12224.

https://doi.org/10.1073/pnas.1301293110

Emerton, R., Cloke, H.L., Stephens, E.M., Zsoter, E., Woolnough, S.J., Pappenberger, F., 2017.

Complex picture for likelihood of ENSO-driven flood hazard. Nat. Commun. 8, 14796.

Emerton, R.E., Stephens, E.M., Cloke, H.L., 2019. What is the most useful approach for forecasting hydrological extremes during El Niño? Environ. Res. Commun. 1, 31002.

https://doi.org/10.1088/2515-7620/ab114e

Enfield, D.B., Mestas-Nuñez, A.M., Trimble, P.J., 2001. The Atlantic Multidecadal Oscillation and its relation to rainfall and river flows in the continental U.S. Geophys. Res. Lett. 28, 2077-

2080. https://doi.org/10.1029/2000GL012745

Environment Agency, 2003. New Year Floods. FloodLink.

Eyring, V., Bony, S., Meehl, G.A., Senior, C.A., Stevens, B., Stouffer, R.J., Taylor, K.E., 2016.

Overview of the Coupled Model Intercomparison Project Phase 6 (CMIP6) experimental design and organization. Geosci. Model Dev. 9, 1937-1958. https://doi.org/10.5194/gmd-9-1937-2016

Ezer, T., Atkinson, L.P., 2014. Accelerated flooding along the U.S. East Coast: On the impact of sealevel rise, tides, storms, the Gulf Stream, and the North Atlantic Oscillations. Earth's Futur. 2, 362-382. https://doi.org/10.1002/2014EF000252

Faranda, D., Alvarez-Castro, M.C., Messori, G., Rodrigues, D., Yiou, P., 2019. The hammam effect or how a warm ocean enhances large scale atmospheric predictability. Nat. Commun. 10, 1316. https://doi.org/10.1038/s41467-019-09305-8

Faranda, D., Messori, G., Alvarez-Castro, M.C., Yiou, P., 2017a. Dynamical properties and extremes of Northern Hemisphere climate fields over the past 60 years. Nonlin. Process. Geophys. 24, 713-725. https://doi.org/10.5194/npg-24-713-2017

Faranda, D., Messori, G., Yiou, P., 2017b. Dynamical proxies of North Atlantic predictability and extremes. Sci. Rep. 7, 41278.

Farnham, D.J., Doss-Gollin, J., Lall, U., 2018. Regional Extreme Precipitation Events: Robust Inference From Credibly Simulated GCM Variables. Water Resour. Res. 54, 3809-3824. https://doi.org/10.1002/2017WR021318

Fernández-González, S., del Río, S., Castro, A., Penas, A., Fernández-Raga, M., Calvo, A.I., Fraile, R., 2012. Connection between NAO, weather types and precipitation in León, Spain (19482008). Int. J. Climatol. 32, 2181-2196. https://doi.org/10.1002/joc.2431

Fernandez-Raga, M., Castro, A., Marcos, E., Palencia, C., Fraile, R., 2017. Weather types and rainfall microstructure in Leon, Spain. Int. J. Climatol. 37, 1834-1842.

https://doi.org/10.1002/joc.4816 
Fischer, E.M., Beyerle, U., Knutti, R., 2013. Robust spatially aggregated projections of climate extremes. Nat. Clim. Chang. 3, 1033.

Fischer, E.M., Knutti, R., 2016. Observed heavy precipitation increase confirms theory and early models. Nat. Clim. Chang. 6, 986.

Fischer, E.M., Knutti, R., 2015. Anthropogenic contribution to global occurrence of heavyprecipitation and high-temperature extremes. Nat. Clim. Chang. 5, 560.

Fischer, E.M., Schär, C., 2010. Consistent geographical patterns of changes in high-impact European heatwaves. Nat. Geosci. 3, 398.

Fleig, A.K., Tallaksen, L.M., Hisdal, H., Stahl, K., Hannah, D.M., 2010. Inter-comparison of weather and circulation type classifications for hydrological drought development. Phys. Chem.

Earth, Parts A/B/C 35, 507-515. https://doi.org/https://doi.org/10.1016/j.pce.2009.11.005

Forzieri, G., Feyen, L., Russo, S., Vousdoukas, M., Alfieri, L., Outten, S., Migliavacca, M., Bianchi, A., Rojas, R., Cid, A., 2016. Multi-hazard assessment in Europe under climate change. Clim. Change 137, 105-119. https://doi.org/10.1007/s10584-016-1661-x

Fowler, H.J., Kilsby, C.G., 2002a. Precipitation and the North Atlantic Oscillation: a study of climatic variability in northern England. Int. J. Climatol. 22, 843-866. https://doi.org/10.1002/joc.765

Fowler, H.J., Kilsby, C.G., 2002b. A weather-type approach to analysing water resource drought in the Yorkshire region from 1881 to 1998. J. Hydrol. 262, 177-192. https://doi.org/https://doi.org/10.1016/S0022-1694(02)00034-3

Fowler, H.J., Kilsby, C.G., O'Connell, P.E., 2000. A stochastic rainfall model for the assessment of regional water resource systems under changed climatic condition. Hydrol. Earth Syst. Sci. 4, 263-281. https://doi.org/10.5194/hess-4-263-2000

Francis, J., Skific, N., 2015. Evidence linking rapid Arctic warming to mid-latitude weather patterns. Philos. Trans. R. Soc. London A Math. Phys. Eng. Sci. 373. https://doi.org/10.1098/rsta.2014.0170

Francis, J.A., 2017. Why Are Arctic Linkages to Extreme Weather Still up in the Air? Bull. Am. Meteorol. Soc. 98, 2551-2557. https://doi.org/10.1175/BAMS-D-17-0006.1

Francis, J.A., Vavrus, S.J., 2015. Evidence for a wavier jet stream in response to rapid Arctic warming. Environ. Res. Lett. 10, 14005.

Francis, J.A., Vavrus, S.J., 2012. Evidence linking Arctic amplification to extreme weather in midlatitudes. Geophys. Res. Lett. 39. https://doi.org/10.1029/2012GL051000

Francis, J.A., Vavrus, S.J., Cohen, J., 2017. Amplified Arctic warming and mid-latitude weather: new perspectives on emerging connections. Wiley Interdiscip. Rev. Clim. Chang. 8, e474. 


\section{https://doi.org/10.1002/wcc.474}

Franzke, C.L.E., 2013. Persistent regimes and extreme events of the North Atlantic atmospheric circulation. Philos. Trans. R. Soc. London A Math. Phys. Eng. Sci. 371.

https://doi.org/10.1098/rsta.2011.0471

Gaál, L., Szolgay, J., Kohnová, S., Hlavčová, K., Parajka, J., Viglione, A., Merz, R., Blöschl, G.,

2015. Dependence between flood peaks and volumes: a case study on climate and hydrological controls. Hydrol. Sci. J. 60:6, 968-984. https://doi.org/10.1016/j.jhydrol.2010.05.047

Gabric, A.J., Cropp, R.A., McTainsh, G.H., Johnston, B.M., Butler, H., Tilbrook, B., Keywood, M., 2010. Australian dust storms in 2002-2003 and their impact on Southern Ocean

biogeochemistry. Global Biogeochem. Cycles 24. https://doi.org/10.1029/2009GB003541

Gagniuc, P.A., 2017. Markov Chains: From Theory to Implementation and Experimentation. USA,

NJ: John Wiley \& Sons. https://doi.org/10.1002/9781119387596

Gallina, V., Torresan, S., Critto, A., Sperotto, A., Glade, T., Marcomini, A., 2016. A review of multirisk methodologies for natural hazards: Consequences and challenges for a climate change impact assessment. J. Environ. Manage. 168, 123-132.

García-Valdecasas Ojeda, M., Gámiz-Fortis, S.R., Castro-Díez, Y., Esteban-Parra, M.J., 2017.

Evaluation of WRF capability to detect dry and wet periods in Spain using drought indices. J.

Geophys. Res. Atmos. 122, 1569-1594. https://doi.org/10.1002/2016JD025683

Ghizzoni, T., Roth, G., Rudari, R., 2012. Multisite flooding hazard assessment in the Upper

Mississippi River. J. Hydrol. 412-413, 101-113. https://doi.org/10.1016/j.jhydrol.2011.06.004

Ghizzoni, T., Roth, G., Rudari, R., 2010. Multivariate skew-t approach to the design of accumulation risk scenarios for the flooding hazard. Adv. Water Resour. 33, 1243-1255.

https://doi.org/10.1016/j.advwatres.2010.08.003

Gil-Guirado, S., Espín-Sánchez, J.-A., Del Rosario Prieto, M., 2016. Can we learn from the past?

Four hundred years of changes in adaptation to floods and droughts. Measuring the vulnerability in two Hispanic cities. Clim. Change 139, 183-200.

https://doi.org/10.1007/s10584-016-1768-0

Gill, J.C., Malamud, B.D., 2017. Anthropogenic processes, natural hazards, and interactions in a multi-hazard framework. Earth-Science Rev. 166, 246-269. https://doi.org/http://dx.doi.org/10.1016/j.earscirev.2017.01.002

Gill, J.C., Malamud, B.D., 2014. Reviewing and visualizing the interactions of natural hazards. Rev. Geophys. 52, 680-722. https://doi.org/10.1002/2013RG000445

Goodess, C.M., Palutikof, J.P., 1998. Development of daily rainfall scenarios for southeast Spain using a circulation-type approach to downscaling. Int. J. Climatol. 18, 1051-1083. 
https://doi.org/10.1002/(SICI)1097-0088(199808)18:10<1051::AID-JOC304>3.0.CO;2-1

Grimaldi, S., Petroselli, A., Tauro, F., Porfiri, M., Grimaldi, S., Petroselli, A., Tauro, F., Porfiri, M., 2016. Time of concentration : a paradox in modern hydrology 6667. https://doi.org/10.1080/02626667.2011.644244

Grundström, M., Hak, C., Chen, D., Hallquist, M., Pleijel, H., 2015a. Variation and co-variation of PM10, particle number concentration, NOx and NO2 in the urban air - Relationships with wind speed, vertical temperature gradient and weather type. Atmos. Environ. 120, 317-327.

https://doi.org/https://doi.org/10.1016/j.atmosenv.2015.08.057

Grundström, M., Tang, L., Hallquist, M., Nguyen, H., Chen, D., Pleijel, H., 2015b. Influence of atmospheric circulation patterns on urban air quality during the winter. Atmos. Pollut. Res. 6, 278-285. https://doi.org/https://doi.org/10.5094/APR.2015.032

Grünthal, G., Thieken, A.H., Schwarz, J., Radtke, K.S., Smolka, A., Merz, B., 2006. Comparative Risk Assessments for the City of Cologne - Storms, Floods, Earthquakes. Nat. Hazards 38, 2144. https://doi.org/10.1007/s11069-005-8598-0

Güneralp, B., Güneralp, İ., Liu, Y., 2015. Changing global patterns of urban exposure to flood and drought hazards. Glob. Environ. Chang. 31, 217-225. https://doi.org/https://doi.org/10.1016/j.gloenvcha.2015.01.002

Hall, J.I.M.W., Sayers, P.B., Dawson, R.J., 2005. National-scale Assessment of Current and Future Flood Risk in England and Wales 147-164.

Hamed, K.H., Ramachandra Rao, A., 1998. A modified Mann-Kendall trend test for autocorrelated data. J. Hydrol. 204, 182-196. https://doi.org/https://doi.org/10.1016/S0022-1694(97)00125-X Hamlet, A.F., Lettenmaier, D.P., 2007. Effects of 20th century warming and climate variability on flood risk in the western U.S. Water Resour. Res. 43. https://doi.org/10.1029/2006WR005099 Hannachi, A., Straus, D.M., Franzke, C.L.E., Corti, S., Woollings, T., 2017. Low-frequency nonlinearity and regime behavior in the Northern Hemisphere extratropical atmosphere. Rev.

Geophys. 55, 199-234. https://doi.org/10.1002/2015RG000509

Hannaford, J., Marsh, T.J., 2008. High-flow and flood trends in a network of undisturbed catchments in the UK. Int. J. Climatol. 28, 1325-1338. https://doi.org/10.1002/joc

Hao, Z., Hao, F., Singh, V.P., Zhang, X., 2018. Changes in the severity of compound drought and hot extremes over global land areas. Environ. Res. Lett. 13, 124022.

https://doi.org/10.1088/1748-9326/aaee96

Harding, K.J., Snyder, P.K., 2015. The Relationship between the Pacific-North American Teleconnection Pattern, the Great Plains Low-Level Jet, and North Central U.S. Heavy Rainfall Events. J. Clim. 28, 6729-6742. https://doi.org/10.1175/JCLI-D-14-00657.1 
Harrington, L.J., Otto, F.E.L., Cowan, T., Hegerl, G.C., 2019. Circulation analogues and uncertainty in the time-evolution of extreme event probabilities: evidence from the 1947 Central European heatwave. Clim. Dyn. 53, 2229-2247. https://doi.org/10.1007/s00382-019-04820-2 Harris, A.J., Vallance, J.W., Kimberly, P., Rose, W.I., Matías, O., Bunzendahl, E., Flynn, L.P., Garbeil, H., 2006. Downstream aggradation owing to lava dome extrusion and rainfall runoff at Volcan Santiaguito, Guatemala. Geol. Soc. Am. Spec. Pap. 412, 85-104.

Harrison, A.M., Plim, J.F.M., Harrison, M., Jones, L.D., Culshaw, M.G., 2012. The relationship between shrink-swell occurrence and climate in south-east England. Proc. Geol. Assoc. 123, 556-575. https://doi.org/http://dx.doi.org/10.1016/j.pgeola.2012.05.002

Heffernan, J.E., Tawn, J.A., 2004. A conditional approach for multivariate extreme values. J. R. Stat. Soc. Ser. B Stat. Methodol. 66, 497-530. https://doi.org/10.1111/j.1467-9868.2004.02050.x Hellström, C., 2005. Atmospheric conditions during extreme and non-extreme precipitation events in Sweden. Int. J. Climatol. 25, 631-648. https://doi.org/10.1002/joc.1119

Hess, P., Brezowsky, H., 1952. Katalog der Großwetterlagen Europas.Berichte des Deutschen Wetterdienstes in der US-Zone 33. DeutscherWetterdienst in d. US-Zone: Bad Kissingen. Hewitt, K., Burton, I., 1971. The hazardousness of a place: a regional ecology of damaging events. Res. Publ. 6.

Hillier, J.K., Macdonald, N., Leckebusch, G.C., Stavrinides, a, 2015. Interactions between apparently 'primary' weather-driven hazards and their cost. Environ. Res. Lett. 10, 104003. https://doi.org/10.1088/1748-9326/10/10/104003

Hirabayashi, Y., Mahendran, R., Koirala, S., Konoshima, L., Yamazaki, D., Watanabe, S., Kim, H., Kanae, S., 2013. Global flood risk under climate change. Nat. Clim. Chang. 3, 816. HM Government, 2016. National Flood Resilience Review 145.

Hochman, A., Alpert, P., Harpaz, T., Saaroni, H., Messori, G., 2019. A new dynamical systems perspective on atmospheric predictability: Eastern Mediterranean weather regimes as a case study. Sci. Adv. 5, eaau0936. https://doi.org/10.1126/sciadv.aau0936

Hodgkins, G.A., 2009. Streamflow changes in Alaska between the cool phase (1947-1976) and the warm phase (1977-2006) of the Pacific Decadal Oscillation: The influence of glaciers. Water

Resour. Res. 45. https://doi.org/10.1029/2008WR007575

Hodgkins, G.A., Whitfield, P.H., Burn, D.H., Hannaford, J., Renard, B., Stahl, K., Fleig, A.K., Madsen, H., Mediero, L., Korhonen, J., Murphy, C., Wilson, D., 2017. Climate-driven variability in the occurrence of major floods across North America and Europe. J. Hydrol. 552, 704-717. https://doi.org/https://doi.org/10.1016/j.jhydrol.2017.07.027

Horridge, M., Madden, J., Wittwer, G., 2005. The impact of the 2002-2003 drought on Australia. J. 
Policy Model. 27, 285-308. https://doi.org/https://doi.org/10.1016/j.jpolmod.2005.01.008 Huang, S., Krysanova, V., Hattermann, F., 2015. Projections of climate change impacts on floods and droughts in Germany using an ensemble of climate change scenarios. Reg. Environ. Chang. 15, 461-473. https://doi.org/10.1007/s10113-014-0606-z

Hulme, M., 1997. The climate in the UK from November 1994 to October 1995. Weather 52, 242257. https://doi.org/10.1002/j.1477-8696.1997.tb06320.x

Hulme, M., Barrow, E., 1997. Climate of the British Isles: present, past and future. Routledge, London.

Hulme, M., Briffa, K.R., Jones, P.D., Senior, C.A., Briffal, K.R., Jones, P.D., Senior, C.A., Briffa, K.R., Jones, P.D., Senior, C.A., 1993. Validation of GCM control simulations using indices of daily airflow types over the British Isles. Clim. Dyn. 9, 95-105. https://doi.org/10.1007/BF00210012

Huntingford, C., Marsh, T., Scaife, A.A., Kendon, E.J., Hannaford, J., Kay, A.L., Lockwood, M., Prudhomme, C., Reynard, N.S., Parry, S., Lowe, J.A., Screen, J.A., Ward, H.C., Roberts, M., Stott, P.A., Bell, V.A., Bailey, M., Jenkins, A., Legg, T., Otto, F.E.L., Massey, N., Schaller, N., Slingo, J., Allen, M.R., 2014. Potential influences on the United Kingdom's floods of winter 2013/14. Nat. Clim. Chang. 4, 769-777. https://doi.org/10.1038/nclimate2314

Hurrell, J.W., 1995. Decadal Trends in the North Atlantic Oscillation: Regional Temperatures and Precipitation. Science (80-. ). 269, 676-679.

Huth, R., 2010. Synoptic-climatological applicability of circulation classifications from the COST733 collection: First results. Phys. Chem. Earth, Parts A/B/C 35, 388-394. https://doi.org/https://doi.org/10.1016/j.pce.2009.11.013

Ikeuchi, H., Hirabayashi, Y., Yamazaki, D., Muis, S., Ward, P.J., Winsemius, H.C., Verlaan, M., Kanae, S., 2017. Compound simulation of fluvial floods and storm surges in a global coupled river-coast flood model: Model development and its application to 2007 Cyclone Sidr in

Bangladesh. J. Adv. Model. Earth Syst. 9, 1847-1862. https://doi.org/10.1002/2017MS000943 IPCC, 2018. Summary for Policymakers. In: Global Warming of $1.5^{\circ} \mathrm{C}$. An IPCC Special Report on the impacts of global warming of $1.5^{\circ} \mathrm{C}$ above pre-industrial levels and related global greenhouse gas emission pathways, in the context of strengthening the global, World Meteorological Organization, Geneva, Switzerland.

IPCC, 2012. Managing the Risks of Extreme Events and Disasters to Advance Climate Change Adaptation. A Special Report of Working Groups I and II of the Intergovernmental Panel on Climate Change. Cambridge University Press, Cambridge, UK, and New York, NY, USA. https://doi.org/10.1017/CBO9781139177245 
Irannezhad, M., Ahmadi, B., Kløve, B., Moradkhani, H., 2017. Atmospheric circulation patterns explaining climatological drought dynamics in the boreal environment of Finland, 1962-2011.

Int. J. Climatol. 37, 801-817. https://doi.org/10.1002/joc.5039

Jenkinson, A.F., Collison, F.P., 1977. An Initial Climatology of Gales over the North Sea. Synoptic Climatology Branch Memorandum No. 62, Meteorological Office, Bracknell.

Johnson, K., Depietri, Y., Breil, M., 2016. Multi-hazard risk assessment of two Hong Kong districts. Int. J. Disaster Risk Reduct. 19, 311-323. https://doi.org/http://dx.doi.org/10.1016/j.ijdrr.2016.08.023

Jones, J.M., Davies, T.D., 2000. The influence of climate on air and precipitation chemistry over Europe and downscaling applications to future acidic deposition. Clim. Res. 14, 7-24. Jones, P.D., Harpham, C., Briffa, K.R., 2013. Lamb weather types derived from reanalysis products. Int. J. Climatol. 33, 1129-1139. https://doi.org/10.1002/joc.3498

Jones, P.D., Harpham, C., Lister, D., 2016. Long-term trends in gale days and storminess for the Falkland Islands. Int. J. Climatol. 36, 1413-1427. https://doi.org/10.1002/joc.4434 Jones, P.D., Hulme, M., Briffa, K.R., 1993. A comparison of Lamb circulation types with an objective classification scheme. Int. J. Climatol. 13, 655-663. https://doi.org/10.1002/joc.3370130606

Jones, P.D., Osborn, T.J., Harpham, C., Briffa, K.R., 2014. The development of Lamb weather types: From subjective analysis of weather charts to objective approaches using reanalyses. Weather 69, 128-132. https://doi.org/10.1002/wea.2255

Jongman, B., Hochrainer-Stigler, S., Feyen, L., Aerts, J.C.J.H., Mechler, R., Botzen, W.J.W., Bouwer, L.M., Pflug, G., Rojas, R., Ward, P.J., 2014. Increasing stress on disaster-risk finance due to large floods. Nat. Clim. Chang. 4, 264-268. https://doi.org/10.1038/nclimate2124 Jonkman, S.N., 2005. Global perspectives on loss of human life caused by floods. Nat. Hazards 34, 151-175. https://doi.org/10.1007/s11069-004-8891-3

Kalkstein, L.S., Nichols, M.C., Barthel, C.D., Greene, J.S., 1996. A new spatial synoptic classification: application to air-mass analysis. Int. J. Climatol. 16, 983-1004. https://doi.org/10.1002/(SICI)1097-0088(199609)16:9<983::AID-JOC61>3.0.CO;2-N Kalnay, E., Kanamitsu, M., Kistler, R., Collins, W., Deaven, D., Gandin, L., Iredell, M., Saha, S., White, G., Woollen, J., Zhu, Y., Leetmaa, A., Reynolds, R., Chelliah, M., Ebisuzaki, W., Higgins, W., Janowiak, J., Mo, K.C., Ropelewski, C., Wang, J., Jenne, R., Joseph, D., 1996. The NCEP/NCAR 40-Year Reanalysis Project. Bull. Am. Meteorol. Soc. 77, 437-471. https://doi.org/https://doi.org/10.1175/1520-0477(1996)077<0437:TNYRP>2.0.CO;2

Kangas, R.S., Brown, T.J., 2007. Characteristics of US drought and pluvials from a high-resolution 
spatial dataset. Int. J. Climatol. 27, 1303-1325. https://doi.org/10.1002/joc.1473

Kappes, M.S., Keiler, M., von Elverfeldt, K., Glade, T., 2012a. Challenges of analyzing multi-hazard risk: a review. Nat. Hazards 64, 1925-1958. https://doi.org/10.1007/s11069-012-0294-2

Kappes, M.S., Papathoma-Köhle, M., Keiler, M., 2012b. Assessing physical vulnerability for multihazards using an indicator-based methodology. Appl. Geogr. 32, 577-590.

https://doi.org/http://dx.doi.org/10.1016/j.apgeog.2011.07.002

Kargel, J.S., Leonard, G.J., Shugar, D.H., Haritashya, U.K., Bevington, A., Fielding, E.J., Fujita, K., Geertsema, M., Miles, E.S., Steiner, J., Anderson, E., Bajracharya, S., Bawden, G.W., Breashears, D.F., Byers, A., Collins, B., Dhital, M.R., Donnellan, A., Evans, T.L., Geai, M.L., Glasscoe, M.T., Green, D., Gurung, D.R., Heijenk, R., Hilborn, A., Hudnut, K., Huyck, C., Immerzeel, W.W., Liming, J., Jibson, R., Kääb, A., Khanal, N.R., Kirschbaum, D., Kraaijenbrink, P.D.A., Lamsal, D., Shiyin, L., Mingyang, L., McKinney, D., Nahirnick, N.K., Zhuotong, N., Ojha, S., Olsenholler, J., Painter, T.H., Pleasants, M., Pratima, K.C., Yuan, Q.I., Raup, B.H., Regmi, D., Rounce, D.R., Sakai, A., Donghui, S., Shea, J.M., Shrestha, A.B., Shukla, A., Stumm, D., van der Kooij, M., Voss, K., Xin, W., Weihs, B., Wolfe, D., Lizong, W., Xiaojun, Y., Yoder, M.R., Young, N., 2016. Geomorphic and geologic controls of geohazards induced by Nepal's 2015 Gorkha earthquake. Science (80-. ). 351.

Kates, R.W., Colten, C.E., Laska, S., Leatherman, S.P., 2006. Reconstruction of New Orleans after Hurricane Katrina: A research perspective. Proc. Natl. Acad. Sci. 103, 14653 LP - 14660. https://doi.org/10.1073/pnas.0605726103

Keef, C., Svensson, C., Tawn, J.A., 2009. Spatial dependence in extreme river flows and precipitation for Great Britain. J. Hydrol. 378, 240-252. https://doi.org/10.1016/j.jhydrol.2009.09.026

Keef, C., Tawn, J.A., Lamb, R., 2013. Estimating the probability of widespread flood events.

Environmetrics 24, 13-21. https://doi.org/10.1002/env.2190

Kelman, I., 2001. The autumn 2000 floods in England and flood management. Weather 56, 346-348. https://doi.org/10.1002/j.1477-8696.2001.tb06507.x

Kendall, M., 1975. Multivariate analysis. Griffin, London.

Kendon, M., 2015. Editorial: The UK storms of winter 2013/2014. Weather 70, 39-40.

https://doi.org/10.1002/wea.2474

Kendon, M., Marsh, T., Parry, S., 2013. The 2010-2012 drought in England and Wales. Weather 68, 88-95. https://doi.org/10.1002/wea.2101

Kendon, M., McCarthy, M., 2015. The UK's wet and stormy winter of 2013/2014. Weather 70, 4047. https://doi.org/10.1002/wea.2465 
Khaliq, M.N., Gachon, P., 2010. Pacific Decadal Oscillation Climate Variability and Temporal Pattern of Winter Flows in Northwestern North America. J. Hydrometeorol. 11, 917-933. https://doi.org/10.1175/2010JHM1254.1

Kiem, A.S., Franks, S.W., Kuczera, G., 2003. Multi-decadal variability of flood risk. Geophys. Res. Lett. 30. https://doi.org/10.1029/2002GL015992

Kim, S., 2015. ppcor: An R Package for a Fast Calculation to Semi-partial Correlation Coefficients. Commun. Stat. Appl. methods 22, 665-674. https://doi.org/10.5351/CSAM.2015.22.6.665 Kjeldsen, T.R., Jones, D.A., 2009. A formal statistical model for pooled analysis of extreme floods. Hydrol. Res. 40, 465-480.

Knight, J., Maidens, A., Watson, P., Andrews, M., Belcher, S., Brunet, G., Fereday, D., Folland, C., Scaife, A., Slingo, J., 2017. Global meteorological influences on the record UK rainfall of winter 2013-14. Environ. Res. Lett. 12. https://doi.org/10.1088/1748-9326/aa693c

Knutson, T.R., McBride, J.L., Chan, J., Emanuel, K., Holland, G., Landsea, C., Held, I., Kossin, J.P., Srivastava, A.K., Sugi, M., 2010. Tropical cyclones and climate change. Nat. Geosci. 3, 157. Koks, E.E., Rozenberg, J., Zorn, C., Tariverdi, M., Vousdoukas, M., Fraser, S.A., Hall, J.W., Hallegatte, S., 2019. A global multi-hazard risk analysis of road and railway infrastructure assets. Nat. Commun. 10, 2677. https://doi.org/10.1038/s41467-019-10442-3

Kreibich, H., Blauhut, V., Aerts, J.C.J.H., Bouwer, L.M., Van Lanen, H.A.J., Mejia, A., Mens, M., Van Loon, A.F., 2019. How to improve attribution of changes in drought and flood impacts AU - Kreibich, Heidi. Hydrol. Sci. J. 1-18. https://doi.org/10.1080/02626667.2018.1558367 Kunte, P.D., Jauhari, N., Mehrotra, U., Kotha, M., Hursthouse, A.S., Gagnon, A.S., 2014. Multihazards coastal vulnerability assessment of Goa, India, using geospatial techniques. Ocean Coast. Manag. 95, 264-281. https://doi.org/http://dx.doi.org/10.1016/j.ocecoaman.2014.04.024 Lamb, H.H., 1972. British Isles weather types and a register of the daily sequence of circulation patterns, Geophysical Memoirs. London: HMSO.

Lamb, H.H., 1950. Types and spells of weather around the year in the British Isles : Annual trends, seasonal structure of the year, singularities. Q. J. R. Meteorol. Soc. 76, 393-429. https://doi.org/10.1002/qj.49707633005

Lamb, R., 2006. Rainfall-runoff modelling for flood frequency estimation. Encycl. Hydrol. Sci. $11: 125$.

Lamb, R., Keef, C., Tawn, J., Laeger, S., Meadowcroft, I., Surendran, S., Dunning, P., Batstone, C., 2010. A new method to assess the risk of local and widespread flooding on rivers and coasts. J. Flood Risk Manag. 3, 323-336. https://doi.org/10.1111/j.1753-318X.2010.01081.x Larkin, N.K., Harrison, D.E., 2005. Global seasonal temperature and precipitation anomalies during 
El Niño autumn and winter. Geophys. Res. Lett. 32. https://doi.org/10.1029/2005GL022860 Lavers, D., Allan, R.P., Villarini, G., Lloyd-Hughes, B., Brayshaw, D.J., Wade, A.J., 2013. Future changes in atmospheric rivers and their implications for winter flooding in Britain. Environ.

Res. Lett. 8, 34010. https://doi.org/10.1088/1748-9326/8/3/034010

Lavers, D.A., Allan, R.P., Wood, E.F., Villarini, G., Brayshaw, D.J., Wade, A.J., 2011. Winter floods in Britain are connected to atmospheric rivers. Geophys. Res. Lett. 38, 1-8. https://doi.org/10.1029/2011GL049783

Le Tertre, A., Lefranc, A., Eilstein, D., Declercq, C., Medina, S., Blanchard, M., Chardon, B., Fabre, P., Filleul, L., Jusot, J.-F., Pascal, L., Prouvost, H., Cassadou, S., Ledrans, M., 2006. Impact of the 2003 Heatwave on All-Cause Mortality in 9 French Cities. Epidemiology 17, 75-79.

Lee, D., Ward, P., Block, P., 2018. Attribution of Large-Scale Climate Patterns to Seasonal PeakFlow and Prospects for Prediction Globally. Water Resour. Res. 54, 916-938. https://doi.org/10.1002/2017WR021205

Lenderink, G., Fowler, H.J., 2017. Understanding rainfall extremes. Nat. Clim. Chang. 7, 391. Leng, G., Hall, J., 2019. Crop yield sensitivity of global major agricultural countries to droughts and the projected changes in the future. Sci. Total Environ. 654, 811-821. https://doi.org/https://doi.org/10.1016/j.scitotenv.2018.10.434

Leonard, M., Westra, S., Phatak, A., Lambert, M., van den Hurk, B., McInnes, K., Risbey, J., Schuster, S., Jakob, D., Stafford-Smith, M., 2014. A compound event framework for understanding extreme impacts. Wiley Interdiscip. Rev. Clim. Chang. 5, 113-128. https://doi.org/10.1002/wcc.252

Levinson, D.H., Waple, A.M., 2004. STATE OF THE CLIMATE IN 2003. Bull. Am. Meteorol. Soc. $85, \mathrm{~S} 1-\mathrm{S} 72$.

Liu, B., Siu, Y.L., Mitchell, G., 2017. A quantitative model for estimating risk from multiple interacting natural hazards: an application to northeast Zhejiang, China. Stoch. Environ. Res.

Risk Assess. 31, 1319-1340. https://doi.org/10.1007/s00477-016-1250-6

Liu, C., Allan, R.P., 2013. Observed and simulated precipitation responses in wet and dry regions 1850-2100. Environ. Res. Lett. 8, 34002. https://doi.org/10.1088/1748-9326/8/3/034002

Liu, Z., Tang, Y., Jian, Z., Poulsen, C.J., Welker, J.M., Bowen, G.J., 2017. Pacific North American circulation pattern links external forcing and North American hydroclimatic change over the past millennium. Proc. Natl. Acad. Sci. 114, 3340-3345.

https://doi.org/10.1073/pnas.1618201114

Lorenzo, M.N., Ramos, A.M., Taboada, J.J., Gimeno, L., 2011. Changes in Present and Future Circulation Types Frequency in Northwest Iberian Peninsula. PLoS One 6, e16201. 
https://doi.org/10.1371/journal.pone.0016201

Lorenzo, M.N., Taboada, J.J., Gimeno, L., 2008. Links between circulation weather types and teleconnection patterns and their influence on precipitation patterns in Galicia (NW Spain). Int.

J. Climatol. 28, 1493-1505. https://doi.org/10.1002/joc.1646

Lucarini, V., Faranda, D., Freitas, J.M., Holland, M., Kuna, T., Nicol, M., Vaienti, S., 2016.

Extremes and recurrence in dynamical systems. John Wiley \& Sons.

Lucarini, V., Faranda, D., Wouters, J., 2012. Universal Behaviour of Extreme Value Statistics for

Selected Observables of Dynamical Systems. J. Stat. Phys. 147, 63-73. https://doi.org/10.1007/s10955-012-0468-z

Ludwig, P., Schaffernicht, E.J., Shao, Y., Pinto, J.G., 2016. Regional atmospheric circulation over Europe during the Last Glacial Maximum and its links to precipitation. J. Geophys. Res. Atmos. 121, 2130-2145. https://doi.org/10.1002/2015JD024444

Luo, J.-J., Liu, G., Hendon, H., Alves, O., Yamagata, T., 2017. Inter-basin sources for two-year predictability of the multi-year La Niña event in 2010-2012. Sci. Rep. 7, 2276. https://doi.org/10.1038/s41598-017-01479-9

Mahendra, R.S., Mohanty, P.C., Bisoyi, H., Kumar, T.S., Nayak, S., 2011. Assessment and management of coastal multi-hazard vulnerability along the Cuddalore-Villupuram, east coast of India using geospatial techniques. Ocean Coast. Manag. 54, 302-311. https://doi.org/http://dx.doi.org/10.1016/j.ocecoaman.2010.12.008

Mallakpour, I., Villarini, G., 2016. Investigating the relationship between the frequency of flooding over the central United States and large-scale climate. Adv. Water Resour. 92, 159-171. https://doi.org/https://doi.org/10.1016/j.advwatres.2016.04.008

Mallakpour, I., Villarini, G., 2015. The changing nature of flooding across the central United States.

Nat. Clim. Chang. 5, 250-254. https://doi.org/10.1038/nclimate2516

Mann, H.B., 1945. Nonparametric Tests Against Trend. Econometrica 13, 245-259. https://doi.org/10.2307/1907187

Mann, H.B., Whitney, D.R., 1947. On a Test of Whether one of Two Random Variables is Stochastically Larger than the Other. Ann. Math. Stat. 18, 50-60. https://doi.org/10.1214/aoms/1177730491

Mantua, N.J., Hare, S.R., 2002. The Pacific Decadal Oscillation. J. Oceanogr. 58, 35-44. https://doi.org/10.1023/A:1015820616384

Marsh, T., 2008. A hydrological overview of the summer 2007 floods in England and Wales. Weather 63.

Marsh, T., 2004. The January 2003 flood on the Thames. Weather 59, 59-62. 
https://doi.org/10.1256/wea.212.03

Marsh, T., Dale, M., 2002. The UK Floods of 2000-2001: A Hydrometeorological Appraisal. Water Environ. J. 16, 180-188. https://doi.org/10.1111/j.1747-6593.2002.tb00392.x

Marsh, T., Hannaford, J., 2007. The summer 2007 floods in England \& Wales - a hydrological appraisal. Cent. Ecol. Hydrol. 32.

Martin, E.R., 2018. Future Projections of Global Pluvial and Drought Event Characteristics.

Geophys. Res. Lett. 45, 11,911-913,920. https://doi.org/10.1029/2018GL079807

Martius, O., Pfahl, S., Chevalier, C., 2016. A global quantification of compound precipitation and wind extremes. Geophys. Res. Lett. 43, 7709-7717. https://doi.org/10.1002/2016GL070017

Marvel, K., Cook, B.I., Bonfils, C.J.W., Durack, P.J., Smerdon, J.E., Williams, A.P., 2019.

Twentieth-century hydroclimate changes consistent with human influence. Nature 569, 59-65. https://doi.org/10.1038/s41586-019-1149-8

Matthews, T., Mullan, D., Wilby, R.L., Broderick, C., Murphy, C., 2016a. Past and future climate change in the context of memorable seasonal extremes. Clim. Risk Manag. 11, 37-52. https://doi.org/https://doi.org/10.1016/j.crm.2016.01.004

Matthews, T., Murphy, C., McCarthy, G., Broderick, C., Wilby, R.L., 2018. Super Storm Desmond: a process-based assessment. Environ. Res. Lett. 13, 14024.

Matthews, T., Murphy, C., Wilby, R.L., Harrigan, S., 2016b. A cyclone climatology of the BritishIrish Isles 1871-2012. Int. J. Climatol. 36, 1299-1312. https://doi.org/10.1002/joc.4425

Matthews, T., Murphy, C., Wilby, R.L., Harrigan, S., 2014. Stormiest winter on record for Ireland and UK. Nature 4, 738-740. https://doi.org/10.1038/nclimate2336

Matthews, T.K.R., Wilby, R.L., Murphy, C., 2017. Communicating the deadly consequences of global warming for human heat stress. Proc. Natl. Acad. Sci. 201617526.

https://doi.org/10.1073/pnas.1617526114

McAlpine, C.A., Syktus, J., Deo, R.C., Lawrence, P.J., McGowan, H.A., Watterson, I.G., Phinn,

S.R., 2007. Modeling the impact of historical land cover change on Australia's regional climate. Geophys. Res. Lett. 34. https://doi.org/10.1029/2007GL031524

McCarthy, M., Spillane, S., Walsh, S., Kendon, M., 2016. The meteorology of the exceptional winter of 2015/2016 across the UK and Ireland. Weather 71, 305-313.

https://doi.org/10.1002/wea.2823

McKee T.B., Doesken N.J., K.J., 1995. Drought monitoring with multiple time scales. Proc. 9th Conf. Appl. Climatol. 233-236. https://doi.org/10.1007/s13398-014-0173-7.2

McKee, T.B., Doesken, N.J., Kleist, J., 1993. The relationship of drought frequency and duration to time scales. AMS 8th Conf. Appl. Climatol. 179-184. https://doi.org/citeulike-article- 
id:10490403

Merz, R. Blöschl, G., 2003. A process typology of regional floods. Water Resour. Res. 39, SWC 5: 1-20. https://doi.org/10.1029/2002WR001952

Merz, B., Aerts, J., Arnbjerg-Nielsen, K., Baldi, M., Becker, A., Bichet, A., Blöschl, G., Bouwer, L.M., Brauer, A., Cioffi, F., Delgado, J.M., Gocht, M., Guzzetti, F., Harrigan, S., Hirschboeck, K., Kilsby, C., Kron, W., Kwon, H.H., Lall, U., Merz, R., Nissen, K., Salvatti, P., Swierczynski, T., Ulbrich, U., Viglione, A., Ward, P.J., Weiler, M., Wilhelm, B., Nied, M., 2014. Floods and climate: Emerging perspectives for flood risk assessment and management. Nat. Hazards Earth Syst. Sci. 14, 1921-1942. https://doi.org/10.5194/nhess-14-1921-2014

Merz, B., Kreibich, H., Schwarze, R., Thieken, A., 2010. Review article “Assessment of economic flood damage.” Nat. Hazards Earth Syst. Sci. 10, 1697-1724. https://doi.org/10.5194/nhess-10$1697-2010$

Messori, G., Caballero, R., Faranda, D., 2017. A dynamical systems approach to studying midlatitude weather extremes. Geophys. Res. Lett. 44, 3346-3354.

https://doi.org/10.1002/2017GL072879

Met Office, n.d. UK storm season 2015/16 [WWW Document].

Met Office, n.d. UK storm season 2016/17 [WWW Document].

Met Office, n.d. UK storm season 2017/18 [WWW Document]. Meteorological Office, 1992. Monthly Weather Report, November 1992.

Milly, P.C.D., Wetherald, R.T., Dunne, K.A., Delworth, T.L., 2002. Increasing risk of great floods in a changing climate. Nature 415, 514-517. https://doi.org/10.1038/415514a

Min, S.-K., Zhang, X., Zwiers, F.W., Hegerl, G.C., 2011. Human contribution to more-intense precipitation extremes. Nature 470, 378.

Muchan, K., Lewis, M., Hannaford, J., Parry, S., 2015. The winter storms of 2013/2014 in the UK: hydrological responses and impacts. Weather 70, 55-61. https://doi.org/10.1002/wea.2469

Munich Re, 2019. NatCatSERVICE - Natural catastrophes in 2018.

Munich Re, 2017a. NatCatSERVICE Analysis Tool.

Munich Re, 2017b. Topics Geo Natural Catastraphes.

Munich Re, 2015. Natural catastrophes 2014: Analyses, assessments, positions, Topics Geo.

Munoz, S.E., Giosan, L., Therrell, M.D., Remo, J.W.F., Shen, Z., Sullivan, R.M., Wiman, C., O’Donnell, M., Donnelly, J.P., 2018. Climatic control of Mississippi River flood hazard amplified by river engineering. Nature 556, 95.

Murawski, A., Bürger, G., Vorogushyn, S., Merz, B., 2016. Can local climate variability be explained by weather patterns? A multi-station evaluation for the Rhine basin. Hydrol. Earth 
Syst. Sci. 20, 4283-4306. https://doi.org/10.5194/hess-20-4283-2016

Murawski, A., Vorogushyn, S., Bürger, G., Gerlitz, L., Merz, B., 2018. Do Changing Weather Types Explain Observed Climatic Trends in the Rhine Basin? An Analysis of Within- and BetweenType Changes. J. Geophys. Res. Atmos. 123, 1562-1584. https://doi.org/10.1002/2017JD026654

Murphy, J.M., Sexton, D.M.H., Jenkins, G.J., Boorman, P.M., Booth, B.B.B., Brown, C.C., Clark, R.T., Collins, M., Harris, G.R., Kendon, E.J., Betts, R.A., Brown, S.J., Howard, T.P., Humphrey, K.A., McCarthy, M.P., McDonald, R.E., Stephens, A., Wallace, C., Warren, R., Wilby, R., Wood, R.A., 2009. UK Climate Projections Science Report: Climate change projections. Exeter.

Naumann, G., Spinoni, J., Vogt, J. V, Barbosa, P., 2015. Assessment of drought damages and their uncertainties in Europe. Environ. Res. Lett. 10, 124013.

Neal, E.G., Todd Walter, M., Coffeen, C., 2002. Linking the pacific decadal oscillation to seasonal stream discharge patterns in Southeast Alaska. J. Hydrol. 263, 188-197. https://doi.org/https://doi.org/10.1016/S0022-1694(02)00058-6

Neal, R.A., Phillips, I.D., 2011. Winter daily precipitation variability over Cumbria, Northwest England. Theor. Appl. Climatol. 106, 245. https://doi.org/10.1007/s00704-011-0483-z

Ng, J.Y., Turner, S.W.D., Galelli, S., 2017. Influence of El Niño Southern Oscillation on global hydropower production. Environ. Res. Lett. 12, 34010. https://doi.org/10.1088/1748-

\section{6/aa5ef8}

Nied, M., Schröter, K., Lüdtke, S., Dung Nguyen, V., Merz, B., 2016. What are the hydrometeorological controls on flood characteristics? J. Hydrol. 545, 310-326.

https://doi.org/10.1016/j.jhydrol.2016.12.003

Ning, L., Bradley, R.S., 2016. NAO and PNA influences on winter temperature and precipitation over the eastern United States in CMIP5 GCMs. Clim. Dyn. 46, 1257-1276. https://doi.org/10.1007/s00382-015-2643-9

NOAA, 2011. State of the Climate: Drought for December 2010. https://www.ncdc.noaa.gov/sotc/drought/201012.

Nobre, G.G., Jongman, B., Aerts, J., Ward, P.J., 2017. The role of climate variability in extreme floods in Europe. Environ. Res. Lett. 12, 84012.

NWRFC, 2010. 2010 Northwest Floods. https://www.nwrfc.noaa.gov/floods/dec_2010/2010_Northwest_Flood.pdf 19.

O’Hare, G.P.P., Wilby, R.L., 1995. A Review of Ozone Pollution in the United Kingdom and Ireland with an Analysis Using Lamb Weather Types. Geogr. J. 161, 1-20. 
https://doi.org/10.2307/3059923

Oni, S., Futter, M., Ledesma, J., Teutschbein, C., Buttle, J., Laudon, H., 2016. Using dry and wet year hydroclimatic extremes to guide future hydrologic projections. Hydrol. Earth Syst. Sci. 20, 2811-2825. https://doi.org/10.5194/hess-20-2811-2016

Oouchi, K., Yoshimura, J., Yoshimura, H., Mizuta, R., Kusunoki, S., Noda, A., 2006. Tropical Cyclone Climatology in a Global-Warming Climate as Simulated in a $20 \mathrm{~km}-\mathrm{Mesh}$ Global Atmospheric Model: Frequency and Wind Intensity Analyses. J. Meteorol. Soc. Japan. Ser. II 84, 259-276. https://doi.org/10.2151/jmsj.84.259

Otero, N., Sillmann, J., Butler, T., 2018. Assessment of an extended version of the JenkinsonCollison classification on CMIP5 models over Europe. Clim. Dyn. 50, 1559-1579. https://doi.org/10.1007/s00382-017-3705-y

Ouyang, R., Liu, W., Fu, G., Liu, C., Hu, L., Wang, H., 2014. Linkages between ENSO/PDO signals and precipitation, streamflow in China during the last 100 years. Hydrol. Earth Syst. Sci. 18, 3651-3661. https://doi.org/10.5194/hess-18-3651-2014

Pall, P., Aina, T., Stone, D. a, Stott, P. a, Nozawa, T., Hilberts, A.G.J., Lohmann, D., Allen, M.R., 2011. Anthropogenic greenhouse gas contribution to flood risk in England and Wales in autumn 2000. Nature 470, 382-385. https://doi.org/10.1038/nature09762

Palmer, W., 1965. Meteorological Drought. U.S. Res. Pap. No. 45. US Weather Bur. DC. Paredes, D., Trigo, R.M., Garcia-Herrera, R., Trigo, I.F., 2006. Understanding Precipitation Changes in Iberia in Early Spring: Weather Typing and Storm-Tracking Approaches. J. Hydrometeorol. 7, 101-113. https://doi.org/10.1175/JHM472.1

Parry, S., Marsh, T., Kendon, M., 2013. 2012: From drought to floods in England and Wales. Weather 68, 268-274. https://doi.org/10.1002/wea.2152

Pattison, I., Lane, S.N., 2012. The relationship between Lamb weather types and long-term changes in flood frequency, River Eden, UK. Int. J. Climatol. 32, 1971-1989. https://doi.org/10.1002/joc.2415

Pechlivanidis, I.G., Arheimer, B., Donnelly, C., Hundecha, Y., Huang, S., Aich, V., Samaniego, L., Eisner, S., Shi, P., 2017. Analysis of hydrological extremes at different hydro-climatic regimes under present and future conditions. Clim. Change 141, 467-481. https://doi.org/10.1007/s10584-016-1723-0

Peña-Angulo, D., Trigo, R.M., Cortesi, N., González-Hidalgo, J.C., 2016. The influence of weather types on the monthly average maximum and minimum temperatures in the Iberian Peninsula. Atmos. Res. 178-179, 217-230. https://doi.org/https://doi.org/10.1016/j.atmosres.2016.03.022 Perry, A.H., 1980. A NOTE ON THE SOUTH WALES FLOODS OF LATE DECEMBER 1979. 
Weather 35, 106-109. https://doi.org/10.1002/j.1477-8696.1980.tb03506.x

Perry, R.W., Lindell, M.K., 2008. Volcanic risk perception and adjustment in a multi-hazard environment. J. Volcanol. Geotherm. Res. 172, 170-178. https://doi.org/http://dx.doi.org/10.1016/j.jvolgeores.2007.12.006

Pfahl, S., O’Gorman, P.A., Fischer, E.M., 2017. Understanding the regional pattern of projected future changes in extreme precipitation. Nat. Clim. Chang. 7, 423.

Pielke, R.A., Gratz, J., Landsea, C.W., Collins, D., Saunders, M.A., Musulin, R., 2008. Normalized Hurricane Damage in the United States: 1900-2005. Nat. Hazards Rev. 9, 29-42. https://doi.org/10.1061/(ASCE)1527-6988(2008)9:1(29)

Pinto, J.G., Zacharias, S., Fink, A.H., Leckebusch, G.C., Ulbrich, U., 2009. Factors contributing to the development of extreme North Atlantic cyclones and their relationship with the NAO. Clim. Dyn. 32, 711-737. https://doi.org/10.1007/s00382-008-0396-4

Plavcová, E., Kyselý, J., Štěpánek, P., 2014. Links between circulation types and precipitation in Central Europe in the observed data and regional climate model simulations. Int. J. Climatol.

34, 2885-2898. https://doi.org/10.1002/joc.3882

Pleijel, H., Grundström, M., Karlsson, G.P., Karlsson, P.E., Chen, D., 2016. A method to assess the inter-annual weather-dependent variability in air pollution concentration and deposition based on weather typing. Atmos. Environ. 126, 200-210. https://doi.org/https://doi.org/10.1016/j.atmosenv.2015.11.053

Pope, R.J., Butt, E.W., Chipperfield, M.P., Doherty, R.M., Fenech, S., Schmidt, A., Arnold, S.R., Savage, N.H., 2016. The impact of synoptic weather on UK surface ozone and implications for premature mortality. Environ. Res. Lett. 11. https://doi.org/10.1088/1748-9326/11/12/124004 Pope, R.J., Savage, N.H., Chipperfield, M.P., Arnold, S.R., Osborn, T.J., 2014. The influence of synoptic weather regimes on UK air quality: analysis of satellite column $\mathrm{NO}$ 2. Atmos. Sci. Lett. 15, 211-217. https://doi.org/10.1002/as12.492

Pope, R.J., Savage, N.H., Chipperfield, M.P., Ordonez, C., Neal, L.S., 2015. The influence of synoptic weather regimes on UK air quality: Regional model studies of tropospheric column NO2. Atmos. Chem. Phys. 15, 11201-11215. https://doi.org/10.5194/acp-15-11201-2015 Prein, A.F., Bukovsky, M.S., Mearns, L.O., Bruyère, C.L., Done, J.M., 2019. Simulating North American Weather Types With Regional Climate Models. Front. Environ. Sci.

Priestley, M.D.K., Pinto, J.G., Dacre, H.F., Shaffrey, L.C., 2017. The role of cyclone clustering during the stormy winter of 2013/2014. Weather 72, 187-192. https://doi.org/10.1002/wea.3025 Pritchard, O.G., Hallett, S.H., Farewell, T.S., 2015. Probabilistic soil moisture projections to assess Great Britain's future clay-related subsidence hazard. Clim. Change 133, 635-650. 
https://doi.org/10.1007/s10584-015-1486-Z

Prudhomme, C., Giuntoli, I., Robinson, E.L., Clark, D.B., Arnell, N.W., Dankers, R., Fekete, B.M., Franssen, W., Gerten, D., Gosling, S.N., Hagemann, S., Hannah, D.M., Kim, H., Masaki, Y., Satoh, Y., Stacke, T., Wada, Y., Wisser, D., 2014. Hydrological droughts in the 21st century, hotspots and uncertainties from a global multimodel ensemble experiment. Proc. Natl. Acad.

Sci. 111, 3262 LP - 3267. https://doi.org/10.1073/pnas.1222473110

Qu Y.; Dodov B.; Jain V.; Hautaniemi T., 2010. An inland flood estimation model for Great Britain. Manag. Consequences a Chang. Glob. Environ.

Quesada-Montano, B., Di Baldassarre, G., Rangecroft, S., Van Loon, A.F., 2018. Hydrological change: Towards a consistent approach to assess changes on both floods and droughts. Adv. Water Resour. 111, 31-35. https://doi.org/https://doi.org/10.1016/j.advwatres.2017.10.038

Rahmstorf, S., Coumou, D., 2011. Increase of extreme events in a warming world. Proc. Natl. Acad. Sci. 108, 17905 LP - 17909. https://doi.org/10.1073/pnas.1101766108

Ramos, A.M., Lorenzo, M.N., Gimeno, L., 2010. Compatibility between modes of low-frequency variability and circulation types: A case study of the northwest Iberian Peninsula. J. Geophys.

Res. Atmos. 115. https://doi.org/10.1029/2009JD012194

Rayner, N.A., Parker, D.E., Horton, E.B., Folland, C.K., Alexander, L. V, Rowell, D.P., Kent, E.C., Kaplan, A., 2003. Global analyses of sea surface temperature, sea ice, and night marine air temperature since the late nineteenth century. J. Geophys. Res. Atmos. 108.

https://doi.org/10.1029/2002JD002670

Reliefweb, 2010. India: Floods - Dec 2010. https://reliefweb.int/disaster/fl-2010-000249-ind. Reliefweb, 2003. Madagascar: Floods - Jan 2003. https://reliefweb.int/disaster/fl-2003-0037-mdg.

Richardson, D., Fowler, H.J., Kilsby, C.G., Neal, R., 2018. A new precipitation and drought climatology based on weather patterns. Int. J. Climatol. 38, 630-648.

https://doi.org/10.1002/joc.5199

Rimbu, N., Dima, M., Lohmann, G., Stefan, S., 2004. Impacts of the North Atlantic Oscillation and the El Niño-Southern Oscillation on Danube river flow variability. Geophys. Res. Lett. 31.

https://doi.org/10.1029/2004GL020559

Rodrigues, D., Alvarez-Castro, M.C., Messori, G., Yiou, P., Robin, Y., Faranda, D., 2018.

Dynamical Properties of the North Atlantic Atmospheric Circulation in the Past 150 Years in

CMIP5 Models and the 20CRv2c Reanalysis. J. Clim. 31, 6097-6111.

https://doi.org/10.1175/JCLI-D-17-0176.1

Rogers, J.C., Coleman, J.S.M., 2003. Interactions between the Atlantic Multidecadal Oscillation, El Niño/La Niña, and the PNA in winter Mississippi Valley stream flow. Geophys. Res. Lett. 30. 
https://doi.org/10.1029/2003GL017216

Rosendahl Appelquist, L., Halsnæs, K., 2015. The Coastal Hazard Wheel system for coastal multihazard assessment \& management in a changing climate. J. Coast. Conserv. 19, 157-179. https://doi.org/10.1007/s11852-015-0379-7

Roudier, P., Andersson, J.C.M., Donnelly, C., Feyen, L., Greuell, W., Ludwig, F., 2016. Projections of future floods and hydrological droughts in Europe under a $+2{ }^{\circ} \mathrm{C}$ global warming. Clim.

Change 135, 341-355. https://doi.org/10.1007/s10584-015-1570-4

Rubel, F., Kottek, M., 2010. Observed and projected climate shifts 1901-2100 depicted by world maps of the Köppen-Geiger climate classification. Meteorol. Zeitschrift 19, 135-141.

https://doi.org/10.1127/0941-2948/2010/0430

Russo, S., Sillmann, J., Fischer, E.M., 2015. Top ten European heatwaves since 1950 and their occurrence in the coming decades. Environ. Res. Lett. 10, 124003.

https://doi.org/10.1088/1748-9326/10/12/124003

Sacks, W.J., Deryng, D., Foley, J.A., Ramankutty, N., 2010. Crop planting dates: an analysis of global patterns. Glob. Ecol. Biogeogr. 19, 607-620. https://doi.org/10.1111/j.1466-

8238.2010.00551.x

Sampson, C.C., Fewtrell, T.J., O’Loughlin, F., Pappenberger, F., Bates, P.B., Freer, J.E., Cloke,

H.L., 2014. The impact of uncertain precipitation data on insurance loss estimates using a flood catastrophe model. Hydrol. Earth Syst. Sci. 18, 2305-2324. https://doi.org/10.5194/hess-182305-2014

Sandri, L., Thouret, J.-C., Constantinescu, R., Biass, S., Tonini, R., 2014. Long-term multi-hazard assessment for El Misti volcano (Peru). Bull. Volcanol. 76, 771. https://doi.org/10.1007/s00445013-0771-9

Schaller, N., Kay, A.L., Lamb, R., Massey, N.R., van Oldenborgh, G.J., Otto, F.E.L., Sparrow, S.N., Vautard, R., Yiou, P., Ashpole, I., Bowery, A., Crooks, S.M., Haustein, K., Huntingford, C., Ingram, W.J., Jones, R.G., Legg, T., Miller, J., Skeggs, J., Wallom, D., Weisheimer, A., Wilson, S., Stott, P.A., Allen, M.R., 2016. Human influence on climate in the 2014 southern England winter floods and their impacts. Nat. Clim. Chang. 6, 627-634.

https://doi.org/10.1038/nclimate2927

Schlesinger, M.E., Ramankutty, N., 1994. An oscillation in the global climate system of period 6570 years. Nature 367, 723-726. https://doi.org/10.1038/367723a0

Screen, J.A., Simmonds, I., 2010. The central role of diminishing sea ice in recent Arctic temperature amplification. Nature 464, 1334.

Sedgwick, P., 2014. Multiple hypothesis testing and Bonferroni's correction. BMJ Br. Med. J. 349, 
g6284. https://doi.org/10.1136/bmj.g6284

Seiler, R.A., Hayes, M., Bressan, L., 2002. Using the standardized precipitation index for flood risk monitoring. Int. J. Climatol. 22, 1365-1376. https://doi.org/10.1002/joc.799

Sen, P.K., 1968. Estimates of the Regression Coefficient Based on Kendall's Tau. J. Am. Stat.

Assoc. 63, 1379-1389. https://doi.org/10.1080/01621459.1968.10480934

Sheffield, J., Wood, E.F., Roderick, M.L., 2012. Little change in global drought over the past 60 years. Nature 491, 435. https://doi.org/10.1038/nature11575

Siegert, F., Ruecker, G., Hinrichs, A., Hoffmann, A.A., 2001. Increased damage from fires in logged forests during droughts caused by El Niño. Nature 414, 437-440.

https://doi.org/10.1038/35106547

Sillmann, J., Thorarinsdottir, T., Keenlyside, N., Schaller, N., Alexander, L. V, Hegerl, G.,

Seneviratne, S.I., Vautard, R., Zhang, X., Zwiers, F.W., 2017. Understanding, modeling and predicting weather and climate extremes: Challenges and opportunities. Weather Clim. Extrem.

18, 65-74. https://doi.org/https://doi.org/10.1016/j.wace.2017.10.003

Sinha, D., Syed, T.H., Famiglietti, J.S., Reager, J.T., Thomas, R.C., 2016. Characterizing Drought in India Using GRACE Observations of Terrestrial Water Storage Deficit. J. Hydrometeorol. 18, 381-396. https://doi.org/10.1175/JHM-D-16-0047.1

Slater, L.J., Villarini, G., 2016. Recent trends in U.S. flood risk. Geophys. Res. Lett. 43, 12,428-

12,436. https://doi.org/10.1002/2016GL071199

Smith, J.K.G., Young, M.M., Wilson, K.L., Craig, S.B., 2013. Leptospirosis following a major flood in Central Queensland, Australia. Epidemiol. Infect. 141, 585-590.

Sobel, A.H., Camargo, S.J., Hall, T.M., Lee, C.-Y., Tippett, M.K., Wing, A.A., 2016. Human influence on tropical cyclone intensity. Science (80-. ). 353, 242 LP - 246.

https://doi.org/10.1126/science.aaf6574

Spedicato, G.A., 2017. Discrete Time Markov Chains with R. R J. 9, 84-104.

Stott, P.A., Stone, D.A., Allen, M.R., 2004. Human contribution to the European heatwave of 2003. Nature 432, 610-614. https://doi.org/10.1038/nature03089

Stryhal, J., Huth, R., 2018. Trends in winter circulation over the British Isles and central Europe in twenty-first century projections by 25 CMIP5 GCMs. Clim. Dyn. 0, 0. https://doi.org/10.1007/s00382-018-4178-3

Suleimani, E., Hansen, R., Haeussler, P.J., 2009. Numerical Study of Tsunami Generated by Multiple Submarine Slope Failures in Resurrection Bay, Alaska, during the MW 9.2 1964

Earthquake. Pure Appl. Geophys. 166, 131-152. https://doi.org/10.1007/s00024-004-0430-3 Sun, Q., Miao, C., AghaKouchak, A., Duan, Q., 2016. Century-scale causal relationships between 
global dry/wet conditions and the state of the Pacific and Atlantic Oceans. Geophys. Res. Lett. 43, 6528-6537. https://doi.org/10.1002/2016GL069628

Sun, Y., Zhang, X., Zwiers, F.W., Song, L., Wan, H., Hu, T., Yin, H., Ren, G., 2014. Rapid increase in the risk of extreme summer heat in Eastern China. Nat. Clim. Chang. 4, 1082.

Sutton, R.T., Hodson, D.L.R., 2005. Atlantic Ocean Forcing of North American and European Summer Climate. Science (80-. ). 309, 115-118. https://doi.org/10.1126/science.1109496

Tang, L., Chen, D., Karlsson, P., Gu, Y., Ou, T., 2009. Synoptic circulation and its influence on spring and summer surface ozone concentrations in southern Sweden. Boreal Environ. Res. 14, 889-902.

Tang, Q., Zhang, X., Francis, J.A., 2013. Extreme summer weather in northern mid-latitudes linked to a vanishing cryosphere. Nat. Clim. Chang. 4, 45 .

Tanguy M., Kral F., Fry M., Svensson C., H.J., 2015. Standardised Precipitation Index time series for Integrated Hydrological Units Hydrometric Areas (1961-2012). NERC Environ. Inf. Data Cent.

Taylor, K.E., Stouffer, R.J., Meehl, G.A., 2011. An Overview of CMIP5 and the Experiment Design. Bull. Am. Meteorol. Soc. 93, 485-498. https://doi.org/10.1175/BAMS-D-11-00094.1

Telegraph, 2010. Floods devastate Colombia and Venezuela.

https://www.telegraph.co.uk/news/worldnews/southamerica/colombia/8186408/Floodsdevastate-Colombia-and-Venezuela.html.

Terzi, S., Torresan, S., Schneiderbauer, S., Critto, A., Zebisch, M., Marcomini, A., 2019. Multi-risk assessment in mountain regions: A review of modelling approaches for climate change adaptation. J. Environ. Manage. 232, 759-771. https://doi.org/https://doi.org/10.1016/j.jenvman.2018.11.100

Thierry, P., Stieltjes, L., Kouokam, E., Nguéya, P., Salley, P.M., 2008. Multi-hazard risk mapping and assessment on an active volcano: the GRINP project at Mount Cameroon. Nat. Hazards 45, 429-456. https://doi.org/10.1007/s11069-007-9177-3

Tilloy, A., Malamud, B.D., Winter, H., Joly-Laugel, A., 2019. A review of quantification methodologies for multi-hazard interrelationships. Earth-Science Rev. 196, 102881. https://doi.org/https://doi.org/10.1016/j.earscirev.2019.102881

Toonen, W.H.J., Middelkoop, H., Konijnendijk, T.Y.M., Macklin, M.G., Cohen, K.M., 2016. The influence of hydroclimatic variability on flood frequency in the Lower Rhine. Earth Surf.

Process. Landforms 41, 1266-1275. https://doi.org/10.1002/esp.3953

Tootle, G.A., Piechota, T.C., 2006. Relationships between Pacific and Atlantic ocean sea surface temperatures and U.S. streamflow variability. Water Resour. Res. 42. 
https://doi.org/10.1029/2005WR004184

Tootle, G.A., Piechota, T.C., Singh, A., 2005. Coupled oceanic-atmospheric variability and U.S. streamflow. Water Resour. Res. 41. https://doi.org/10.1029/2005WR004381

Tošić, I., Unkašević, M., 2014. Analysis of wet and dry periods in Serbia. Int. J. Climatol. 34, 13571368. https://doi.org/10.1002/joc.3757

Trenberth, K.E., 2011. Changes in precipitation with climate change. Clim. Res. 47, 123-138.

Trenberth, K.E., 1997. The Definition of El Niño. Bull. Am. Meteorol. Soc. 78, 2771-2778.

Trenberth, K.E., Dai, A., van der Schrier, G., Jones, P.D., Barichivich, J., Briffa, K.R., Sheffield, J., 2013. Global warming and changes in drought. Nat. Clim. Chang. 4, 17.

Trenberth, K.E., Fasullo, J.T., 2012. Climate extremes and climate change: The Russian heat wave and other climate extremes of 2010. J. Geophys. Res. Atmos. 117.

https://doi.org/10.1029/2012JD018020

Turner, S.W.D., Hejazi, M., Kim, S.H., Clarke, L., Edmonds, J., 2017. Climate impacts on hydropower and consequences for global electricity supply investment needs. Energy 141, 2081-2090. https://doi.org/https://doi.org/10.1016/j.energy.2017.11.089

Tyler, J.J., Jones, M., Arrowsmith, C., Allott, T., Leng, M.J., 2016. Spatial patterns in the oxygen isotope composition of daily rainfall in the British Isles. Clim. Dyn. 47, 1971-1987. https://doi.org/10.1007/s00382-015-2945-y

Uhlemann, S., Thieken, A.H., Merz, B., 2014. A quality assessment framework for natural hazard event documentation: Application to trans-basin flood reports in Germany. Nat. Hazards Earth Syst. Sci. 14, 189-208. https://doi.org/10.5194/nhess-14-189-2014

Uhlemann, S., Thieken, A.H., Merz, B., 2010. A consistent set of trans-basin floods in Germany between 1952\&ndash;2002. Hydrol. Earth Syst. Sci. 14, 1277-1295. https://doi.org/10.5194/hess-14-1277-2010

Ulbrich, U., Leckebusch, G.C., Pinto, J.G., 2009. Extra-tropical cyclones in the present and future climate: A review. Theor. Appl. Climatol. 96, 117-131. https://doi.org/10.1007/s00704-0080083-8

UNDRR, 2017a. UN Sendai Framework for Disaster Risk Reduction (Terminology).

UNDRR, 2017b. Flood hazard and risk assessment.

UNDRR, 2016. Report of the open-ended intergovernmental expert working group on indicators and terminology relating to disaster risk reduction.

UNDRR, 2015. Sendai Framework for Disaster Risk Reduction 2015-2030.

Van Dijk, A.I.J.M., Beck, H.E., Crosbie, R.S., De Jeu, R.A.M., Liu, Y.Y., Podger, G.M., Timbal, B., Viney, N.R., 2013. The Millennium Drought in southeast Australia (2001-2009): Natural and 
human causes and implications for water resources, ecosystems, economy, and society. Water Resour. Res. 49, 1040-1057. https://doi.org/10.1002/wrcr.20123

Vicente-Serrano, S.M., Beguería, S., López-Moreno, J.I., 2010. A Multiscalar Drought Index Sensitive to Global Warming: The Standardized Precipitation Evapotranspiration Index. J. Clim. 23, 1696-1718. https://doi.org/10.1175/2009JCLI2909.1

Viglione, A., Chirico, G.B., Komma, J., Woods, R., Borga, M., Blöschl, G., 2010. Quantifying space-time dynamics of flood event types. J. Hydrol. 394, 213-229. https://doi.org/10.1016/j.jhydrol.2010.05.041

Visser-Quinn, A., Beevers, L., Collet, L., Formetta, G., Smith, K., Wanders, N., Thober, S., Pan, M., Kumar, R., 2019. Spatio-temporal analysis of compound hydro-hazard extremes across the UK. Adv. Water Resour. 130, 77-90. https://doi.org/https://doi.org/10.1016/j.advwatres.2019.05.019 Wallingford: Centre for Ecology \& Hydrology, 2009. FEH CD-ROM 3.

Wallingford Hydrosolutions, 2016. The Revitalised Flood Hydrograph Model ReFH 2.2: Technical Guidance. Wallingford Wallingford Hydrolsolutions Cent. Ecol. Hydrol.

Wang, H., He, B., Zhang, Y., Huang, L., Chen, Z., Liu, J., 2018. Response of ecosystem productivity to dry/wet conditions indicated by different drought indices. Sci. Total Environ. 612, 347-357. https://doi.org/https://doi.org/10.1016/j.scitotenv.2017.08.212

Wang, J., Kim, H.-M., Chang, E.K.M., 2018. Interannual Modulation of Northern Hemisphere Winter Storm Tracks by the QBO. Geophys. Res. Lett. 45, 2786-2794. https://doi.org/10.1002/2017GL076929

Wang, S., Huang, J., He, Y., Guan, Y., 2014. Combined effects of the Pacific Decadal Oscillation and El Niño-Southern Oscillation on Global Land Dry-Wet Changes. Sci. Rep. 4, 6651.

Wang, Y., Chen, X., Chen, Y., Liu, M., Gao, L., 2015. Flood/drought event identification using an effective indicator based on the correlations between multiple time scales of the Standardized Precipitation Index and river discharge. Theor. Appl. Climatol. 1-10. https://doi.org/10.1007/s00704-015-1699-0

Ward, P.J., Beets, W., Bouwer, L.M., Aerts, J.C.J.H., Renssen, H., 2010. Sensitivity of river discharge to ENSO. Geophys. Res. Lett. 37. https://doi.org/10.1029/2010GL043215

Ward, P.J., Couasnon, A., Eilander, D., Haigh, I.D., Hendry, A., Muis, S., Veldkamp, T.I.E., Winsemius, H.C., Wahl, T., 2018. Dependence between high sea-level and high river discharge increases flood hazard in global deltas and estuaries. Environ. Res. Lett. 13, 84012. https://doi.org/10.1088/1748-9326/aad400

Ward, P.J., Eisner, S., Flörke, M., Dettinger, M.D., Kummu, M., 2014a. Annual flood sensitivities to El Niño-Southern Oscillation at the global scale. Hydrol. Earth Syst. Sci. 18, 47-66. 
https://doi.org/10.5194/hess-18-47-2014

Ward, P.J., Jongman, B., Kummu, M., Dettinger, M.D., Sperna Weiland, F.C., Winsemius, H.C., 2014b. Strong influence of El Niño Southern Oscillation on flood risk around the world. Proc. Natl. Acad. Sci. 111, 15659-15664.

Watkins, S., Whyte, I., 2008. Extreme Flood Events in Upland Catchments in Cumbria since 1600: the evidence of historical records. North West Geogr. 8.

Webster, P.J., Holland, G.J., Curry, J.A., Chang, H.-R., 2005. Changes in Tropical Cyclone Number, Duration, and Intensity in a Warming Environment. Science (80-. ). 309, 1844 LP - 1846. https://doi.org/10.1126/science.1116448

Weinkle, J., Maue, R., Pielke, R., 2012. Historical Global Tropical Cyclone Landfalls. J. Clim. 25, 4729-4735. https://doi.org/10.1175/JCLI-D-11-00719.1

Wells, N., Goddard, S., Hayes, M.J., 2004. A Self-Calibrating Palmer Drought Severity Index. J. Clim. 17, 2335-2351.

Wetterhall, F., Pappenberger, F., He, Y., Freer, J., Cloke, H.L., 2012. Conditioning model output statistics of regional climate model precipitation on circulation patterns. Nonlinear Process.

Geophys. 19, 623-633. https://doi.org/10.5194/npg-19-623-2012

Whittaker, J., 2009. Graphical Models in Applied Multivariate Statistics. Wiley Publishing. Wilby, R., Keenan, R., 2012. Adapting to flood risk under climate change. Prog. Phys. Geogr. 36, $348-378$.

Wilby, R.L., 2008. Constructing Climate Change Scenarios of Urban Heat Island Intensity and Air Quality. Environ. Plan. B Plan. Des. 35, 902-919. https://doi.org/10.1068/b33066t

Wilby, R.L., 1998. Modelling low-frequency rainfall events using airflow indices, weather patterns and frontal frequencies. J. Hydrol. 212-213, 380-392. https://doi.org/https://doi.org/10.1016/S0022-1694(98)00218-2

Wilby, R.L., 1995. Simulation of precipitation by weather pattern and frontal analysis. J. Hydrol. 173, 91-109. https://doi.org/https://doi.org/10.1016/0022-1694(95)02715-2

Wilby, R.L., 1994. Stochastic weather type simulation for regional climate change impact assessment. Water Resour. Res. 30, 3395-3403. https://doi.org/10.1029/94WR01840

Wilby, R.L., 1993. The influence of variable weather patterns on river water quantity and quality regimes. Int. J. Climatol. 13, 447-459. https://doi.org/10.1002/joc.3370130408

Wilby, R.L., Barker, P.A., 2016. Wettest December in the Lake District for over 200 years. Weather 71, 76. https://doi.org/10.1002/wea.2711

Wilby, R.L., Dalgleish, H.Y., Foster, I.D.L., 1997. The impact of weather patterns on historic and contemporary catchment sediment yields. Earth Surf. Process. Landforms 22, 353-363. 
Wilby, R.L., Jones, P.D., Lister, D.H., 2011. Decadal variations in the nocturnal heat island of London. Weather 66, 59-64. https://doi.org/10.1002/wea.679

Wilby, R.L., Quinn, N.W., 2013. Reconstructing multi-decadal variations in fluvial flood risk using atmospheric circulation patterns. J. Hydrol. 487, 109-121.

https://doi.org/10.1016/j.jhydrol.2013.02.038

Wilby, R.L., Wigley, T.M.L., 1997. Downscaling general circulation model output: a review of methods and limitations. Prog. Phys. Geogr. Earth Environ. 21, 530-548. https://doi.org/10.1177/030913339702100403

Wild, S., Befort, D., Leckebusch, G.C., 2015. Was the Extreme Storm Season in Winter 2013/14

Over the North Atlantic and the United Kingdom Triggered by Changes in the West Pacific Warm Pool?, [in "Explaining Extremes of 2014 from a Climate Perspective"]. Bull. Amer. Meteor. Soc. 96.

Winsemius, H.C., Aerts, J.C.J.H., van Beek, L.P.H., Bierkens, M.F.P., Bouwman, A., Jongman, B., Kwadijk, J.C.J., Ligtvoet, W., Lucas, P.L., van Vuuren, D.P., Ward, P.J., 2016. Global drivers of future river flood risk. Nat. Clim. Chang. 6, 381-385.

Woollings, T., Barriopedro, D., Methven, J., Son, S.-W., Martius, O., Harvey, B., Sillmann, J., Lupo, A.R., Seneviratne, S., 2018. Blocking and its Response to Climate Change. Curr. Clim. Chang. Reports. https://doi.org/10.1007/s40641-018-0108-z

Woollings, T., Harvey, B., Masato, G., 2014. Arctic warming, atmospheric blocking and cold European winters in CMIP5 models. Environ. Res. Lett. 9, 14002.

Wyncoll, D., Gouldby, B., 2013. Integrating a multivariate extreme value method within a system flood risk analysis model. J. Flood Risk Manag.

Xie, W., Xiong, W., Pan, J., Ali, T., Cui, Q., Guan, D., Meng, J., Mueller, N.D., Lin, E., Davis, S.J., 2018. Decreases in global beer supply due to extreme drought and heat. Nat. Plants 4, 964-973. https://doi.org/10.1038/s41477-018-0263-1

Xu, H., Corte-Real, J., Qian, B., 2007. Developing daily precipitation scenarios for climate change impact studies in the Guadiana and the Tejo basins. Hydrol. Earth Syst. Sci. 11, 1161-1173. https://doi.org/10.5194/hess-11-1161-2007

Yan, D.H., Wu, D., Huang, R., Wang, L.N., Yang, G.Y., 2013. Drought evolution characteristics and precipitation intensity changes during alternating dry-wet changes in the Huang-Huai-Hai River basin. Hydrol. Earth Syst. Sci. 17, 2859-2871. https://doi.org/10.5194/hess-17-2859-2013 Yoon, J.-H., Wang, S.-Y.S., Lo, M.-H., Wu, W.-Y., 2018. Concurrent increases in wet and dry extremes projected in Texas and combined effects on groundwater. Environ. Res. Lett. 13, 54002. https://doi.org/10.1088/1748-9326/aab96b 
Zampieri, M., Ceglar, A., Dentener, F., Toreti, A., 2017. Wheat yield loss attributable to heat waves, drought and water excess at the global, national and subnational scales. Environ. Res. Lett. 12, 64008. https://doi.org/10.1088/1748-9326/aa723b

Zappa, G., Shaffrey, L.C., Hodges, K.I., Sansom, P.G., Stephenson, D.B., 2013. A multimodel assessment of future projections of north atlantic and european extratropical cyclones in the CMIP5 climate models. J. Clim. 26, 5846-5862. https://doi.org/10.1175/JCLI-D-12-00573.1

Zhang, F., Cai, X., Thornes, J.E., 2014. Birmingham's air and surface urban heat islands associated with Lamb weather types and cloudless anticyclonic conditions. Prog. Phys. Geogr. Earth

Environ. 38, 431-447. https://doi.org/10.1177/0309133314538725

Zhang, Q., Gu, X., Singh, V.P., Kong, D., Chen, X., 2015. Spatiotemporal behavior of floods and droughts and their impacts on agriculture in China. Glob. Planet. Change 131, 63-72. https://doi.org/https://doi.org/10.1016/j.gloplacha.2015.05.007

Zhang, Q., Zhang, W., Chen, Y.D., Jiang, T., 2011. Flood, drought and typhoon disasters during the last half-century in the Guangdong province, China. Nat. Hazards 57, 267-278.

$$
\text { https://doi.org/10.1007/s11069-010-9611-9 }
$$

Zhong, S., Clark, M., Hou, X.-Y., Zang, Y.-L., FitzGerald, G., 2013. 2010-2011 Queensland floods: Using Haddon's Matrix to define and categorise public safety strategies. Emerg. Med. Australas. 25, 345-352. https://doi.org/10.1111/1742-6723.12097

Zhou, Y., Liu, Y., Wu, W., Li, N., 2015. Integrated risk assessment of multi-hazards in China. Nat. Hazards 78, 257-280. https://doi.org/10.1007/s11069-015-1713-y

Zscheischler, J., Seneviratne, S.I., 2017. Dependence of drivers affects risks associated with compound events. Sci. Adv. 3, e1700263. https://doi.org/10.1126/sciadv.1700263

Zscheischler, J., Westra, S., van den Hurk, B.J.J.M., Seneviratne, S.I., Ward, P.J., Pitman, A., AghaKouchak, A., Bresch, D.N., Leonard, M., Wahl, T., Zhang, X., 2018. Future climate risk from compound events. Nat. Clim. Chang. 8, 469-477. https://doi.org/10.1038/s41558-018- 